\title{
Methods of Practice and Guidelines for Using Survey-Grade Global Navigation Satellite Systems (GNSS) to Establish Vertical Datum in the United States Geological Survey
}

Chapter 1 of

Section D, Field Survey Methods

Book 11, Collection and Delineation of Spatial Data

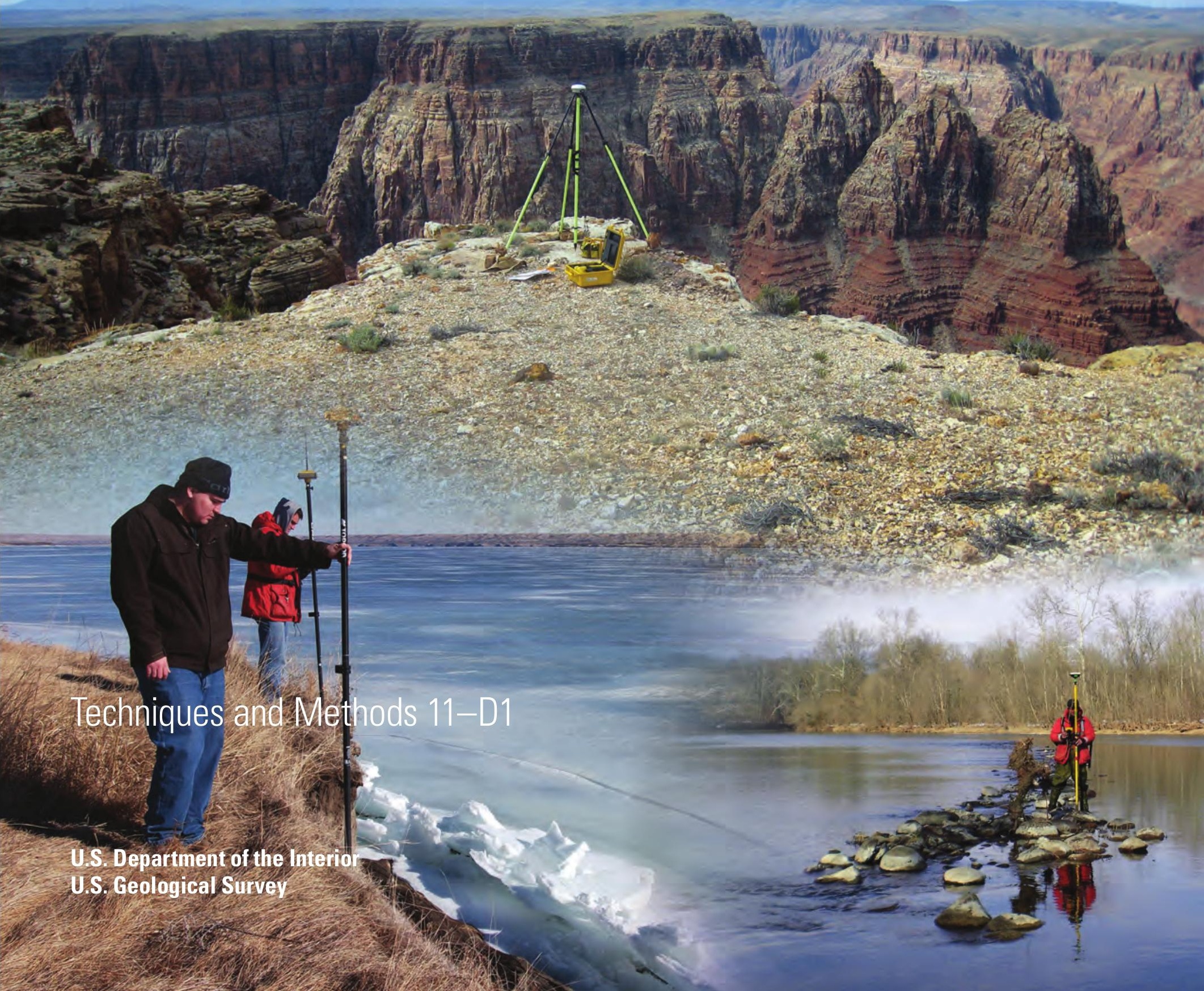


Front cover. Background: Grand Canyon looking down on the Little Colorado River, on the Navajo Nation. Photograph by Jeffrey R. Kennedy, 2009, USGS.

Lower right: Paul Kinzel (USGS) measuring water-surface elevation along a rock-wing dam in the South Fork Shenandoah River near Island Ford, Virginia. Photograph by Jennifer L. Krstolic, 2010, USGS.

Lower left: Western shore of Lake Sharpe near Lower Brule, South Dakota. Vaughn Big Eagle (Lower Brule Sioux Tribe) is in the foreground, and Ryan F. Thompson (USGS) is in the background. Photograph by Kathy Neitzert, USGS. 


\section{Methods of Practice and Guidelines for Using Survey-Grade Global Navigation Satellite Systems (GNSS) to Establish Vertical Datum in the United States Geological Survey}

By Paul H. Rydlund, Jr., and Brenda K. Densmore

Chapter 1 of

Section D, Field Survey Methods

Book 11, Collection and Delineation of Spatial Data

Techniques and Methods 11-D1 


\section{U.S. Department of the Interior \\ KEN SALAZAR, Secretary \\ U.S. Geological Survey \\ Marcia K. McNutt, Director}

\section{U.S. Geological Survey, Reston, Virginia: 2012}

For more information on the USGS - the Federal source for science about the Earth, its natural and living resources, natural hazards, and the environment, visit http://www.usgs.gov or call 1-888-ASK-USGS.

For an overview of USGS information products, including maps, imagery, and publications, visit http://www.usgs.gov/pubprod

To order this and other USGS information products, visit http://store.usgs.gov

Any use of trade, firm, or product names is for descriptive purposes only and does not imply endorsement by the U.S. Government.

Although this information product, for the most part, is in the public domain, it also may contain copyrighted materials as noted in the text. Permission to reproduce copyrighted items must be secured from the copyright owner.

Suggested citation:

Rydlund, P.H., Jr., and Densmore, B.K., 2012, Methods of practice and guidelines for using survey-grade global navigation satellite systems (GNSS) to establish vertical datum in the United States Geological Survey: U.S. Geological Survey Techniques and Methods, book 11, chap. D1, 102 p. with appendixes. 


\section{Preface}

This series of manuals on techniques and methods (TM) describes approved scientific and data collection procedures and standard methods for planning and executing studies and laboratory analyses. The material is grouped under primary subject headings called "books" and further subdivided into sections and chapters. This manual is Chapter 1 of Section D-Field Survey Methods, Book 11—Collection and Delineation of Spatial Data.

The unit of publication, the chapter, is limited to a narrow field of subject matter. These publications are subject to revision because of experience in use or because of advancement in knowledge, techniques, or equipment, and this format permits flexibility in revision and publication as the need arises. Chapter D1 of book 11 (TM 11-D1) deals with vertical datum establishment using survey-grade Global Navigation Satellite Systems (GNSS).

This edition of "Methods of Practice and Guidelines for Using Survey-Grade Global Navigation Satellite Systems (GNSS) to Establish Vertical Datum in the United States Geological Survey" is published on the World Wide Web at http://pubs.usgs.gov/tm/tm11D1/ and is for sale by the U.S. Geological Survey, Science Information Delivery, Box 25286, Federal Center, Denver, Colorado, 80225.

\section{Acknowledgments}

The authors would like to thank Mr. Dave Doyle, Mr. William Henning, and Mr. Mark Schenewerk from the National Geodetic Survey for technical guidance contained within. Published manuals from the National Geodetic Survey have been used as the foundation for this technical manual with modifications to adequately suit data collection and science needs within the U.S. Geological Survey. The authors would also like to further thank Mr. Tom Bryant from Seiler Instrument, Inc., for lengthy conversations regarding practice and quality of Global Navigation Satellite System (GNSS) surveys. 



\section{Contents}

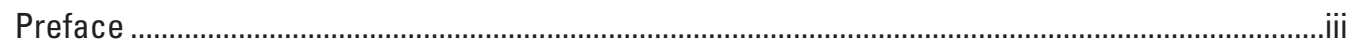

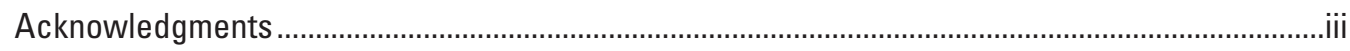

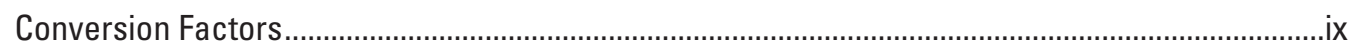

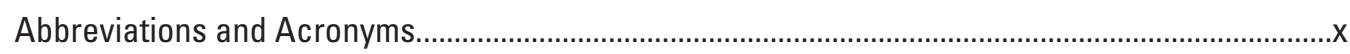

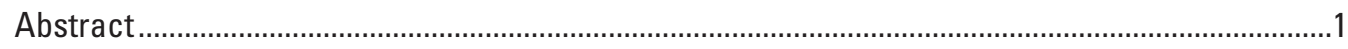

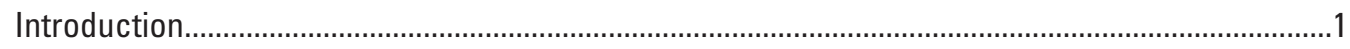

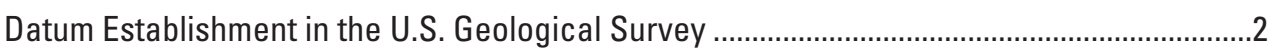

Evolution of Global Navigation Satellite Systems ..................................................................

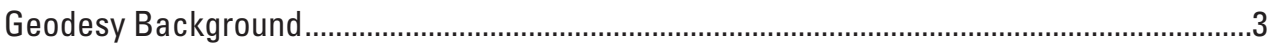

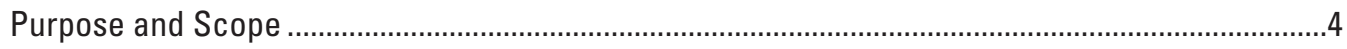

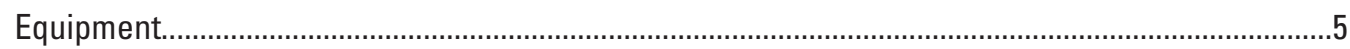

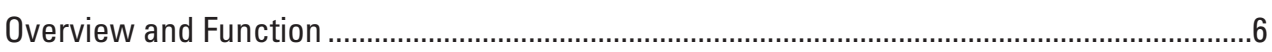

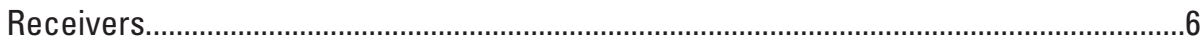

Antenna (Receiver and Radio) and Radio ...................................................................

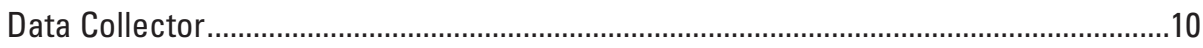

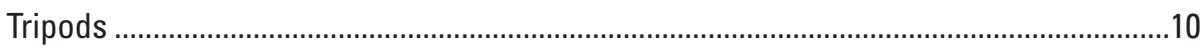

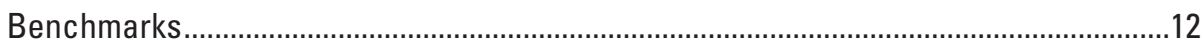

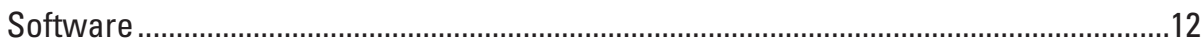

GNSS Equipment Summary and Checklist ................................................................16

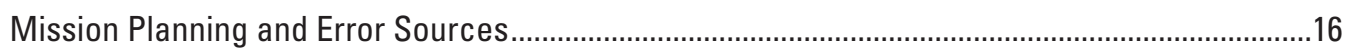

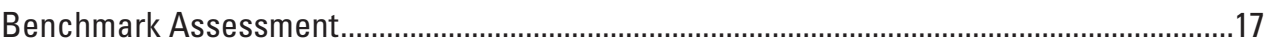

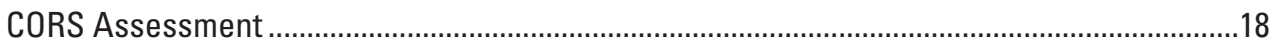

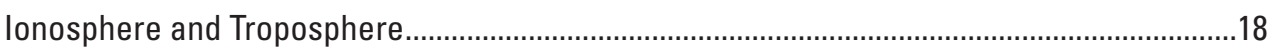

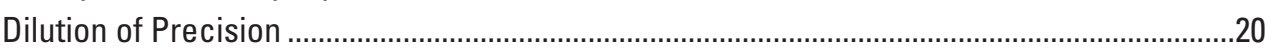

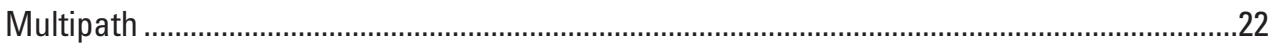

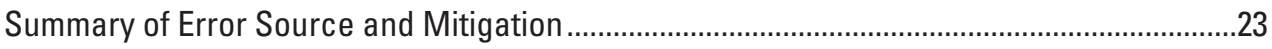

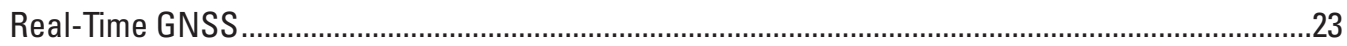

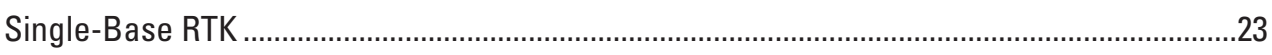

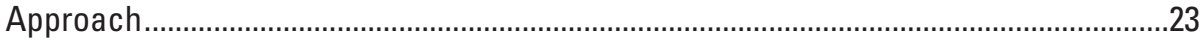

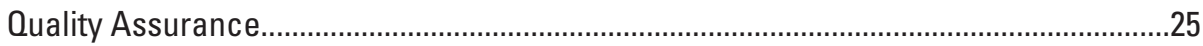

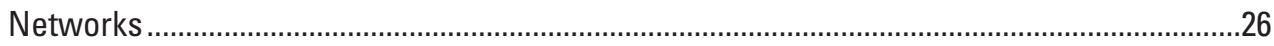

Approach .

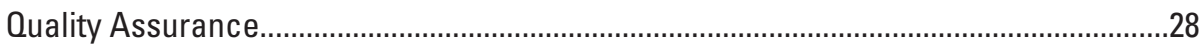

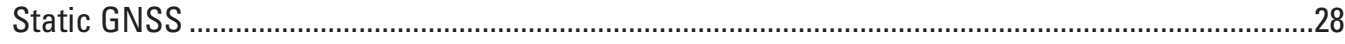

Single Base: Online Positioning User Service (OPUS) ………..............................................2

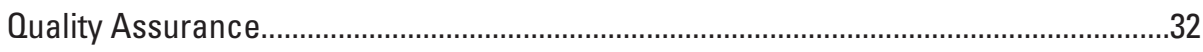

Additions and Advancements ...................................................................................

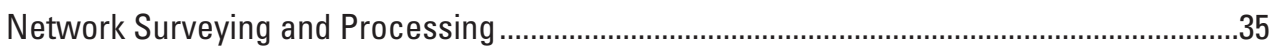

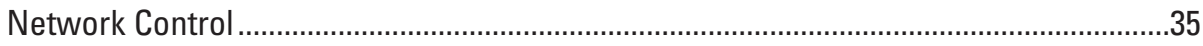

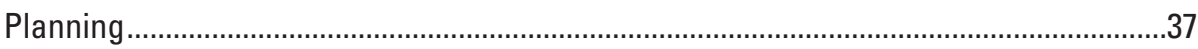

Processing and Adjustment................................................................................. 41

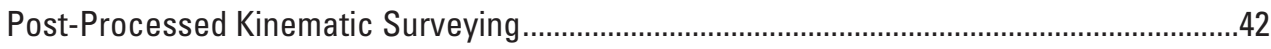


GNSS Ouality
Level I Survey
Level II Survey
Level III Survey
Level IV Survey.
Level Quality Alternatives with Real-Time Positioning
Uncertainty Analysis
Metadata
Database Storage

\section{Figures}

1. Illustration showing relation between the ellipsoid, geoid, and orthometric heights ......4

2. Photograph showing tribrachs used with tripods to attach Global Navigation Satellite System receivers

3. Photographs showing various types of Global Navigation Satellite System

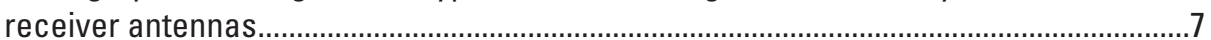

4. Photographs showing traditional real-time equipment profile ........................................8

5. Photograph showing "rubber duck" antenna used for radio communication....................9

6. Photographs showing Real-Time Kinematic bridge used to facilitate multiple survey-grade receivers within a Real-Time Network for areas void of cellular coverage

7. Photographs showing data collectors used for Global Navigation Satellite System positioning 
8. Photographs showing fixed-height and adjustable fixed-height center pole tripod and bipod used for Global Navigation Satellite System positioning ................................11

9. Photographs showing circular level vial adjustment on fixed-height center poles........13

10. Photographs showing examples of monumented and non-monumented benchmarks and anchoring construction used for Global Navigation Satellite System observations

11. Map showing depth of frozen ground for the lower 48 United States.

12. Map showing density and proximity of Continually Operating Reference Station (managed by the National Geodetic Survey in the Midwest

13. Graph showing progression and forecast of ionospheric disturbance because of strong magnetic fields associated with sun spots.

14. Illustration showing elevation mask angle in relation to Global Navigation Satellite System signals traveling through the ionosphere and troposphere . .20

15. Illustrations showing horizontal and vertical relation to quality dilution of precision ....21

16. Illustration showing multipath development from illustrated objects.

17. Illustration showing example approach using single-base Real-Time Kinematic as a level

18. Illustration showing Real-Time Network positioning with a 4-point localization to known benchmarks

19. Illustration showing Real-Time Network positioning using the interpolation methodology of a virtual base station

20. Screen capture showing modified output of a position generated from the Online Position User Service-Rapid Static.....

21. Illustration showing simple network design with resurveys and baselines . .38

22. Screen capture showing example results as displayed in the data collector of a localization with Global Navigation Satellite System

23. Diagram showing decision tree for a level quality Global Navigation Satellite System survey approach

\section{Appendix Figures}

3-1. Map showing background for a high-water mark survey along the Salt River near Brashear, Missouri

3-2. Map showing background for streamgage datum establishment ...................................76

3-3. Map showing background for a groundwater well field survey .....................................78

4-1. Map showing recovered and established benchmarks for the topographic and bathymetric survey at Lewis and Clark Lake....

4-2. Figures and graphs showing time series and data availability plots downloaded from the National Geodetic Survey Continuously Operating Reference Station website ..........82

4-3. Map showing objective points established at locations 1013 and 1014 using a static network survey that includes controlling National Geodetic Survey benchmarks

4-4. Screen capture showing adjustment software depicting Global Navigation Satellite System receiver data file information and location; including antenna type and height, and observation times.

4-5. Screen capture showing network adjustment output representing initial processing steps and a minimally constrained adjustment of the Missouri River network

4-6. Screen capture showing adjustment software package depicting processed and deselected baselines in the Missouri River network. 
4-7. Screen capture showing adjustment software depicting coordinates entered for National Geodetic Survey benchmarks and one benchmark held as fixed for the minimally constrained adjustment.

4-8. Screen capture showing adjustment software package depicting results of the minimally constrained network adjustment...

4-9. Screen capture showing adjustment software depicting control tie information and quality-assurance test from the minimally constrained Missouri River network adjustment.

4-10. Screen capture showing adjustment software depicting coordinates for the newly established benchmarks resulting from the Missouri River final network adjustment......96

4-11. Screen capture showing an editable mock-up survey datasheet within the Online Position User Service Projects web-based utility identifying an objective point established at a local streamgage.

4-12. Screen capture showing an editable mock-up survey datasheet within the Online Position User Service Projects web-based utility identifying an objective point established as a ground control point for a terrestrial laser scan

4-13. Screen capture showing static survey network developed within Online Position Users Service projects web-based utility

4-14. Screen capture showing Online Position User Service projects session processing of a network of Global Navigation Satellite System observations

4-15. Screen capture showing adjustment performed within the Online Position User Service Projects web-based utility

\section{Tables}

1. Primary navigation satellite systems

2. Residual differences between the Geoid 2009 hybrid model and known separation distance as determined from Global Positioning System-derived ellipsoid heights and leveled North American Vertical Datum 1988 orthometric heights at benchmarks within the conterminous United States.

3. Global Navigation Satellite System equipment checklist and quality-assurance recommendations.

4. Global Navigation Satellite System error source summary and mitigation

5. User benefits and drawbacks to Real-Time Network Global Navigation Satellite Systems.

6. Example of Real-Time Network reference station positioning change in Missouri after adoption into the National Geodetic Survey Continually Operating Reference Station network

7. Real-time Global Navigation Satellite System quality assurances for single-base

Real-Time Kinematic and a Real-Time Network

8. NAD 83 realizations, marks used in each realization, and comments about each adjustment

9. Accuracy standard for leveled, triangulated, and traversed benchmarks

10. Accuracy standard for Global Positioning System-derived benchmarks

11. Level-quality descriptions for Global Navigation Satellite System positioning in the U.S. Geological Survey.

12. An example of summarized observations and quality criteria upheld for a Level II real-time survey using a Real-Time Network

13. Uncertainty of high-water marks for coastal storm surge and upland rivers. 


\section{Appendix Tables}

3-1. Assessment of benchmarks for perpetuating datum to U.S. Geological Survey streamgages.

4-1. Benchmark quality indicators from Online Position Users Service results for datum establishment used for a river survey.

4-2. Static network survey uncertainty analysis using Online Position User Service projects results

\section{Conversion Factors and Datums}

Inch/Pound to SI

\begin{tabular}{lcl}
\hline \multicolumn{1}{c}{ Multiply } & By & \multicolumn{1}{c}{ To obtain } \\
\hline inch (in.) & Length & \\
inch (in.) & 2.54 & centimeter $(\mathrm{cm})$ \\
foot (ft) & 25.4 & millimeter $(\mathrm{mm})$ \\
mile (mi) & 0.3048 & meter $(\mathrm{m})$ \\
mile, nautical (nmi) & 1.609 & kilometer $(\mathrm{km})$ \\
yard (yd) & 1.852 & kilometer $(\mathrm{km})$ \\
\hline & 0.9144 & meter $(\mathrm{m})$ \\
\hline acre & Area & \\
acre & 4,047 & square meter $\left(\mathrm{m}^{2}\right)$ \\
square foot $\left(\mathrm{ft}^{2}\right)$ & 0.004047 & square kilometer $\left(\mathrm{km}^{2}\right)$ \\
square foot $\left(\mathrm{ft}^{2}\right)$ & 929.0 & square centimeter $\left(\mathrm{cm}^{2}\right)$ \\
square inch $\left(\mathrm{in}^{2}\right)$ & 0.09290 & square meter $\left(\mathrm{m}^{2}\right)$ \\
square mile $\left(\mathrm{mi}^{2}\right)$ & 6.452 & square centimeter $\left(\mathrm{cm}^{2}\right)$ \\
\hline
\end{tabular}

Temperature in degrees Celsius $\left({ }^{\circ} \mathrm{C}\right)$ may be converted to degrees Fahrenheit $\left({ }^{\circ} \mathrm{F}\right)$ as follows: ${ }^{\circ} \mathrm{F}=\left(1.8 \times^{\circ} \mathrm{C}\right)+32$

Temperature in degrees Fahrenheit $\left({ }^{\circ} \mathrm{F}\right)$ may be converted to degrees Celsius $\left({ }^{\circ} \mathrm{C}\right)$ as follows: ${ }^{\circ} \mathrm{C}=\left({ }^{\circ} \mathrm{F}-32\right) / 1.8$

Vertical coordinate information is referenced to the North American Vertical Datum of 1988 (NAVD 88).

Horizontal coordinate information is referenced to the North American Datum of 1983 (NAD 83).

Altitude, as used in this manual, refers to distance above the vertical datum.

Global Positioning Systems (GPS) is used in this manual to define the U.S. space-based Global Navigation Satellite System (GNSS). 


\section{Abbreviations and Acronyms}

$3 \mathrm{D}$

APC

ARP

C/A Code

CAD

CBN

$\mathrm{CMR}+$

CONUS

CORS

DOT

ECEF

FBN

FGCC

FOC

GDOP

GEOID 2009

GIS

GLONASS

GNSS

GPS

GRAV-D

GRS 80

GWSI

HDOP

IERS

IGS

ITRF

ITRS

LAN

$\mathrm{L}_{1}$

$L_{1} \mathrm{C}$

$\mathrm{L}_{2}$

$\mathrm{L}_{2} \mathrm{C}$

$L_{5}$
Three Dimensional

Antenna Phase Center

Antenna Reference Point

Coarse Acquisition Code

Computer Automated Drafting

Cooperative Based Network

Compact Measurement Record

Conterminous United States

Continuously Operating Reference Station

Department of Transportation

Earth Centered Earth Fixed

Federal Based Network

Federal Geodetic Control Committee

Fully Operational Capability

Geometric Dilution of Precision

Hybrid Geoid Model of 2009

Geographic Information System

GLObal NAvigation Satellite System-Russian

Global Navigation Satellite System-Universal

U.S. Space-based Global Positioning System

Gravity for the Redefinition of the American Vertical Datum

Geodetic Reference System 1980

Ground-Water Site-Inventory

Horizontal Dilution of Precision

International Earth Rotation and Reference System Service

International GNSS Service

International Terrestrial Reference Frame

International Terrestrial Reference System

Local Area Network

GNSS L band carrier wave at $1575.42 \mathrm{MHz}$

GNSS L band carrier wave at $1575.42 \mathrm{MHz}$ with optimized signal design

GNSS L band carrier wave at $1227.60 \mathrm{MHz}$

GNSS L band carrier wave at $1227.60 \mathrm{MHz}$ with optimized signal design

GPS L band carrier wave at $1176.45 \mathrm{MHz}$ 


\begin{tabular}{|c|c|}
\hline LiDAR & Light Detection and Ranging \\
\hline LMR 200 & Standard Type Coaxial Cable \\
\hline $\mathrm{MHz}$ & Megahertz (1 million cycles per second) \\
\hline NAD 83 & North American Datum 1983 \\
\hline NANU & Notice Advisory to NAVSTAR Users \\
\hline NAVD 88 & North American Vertical Datum 1988 \\
\hline NAVSTAR & Navigation Satellite Timing and Ranging \\
\hline NDEP & National Digital Elevation Program \\
\hline NGS & National Geodetic Survey \\
\hline NGS IDB & National Geodetic Survey Integrated Database \\
\hline NGVD 29 & National Geodetic Vertical Datum of 1929 \\
\hline NMD & National Mapping Division \\
\hline NOAA & National Oceanic and Atmospheric Administration \\
\hline NSRS & National Spatial Reference System \\
\hline NSSDA & National Standard for Spatial Data Accuracy \\
\hline NWS & National Weather Service \\
\hline OPUS & Online Position Users Service \\
\hline OPUS-DB & Online Position Users Service Database \\
\hline OPUS GNSS & Online Position Users Service-GNSS \\
\hline OPUS LOCUS & Online Position Users Service_-Leveling Online Computing Service \\
\hline OPUS-RS & Online Position Users Service-Rapid Static \\
\hline OPUS-S & Online Position Users Service-Static \\
\hline P Code & Precision Code \\
\hline PAGES & Program for the Adjustment of GPS Ephemerides \\
\hline PDOP & Position Dilution of Precision \\
\hline PID & Permanent Identifier \\
\hline PPM & Part(s) per Million \\
\hline PPP & Precise Point Positioning \\
\hline PRVD 02 & Puerto Rico Vertical Datum of 2002 \\
\hline QA & Quality Assurance \\
\hline RG 58 & Radio Guide Standard Type Coaxial Cable \\
\hline RINEX & Receiver Independent Exchange format conversion \\
\hline RMS & Root Mean Square \\
\hline RSGPS & Rapid Static Global Positioning System \\
\hline RT & Real-Tme Positioning \\
\hline
\end{tabular}




$\begin{array}{ll}\text { RTCM } & \text { Radio Technical Commission for Maritime Services } \\ \text { RTK } & \text { Real-Time Kinematic } \\ \text { RTN } & \text { Real-Time Network(s) } \\ \text { SBAS } & \text { Satellite Based Augmentation System } \\ \text { SNR } & \text { Signal-To-Noise Ratio } \\ \text { SWPC } & \text { Space Weather Prediction Center } \\ \text { TDOP } & \text { Time Dilution of Precision } \\ \text { TEC } & \text { Total Electron Content } \\ \text { USACE } & \text { U.S. Army Corps of Engineers } \\ \text { USCGS } & \text { U.S. Coastal and Geodetic Survey } \\ \text { USGG 2009 } & \text { U.S. Gravimetric Geoid of 2009 } \\ \text { USGS } & \text { U.S. Geological Survey } \\ \text { USNO } & \text { U.S. Naval Observatory } \\ \text { UTM } & \text { Universal Transverse Mercator } \\ \text { VDOP } & \text { Vertical Dilution of Precision } \\ \text { WAAS } & \text { Wide Area Augmentation System } \\ \text { WGS 84 } & \text { World Geodetic System 1984 }\end{array}$




\title{
Methods of Practice and Guidelines for Using Survey-Grade Global Navigation Satellite Systems (GNSS) to Establish Vertical Datum in the United States Geological Survey
}

\author{
By Paul H. Rydlund, Jr., and Brenda K. Densmore
}

\begin{abstract}
Geodetic surveys have evolved through the years to the use of survey-grade (centimeter level) global positioning to perpetuate and post-process vertical datum. The U.S. Geological Survey (USGS) uses Global Navigation Satellite Systems (GNSS) technology to monitor natural hazards, ensure geospatial control for climate and land use change, and gather data necessary for investigative studies related to water, the environment, energy, and ecosystems. Vertical datum is fundamental to a variety of these integrated earth sciences.

Essentially GNSS surveys provide a three-dimensional position $\mathrm{x}, \mathrm{y}$, and $\mathrm{z}$ as a function of the North American Datum of 1983 ellipsoid and the most current hybrid geoid model. A GNSS survey may be approached with postprocessed positioning for static observations related to a single point or network, or involve real-time corrections to provide positioning "on-the-fly." Field equipment required to facilitate GNSS surveys range from a single receiver, with a power source for static positioning, to an additional receiver or network communicated by radio or cellular for real-time positioning. A real-time approach in its most common form may be described as a roving receiver augmented by a single-base station receiver, known as a single-base real-time (RT) survey. More efficient real-time methods involving a Real-Time Network (RTN) permit the use of only one roving receiver that is augmented to a network of fixed receivers commonly known as Continually Operating Reference Stations (CORS). A post-processed approach in its most common form involves static data collection at a single point. Data are most commonly post-processed through a universally accepted utility maintained by the National Geodetic Survey (NGS), known as the Online Position User Service (OPUS). More complex post-processed methods involve static observations among a network of additional receivers collecting static data at known benchmarks. Both classifications provide users flexibility regarding efficiency and quality of data collection.

Quality assurance of survey-grade global positioning is often overlooked or not understood and perceived
\end{abstract}

uncertainties can be misleading. GNSS users can benefit from a blueprint of data collection standards used to ensure consistency among USGS mission areas. A classification of GNSS survey qualities provide the user with the ability to choose from the highest quality survey used to establish objective points with low uncertainties, identified as a Level I, to a GNSS survey for general topographic control without quality assurance, identified as a Level IV. A Level I survey is strictly limited to post-processed methods, whereas Level II, Level III, and Level IV surveys integrate variations of a RT approach. Among these classifications, techniques involving blunder checks and redundancy are important, and planning that involves the assessment of the overall satellite configuration, as well as terrestrial and space weather, are necessary to ensure an efficient and quality campaign. Although quality indicators and uncertainties are identified in post-processed methods using CORS, the accuracy of a GNSS survey is most effectively expressed as a comparison to a local benchmark that has a high degree of confidence. Real-time and post-processed methods should incorporate these "trusted" benchmarks as a check during any campaign.

Global positioning surveys are expected to change rapidly in the future. The expansion of continuously operating reference stations, combined with newly available satellite signals and enhancements to the conterminous geoid, are all sufficient indicators for substantial growth in real-time positioning and quality thereof.

\section{Introduction}

Aside from geodetic surveying, Global Navigation Satellite System (GNSS) positioning has become a well-known technology used heavily in many scientific studies, in addition to real estate, transportation, agriculture, and recreational industries. As science and technology continue to increase demands on global positioning, additional satellites will be placed in orbit, and modernization efforts will continue to provide stronger civilian frequencies to increase quality and efficiency. 


\section{Datum Establishment in the U.S. Geological Survey}

Historically, the U.S. Geological Survey (USGS) has used geodetic leveling to establish elevations that were used as the framework in the development of topographic maps (U.S. Geological Survey, 1966). Typically, the USGS followed third-order quality-control standards (issued by the Bureau of Budget in 1958) when establishing elevation, except in cases where a greater detail of mapping was required (U.S. Geological Survey, 1966). These elevations supplemented the existing network of first- and second-order quality of elevation control established by the U.S. Coastal and Geodetic Survey (USCGS). A lesser degree of accuracy than the third-order standard was documented by Kennedy (1990), who stated that USGS gaging stations are tied to the National Geodetic Vertical Datum of 1929 (NGVD 29) by "ordinary levels." Used in this context, an "ordinary level" may be classified as fourthorder accuracy, which differs from a third-order accuracy by omitting special equipment and meticulous procedures designed to minimize systematic errors (Kennedy, 1990).

Kennedy defined the "ordinary level" approach essentially as a third-order accuracy, keeping errors smaller than $0.05 \sqrt{M}$ feet (ft), (where $M$ is the total distance run, out and back, in miles), but using ordinary equipment. Preserving techniques outlined in the "Topographic Instructions of the United States-Leveling” (U.S. Geological Survey, 1966), Kennedy limited sight lengths to $300 \mathrm{ft}$ and required level rods to be read within $0.01 \mathrm{ft}$ during geodetic leveling. Kennedy identified the first indication of an accuracy requirement in perpetuating a datum to a streamgage $(0.05 \sqrt{M} \mathrm{ft})$, including an exception for hilly or rough terrain where allowable closure errors were kept under $0.10 \sqrt{M} \mathrm{ft}$.

Moving forward from Kennedy (1990), the evolution of technology, particularly GNSS, can satisfy, if not exceed, these expectations in perpetuating a datum from an existing network of benchmarks. The history of global positioning in the USGS originates from the spring of 1986 when the National Mapping Division (NMD) and Geologic Division of the USGS invested in early dual-frequency receivers that had the ability to track 4 of the 6 satellites in orbit for crustal motion and subsidence studies (D. Benson, U.S. Geological Survey, written comm., 2010). As global positioning technology advanced in 1989, the USGS began using receivers that stored data internally (as opposed to external cassette tapes) and were much more portable. In late 1992, NMD purchased dual-frequency receivers that were used during the next 13 years (D. Benson, U.S. Geological Survey, written commun., 2010). As the constellation expanded to 24 available satellites, observation time for data collection was reduced, and these receivers were successful in providing orthophoto control sufficient for mapping and other projects, such as the monitoring of earthquakes.

As interagency efforts and cooperative interests continue to increase within the scientific community, and the use of survey-grade global positioning is growing as an integral part of field data collection in the USGS, documentation is needed to provide guidance regarding the proper use and expression of data uncertainty acquired by GNSS. A sufficient example is the current USGS streamgage network, consisting of approximately 7,500 streamgages (U.S. Geological Survey, National Streamflow Information Program, 2006). Data from the streamgage network are used in many capacities, such as flood control, navigation, transportation infrastructure, flood forecasting, wastewater treatment, hydro-electric power, recreation, and other water-supply and resource management applications vital to human supply and health. Consequently, this network is one of the largest sources of public and cooperative interest (funded in partnership with Federal, State, and local agencies), which highlights the need for perpetuated datum in terms of consistency, reliability, and accuracy. Other survey-grade global positioning applications and requirements specific to the USGS arise from field data collection that supports multi-disciplinary science among the USGS mission areas (http://www.usgs.gov/start_with_science).

\section{Evolution of Global Navigation Satellite Systems}

The original U.S. space-based Global Positioning System (GPS) has greatly evolved after a 24 operational satellite constellation was declared fully operational in 1995, identified as the Navigation Satellite Timing and Ranging (NAVSTAR) system (GlobalSecurity.org, 2010). Upon completion of the fully functional constellation, the availability of selective civilian-use signals was approved as part of a comprehensive national policy by former president Bill Clinton in 1996 (Office of Science and Technology Policy, 1996). As outlined by the 1996 policy, civilian selective availability (a technique employed to degrade GPS signal for national security reasons) was then discontinued in May of the year 2000, thereby benefiting worldwide safety and scientific commercial interests by elimination of the degraded signal. Removal of the degraded signal provided substantial improvement in autonomous global positioning. In 2006, global positioning technology started to evolve to a state of global geo-spatial positioning, known as Global Navigation Satellite Systems (GNSS). GNSS essentially combines globally-functional satellite constellations with global and regional ground-based reference stations (at accurately surveyed locations) to enhance and broaden positioning. The primary navigation satellite systems, composed of GPS, the Russian GLObal NAvigation Satellite System (GLONASS), the European Galileo, and the Chinese Compass are further defined in table 1 (Federal Space Agency, 2011). These navigation satellite systems are augmented with globaland regional-based reference stations that maintain geostationary satellites as part of the Satellite Based Augmentation System (SBAS). One of the most common systems is known as the Wide Area Augmentation System (WAAS), developed by the Federal Aviation Administration for flight navigation. Other common commercial subscription-based GNSS augmentation systems are proprietary. 
Table 1. Primary navigation satellite systems.

[GPS, Global Positioning System; $\geq$, greater than or equal to; GLONASS, GLObal NAvigation Satellite System]

\begin{tabular}{llcl}
\hline Constellation & \multicolumn{1}{c}{ Owner } & $\begin{array}{c}\text { Number of } \\
\text { satellites* }\end{array}$ & \multicolumn{1}{c}{ Year 2010 status } \\
\hline GPS & United States & $\geq 24$ & Operational. \\
GLONASS & Russia & $\geq 24$ & $\begin{array}{l}\text { Operational with restric- } \\
\text { tions. }\end{array}$ \\
Galileo & Europe & $\geq 27$ & $\begin{array}{c}\text { In preparation, operational } \\
\text { expected 2013. }\end{array}$ \\
Compass & China & 35 & $\begin{array}{c}\text { In preparation, operational } \\
\text { expected 2013. }\end{array}$ \\
\hline
\end{tabular}

\footnotetext{
"Number does not include satellites that may be on reserve for backup or more recent additions as part of modernization efforts. Statistics provided in year 2010 (Federal Space Agency, 2011).
}

\section{Geodesy Background}

The science of measuring the size and shape of the Earth, and its relation to precise locations on the Earth's surface is needed to relate and further define applications of global positioning. To define points on the Earth's surface, a spheroidal reference surface is needed in which to perform geodetic computations. The surface of the earth is most effectively represented by an ellipsoid, a mathematically-defined surface flattened slightly at the poles, and bulging somewhat at the equator (Burkard, 1985). The most current ellipsoid reference surface is identified by World Geodetic System 1984 (WGS 84) and is the standard used by the Department of Defense for GPS positioning, mapping, timing, and navigation. In addition to the WGS 84 ellipsoid, it is important to define the associated reference system, which reflects the monitoring of all fixed station coordinates on the Earth as they change within a particular epoch of time because of continental drift (Henning, 2010). The International Terrestrial Reference System (ITRS) describes procedures for creating reference frames in their usable form, such as the International Terrestrial Reference Frame (ITRF). This global reference frame is used to reference the motion of the Earth's crust and is considered the basis for computations that are transformed to a geodetic reference system, such as the North American Datum for the United States.

The definition of the geoid is adopted from the National Geodetic Survey (NGS) and is stated as "the equipotential surface of the Earth's gravity field which best fits, in a least squares sense, global mean sea level" (http://www.ngs.noaa. gov/GEOID/geoid_def.html). It is important to distinguish between ellipsoid and geoid heights to understand how elevations are derived. GNSS observations measure the distance above or below the ellipsoid reference surface, defined as ellipsoid heights $(h)$ (fig. 1). These ellipsoid heights are converted to orthometric heights $(H)$ or elevations by the separation distance between the ellipsoid and the geoid $(N)$ (fig. 1).
The separation distance, or geoid height, is positive away from the Earth center and negative towards it (Henning, 2010). In the conterminous United States, the geoid is always a negative value, such that the geoid surface is below the ellipsoid surface. Referring to figure 1, an example calculation may be indicated as follows: A GNSS observation taken at $37^{\circ} 08^{\prime} 23.64535$ north and $-93^{\circ} 26^{\prime} 28.22070$ west computes a height of 361.756 meters. The geoid height located at this latitude and longitude is -29.24 meters. The elevation is:

$$
H=h-N
$$

where

$$
\begin{aligned}
H & =\text { orthometric height } \\
h & =\text { ellipsoid height, and } \\
N & =\text { geoid height, therefore } \\
H & =361.756-(-29.24)=390.996 \text { meters }
\end{aligned}
$$

In summary, GNSS observations are measurements of ellipsoid height which are converted to orthometric heights (elevations) using a hybrid geoid model based on a network of known vertical benchmarks (Henning, 2010). Currently, the 2009 geoid model (GEOID 09) is the most current hybrid geoid model used in the conterminous United States; however, new geoid models will be produced in the future to update physical characteristics of the Earth. New geoid models are produced out of necessity as plate tectonic shifting, subsidence, and anthropogenic disturbances alter the coordinates of physical survey monumentation used to develop these models.

A point of concern about the usefulness of a hybrid geoid model is the accuracy of the model for the area in which a GNSS campaign is led. A comparison between the number of locations in each state for which a GNSS ellipsoid height and a leveled NAVD 88 orthometric height is known (thus defining the separation distance) and the 2009 geoid model (GEOID 09) for that state is represented in table 2. This table essentially represents an indication of geoid accuracy for each state. The standard deviation in table 2 is expressed at the 68-percent confidence level; therefore, each value should be doubled to achieve a more confident measure (95-percent confidence interval). For example, in the state of Missouri, one could generally expect a standard deviation of 2 centimeters $(\mathrm{cm})$ (95-percent confidence level) from the modeled geoid as compared to 138 benchmarks (or known separation distances) consisting of GPS-derived ellipsoidal height and leveled orthometric height data (Roman and others, 2009). Continuing with the example; the quality of the geoid model appears to be slightly more favorable in Missouri as compared to Georgia, where the standard deviation from nearly the same number of benchmarks is $0.8 \mathrm{~cm}$ greater in Georgia $(2.8 \mathrm{~cm}$ at 95-percent confidence level) than Missouri.

To fully understand how orthometric heights are referenced, the geodetic reference system containing horizontal and vertical datum must be defined. The official national geometrical datum for the United States is the North American Datum of 1983 (NAD 83), which is usually expressed in 


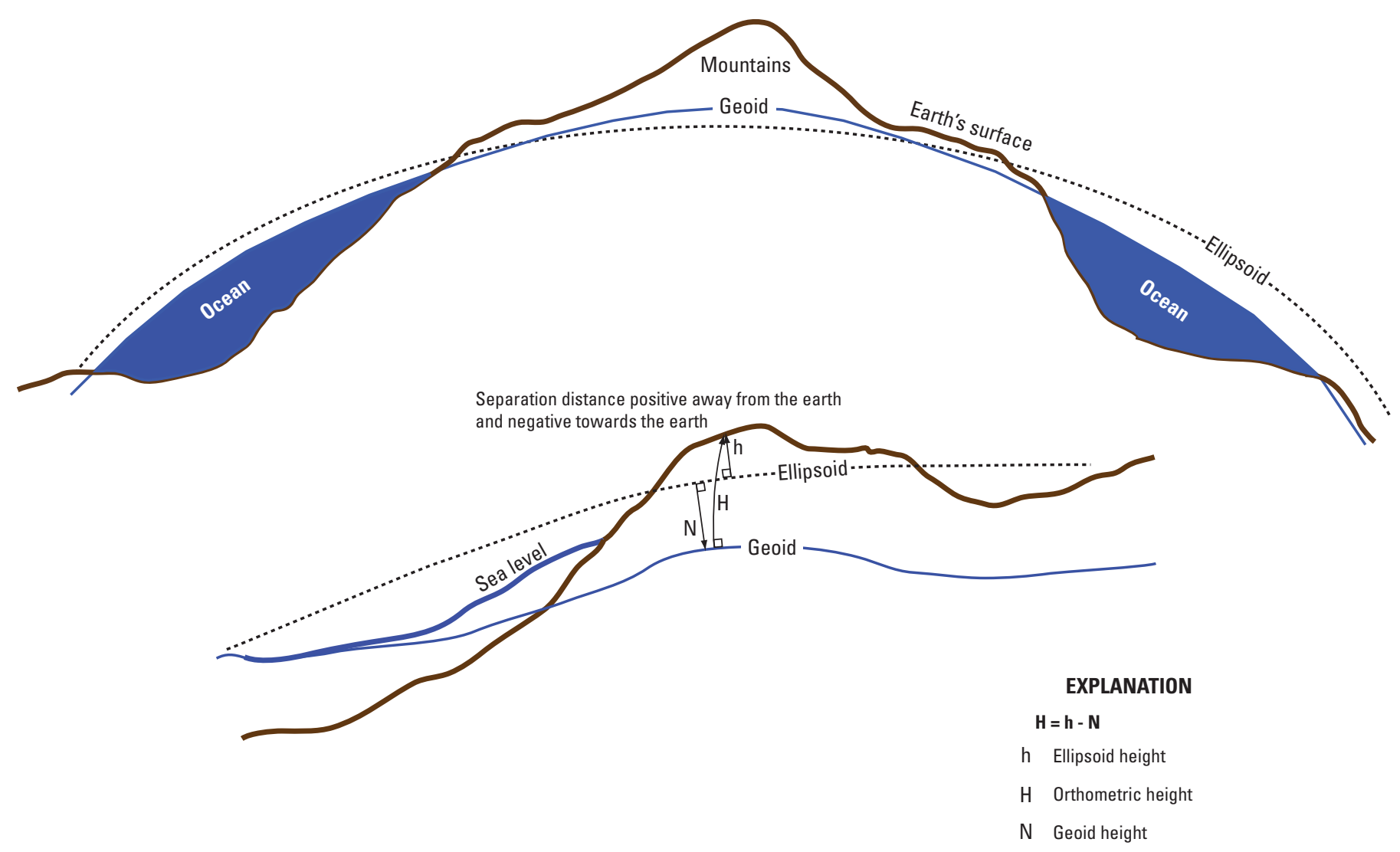

Figure 1. Relation between the ellipsoid, geoid, and orthometric heights.

three dimensions as latitude, longitude, and ellipsoid height (Henning, 2010). This Earth-centered datum has become more refined and has been adjusted as a result of satellite and gravity measurements that contributed to the level of precision. The NAD 83 datum adjustments are labeled with a certain time stamp, or "epoch," a reference frame denoted by a particular year. The most current realization that is generally used throughout the United States is the NAD 83 (CORS96), epoch 2002.0, which constitutes the framework for the definition of the National Spatial Reference System (NSRS) (Soler and Marshall, 2003). This realization is most commonly adopted in real-time or post-processed positioning. An example of a NAD 83 realization, as defined by Henning (2010) as its physical, useable manifestation of this datum, is NAD 83 (NSRS2007), an approximation for the distribution at nearly 70,000 geodetic control monuments. A realization of the passive marks that occurred January 1, 2012, used the multi-year CORS adjustment that combines global stations and CORS to assess positional differences resulting from velocities owing to crustal motion (Stone, 2011). This realization is known as NAD 83 (2011), epoch 2010.0 for the United States including Alaska, Puerto Rico, and the Virgin Islands. The realization for the Pacific tectonic plate (including Hawaii) is NAD 83 (PA11), epoch 2010.0.

Once the earth centered, earth fixed (ECEF) " $x, y$ and z" coordinates derived from GPS are transformed to the NAD 83 values with ellipsoid heights, an application of the current hybrid geoid model yields an orthometric height referenced to the official national vertical datum of the United States, better known as the North American Vertical Datum of 1988 (NAVD 88). The NAVD 88 datum is based on the height of the primary tidal bench mark referenced to the new International Great Lakes Datum of 1985, representing local mean sea level at Father Point/Rimouski, Quebec, Canada (National Geodetic Survey, 2010a). This height was held fixed during a minimumconstraint adjustment of leveling observations that were applied throughout the United States, Mexico, and Canada. The NAVD 88 vertical datum is applicable to the conterminous United States and Alaska, but there are other vertical datum used, such as the Puerto Rico Vertical Datum of 2002 (PRVD 02) in Puerto Rico and a refined gravimetric model of the geoid (USGG 2009) in Hawaii (offset by $50-60 \mathrm{~cm}$ from some local tidal benchmark values), that are applied as the vertical datum surface.

\section{Purpose and Scope}

This manual outlines approaches and guidelines to ensure quality survey-grade GNSS surveying when establishing vertical datum for various applications within the USGS. The focus of this manual is on the application and execution 
Table 2. Residual differences between the Geoid 2009 (GEOID 09) hybrid model and known separation distance as determined from Global Positioning System (GPS)-derived ellipsoid heights and leveled North American Vertical Datum 1988 (NAVD 88) orthometric heights at benchmarks within the conterminous United States.

[Modified from Roman, 2009. m, meter]

\begin{tabular}{|c|c|c|c|c|c|c|c|}
\hline State & $\begin{array}{c}\text { Number of } \\
\text { GPS } \\
\text { benchmarks }\end{array}$ & $\begin{array}{c}\text { Average } \\
\text { bias } \\
\text { (m) }\end{array}$ & $\begin{array}{c}\text { Standard } \\
\text { deviation } \\
(\mathrm{m})\end{array}$ & State & $\begin{array}{c}\text { Number of } \\
\text { GPS } \\
\text { benchmarks }\end{array}$ & $\begin{array}{c}\text { Average } \\
\text { bias } \\
\text { (m) }\end{array}$ & $\begin{array}{c}\text { Standard } \\
\text { deviation } \\
\text { (m) }\end{array}$ \\
\hline Alabama & 283 & 0.000 & 0.011 & Nebraska & 145 & 0.000 & 0.007 \\
\hline Arizona & 227 & 0.000 & 0.016 & Nevada & 70 & 0.001 & 0.012 \\
\hline Arkansas & 133 & 0.001 & 0.018 & New Hampshire & 14 & -0.003 & 0.009 \\
\hline Connecticut & 20 & 0.000 & 0.015 & New York & 185 & 0.000 & 0.011 \\
\hline Delaware & 35 & 0.001 & 0.012 & North Carolina & 1,676 & 0.000 & 0.015 \\
\hline District of Columbia & 16 & 0.004 & 0.020 & North Dakota & 47 & 0.001 & 0.007 \\
\hline Florida & 2,181 & 0.000 & 0.014 & Ohio & 297 & 0.000 & 0.022 \\
\hline Indiana & 119 & 0.000 & 0.013 & Rhode Island & 29 & 0.000 & 0.018 \\
\hline Iowa & 100 & -0.001 & 0.009 & South Carolina & 1,315 & 0.000 & 0.012 \\
\hline Kansas & 105 & 0.000 & 0.009 & South Dakota & 242 & 0.000 & 0.008 \\
\hline Kentucky & 123 & -0.001 & 0.013 & Tennessee & 302 & 0.000 & 0.018 \\
\hline Louisiana & 217 & -0.001 & 0.012 & Texas & 218 & 0.000 & 0.012 \\
\hline Maine & 65 & 0.000 & 0.011 & Utah & 55 & 0.000 & 0.016 \\
\hline Maryland & 511 & 0.000 & 0.016 & Vermont & 317 & 0.000 & 0.013 \\
\hline Massachusetts & 35 & 0.000 & 0.012 & Virginia & 434 & 0.000 & 0.021 \\
\hline Michigan & 410 & 0.000 & 0.015 & Washington & 259 & 0.000 & 0.017 \\
\hline
\end{tabular}

of GNSS as it pertains to the USGS. Fundamentals, including operational theory, firmware algorithms, and detailed geodesy are left to other well-documented sources, more specifically those disseminated by the National Geodetic Survey (NGS) at www.ngs.noaa.gov. This manual also focuses on quality-assurance measures while achieving the maximum efficiency of a GNSS campaign to establish vertical datum. Different quality-control standards may apply to the establishment of vertical datum for special investigative studies in comparison to USGS standardized data collection efforts on a nationwide scale. This manual recognizes GNSS strategies that are largely dependent upon the quality of the datum to be established (as dictated by the overall science), supporting benchmarks, and the spatiality of the campaign. Typical scenarios for which GNSS surveys are undertaken in the USGS are investigated and an approach that ensures quality and efficiency is formulated. Note that the term Global

Positioning Systems or GPS is used in this manual to define the U.S. space-based system (U.S. GPS) of global positioning exclusively, whereas the term "Global Navigation Satellite Systems" or "GNSS" represents satellite constellations available throughout space, including the U.S. GPS. To avoid confusion, the term "Global Navigation Satellite System" or "GNSS" will be used when describing the technology and system of terrestrial positioning with satellite signals, unless specifically referring to the U.S. GPS system.

\section{Equipment}

GNSS equipment is composed of different components, such as receivers, antennas (receiver and radio), tripods, tribrachs, radios, data collector, software, batteries, and 
connective cables that permit the system to function. Variations of GNSS equipment components are contingent upon the type of survey to be led and whether one will use postprocessed or real-time methods.

Post-processed methods of GNSS surveying are generally referred to as static surveys. These surveys are not mobile and collect data at the receiver to be processed at a later date. Data collection occurs within the receiver that is mounted on a tripod over a point of interest. Typically, occupation times range anywhere from 15 minutes for rapid-static sessions to as much as 24 hours for longer static sessions. Applications and approaches of post-processed methods of global positioning will be described in greater detail in the "Static GNSS" section of this report.

For post-processed methods, equipment needs are simplified. Essentially a receiver with a receiver antenna and power supply is needed on top of a tripod. In many cases, a tribrach is used to attach and level the receiver to the tripod. The tribrach has an adjustable top plate that can be leveled by way of three thumb screws relative to a fixed bottom plate that attaches to the top of the tripod (fig. 2). A data collector is typically used to initiate and conclude the observation session, although internal configuration files often exist that can be uploaded to a receiver to start and stop logging data when powering up and powering down the receiver.

There are generally two methods involving real-time (RT) GNSS surveying. The traditional method, known as single-base Real-Time Kinematic (RTK) surveying, is a global positioning approach that involves a single stationary receiver (known as a base station) that provides a real-time differential correction from signals received at the base, applied to those signals received by a mobile unit (known as a rover) by way of radio. Single-base RTK methods involve equipment such as a fixed-base receiver in addition to radio and accessories, including an external radio whip antenna, power supply, and support tripod. Another RT method involves a network of fixed receivers known as a Real-Time Network (RTN), accessible by wireless communication. These RTNs use a centralized server to facilitate quality-assurance checks, network modeling, estimation of systematic errors, and calculation of corrected data that is submitted back to the end user (rover). During a RTN survey, the radio and accessories are omitted because the network of fixed receivers is linked through the application of wireless broadband. RTN surveys often do not require an additional fixed-base receiver because the network itself provides the needed real-time corrections and coordinate basis. Applications and approaches of both real-time methods of global positioning will be described in greater detail later in this manual.

\section{Overview and Function}

Function and detail of GNSS equipment electronics and GNSS radio communication is well documented in other sources and will not be described in this text; however, it is important for the user to recognize the general purpose and limitations of GNSS equipment to plan appropriately for a specific campaign. The primary components of a GNSS system are the receiver, receiver antenna, radio, radio antenna, data collector, tripod, and software.

\section{Receivers}

The receiver is the most integral part of global positioning infrastructure. Survey-grade receivers are typically dual frequency, defined as utilizing satellite signals frequencies in the L-band at 1575.42 Megahertz $(\mathrm{MHz})$ for $\mathrm{L}_{1}$ and $1227.60 \mathrm{MHz}$ for $\mathrm{L}_{2}$. Most receivers have power-input levels that vary from 10.5 to 28 volts (Henning, 2010). Dual-frequency receivers are known for their resolution of ionospheric delay. Ionospheric delay is an important concept

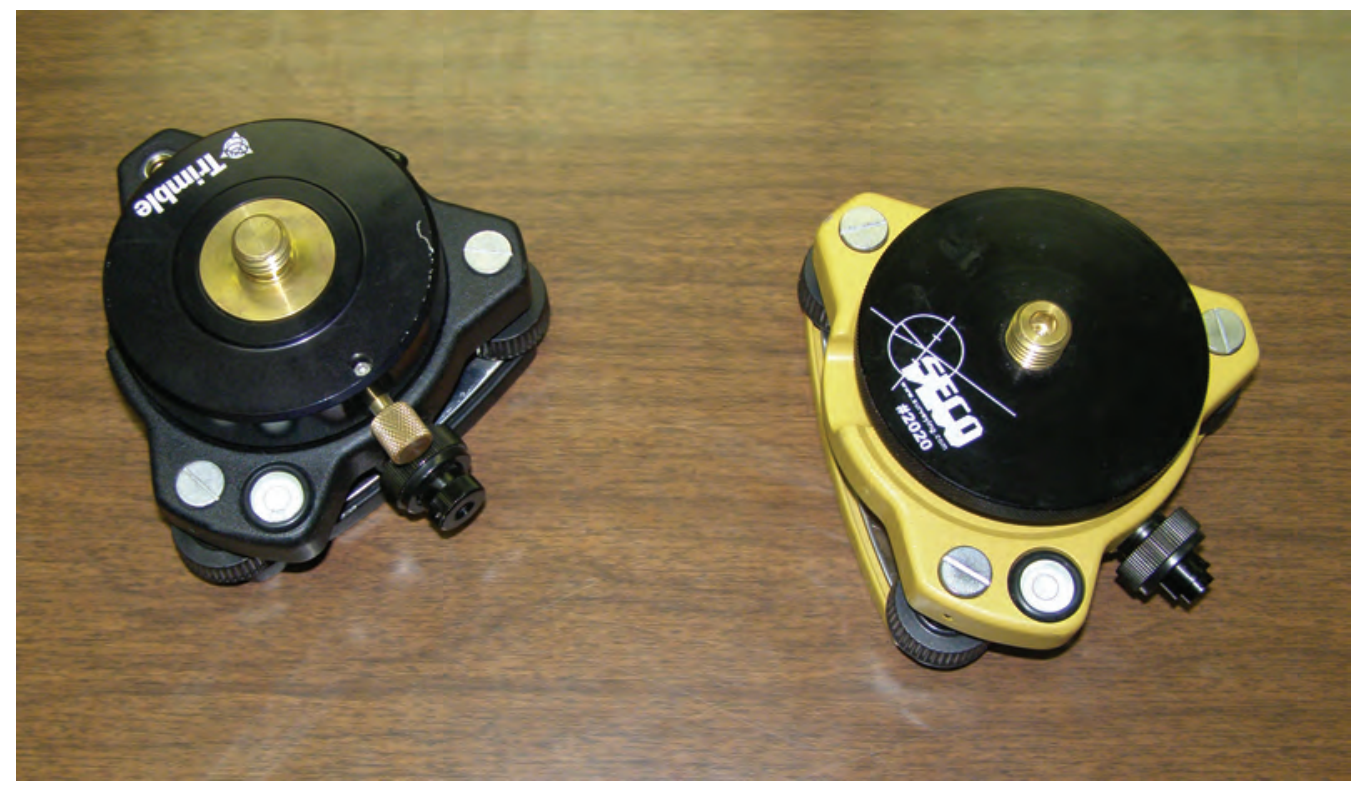

Figure 2. Tribrachs used with tripods to attach Global Navigation Satellite System (GNSS) receivers. 
to GNSS surveys, defined as the change in speed in which signals are transmitted through the electron- and ion-charged atmosphere between 40 and 400 miles above the Earth's surface. Changes in signal speed (most commonly a delay) through the ionosphere are usually the result of energetic events from the sun, and a dual-frequency receiver is beneficial because it uses differences between the frequencies to correct the resulting error. Dual-frequency receivers permit longer baselines (distances) in addition to shorter times of satellite acquisition and trilateration (initialization). At this time of this writing, receivers are being manufactured with the allowance for a third frequency, known as $\mathrm{L}_{5}$, and other enhancements ( $\mathrm{L}_{1} \mathrm{C}$ and $\mathrm{L}_{2} \mathrm{C}$ with optimized signal design) to existing signals $\mathrm{L}_{1}$ and $\mathrm{L}_{2}$ as part of an ongoing effort to modernize GPS with new advanced capabilities to meet military, civil, and commercial needs (U.S. Department of Commerce, 2010). The new civilian signal $L_{5}$ provides a higher power level than other signals and has a greater bandwidth, which makes it easier to acquire and track weaker satellite signals. Another classification of receiver is one that has the capacity of augmenting GPS signals with signals from the GLONASS and Galileo constellation of satellites (table 1). Although many existing dual-frequency receivers are only equipped for GPS signals, many (including multi-frequency receivers) are compatible to receive signals from GLONASS and Galileo (Galileo will have the $\mathrm{L}_{5}$ availability; NavtechGPS, 2012). As future constellations are made available for civilian use, receivers will be manufactured to ensure the greatest benefit of signal tracking.

It is important to upgrade firmware and maintain current versioning with any GNSS receiver. Firmware upgrades are offered to increase performance and usability. GNSS manufacturers usually have a web link that offers the upgrades, and it is the user's responsibility to ensure installation of these upgrades as they become available. These upgrades offer many benefits, including improved radio communication range, optimized storage and data transfer, wireless enhancements, and assorted handling of satellite messages.

\section{Antenna (Receiver and Radio) and Radio}

Receivers may have an external or an internal antenna that holds the electronic phase center to which a baseline vector is computed to form a baseline solution. This antenna receives satellite signals that include the time the message was sent, orbital information of the GNSS satellite network, and general system health of all GNSS satellites. The antenna of a GNSS receiver is identified as "micro-centered," which means that there is no specific orientation that needs to be preserved once established in the field, unless explicitly specified by the manufacturer. There are several different types of antennas that have varying degrees of "on-board" signal qualityassurance capabilities; examples of different types of GNSS receiver antennas are depicted in figure 3.

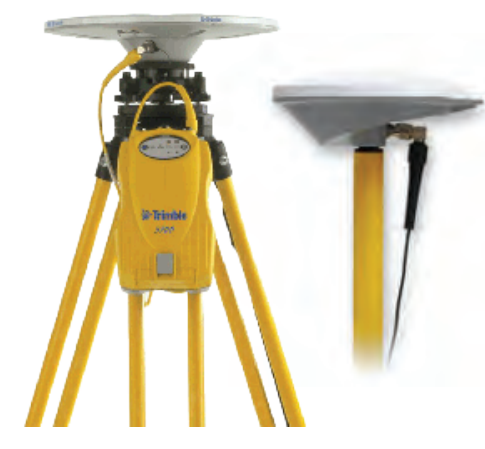

Receiver and micro-centered antenna with and without prominent ground plane.

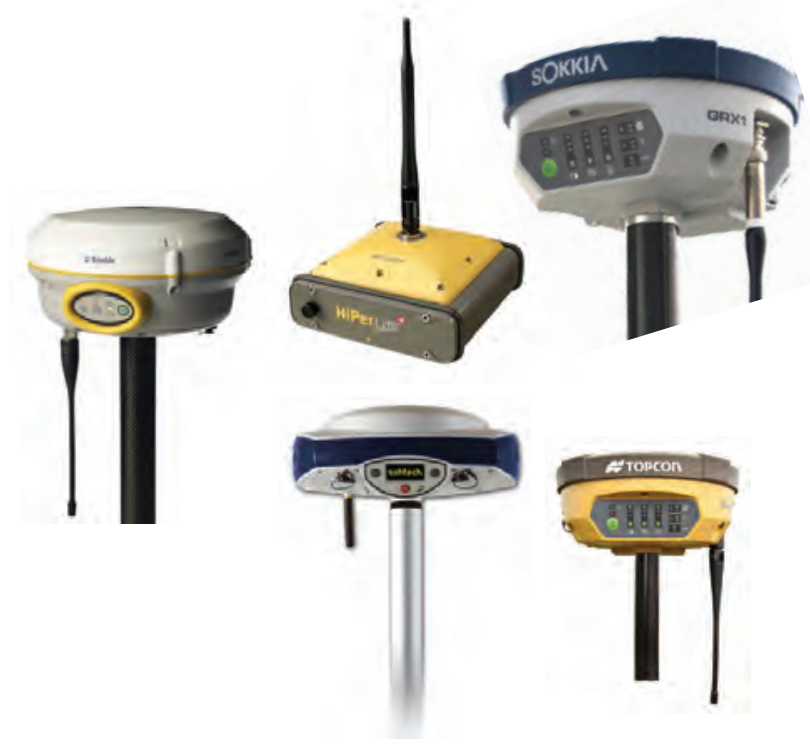

Integrated recievers and antennas with micro-centered built-in ground plane.

Figure 3. Various types of Global Navigation Satellite System (GNSS) receiver antennas.

A radio link is typically used when employing a singlebase RTK approach, which requires a broadcast radio and antenna at the base receiver location, and a receiving radio and antenna at the rover location; however, many single-base real-time approaches employ a data modem for the wireless communication method of choice. This radio or modem system communicates and maintains a differential correction "on-the-fly" during these surveys. A longer whip antenna or an optional extended range antenna pole will enhance communications by ensuring a higher signal to noise ratio (Henning, 2010). Antenna enhancements can be made at the base receiver, rover receiver, or both (fig. 4). During single-base RTK campaigns with long baselines, radio communications have been substantially improved by incorporating an extended range pole antenna at the rover unit. For linear surveys without obstructions, a directional antenna for the broadcast radio may be considered (Henning, 2010). 


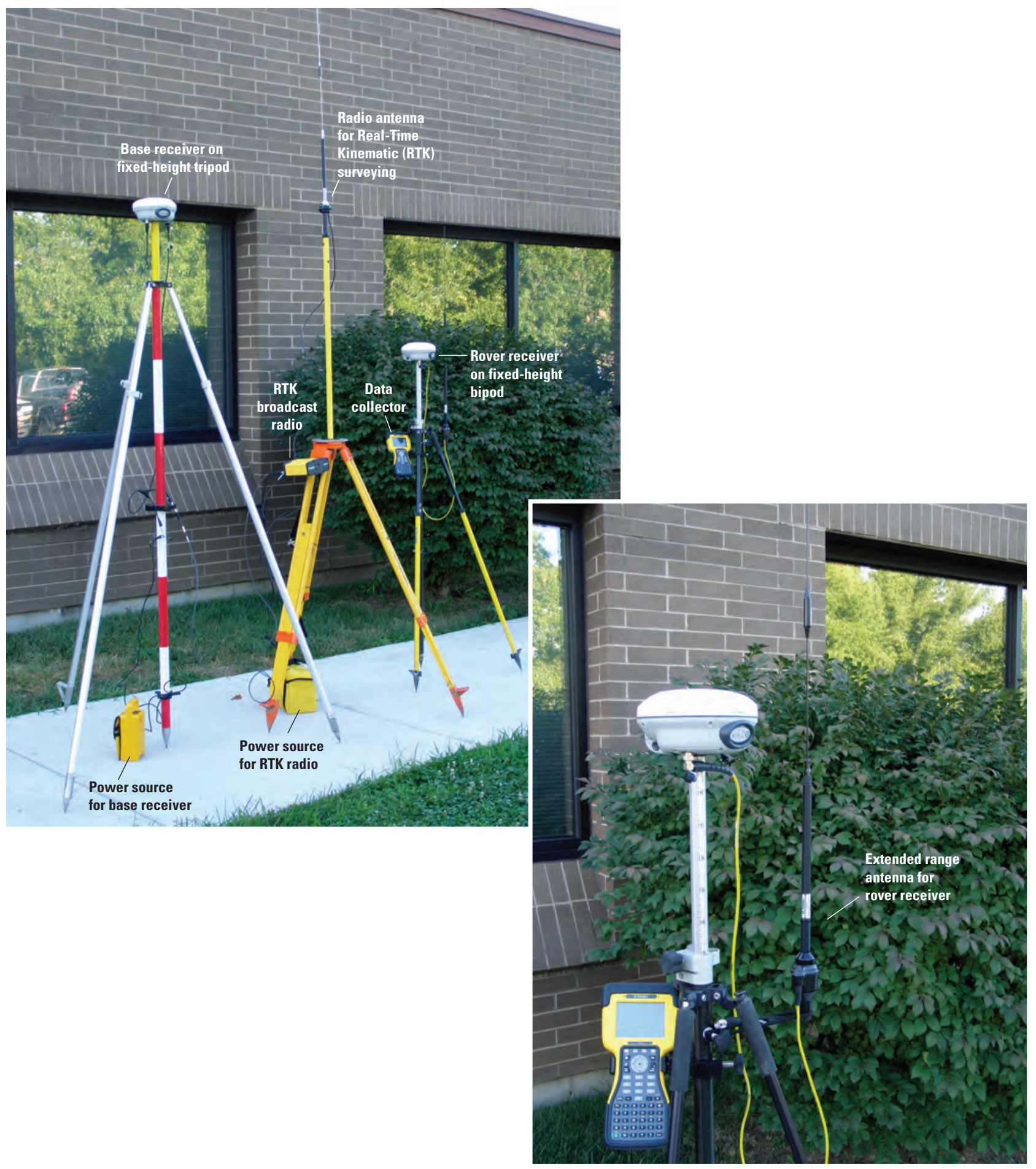

Figure 4. Traditional real-time equipment profile. Note extended range antenna enhancement for rover receiver. 
The required wireless communication can be a source of frustration when communications fail. For radio communication, there are five factors, derived from the Pacific Crest Corporation (2009) that should be analyzed to ensure radio communication and range:

1. Environment-

- Assessment of radio range as comparing flat or hilly terrain, with or without buildings and trees; and

- Proximity of radio towers or airports as other radar and radio emissions can greatly reduce range.

2. Antenna-

- "Mobile whip" antennas for RTK surveying (fig. 4) out-perform "rubber duck" antennas (fig. 5);

- Upper and lower frequency range limits of the antenna should be equivalent to that of the radio;

- Upper and lower elements of the whip antenna should have the same gain (that is $0 \mathrm{db}$ or $5 \mathrm{db}$ gain); and

- Elevate antenna as high as possible (usually with an antenna mast). Studies have indicated that doubling the antenna height will increase the range by 40 percent (Henning, 2010).

\section{Antenna cables-}

- Shorter cable lengths promote better range because losses are minimized. For extended antenna heights, a low-loss cable should be used;

- Cable types of LMR200 are more efficient than RG58; and

- Cable damage can be determined by swapping antenna cables.

4. Battery cable and connectors-

- Battery age (commonly replace after 2 years);

- Extreme hot or cold temperature will reduce the power output of the battery;

- Condition of the battery cable; a frayed or cracked cable may fail to deliver full power;

- Condition of battery connectors; assure periodic cleaning with a brush and contact cleaner every few months; and

- Clip usage; battery may fail to deliver full power when using alligator clips.

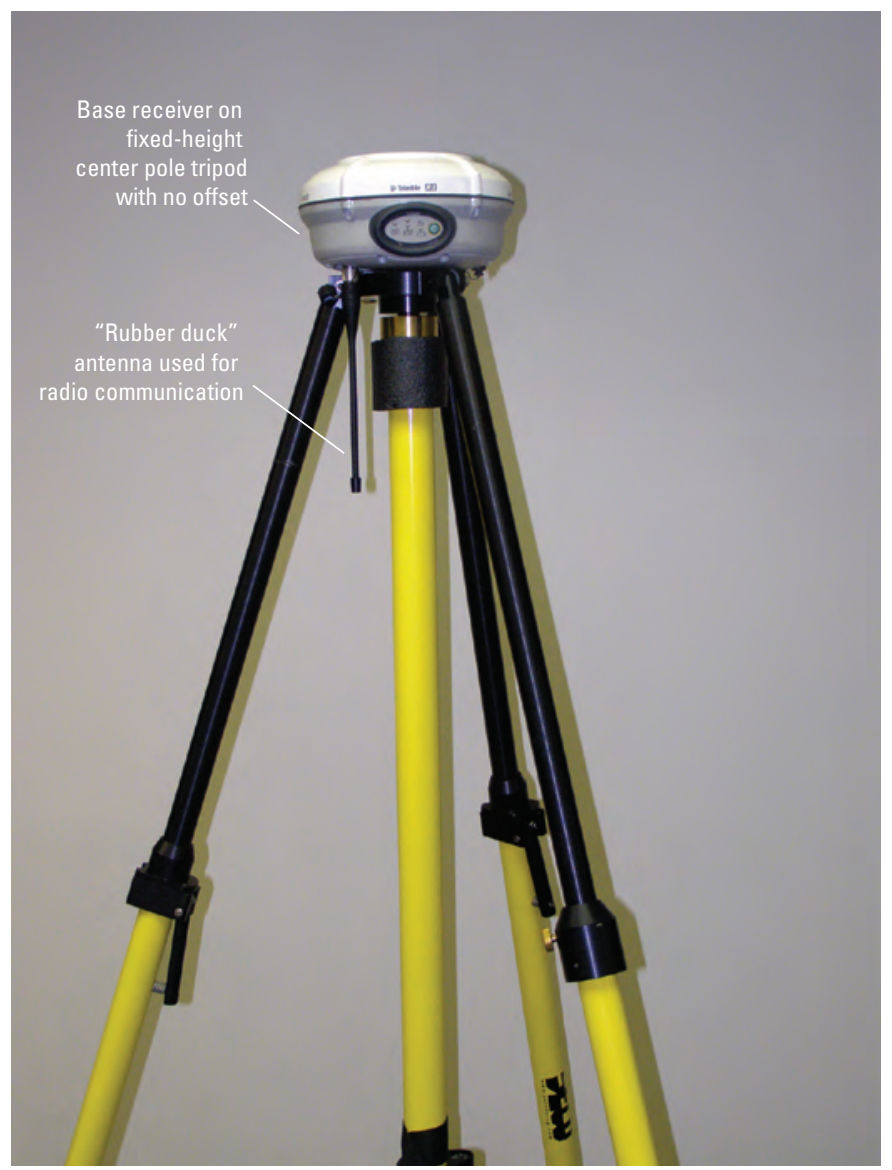

Figure 5. "Rubber duck" antenna used for radio communication.

5. Broadcast radio-

- Use the latest firmware release in the radio; and

- Assure that the digisquelch or sensitivity is appropriately set to "low" or "high," depending upon the application (receiving signals over short or long distances) and potential for any external radio interference.

The GNSS broadcast radio used during single-base RTK surveys should maintain a 12-volt supply at all times. Base radios usually vary from 25 to 35 watts and have a typical range of 3 to 5 miles (Henning, 2010). For lengthy surveys involving single-base RTK positioning, a secondary radio may be considered to be used as a repeater along a particular baseline. A repeater radio is simply used to pick up the base station radio signal and re-broadcast the signal to the rover receiver over a longer baseline distance with minimal latency.

For RTN surveys, the GNSS base receiver and broadcast radio are always omitted, and a wireless broadband or cellular modem is substituted to communicate with the network over the internet using cellular networks. Nevertheless, it is good practice to include the base receiver and a broadcast radio as a "back-up" for occurrences where broadband or cellular communication is lacking; however, there are devices such as a 
RTK bridge (fig. 6) that can be used to interface to the internet through a cellular modem, established connectivity with a RTN server, and authenticate and broadcast correction messages to the field rover by way of radio. Devices such as these are useful for areas void of cellular coverage while permitting multiple rover units without additional cellular modems.

For GNSS radios, it is important to identify the frequency band in which the USGS is authorized to operate as a federal agency. The range for survey-grade GNSS is between $410 \mathrm{MHz}$ and $420 \mathrm{MHz}$ (R. Pardee, U.S. Geological Survey, written commun., Feb. 24, 2011). It is therefore important to convey to vendors and procurement officers the permissible range before purchasing equipment and ensuring the acquisition of special certifications that may be applicable.
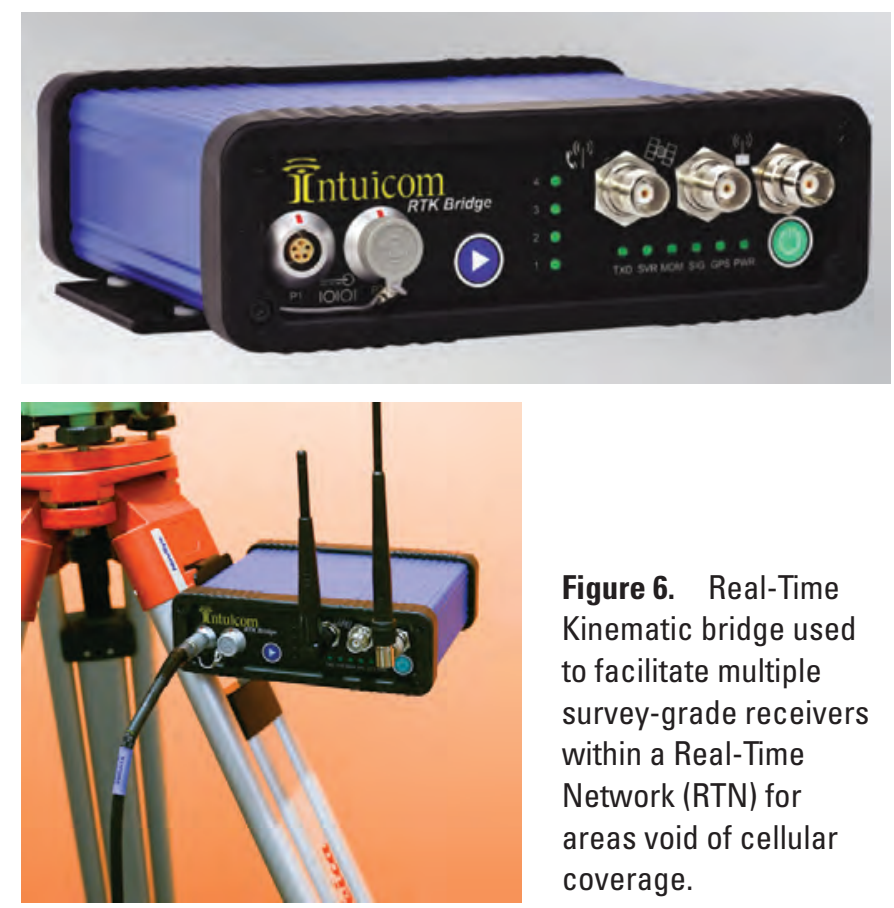

Figure 6. Real-Time Kinematic bridge used to facilitate multiple survey-grade receivers within a Real-Time Network (RTN) for areas void of cellular coverage.

\section{Data Collector}

The data collector serves as an interface to communicate with receivers to initiate a GNSS observation (fig. 7). In addition, data collectors facilitate satellite tracking, data storage, processing, dissemination, transformations between datum and projections, and provide other quality-assurance diagnostics. Data collectors communicate either by data cable or wireless. Most data collectors possess features such as enhanced graphics, large memory capacities, and tough weather-resistant construction. Data collectors should be used to provide quality assurance during a campaign by reviewing and contrasting redundant observations, and often possess functionality used for various coordinate geometry computations. Although data collectors minimize the need for hand-written notes in the field, it is considered good general practice to document primary aspects of a GNSS campaign in hand-written form that is well organized in a field notebook. These aspects will be detailed in the "Metadata" section.
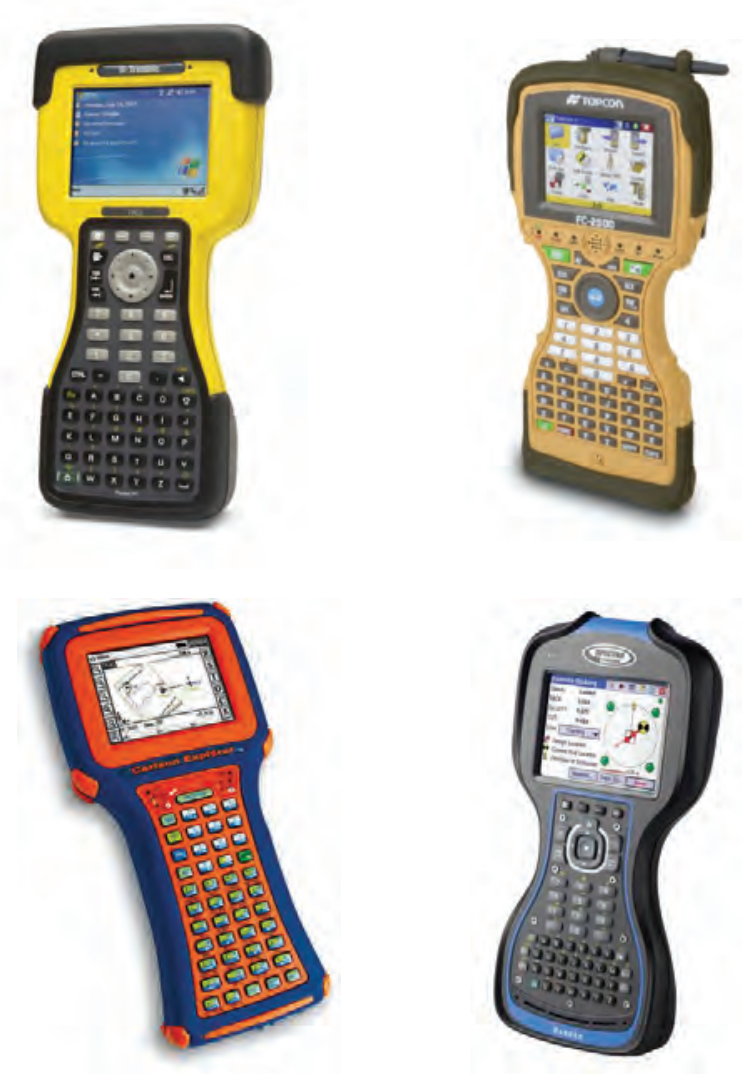

Figure 7. Data collectors used for Global Navigation Satellite System (GNSS) positioning.

\section{Tripods}

A fixed-height tripod is recommended for all GNSS receivers in the static mode and the base receiver for RT. The fixed-height tripod essentially has a centered fixed-height range pole (usually 2 meters) that is supported by three adjustable external legs (fig. 8). Many tripod assemblies have an adjustable center pole with fixed-height dowel stops. During a single-base RTK survey, a fixed-height, single-carbon, fiber range pole with associated bipod is often used with the rover receiver (as opposed to the fixed-height tripod used for the base receiver). Similar to fixed-height tripods, some bipod assemblies have an adjustable center pole with fixed-height stops. Several manufacturers have been developing fixedheight tripods and bipods for the purpose of providing stability by prohibiting movement of the center pole. Additionally, these fixed-height platforms provide assurance for antenna centering, prevent height measurement error, and reduce setup time. Traditional tripods are not as vertically stable as tripods with fixed-height center poles, and are not recommended for GNSS receivers because they are more likely to be subject to movement for long observations; however, a traditional tripod that employs dual clamps may be considered for base stations during RT observations or static observations. Dual clamps are usually a combination of wing screws and quick 


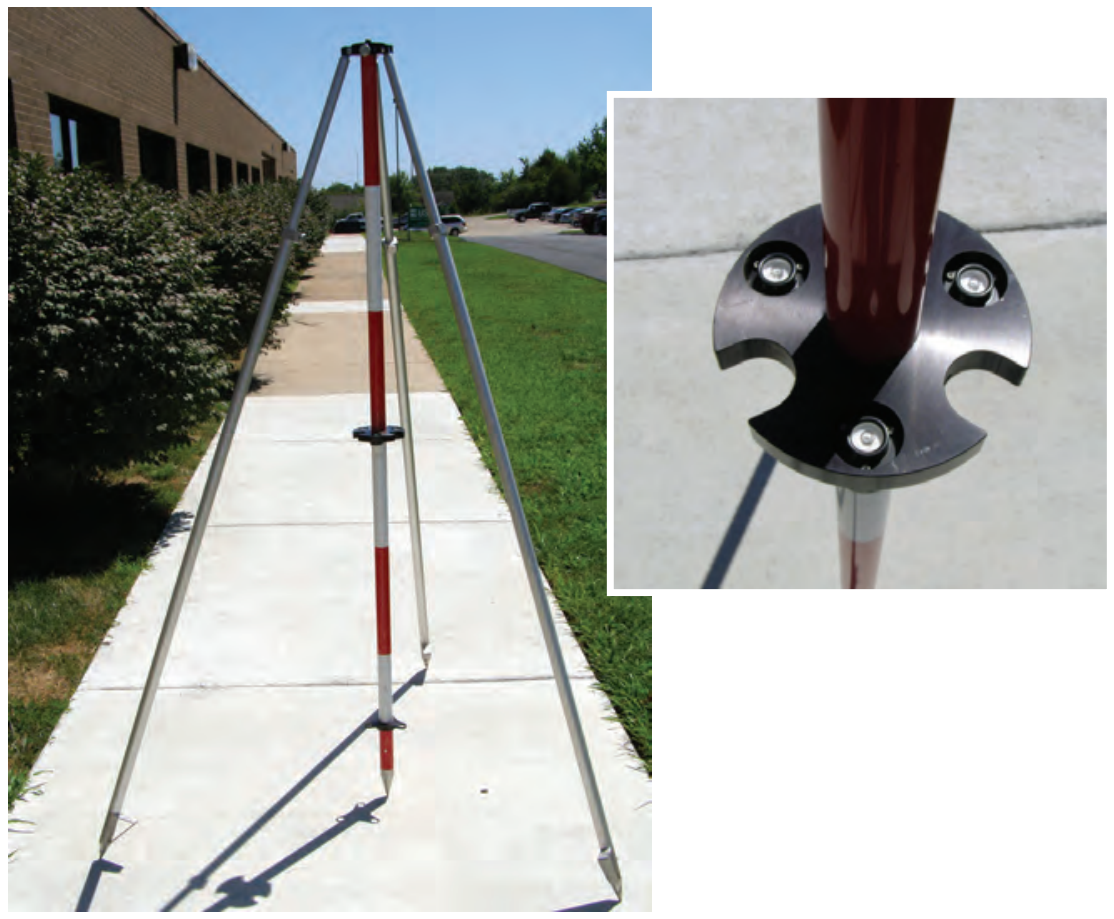

Fixed-height center pole tripod.

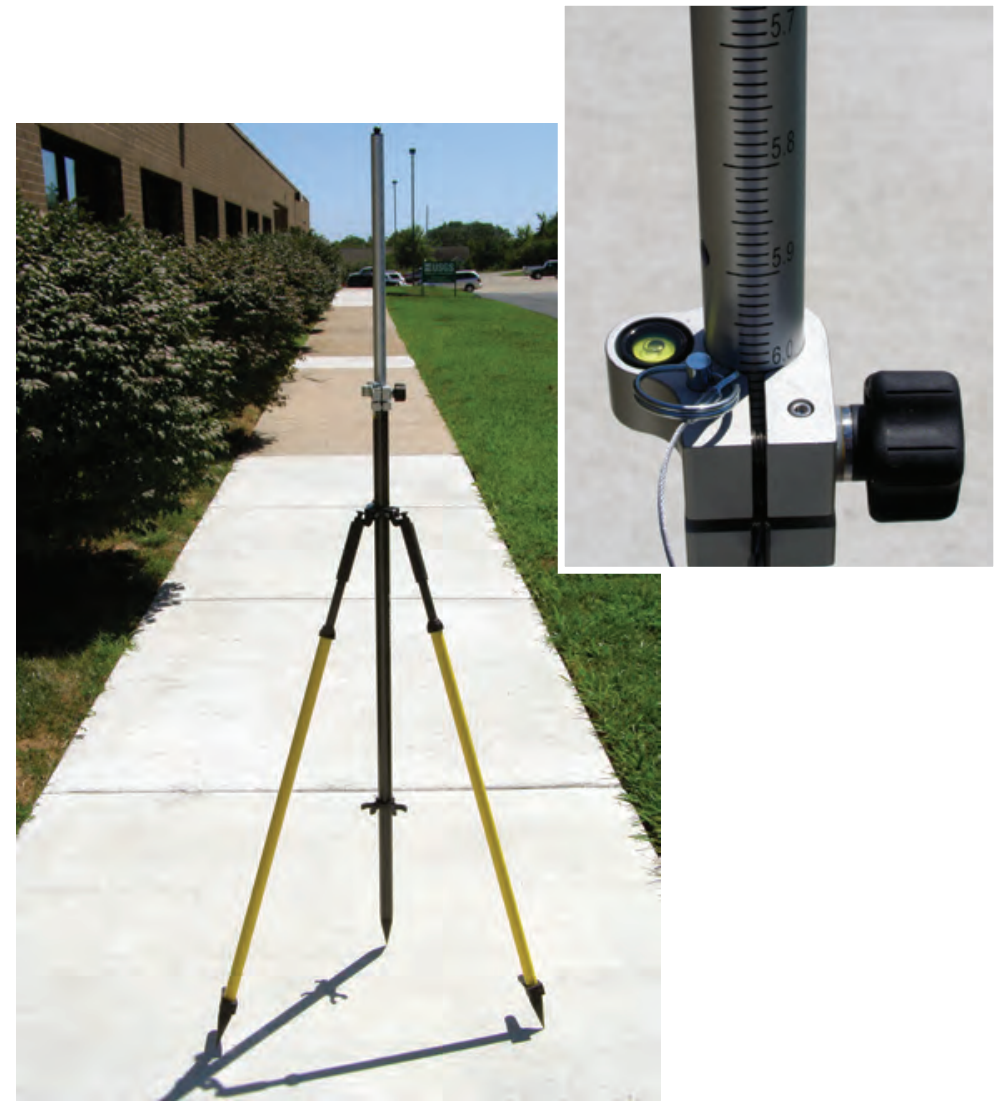

Adjustable center pole with fixed-height stops, supported by a bipod.

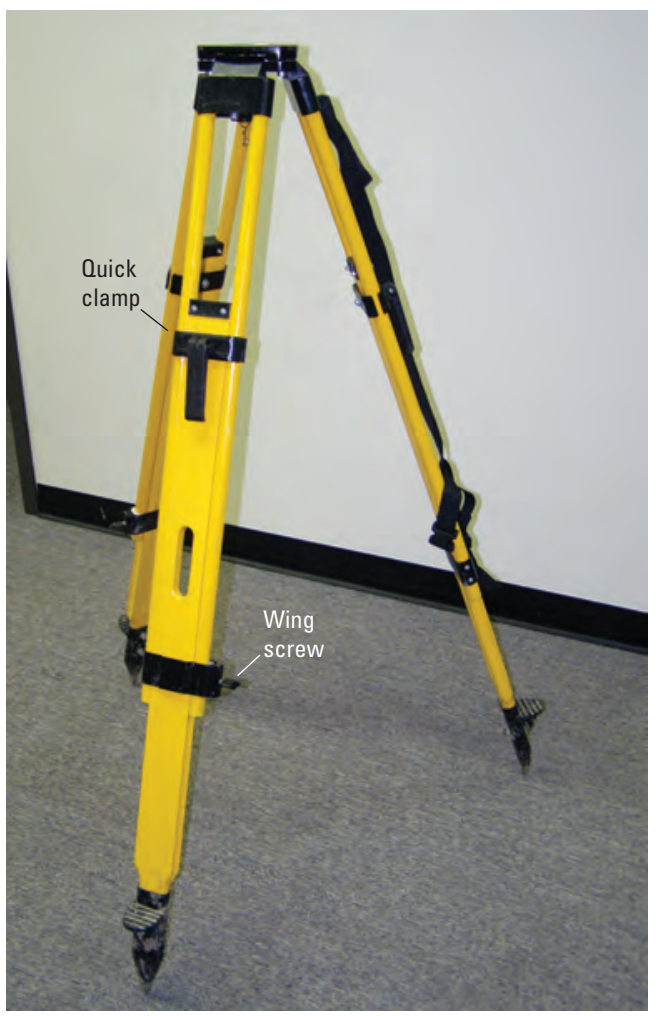

Traditional tripod with dual clamps. May be used for Global Navigation Satellite System (GNSS) receivers.

Figure 8. Fixed-height and adjustable fixed-height center pole tripod and bipod used for Global Navigation Satellite System (GNSS) positioning. 
clamps (fig. 8). Other traditional tripods may be used for a broadcast radio and antenna setup, as this use does not require a high degree of stability. When using a traditional tripod, it should be in good condition such that all legs, hinges, clamps, and feet are secure and functioning.

Circular bubble level vials, or "bulls eye" levels, are located on the fixed base tripod and rover bipod. It is good practice to ensure an adequate plumb of the circular level vial (and adjust if necessary by evaluating the bubble location before and after a 180 degree rotation of the fixed center pole (fig. 9). Figure 9 illustrates a recommended procedure documented by Henning (2010). This procedure is only applicable for fixed-height tripods with rotating center poles, or a fixed-height pole removed from a set of bipod legs that could be used in a door jam.

\section{Benchmarks}

For any GNSS survey campaign, a proper benchmark is essential to preserve measurement location and elevation. Historically, leveling field operations for second- and thirdorder geodetic leveling, provided in the topographic instructions of the USGS, distinguished survey benchmarks as either monumented or non-monumented benchmarks (U.S. Geological Survey, 1966). Examples of monumented and non-monumented benchmarks are described in figure 10. Monumented benchmarks have a tablet consisting of identifying information surrounding a stamped center point. These marks are represented as a standard metal tablet, disk, cap, or steel rod used to describe the elevation. These tablets are commonly set in concrete, stone posts, firm rock outcroppings, masonry structures, and buildings (U.S. Geological Survey, 1966). Another unique monumented benchmark that may be used in areas void of rock substrate is known as a FENO marker. FENO markers utilize 1-meter long stakes with extendible anchors at the bottom to keep them secure (fig. 10). FENO markers are monumented benchmarks because a tablet is secured to the top of the stake. If monumented benchmarks are selected to be used, they must be marked with identifying information, generally the agency setting the benchmark plus other information that the agency can use to uniquely identify the benchmark [NGS uses a Permanent Identifier (PID) and designate, whereas the USGS Nebraska Water Science Center uses a four-digit numbering system]. In addition, monumented benchmarks are often stamped with elevation and the established year.

The selection of benchmarks used in a GNSS campaign is critical to ensuring quality orthometric heights. As defined later in the Real-Time and Static GNSS sections, there are many different quality indicators and assurances that can be taken to produce heights with minimal uncertainty; one of the most fundamental assurances is the acceptance of a benchmark that is "trusted" with its subsequent use as "truth" in evaluating the quality of the campaign. The decision to "trust" a benchmark must be made after a thorough evaluation of the history and stability of the mark as well as any plate tectonic shifting, subsidence, or heave that may depreciate confidence in the mark in the area of recovery. Using the benchmark as "truth" simply identifies the mark or set of marks as absolutes that dictate the uncertainty of the effort.

Non-monumented benchmarks (also referred to as "temporary," "intermediate" or "supplementary") may consist of any of the following: chiseled squares; crosses or circles on concrete or masonry structures; bolt heads in steel, concrete, or masonry structures; and metal pins or magnetic (mag) nails in concrete or asphalt (U.S. Geological Survey, 1966) (fig. 10). Non-monumented benchmarks are simply a mark with no identifying information.

For GNSS campaigns within the USGS, the vertical component of positioning is usually the primary concern. Stability and an open view of the sky are key considerations for benchmarks. The identification of the frost line (if applicable) is essential in determining long term stability for those benchmarks constructed with Earth anchoring (fig. 11). Benchmarks that are to be occupied using GNSS need to have a minimal amount of surrounding obstruction in the hemisphere of applicable sky. An attempt should be made to establish benchmarks where obstructions such as buildings, overhangs, terrain, trees, fences, utility poles, or overhead lines exist below a 10 degree plane above the horizon. As a rule of thumb, it is much better to establish a new, completely open sky view site for a GNSS observation than to try to occupy an existing, reliable, wellknown benchmark with a somewhat obscured sky view (Henning, 2010).

\section{Software}

Software used for GNSS processing is complex with a variety of tools to process data. Most software programs provide the following basic utilities:

- Data transfer--Allows the transfer of files from the GNSS receiver or data collector to a personal computer (PC) processing package. Conversely, files can be transferred from the computer to external devices.

- Receiver INdependent EXchange (RINEX) format conversion.-Converts proprietary formatted GNSS data to a universal format known as RINEX; this conversion creates a raw observation file with station and antenna information, a navigation file containing GNSS orbits, and a meteorological file containing pressure, temperature, and relative humidity (Trimble, 1992-2002). These files are used for post-processing GNSS solutions.

- Data collector file editor--Allows the correction of field-entered data to be added into the data collector.

- Feature and attribute editor-Allows the management of processing geographic information systems (GIS) data collection. 

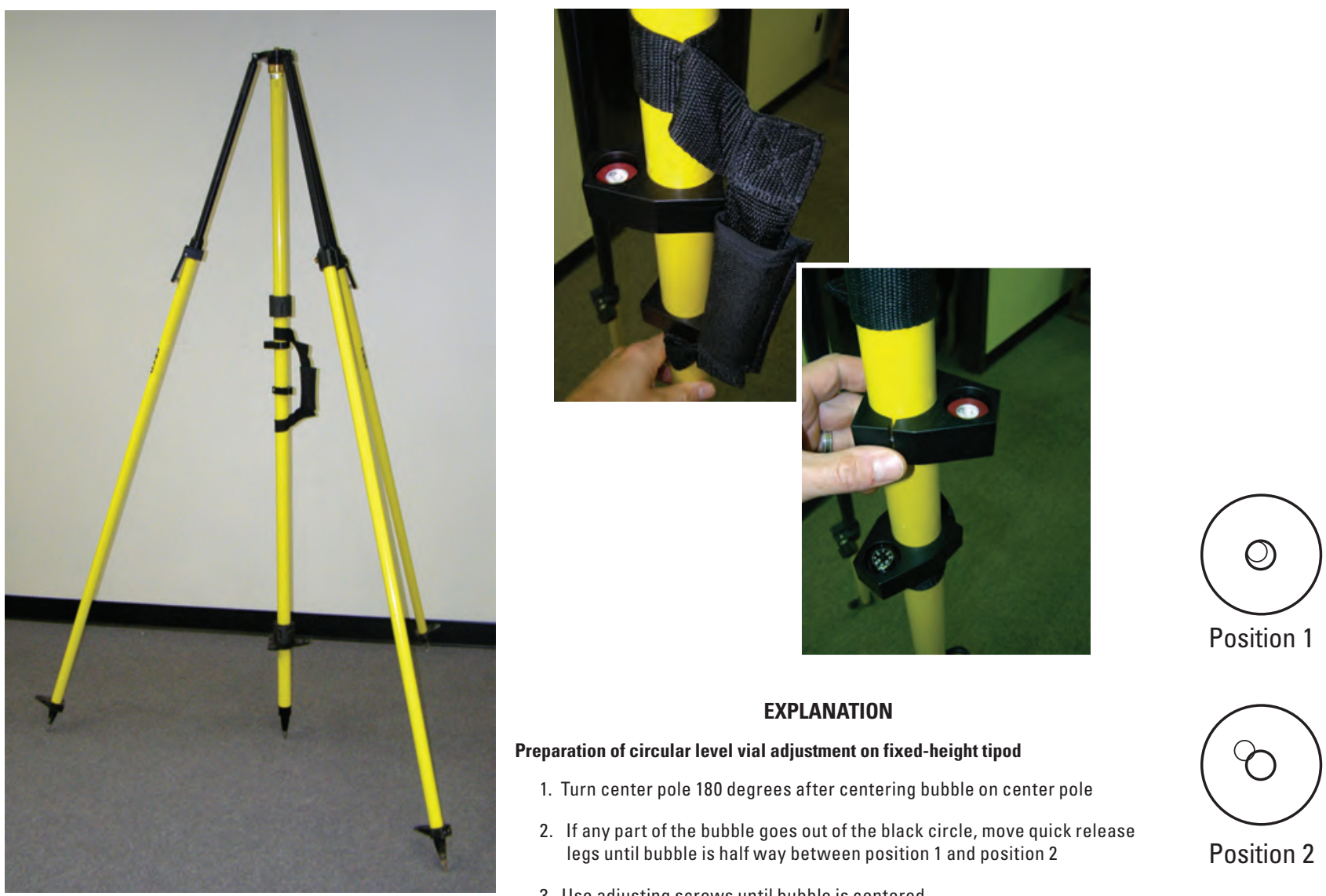

\section{EXPLANATION}

Preparation of circular level vial adjustment on fixed-height tipod

1. Turn center pole 180 degrees after centering bubble on center pole

2. If any part of the bubble goes out of the black circle, move quick release legs until bubble is half way between position 1 and position 2

3. Use adjusting screws until bubble is centered
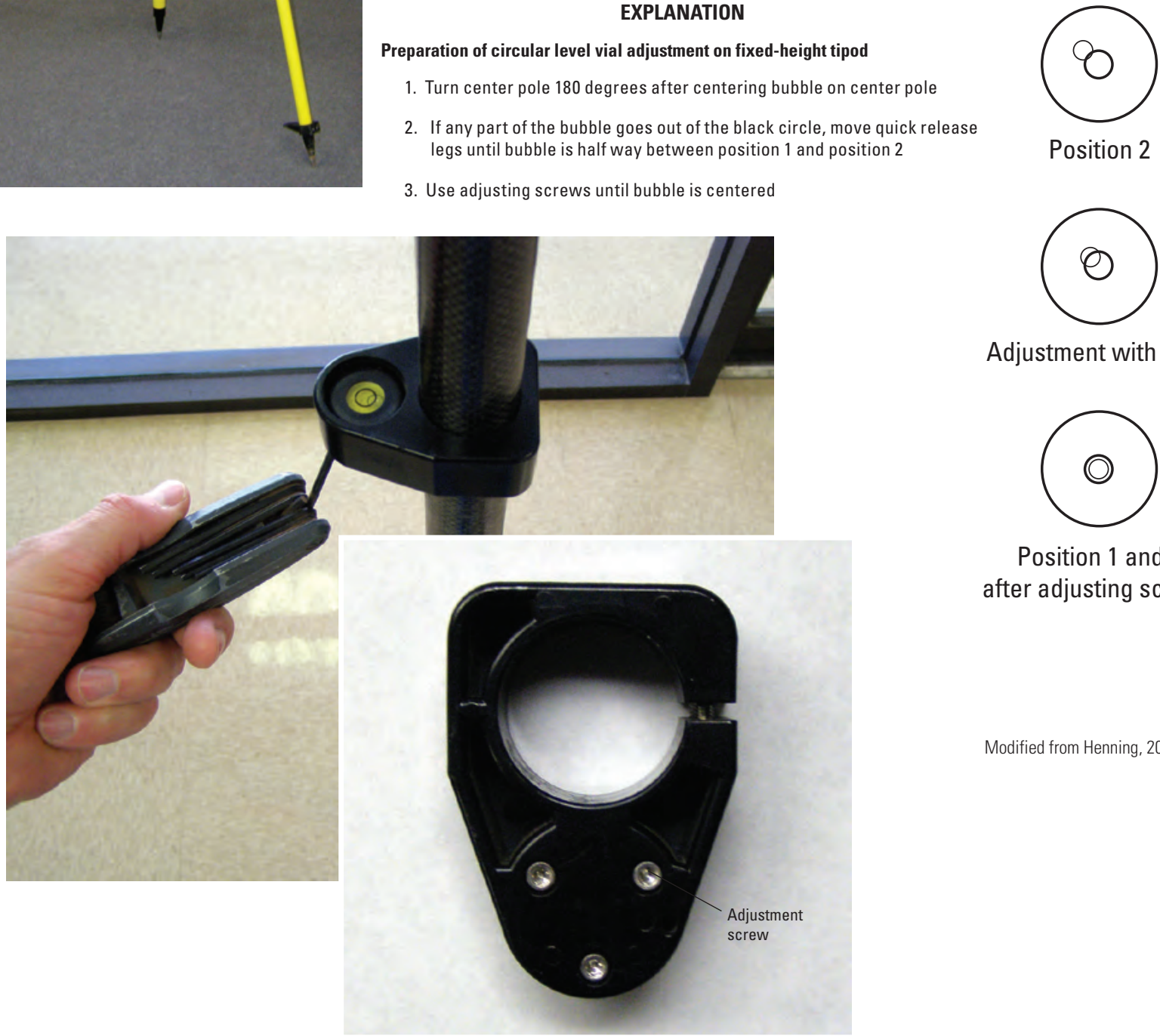

Position 2

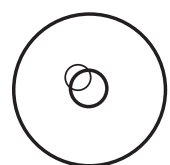

Adjustment with legs

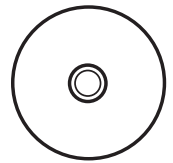

Position 1 and 2 after adjusting screws

Modified from Henning, 2010

Figure 9. Circular level vial adjustment on fixed-height center poles. 


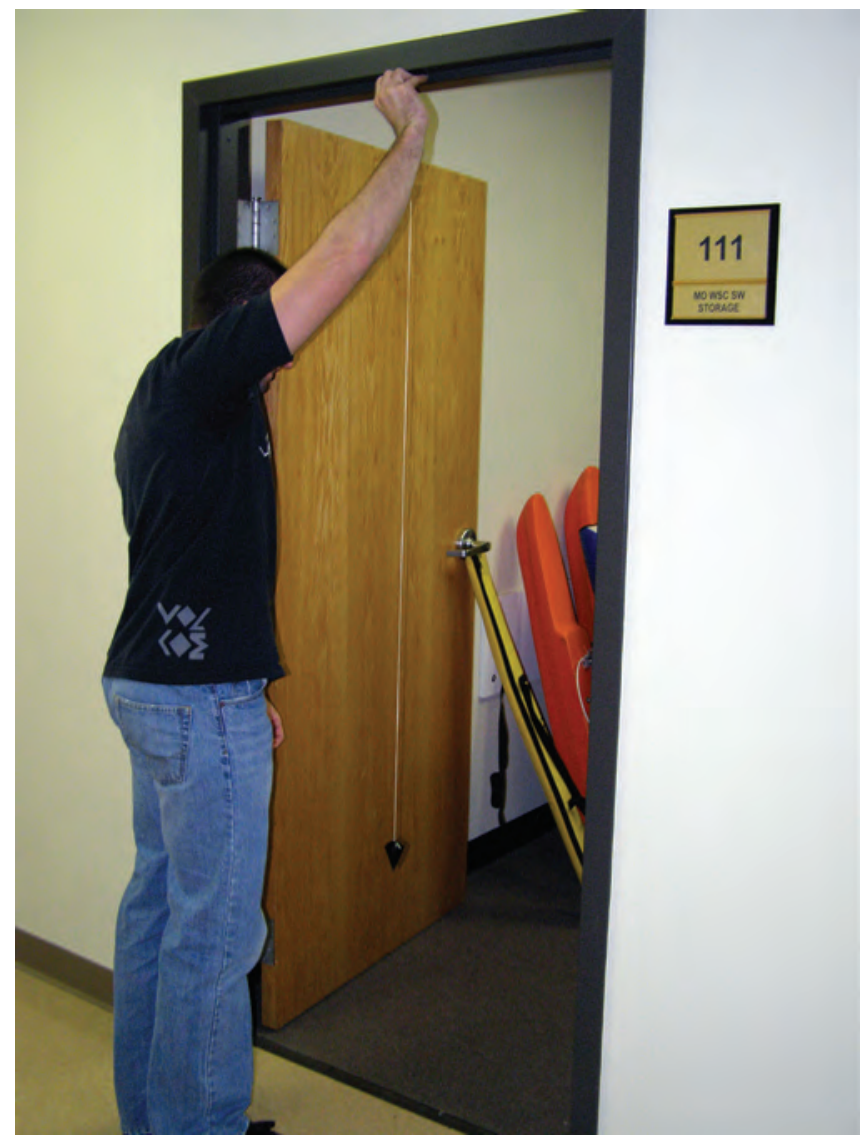

Using a plumb bob to ensure a vertical plumb line in a door jam.

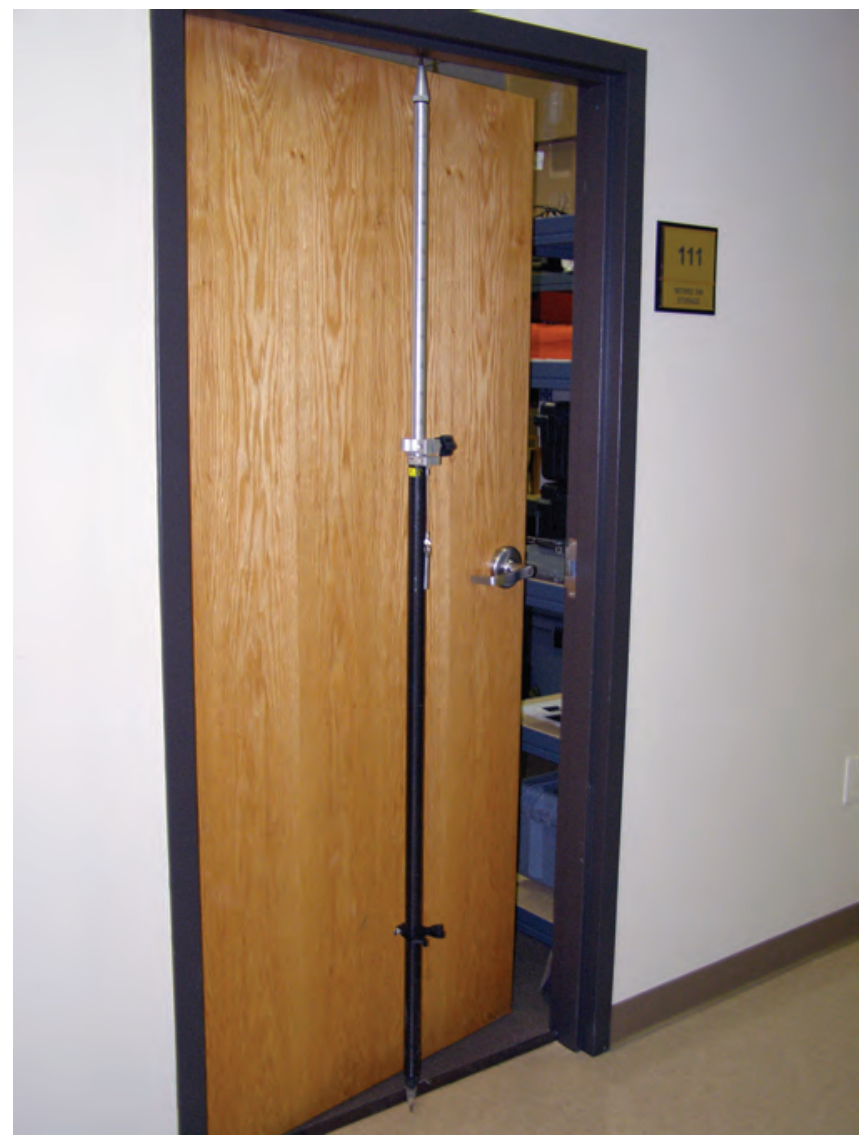

Establish shoe points on both ends of the adjustable fixed-height center pole and compress in the door jam.

\section{EXPLANATION}

Modified from Henning, 2010

\footnotetext{
Preparation of an adjustable fixed-height center pole used for a cicular level vial adjustment

1. Centerpunch a notch and establish an eyelet screw on the top of a door jam

2. String a plumb bob through the eyelet and mark a point (plumb) on the floor

3. Remove the adjustable fixed-height pole from the bipod legs and install a shoe point on both ends of the fixed-height pole

4. Set the bottom shoe point on the mark on the floor and adjust the adjustable fixed-height pole so the top shoe is compressed in the top jam

5. Proceed with the circular level vial adjustment
}

Figure 9. Circular level vial adjustment on fixed-height center poles.-Continued

- Mission planning tools.-Provides satellite information necessary for GNSS users to plan a GNSS campaign based on time, date, and geographic area.

- Grid manipulation tools.-Provides a mapping utility in which to extract geoid grids used to convert ellipsoid heights to orthometric heights. A grid over the conterminous United States uses a large amount of memory, which may not be available in some data collectors. An extraction of the geoid grid is usually performed for the area in which a GNSS campaign is led.
- Coordinate system manager--Allows access and editing of a geodetic database that contains coordinate systems, datum transformations, ellipsoids, and geoid models.

- Survey network adjustment.-A utility using a least squares adjustment among GNSS observations and known benchmarks.

Project software contains many additional functions, including the processing of GNSS baseline data, quality assurance closure reports, and digital terrain modeling and contouring. Additionally, many software programs have a built in form of computer automated drafting (CAD) that illustrates GNSS benchmarks, baselines, and measurements. 


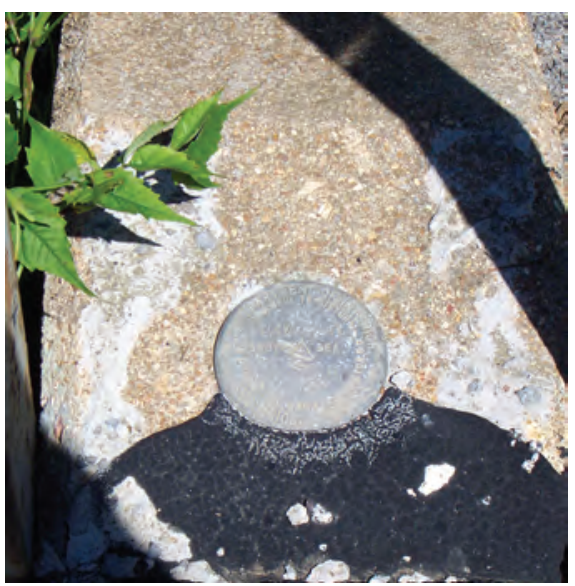

Monumented benchmark in top of concrete structure.

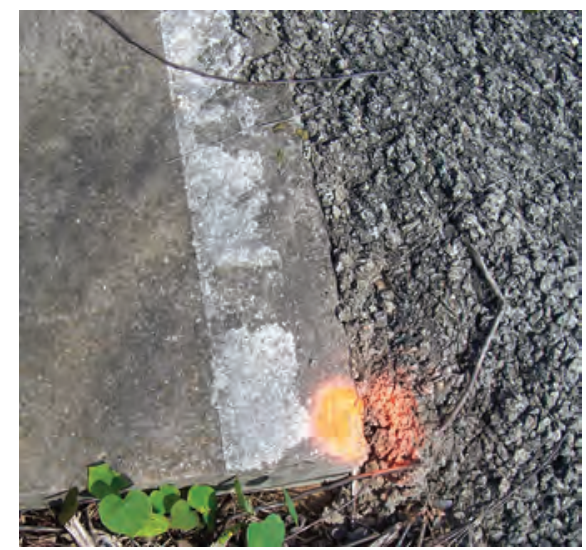

Non-monumented chiseled square benchmark in concrete structure.

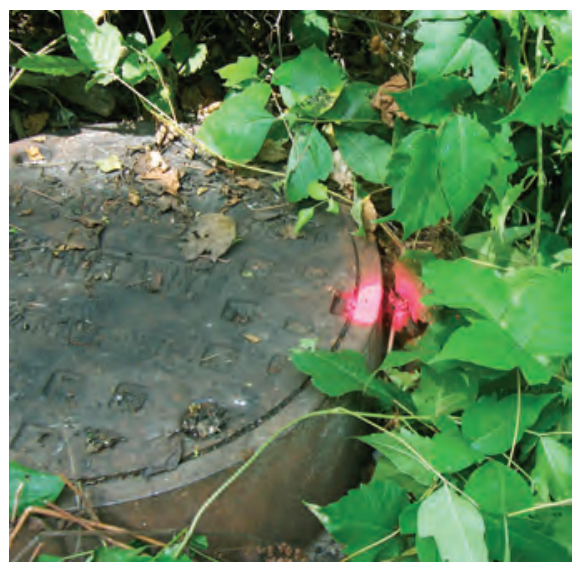

Non-monumented sawed groove benchmark in iron manhole structure.

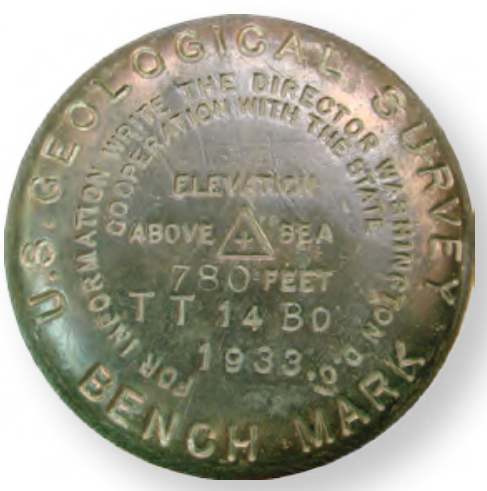

Example of U.S. Geological Survey benchmark historically used for topographic control.
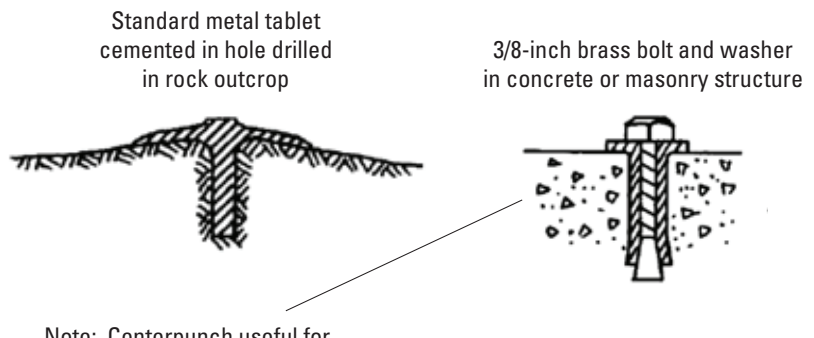

Note: Centerpunch useful for

constructing fixed-height pole points

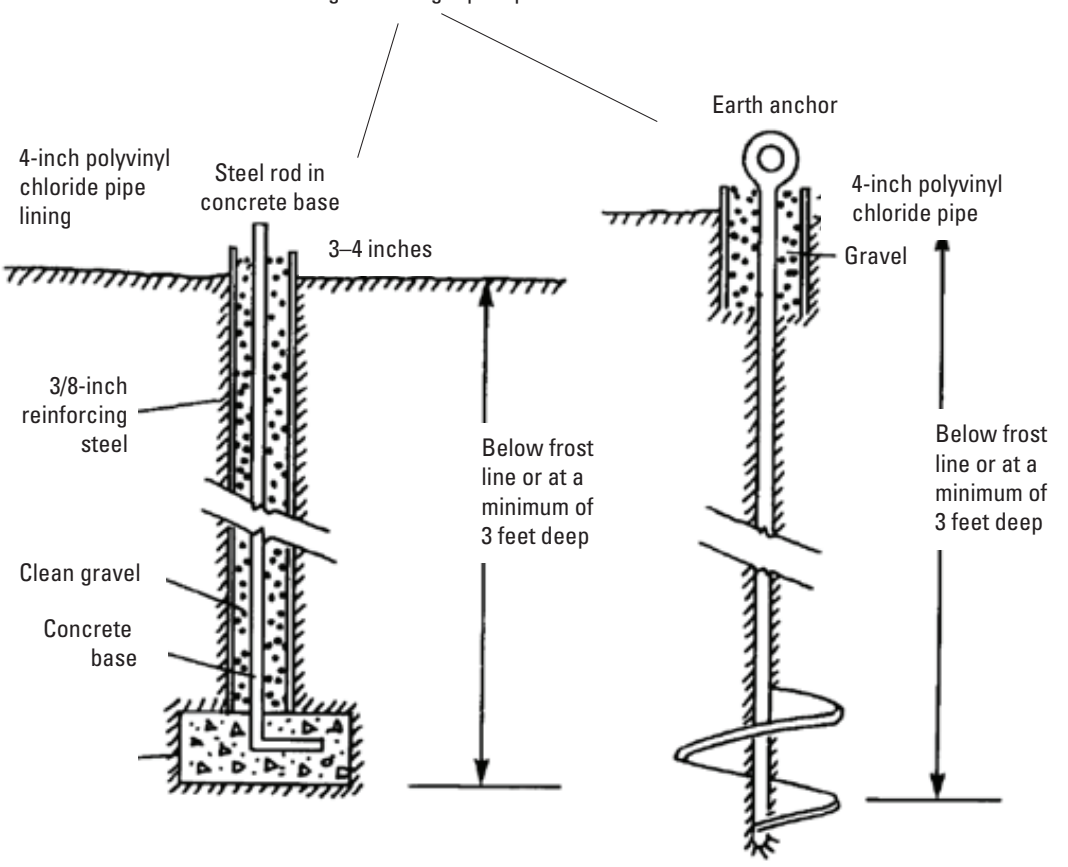

Modified from Kenney, 2010

Figure 10. Examples of monumented and non-monumented benchmarks and anchoring construction used for Global Navigation Satellite System (GNSS) observations. 


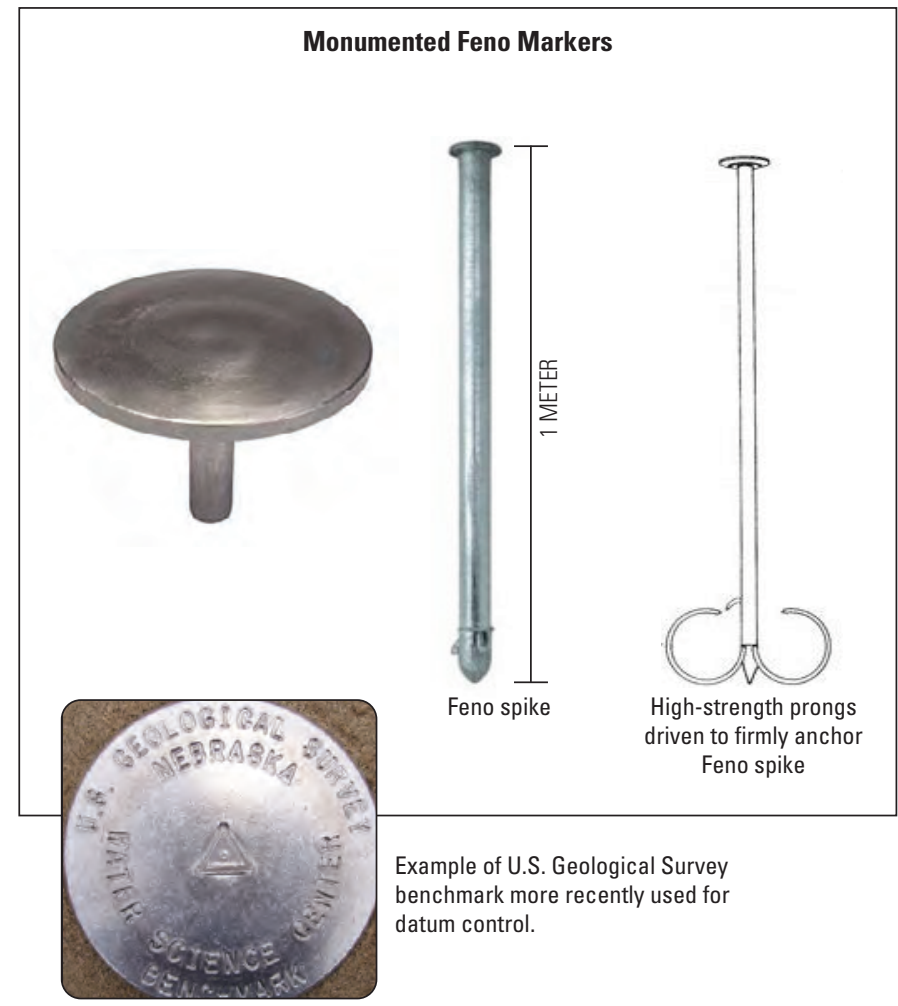

Figure 10. Examples of monumented and non-monumented benchmarks and anchoring construction used for Global Navigation Satellite System (GNSS) observations. - Continued

\section{GNSS Equipment Summary and Checklist}

Quality GNSS measurements are dependent upon equipment being in sufficient working condition. GNSS equipment should be thoroughly inspected before and after a survey campaign to ensure an adequate inventory and proper quality assurance. Table 3 provides an outline of the necessary equipment and quality-assurance recommendations for various GNSS campaign types.

\section{Mission Planning and Error Sources}

The concept of mission planning is crucial to all GNSS campaigns and is often overlooked, potentially compromising the quality of data as well as introducing delays in the process. For any GNSS campaign, a schedule of observation times should be evaluated with a logistics map that is used to assess observation locations and any localized baselines that may develop using single-base RTK or RTN surveys. A simple topographic map (often geo-referenced) is useful in illustrating open canopies for satellite reception of GNSS receivers, and the presence of contour lines can be used to evaluate radio communication potential for baselines. Online-acquired mapping utilities and satellite imagery are sufficient planning resources. A handheld GPS unit is quite useful for navigating to locations in the field.

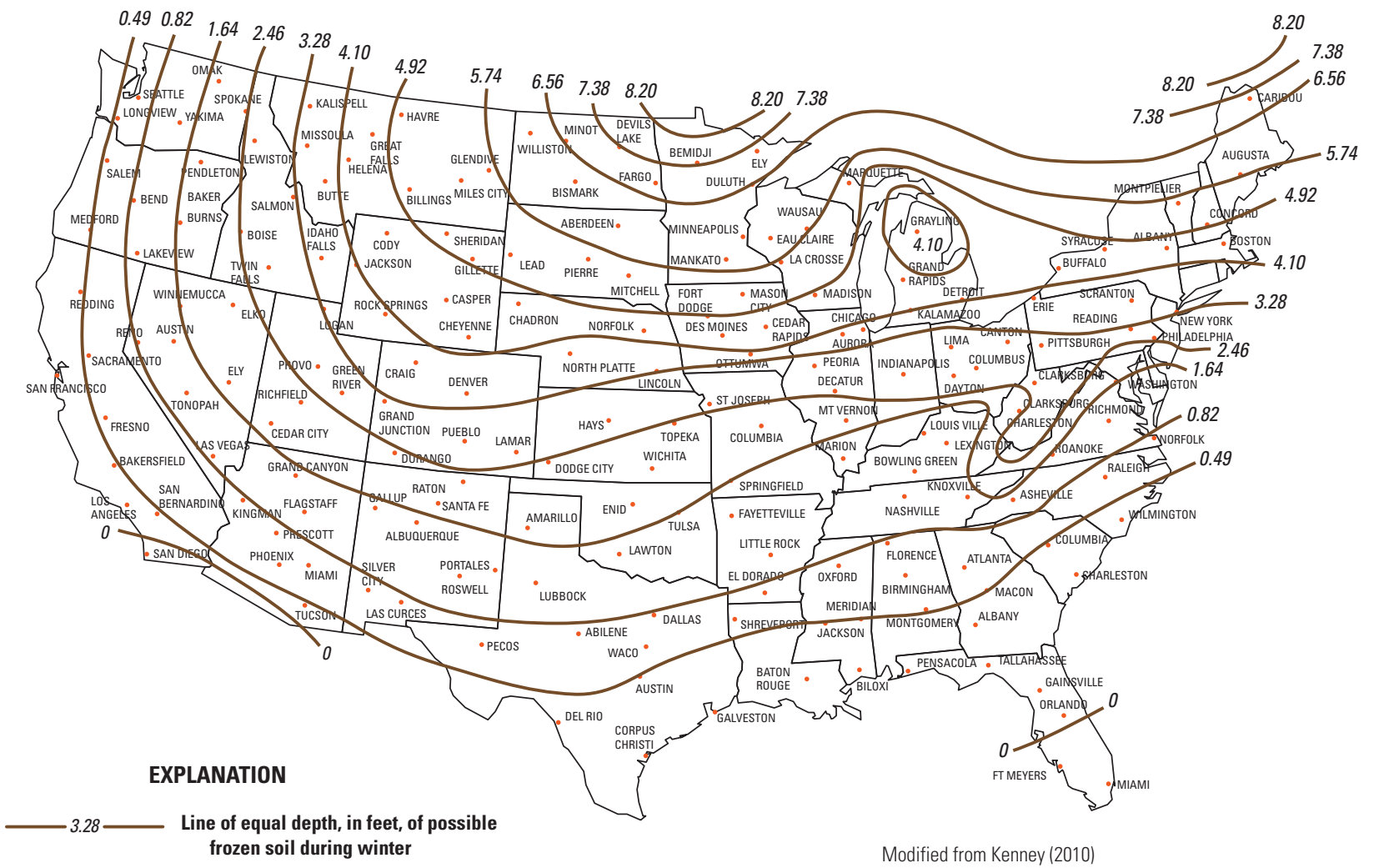

Figure 11. Depth of frozen ground for the lower 48 United States. 
Table 3. Global Navigation Satellite System (GNSS) equipment checklist and quality-assurance recommendations.

[GPS, Global Positioning System; GLONASS, GLObal NAvigation Satellite System; RTK, Real-Time Kinematic]

Post-processed GNSS-static

\begin{tabular}{lc}
\hline \multicolumn{1}{c}{ Equipment } & Quality assurance and accessories \\
\hline $\begin{array}{l}\text { Fixed-height GNSS receiver tripod* } \\
\text { GNSS receiver* }\end{array}$ & $\begin{array}{c}\text { Check height with tape, condition not warped or bent, sandbags for stability. } \\
\text { Dual frequency required, backup batteries, GPS+GLONASS+Galileo** } \\
\text { tracking preferred, latest firmware upgrades, minimize multipath design, } \\
\text { operating manual. }\end{array}$ \\
$\begin{array}{l}\text { Bluetooth or data cables checked, sufficient battery charge, backup batteries. } \\
\text { Bata collector }\end{array}$ & $\begin{array}{l}\text { Monumented or non-monumented considerations, anchoring construction } \\
\text { materials, datasheets with directions "to-reach" the benchmark location. }\end{array}$ \\
\hline
\end{tabular}

Real-time GNSS-single-base RTK

\begin{tabular}{cc}
\hline Equipment & Quality assurance and accessories
\end{tabular}

Fixed-height GNSS base receiver tripod

Fixed-height GNSS rover receiver bipod

Traditional tripod for GNSS broadcast radio and antenna mast

GNSS broadcast radio antenna

GNSS broadcast radio battery

GNSS rover receiver extended range antenna

GNSS base receiver

GNSS rover receiver

Benchmarks

\begin{tabular}{l} 
Equipment ${ }^{* * *}$ \\
\hline Fixed-height GNSS rover receiver bipod \\
GNSS rover receiver \\
Wireless modem with static internet protocol (IP) address \\
Benchmarks
\end{tabular}

Real-time GNSS-networks

Check height with tape, condition not warped or bent, sandbags for stability.

Check height with tape, condition not warped or bent.

Tribrach or flat plate for antenna mast; no loose legs.

Full-size whip antenna, tribrach or flat plate, cables, range pole.

Marine (hybrid deep cycle) battery for long occupations, backup battery.

Full-size whip antenna, mounting brackets, cables, range pole.

Dual frequency required, backup batteries, GPS+GLONASS+Galileo** tracking preferred, latest firmware upgrades, minimize multipath design, operating manual.

Dual frequency required, backup batteries, GPS+GLONASS+Galileo tracking preferred, latest firmware upgrades, minimize multipath design, operating manual.

Monumented or non-monumented considerations, anchoring construction materials, datasheets with directions "to-reach" the benchmark location.

Check height with tape, condition not warped or bent.

Dual frequency required, backup batteries, GPS+GLONASS+Galileo tracking preferred, latest firmware upgrades, minimize multipath design.

Assess broadband or cellular coverage area before campaign.

Monumented or non-monumented considerations, anchoring construction materials, datasheets with directions "to-reach" the benchmark location.

\footnotetext{
"Networks surveys involving GNSS would involve additional equipment.

** Global Positioning System (GPS) United States space-based satellite network combined with the GLObal NAvigation Satellite System (GLONASS) Russian space-based satellite network combined with the Galileo European space-based satellite network.

${ }^{* * *}$ All GNSS equipment noted for single-base RTK should be included as a backup for those instances where wireless modem coverage is not attainable.
}

\section{Benchmark Assessment}

For GNSS surveys concerned with quality assurance, benchmarks provide the most effective mechanism to tie the survey to local conditions and assess the quality of the survey; therefore, one mission planning necessity is the research of suitable benchmarks in the area of interest. The NGS datasheet retrieval (National Geodetic Survey Datasheet, 2010c) is a recommended first step in evaluating suitable benchmarks in the area and can be accessed online at: $h t t p: / / w w w$. ngs.noaa.gov/cgi-bin/datasheet.prl. This web site allows a radial, rectangular, and map search, as well as a search for benchmarks by USGS 1:24,000 scale topographic map, by county, and by benchmark attributes assigned by the NGS, such as permanent identifiers and station names (National Geodetic Survey Datasheet, 2010c). Another source that should be accessed by the web, researched, and contacted, is the list of identified state geodesy advisors: http://geodesy. noaa.gov/ADVISORS/AdvisorsIndex.shtml. State advisors, representatives, or interests may provide statewide temporary benchmarks established from geodetic leveling, or Federal or Cooperative Base Network upgrade information that may 
not be bluebooked [a term describing a formal submittal process of benchmarks into the NGS Integrated Database (IDB) maintained by the NGS], and published on the web as a NGS datasheet. As mentioned in the "Datum Establishment in the U.S. Geological Survey" section, the USGS originally established third-order benchmarks to aid in the development of topographic maps. Many of these marks are not published on the web; however, inquiries can be made at http://ask.usgs. gov to receive monumented and non-monumented benchmark information in the area of interest. Additional benchmark information may be recovered by other Federal, State, and local municipalities, such as the U.S. Army Corps of Engineers (USACE), Department of Transportation (DOT), and local municipal public works departments. All of these sources collectively can provide the largest benefit in locating benchmarks for planning a GNSS survey.

An evaluation of accuracy and reliability of all compiled benchmarks for the area of interest should be made. Datasheets from the NGS website described above may be selected based on the accuracy standard desired to support the GNSS campaign. There are many data types that are offered from the NGS that allow one to research benchmarks. These data types include vertical control order 1 and 2, GPS observations, tidal benchmarks, Height Modernization stations, and other stations associated with the Federal and Cooperative Base network and Airport Control stations. Accuracies of these various data types, including an expression of standard error, are offered by the NGS. When evaluating accuracies of local benchmarks published by the NGS, it is important to note the precision of the vertical elevation as described after the decimal place, independent of benchmark or data type. Regarding accuracy, an elevation of $634.38 \mathrm{ft}$ is considered more accurate than one expressed as $634.4 \mathrm{ft}$, or $634 \mathrm{ft}$. This is true for those marks published by the NGS; however, as mentioned earlier, there are other agencies and private entities that establish benchmarks that are not published, and should be scrutinized for acceptable accuracy. Generally, the NGS database serves as a clearinghouse for most of the benchmarks that have been established. In addition to accuracy, benchmark datasheets usually provide a date of the last recovery and the condition of the benchmark on that date, both of which are useful before heading to the field. Finally, reconnaissance of benchmarks should be part of mission planning that ensures recovery as well as suitability for satellite observations by identifying overhead or surrounding obstructions. Appendix 1 provides a good template for benchmark recovery and establishment documentation.

\section{CORS Assessment}

For GNSS campaigns involving post-processing or use of a RTN, GNSS users should examine the density of Continuously Operating Reference Stations (CORS) in the area of a campaign. CORS consist of a permanently installed GNSS receiver and receiver antenna where satellite positioning data are collected continuously. At a minimum, each CORS sends data to the NGS daily. As of October 2011, the CORS network contained more than 1,850 stations operated by 200 different organizations (W. Henning, National Geodetic Survey, written commun., 2011). CORS provide GNSS data that improve the precision of three-dimensional positioning relative to the NSRS. Data from the CORS network are distributed by the NGS and can be viewed at the following URL http://www. ngs.noaa.gov/CORS/. For planning purposes, it is important to map the distribution of CORS for static, RTN, and some single-base RTK applications. Generally, the greater the density and proximity of CORS to the area of a campaign, the more desirable. Essentially, CORS are information sources that a GNSS receiver uses to differentially correct its position [the difference in three-dimensional coordinates ( $\mathrm{x}, \mathrm{y}$, and $\mathrm{z}$ ) at the GNSS receiver is computed from the difference in observations at CORS]. This computation is done after observations are performed for post-processed methods, or in real-time as part of network modeling among a RTN. The closer the proximity of CORS to the receiver, the shorter the baseline distance, which generally decreases the amount of occupation time necessary to ensure quality positioning. When processing GNSS positions, space and terrestrial weather disturbances are more prominent concerns in areas void of CORS as opposed to areas that have a suitable density. Ideally, weather conditions would be homogenous within the area of the campaign, but disturbances that occur without suitable CORS would require longer observation times opposed to areas that are dense with CORS. Additionally, post-processed methods employ GNSS software that requires three or more baselines to process solutions; therefore, the greater the number of CORS, the greater the baseline availability to provide quality positioning. From a mission planning perspective, an area void of CORS stations, such as northwestern Kansas (fig. 12), requires longer observation times for longer baselines than areas with an increased density of CORS and resulting shorter baseline distances, such as central Missouri (fig. 12). Note that the CORS network is continually expanding as independently-owned CORS are contributed by other public and private organizations. The increase in Real-Time Networks, discussed later in the "RealTime GNSS" section, has also been a large contributor to the availability of CORS stations.

\section{lonosphere and Troposphere}

The ionosphere and troposphere are regions of the atmosphere that can potentially compromise the quality of GNSS positioning by delaying GNSS signals and corrupting radio communication. The ionosphere ranges from 30 to 600 miles above the Earth's surface and contains electrically charged particles. The ionosphere has a substantial affect on radio waves largely because of the total electron content (TEC), or total number of electrons present along a path between the satellite and GNSS receiver (Henning, 2010). The TEC varies according to solar and geomagnetic conditions at the time of 


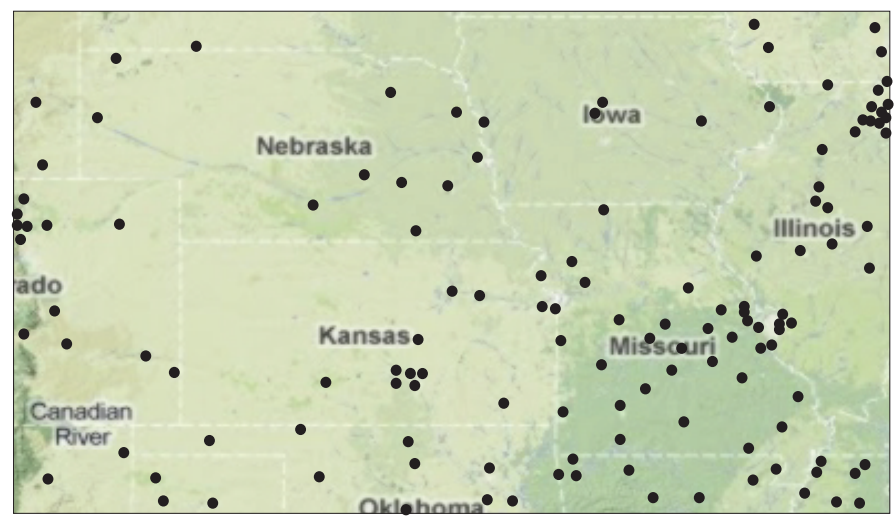

Modified from the National Geodetic Survey (NGS)-CORS http://www.ngs.noaa.gov/CORS

CORS webmaster update June 17, 2010

Accessed on September 14, 2010

EXPLANATION

- Continually Operating Reference Station

Figure 12. Density and proximity of Continually Operating Reference Station (CORS) managed by the National Geodetic Survey (NGS) in the Midwest. the day, geographic location, and season (Henning, 2010). Strong magnetic fields, known as sun spots, are common indicators of solar activity and to some degree, can be predicted in 11 year cycles (Henning, 2010). The official prediction peak of the next sun spot intensity (named solar cycle 24) will occur around the year 2014 (fig. 13) and is estimated to be below average in intensity (National Weather Service, 2009). During this peak, GNSS users can expect loss of satellite communications, loss of wireless connections and radio blackouts, and the inability for GNSS receivers to initialize (Henning, 2010).

The Space Weather Prediction Center (SWPC), operated by the National Oceanic and Atmospheric Administration's (NOAA's) National Weather Service (NWS), categorizes and reports three types of ionospheric disturbances with associated weather scales: Geomagnetic storms, Solar Radiation storms, and radio blackouts. Each of these are well documented and can be accessed on-line at: http://www.swpc.noaa.gov/. For mission planning purposes, a report of "Solar and Geophysical Activity and the Geophysical Alert Message" are space weather forecasting products that provide information about solar terrestrial conditions (National Weather Service, 2005). These products and additional space weather data can be

\section{ISES Solar Cycle Sunspot Number Progression}

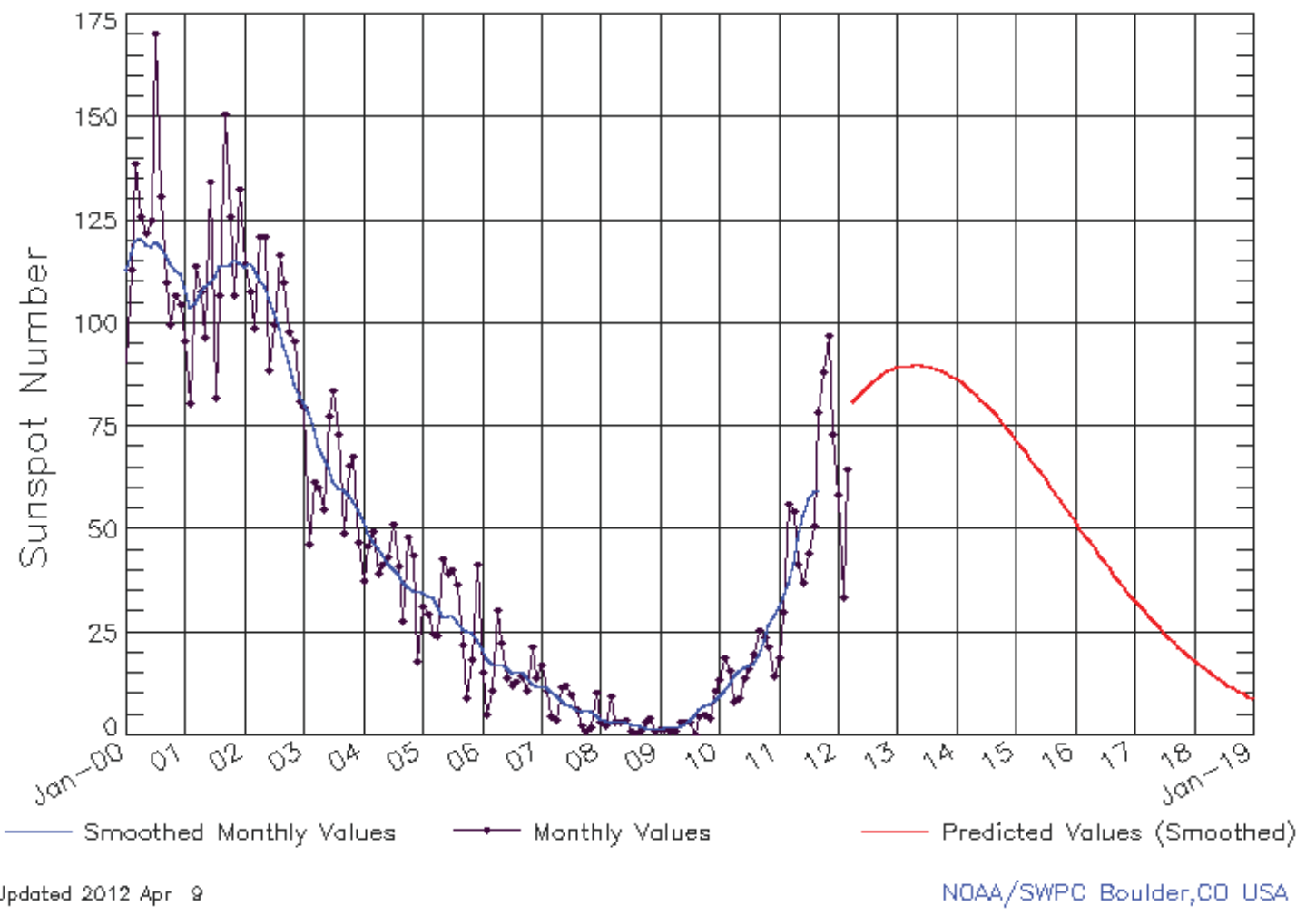

National Oceanic and Atmospheric Administration-National Weather Service Space Weather Prediction Center, Solar Cycle Progression, prediction update

Figure 13. Progression and forecast of ionospheric disturbance because of strong magnetic fields associated with sun spots. 
located at the aforementioned web site and may be disseminated by email subscription. In addition to space weather, the U.S. Coast Guard sends out a Notice Advisory to NAVSTAR Users (NANU), a message disseminated by email that provides the general health and forecasted outage for individual satellites. Satellite outages are issued approximately 3 days before a change in the operation of a GPS satellite, such as a change in orbit or scheduled on-board equipment maintenance (U.S. Department of Homeland Security, 2010).

The troposphere is another region of the atmosphere (extending approximately 30 miles from the Earth's surface) containing water vapor that produces our local weather. GNSS signal delays are more complex as a result of variable wet and dry conditions in the lower part of the troposphere. Most of the water vapor is contained within the lower 10 percent of the troposphere and is most difficult to model (ranging from 10 to 20 percent error) (Henning, 2010). Above 10 miles, the air is too cold and thin to contain much moisture, and thus represents the remaining dry part of the troposphere that can be accurately modeled within 1 percent error (Henning, 2010).

For mission planning purposes, it is important to maintain spatial weather consistency during any form of GNSS campaign, that is, avoid collecting observations where the base and rover are in different climatic conditions. Different climatic conditions include storm fronts, precipitation, temperature, and atmospheric pressure differences (Henning, 2010). Homogeneous climatic conditions are particularly important for single-base RTK surveys that do not have inherent mechanisms to interpolate conditions to the rover's site, such as a RTN.

GNSS signals that travel close to the horizon have the greatest distance of travel through the ionosphere and troposphere, which increase the atmospheric error previously described. To mitigate atmospheric error, an elevation mask should be set with each GNSS receiver to exclude signals near the horizon (fig. 14). An elevation mask of 10 to 15 degrees is desirable to mitigate these effects.

\section{Dilution of Precision}

The Position Dilution of Precision (PDOP) represents the geometry of the GNSS satellite constellation and its effect on precision. Satellite constellation geometry is the basis for a method, known as trilateration, which provides dimensions of position for the GNSS receiver. Regarding trilateration, the determination of point locations by distance measurements using geometry is much more favorable toward a well-dispersed satellite constellation over the field of view, as opposed to a less-dispersed satellite constellation that biases the field

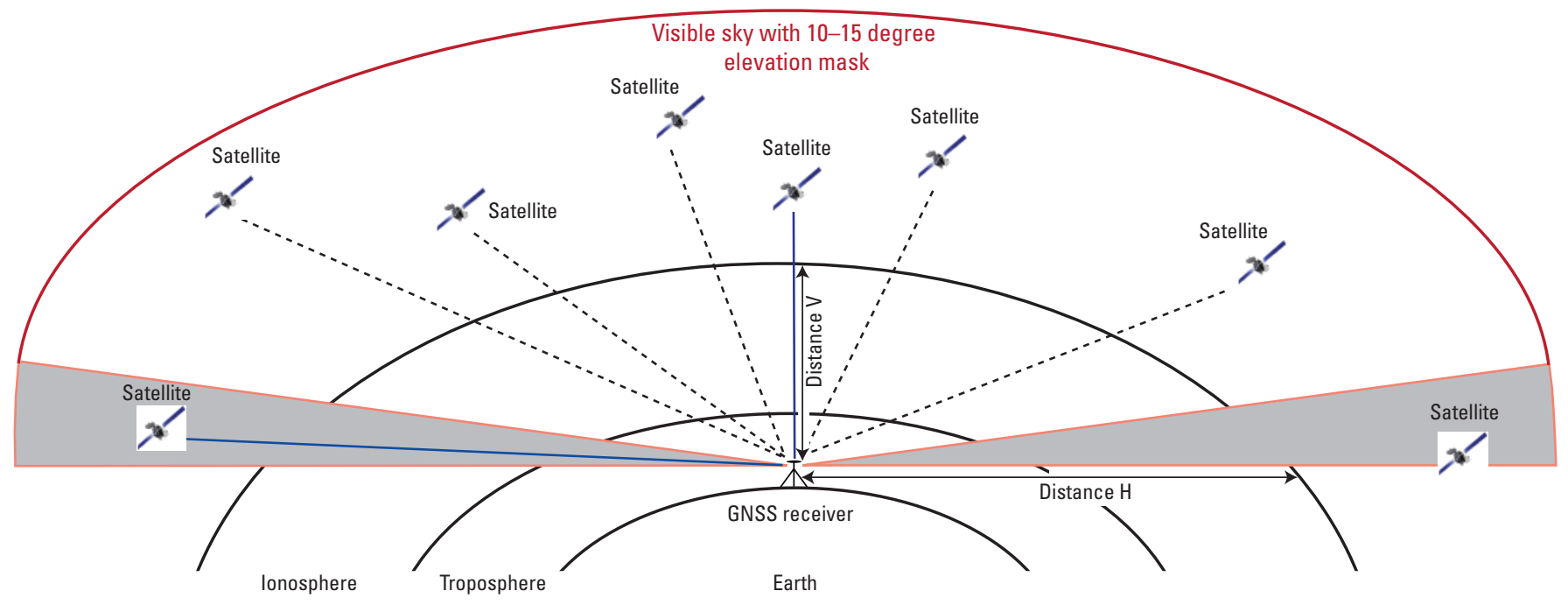

EXPLANATION

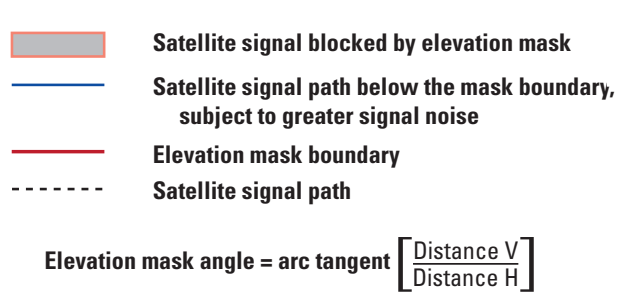

Figure 14. Elevation mask angle in relation to Global Navigation Satellite System (GNSS) signals traveling through the ionosphere and troposphere. 
of view; the satellites in the field of view of a GNSS receiver are positionally related to one another to provide a level of precision in each dimension of the receiver measurement. The configuration of these satellites affects horizontal and vertical uncertainties, and represents a unitless positioning value, known as PDOP, simplified and expressed as the ratio of the positioning accuracy to the measurement accuracy (Henning, 2010). A lower PDOP represents a well dispersed satellite constellation, which indicates a favorable ratio of positional accuracy to measurement accuracy. Conversely, geometry of the satellite constellation that is less dispersed produces a higher PDOP value. Additional dimensions of dilution of precision include horizontal, vertical, and time. The Horizontal Dilution of Precision (HDOP) represents horizontal accuracy in two dimensions, and the Vertical Dilution of Precision (VDOP) represents the vertical accuracy in one dimension (height). The relation between these variations and PDOP is expressed as: $\mathrm{PDOP}^{2}=\mathrm{HDOP}^{2}+\mathrm{VDOP}^{2}$ (Henning, 2010). The variations of PDOP and their relation to quality are illustrated in figure 15. The vertical component of the GNSS position is the most likely component to be lacking in quality if the PDOP values are high (Skeen, 2005).

A third variation, known as the Time Dilution of Precision (TDOP), represents how the satellite geometry is affecting the ability of the GNSS receiver to determine time. The association of time can be equated to a measure of the overall uncertainty in a GNSS solution, known as the Geometric Dilution of Precision (GDOP). GDOP is defined in a similar manner as PDOP, with the inclusion of time, and the relation between these two is defined as $\mathrm{GDOP}^{2}=\mathrm{PDOP}^{2}+\mathrm{TDOP}^{2}$.

General experience in GNSS positioning may broadly classify a PDOP value less than or equal to 3 as generally sufficient, a value greater than 3 but less than or equal to 5 as marginal, and values greater than or equal to 5 as poor. GNSS receivers may be set to mask and cutoff PDOP values of positioning, such that the receiver stops computing position fixes for a

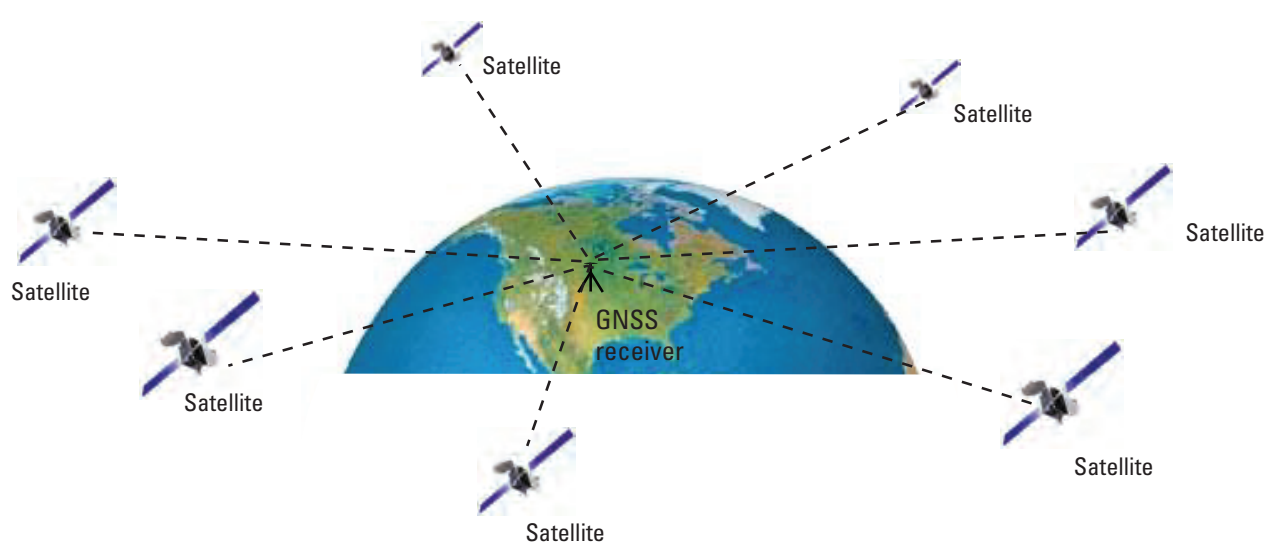

Poor vertical dilution of precision (VDOP)

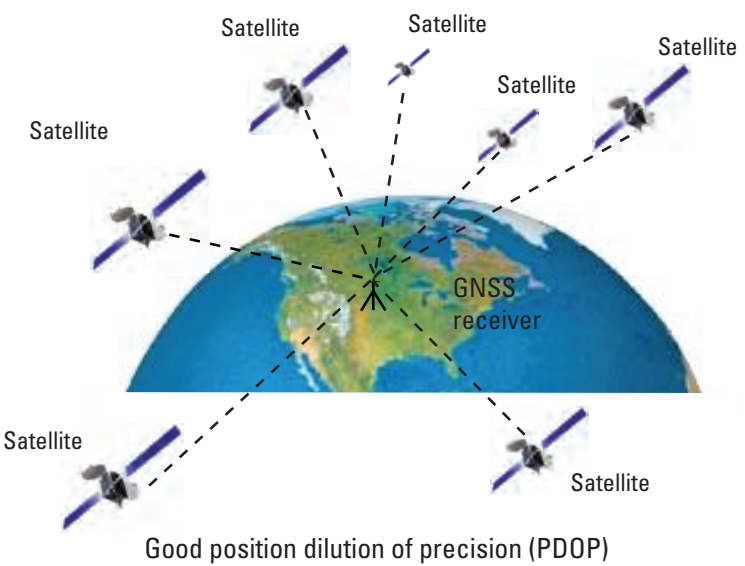

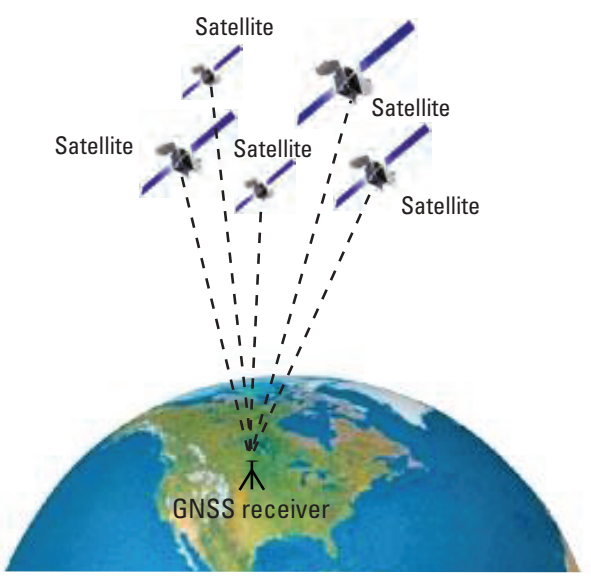

Poor horizontal dilution of precision (HDOP)

EXPLANATION

- - - - - - Satellite signal path

GNSS Global Navigation Satellite System

Figure 15. Horizontal and vertical relation to quality dilution of precision. 
satellite orientation that yields a PDOP greater than the mask value (Skeen, 2005). As discussed in the previous section, an appropriate elevation mask needs to be set to remove GNSS signals encountering lengthy travel through the ionosphere and troposphere; however, too much exclusion, such as a mask that is greater than 15 degrees, can introduce undesirable satellite geometry and adversely affect PDOP. Adding a supplemental fixed-height range pole to increase the height of the receiver may improve satellite availability and overall PDOP; however, the user needs to ensure that the extended height does not compromise the stability of the receiver.

\section{Multipath}

Multipath is another error source in GNSS surveying caused by surrounding objects that introduce a reflected signal, and thus a longer apparent distance of a GNSS signal to a receiver, rather than a distance representing a direct line of sight. Multipath can be caused by any objects along the signal path, but the most common are large buildings or structures, bridge superstructures, signboards, chain-link fences, and even mountains and water bodies. These objects reflect the radio waves, which distorts the process of trilateration by introducing a travel time that is too long or too short (fig. 16).

Reflected signals that occur near the receiver are more difficult to distinguish as opposed to those signals that have been reflected by objects further away because of the fact that the signal strength has not diminished as much as those reflected from objects further away. Most receivers that have been recently manufactured have algorithms with various degrees of immunity to secondary-path interference (Weill, 2003); however, there are several actions a user can take or practices a user can follow to combat multipath. First, the GNSS user should ensure a hemisphere of clear sky to provide the most effective possibility of minimizing multipath errors. Second, assurances should be made to make use of receivers that have internal ground planes to recognize flawed signals based on the angle of GNSS signals received. For

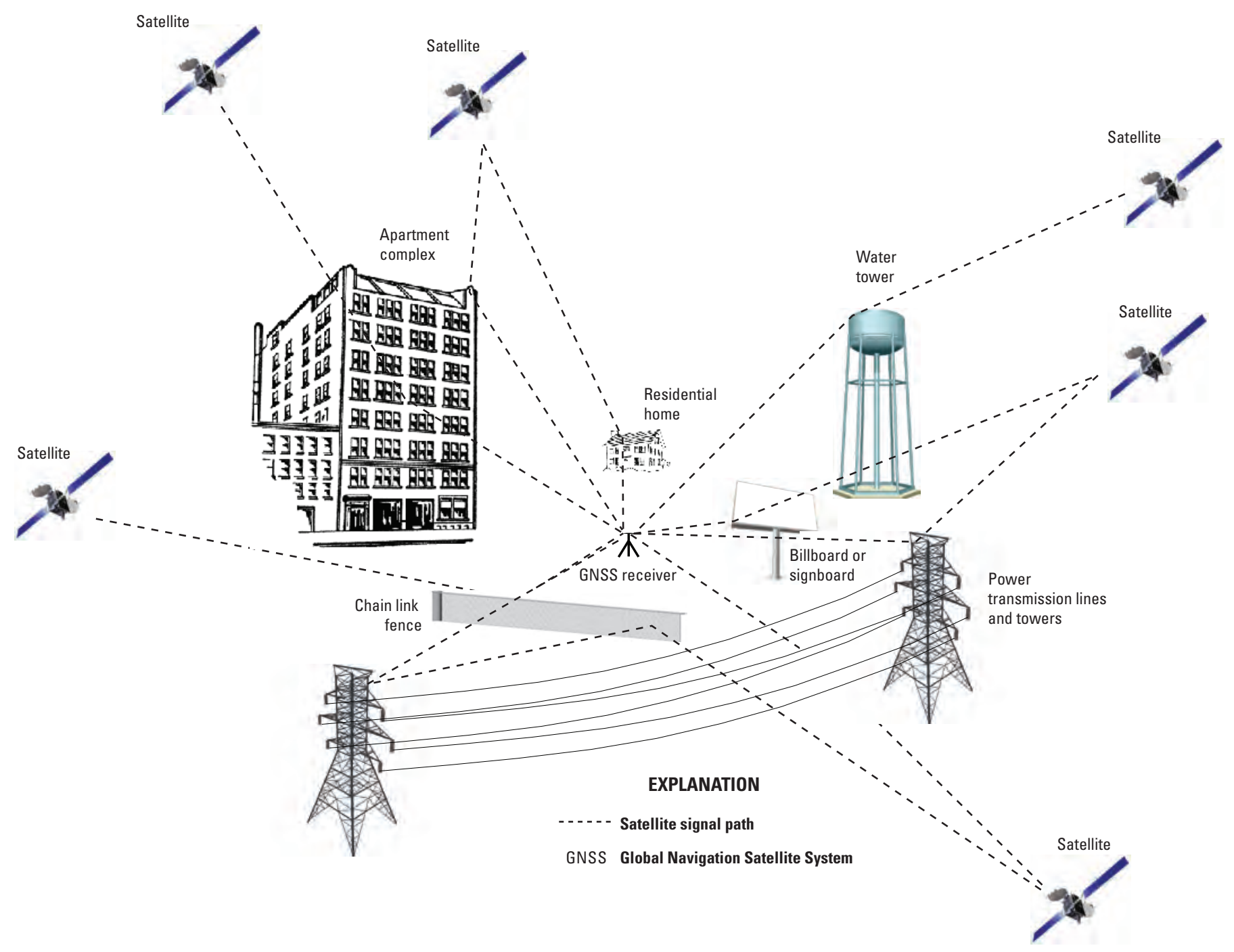

Figure 16. Multipath development from illustrated objects. 
those receivers that are collecting static data for many hours, secondary-path signals can be isolated by observing cyclic patterns in signal-to-noise ratios (SNR) (Weill, 2003). These static occupations use sophisticated software to model these cyclical patterns correctly during post processing (Henning, 2010). For real-time GNSS surveying, the typical occupation time occurs in seconds or minutes, which does not afford the opportunity to adequately model any present multipath condition (Henning, 2010). Redundant real-time observations with different satellite geometry can help mitigate multipath error (Henning, 2010).

\section{Summary of Error Source and Mitigation}

Assembling and powering up GNSS equipment are not the sole prerequisites to begin a campaign. Surveys supporting standardized data collection or interpretive investigations should be thought of in terms of accuracy, cost, and efficiency, all of which are interconnected and ensured by mission planning when scoping and budgeting such an effort. Table 4 provides error sources, general planning, and mitigation necessary to ensure a quality campaign. To further mitigate error sources and enhance GNSS positioning, redundancy should be exercised when possible. Redundancy may include multiple rover observations at benchmarks and objective points during a real-time GNSS campaign, or lengthy autonomous static observations separated by a length of time to ensure a change in atmospheric conditions and satellite geometry.

\section{Real-Time GNSS}

Traditional real-time positioning using GNSS is a widely accepted method of establishing a three-dimensional position that is perpetuated by a baseline vector from the modeled antenna phase center (APC) of a single fixed-base receiver to the modeled APC of a rover receiver (Henning, 2010). Current approaches use a real-time network of many reference stations that transmit corrections to the rover, enabling the computation of precise single- or multiple-baseline solutions (Jones and
Kelly, 2007). Both approaches involve real-time corrections that generate centimeter-level accuracy.

\section{Single-Base RTK}

A RTK survey continues to be the most used form of GNSS surveying. The real-time aspect to acquiring centimeter-level positioning provides efficiency and reduces costs. Single-base RTK surveys have been used to provide positioning for a variety of surveys, such as topographic, boundary, geodetic, engineering, and hydrographic. Single-base RTK surveys have also been used to monitor geotechnical and structural movement.

\section{Approach}

The single-base RTK surveying approach is the most commonly used approach within the USGS because this approach has many variations suited to many applications. The most common variation of the single-base RTK approach consist of a base station composed of a dual-frequency receiver mounted on a fixed-height tripod directly over a known benchmark. The base station includes a broadcast radio and long whip antenna, which is used to broadcast data corrections from the base receiver to a rover receiver. The rover receiver is used in a kinematic fashion, receiving data corrections from the base in "real-time" to derive elevation of an objective point. Before those corrections can be received, the rover receiver goes through an initialization process that downloads satellite almanac data, ephemeris data, time delay transmission from each satellite used to calculate distances to determine the receiver's position, and current date and time of each satellite. The almanac is composed of the general health and approximate positions of all the satellites in orbit. Satellite ephemeris data contains the precise position of the satellites.

This single-base approach typically ensures centimeterlevel positional accuracy for distances ranging to as much as 5 miles (or more depending on channel interference), but is largely dependent upon broadcast radio power and uninterrupted radio signal. A weak battery can limit communication

Table 4. Global Navigation Satellite System (GNSS) error source summary and mitigation.

[CORS, Continually Operating Reference Station; PDOP, Position Dilution of Precision]

\begin{tabular}{lll}
\hline Error source & \multicolumn{1}{c}{ Planning } & Mitigation \\
\hline Inefficiency & Logistics map & $\begin{array}{c}\text { Evaluate anticipated observations based on land-use topography, acceptable benchmarks, } \\
\text { density and proximity of CORS, and satellite availability. }\end{array}$ \\
$\begin{array}{lll}\text { Ionosphere } \\
\text { Troposphere }\end{array}$ & $\begin{array}{l}\text { Space weather forecast } \\
\text { Terrestrial weather forecast }\end{array}$ & $\begin{array}{c}\text { Evsure homogeneous atmospheric conditions within area of campaign, avoid and document } \\
\text { storm fronts, precipitation, temperature, and pressure differences. }\end{array}$ \\
Multipath & Obstruction diagram & $\begin{array}{c}\text { Avoidance of reflective objects such as large buildings, signboards, chain-link fences, water } \\
\text { towers, and power and transmission lines; elevation mask adjustment. }\end{array}$ \\
PDOP & Planning software & $\begin{array}{c}\text { Evaluate PDOP and satellite availability based on GNSS almanac for any given position and } \\
\text { obstruction condition. }\end{array}$ \\
\hline
\end{tabular}


range (Henning, 2010); therefore, it is important to ensure that the external battery used to power the broadcast radio supplies a minimum of 12 volts. The approach outlined above begins with a recovered benchmark, definition of a coordinate system and units, and $\mathrm{x}, \mathrm{y}$, and $\mathrm{z}$ coordinates of the recovered benchmark in that system. The base station receiver resides plumb over the known benchmark as the rover receiver is navigated to the objective point(s). Radio communication is important during single-base RTK work and should be evaluated logistically, as described earlier, during mission planning. Good survey practice is to ensure a direct line of radio communication without obstructions.

Another approach employs the single-base RTK system as a differential level. The base receiver location is established as an autonomous (unknown stand-alone) position at a location that is approximately half way between the known benchmark (thought of as the backsight) and the objective point (thought of as the foresight) (fig. 17). The half way distance is beneficial in equally reducing (thereby assuring) radio communication and baseline lengths to provide a more certain position. An effort should be made to establish this position at an elevation higher than the benchmark and objective point to ensure radio communication. A monumented or non-monumented benchmark should be established at the autonomous location, and the base receiver residing over the mark should acknowledge an autonomous position, as entered in the data collector. The data collector will display a mapping quality solution that typically has an expected positional accuracy of 3 to 10 meters (T. Bryant, Seiler Instrument, written commun., 2010.). For a simple transfer of elevation, there is no need to obligate the base receiver for a fixed amount of time to post-process a GNSS solution. The base will retain an autonomous, yet inaccurate, $\mathrm{x}, \mathrm{y}$, and $\mathrm{z}$ position, and the rover unit can be used to difference observed elevations at the known benchmark and objective point (fig. 17). Accuracy of the autonomously derived elevations are of no concern because the elevation (orthometric height) difference obtained between the known benchmark and objective point can be applied to the known elevation of the benchmark. The accuracy of horizontal positioning acquired while using this method is often suitable for surveys only concerned with elevation. This method essentially relies on the difference in ellipsoid heights between the stations, as well as a hybrid geoid model used in the data collector software to achieve the elevation of the unknown point(s). A redundant observation staggered by several hours should always be performed for important points. It is important to ensure the correct benchmark coordinates are applied to the differencing in this approach. Autonomously derived elevation differences possess certainty, but known coordinates, whether inaccurate or incorrectly entered, can compromise the entire approach.

The "differential level" approach using RTK has four distinct advantages:

1. Expedites the process of perpetuating elevation.

2. Does not obligate a base receiver over a known benchmark that may not have an open sky for satellite observations.

3. Provides an autonomous site selection void of obstruction for sufficient satellite observation.

4. Provides an autonomous site selection between known and objective points that reduce the baseline lengths (reducing the distance-weighted error inherent to the equipment manufacturer using RT methods) and assures better radio communication.

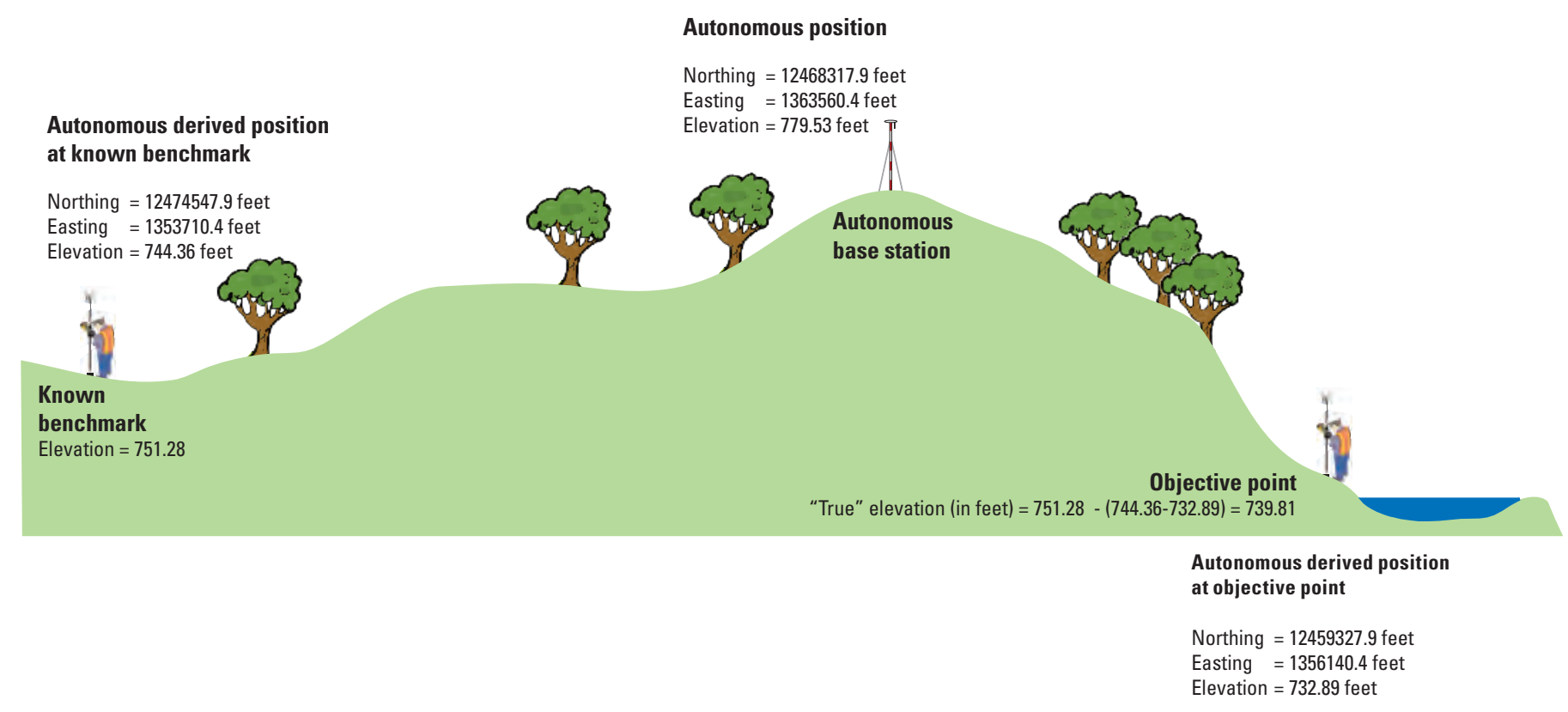

Figure 17. Example approach using single-base Real-Time Kinematic (RTK) as a level. 
This method may be considered to perpetuate vertical elevation; however, if horizontal coordinates are available at the known benchmark, a similar process of differencing autonomously derived horizontal positions between known benchmarks and objective points can be applied to yield horizontal coordinates at the objective point.

Another derivation of the "RTK as a level" approach is to simultaneously collect data at the base receiver while rover observations are performed. Provided mission planning assurances and unobstructed sky, data at the base receiver can be post-processed to produce coordinate values and provide another check during the survey to assure reproducibility. The disadvantage is the potential time constraint required to fulfill this approach. Static post-processing of GNSS data usually requires longer periods of data collection because sufficient data are required to resolve long baselines that exist between CORS and the survey base station; however, the growing number of CORS stations coupled with improved processing algorithms has made it possible to get sufficient coordinate estimates with as little as 15 minutes of static data (http://www. ngs.noaa.gov/OPUSI/Plots/Gmap/OPUSRS_sigmap.shtml).

In a purely autonomous fashion, any single-base RTK observation made from an autonomous base station can be differenced after the base receiver's position has been postprocessed; however, it is recommended that observations on known benchmarks occur within the effort to provide quality checks. The user may choose to post-process a solution at the base station or force a coordinate position by utilizing at least 3 , but preferably 4 , benchmarks (if available) that surround the area of interest. After successful initializations and subsequent single-base RTK observations have been made at each of the three benchmarks, a forced coordinate position or calibration can be made by keying in the proper coordinates from each point. Forced coordinate positions will propagate the correct coordinates to the base station, which can be accomplished in the data collector and is often referred to as a "site calibration." Note that the quality of the propagated position is only as good as the surrounding benchmarks. Site calibrations, also known as localizations, may continue further such that rover observations performed after a site calibration are now "localized" to the selected benchmarks. A localization will be discussed in greater detail later in this section.

\section{Quality Assurance}

Aside from equipment and mission planning assurances discussed earlier, single-base RTK surveys require some quality-assurance practices, including continuous broadcast radio communication, observation redundancy, and quality checks on benchmarks (there are circumstances when benchmarks with trusted accuracy do not exist and solutions may be postprocessed at the base station; however, it is recommended that a benchmark be used wherever possible to help validate the uncertainty of the effort). Intermittent or erratic radio communication leads to a degradation of positional accuracy possibly because of the latency of data reception (Henning, 2010). As users attempt to maximize the baseline limits of the singlebase RTK survey, assurances of radio communication can be enhanced by an extended range pole antenna at the rover unit or additional broadcast radios that can be set up as repeaters. To use broadcast radios as repeaters, base radios must be configured as specified by the manufacturer so that both radios are on the same frequency. Configurations of the base radios can be done on the front panel of some radios or by the use of software through a computer in other radios. It should be understood that longer single-base RTK baselines have a part per million (ppm) distance-weighted error that is coupled with a fixed error in the equipment. Typical single-base RTK equipment vertical accuracies assuming a minimum of 5 satellites and PDOP less than 4 are generally $2 \mathrm{~cm}+1 \mathrm{ppm}$ at the 68-percent confidence level. For example, a RTK observation using at least 5 satellites and PDOP less than 4 was performed with a 5-kilometer $(\mathrm{km})$ baseline. Regarding manufacturer specifications alone, the vertical accuracy may be expected to be:

$$
2 \mathrm{~cm}+500,000 \mathrm{~cm}\left[\frac{1}{1,000,000}\right]=2.5 \mathrm{~cm}
$$

For single-base RTK surveys, a fixed solution represents centimeter-level relative positioning and is generally obtained through differencing techniques (Henning, 2010). If the rover takes extended time to display a fixed position (beyond typical times observed for fixed positions), there could be underlying incomplete signal tracking, which would degrade accuracy sufficient for most survey single-base RTK applications. As a result, it is important to ensure the communication link is continuous, and the GNSS solution should become fixed in a 'normal' amount of time and should remain fixed for the duration of the data collection at the objective point (Henning, 2010).

Redundant observations should be a part of any singlebase RTK campaign. Observation redundancies with a staggered time less than an hour may be used to conduct a blunder check; however, for important points, staggered times of 3 to 4 hours between observations can provide a geometric change in the satellite constellation and atmospheric variability, thereby eliminating another source of bias. There are a variety of RTK survey types with different observation time requirements available within the data collector. Quality RTK observations require more observation time opposed to those that do not, such as simple topographic survey observations. Observations options such as an "observed control point" typically ensure collection of 180 epochs of data (usually 1-second data collection interval for 3 minutes). For quality GNSS surveys, the following blunder checks (or their observation equivalent) should be ensured during the single-base RTK survey.

Multipath \& General Blunder Check.-The user should walk away from the objective point a distance no less than 100 feet and approach the objective point from a different path for a second observation. This procedure provides the potential of a new multipath condition (whether multipath exists or not). 
Height Blunder Check.-GNSS signals $\mathrm{L}_{1}$ and $\mathrm{L}_{2}$ have wavelengths that are $19.03 \mathrm{~cm}(0.62 \mathrm{ft})$ and $24.42 \mathrm{~cm}(0.80 \mathrm{ft})$ in length (Kowoma.de, 2009). The user should physically adjust the rover range pole height by at least $0.80 \mathrm{ft}$ and input the new height into the collector. This adjustment provides an independent observation outside of the largest signal wavelength.

Reinitialization for Blunder Check.-The process of downloading satellite data should be reintroduced to provide some independence for the next observation. The user can simply invert the receiver on the rover pole until initialization has been lost, then position the pole in the upright position to regain initialization. Another option is to increase the elevation mask to lose initialization, then lower the mask to regain and measure.

Collectively, the GNSS user observes 180 epochs or 3 minutes of data, walks away from the objective point a minimum of 100 feet, inverts the rover receiver (or adjusts the elevation mask) to lose initialization, changes the rover rod height at least 0.80 feet and corrects the rover height entered in the data collector appropriately, walks a different approach path back to the objective point, then positions the rover receiver in an upright position over the objective point for the second observation. Both observations are averaged to produce the final result. This process is hereinafter referred to as a RT blunder check and should be recorded in the field notes to document that these redundant assurances are met.

For single-base RTK surveys in which a solution from the base receiver will be processed at a later date, or the base receiver resides over a known benchmark, it is recommended that the antenna height be measured before and after data logging. Inaccurate antenna heights are the most common error source with this work, so care must be exercised when the antenna height measurement is made. Measurements before and after data logging ensures another quality check regarding potential for systematic error.

Data collectors provide a precision, or repeatability of the solution as compared to the solution at the base station (usually indicated at the bottom of the data collector during singlebase RTK observations). These values can reflect precision at a 68-percent confidence level or "1 sigma." Values are often displayed as horizontal, vertical (orthometric), and root-meansquared (RMS) values resulting from the baseline solution (Henning, 2010). These values should be doubled to achieve the 95-percent confidence level or "2 sigma" confidence level. Check your GNSS manufacturer's settings to identify precision default settings. A blunder check and other quality assurance steps are further demonstrated in appendix 3 .

\section{Networks}

A real-time network (RTN) is a real-time positioning technique capable of operating over inter-receiver distances to as much as many miles with performance equivalent to a current single-base RTK system operating over much shorter baselines (Rizos, 2003). With a data modem or cellular phone link from the rover to the network of receivers, and the network's connection to the internet, precise positions can be logged consecutively, locally on the rover and to a geographic information systems (GIS) server in real time (Jones and Kelly, 2007). Essentially, these reference station receivers continuously stream data by way of the internet, a local area network (LAN), or a radio link to a centralized server. The server functions to monitor and model ionospheric, tropospheric, and satellite orbit and clock error, as well as other quality-assurance checks on raw data, storage of RINEX information, estimation of systematic error, and calculation and conversion of correction data to the end user in Radio Technical Commission for Maritime Services (RTCM), improved Compact Measurement Record (CMR+), or proprietary binary formats. More than 107 RTNs exist in the United States and many are continuing in the planning stages (W. Henning, National Geodetic Survey, written commun., 2011). RTNs provide benefit to many forms of infrastructure and are used actively by surveyors, machine control applications, construction, and precision agriculture. Table 5, derived from Henning (2009), identifies general benefits and drawbacks to the RTN. There are many interpolation methodologies within a RTN that have differing communications (either one-way or two-way) used to send position and correction data between the server and the rover unit (Henning, 2009). Regardless of the methodology used, the rover position is always the result of a differential baseline from a reference station (physical or virtual) whose coordinate is held fixed (Henning, 2009). The network itself maintains positional integrity through the use of RTN software and continual referencing to the NSRS; however, similar to the single-base RTK approach, the network provides solutions that can be constrained to local benchmarks to provide a localized adjustment that will optimize the precision of orthometric heights (fig. 18). As a stated drawback in table 5, local control (benchmarks) may not match the RTN solution. The difference between quality benchmarks and the network solution is likely minimal, but the user needs to consider and ensure what is held as "truth" for the GNSS campaign. The use of a benchmark in this regard provides:

1. A mechanism to evaluate the accuracy of the campaign (by differencing).

2. A localization to provide consistency among legacy geodetic work.

\section{Approach}

For quality GNSS work involving the establishment of vertical datum, the approach to real-time positioning in a RTN must include RT blunder checks, as described in the "Quality Assurance" section, on all local benchmarks and objective points. Exceptions to this statement are for topographic surveys or other efforts where the establishment of a quality 
Table 5. User benefits and drawbacks to Real-Time Network (RTN) Global Navigation Satellite Systems (GNSS).

[km, kilometer; mi, mile; NAD 83, North American Datum of 1983; ITRF, International Terrestrial Reference Frame; NSRS, National Spatial Reference System; OPUS, Online Position Users Service; NGS, National Geodetic Survey; TEQC, Toolkit for GPS/GLONASS/Galileo/SBAS Data; UNAVCO, University NAVSTAR Consortium]

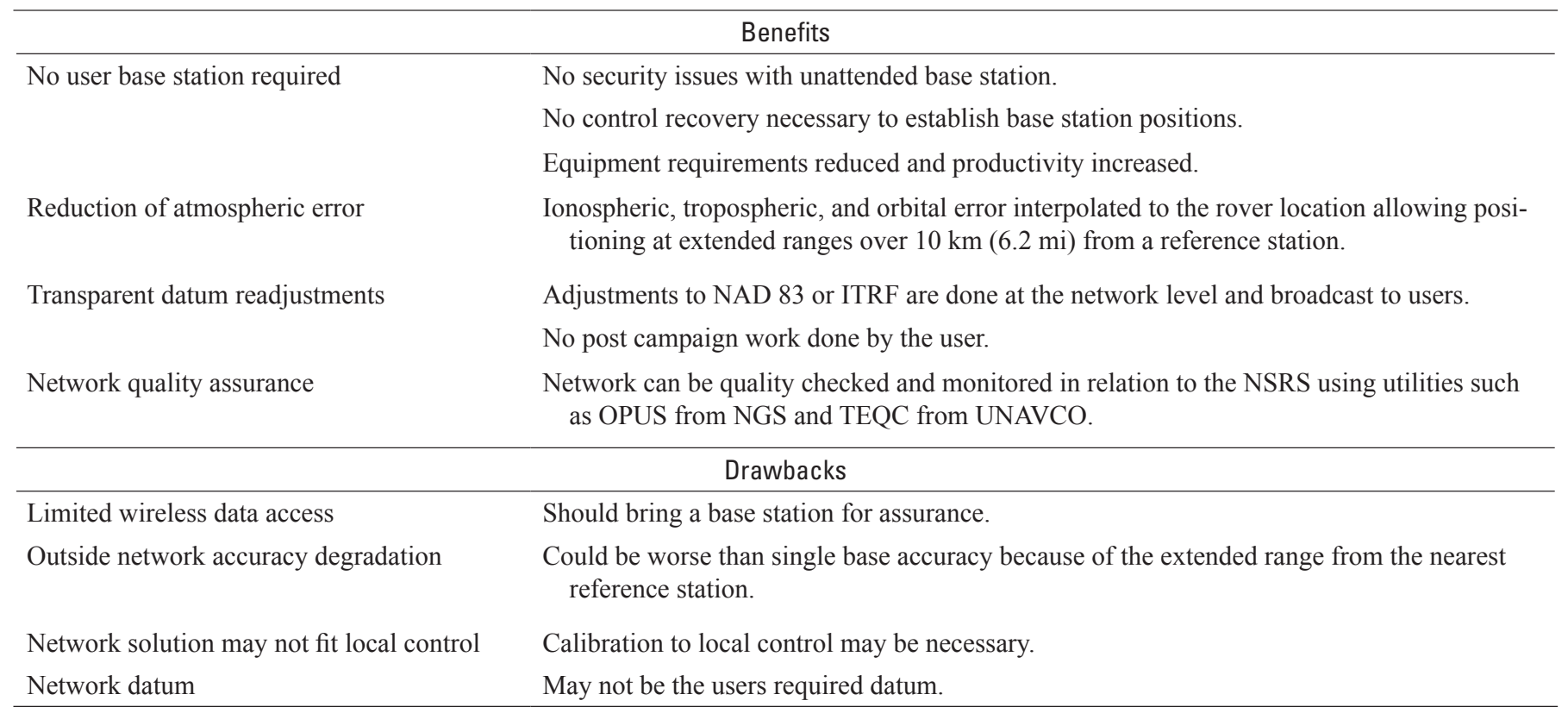

datum is not necessary. The user establishes a survey job and logs into the RTN system by cellular phone, compact wireless routers, or other internet communication. Areas void of cellular or wireless access may require an RTK bridge to propagate the data stream or simply require the use of a traditional base station to provide corrections to a rover. Depending upon the interpolation methodology within the network, communication may occur from the rover to a virtual base station (as an addition to the reference receiver network), from a rover to a reference (master) receiver (as part of the reference receiver network), or in a reverse manner from the rover, to the server, then back to the rover (Henning, 2009).

One of the more common interpolation methodologies involves the network creation of a virtual (non-physical) base station near the rover. The rover receives interpolated correction data and position for this virtual station, and corrections are computed to a new virtual base station once the user moves into a new area within the RTN (fig. 19). In most cases, positioning approaches described for single-base RTK can be applied within the network without the need of the base station.

Coordinates are typically the only output during positioning from RTNs. As a result, recording the following suggested metadata is useful for the user (from Henning, 2009):

Datum.-What are the datum, adjustment, and epoch used by the RTN? A comparison of positioning between the RTN and local benchmarks likely may vary because of a difference in a reference frame adjustment. As described earlier, the NAD 83 (NSRS2007) is an approximation of NAD 83 (CORS96). This realization can never be equivalent to the more rigorously defined NAD 83 (CORS96) in which CORS, OPUS, and many statewide RTN coordinates are distributed. The NAD 83 (2011), epoch 2010.0 will be considered the most recent adjustment beginning January 1, 2012 (National Geodetic Survey, 2012c).

NSRS Alignment.- - How was the network adjusted to CORS sites and what is the range of positional difference between reference station coordinates and those coordinates after adoption as a NGS CORS site? An example is provided in table 6.

Localization.-Was a project localization to benchmarks performed? If so, what benchmarks were held and what are the source, quality, and reliability of these as constrained points? What were the best fit residuals on these benchmarks?

Equipment.-What hardware (especially receiver antenna model), firmware, and software were used? What versions of the firmware were used in the data collector?

Guidelines and Standards.-What quality-assurance measures were adhered to?

Field Conditions. - What was the number of satellites observed, PDOP, local weather, space weather, RMS of the solution(s), horizontal and vertical precisions at 95-percent confidence?

Multipath Conditions. - Potential issues should be documented in a visibility diagram, including interference conditions such as power lines.

Communication.-Document resulting intermittent communications or interference, including vibration near transportation infrastructure such as railways or bridges, high tension wires nearby, or battery failure. 


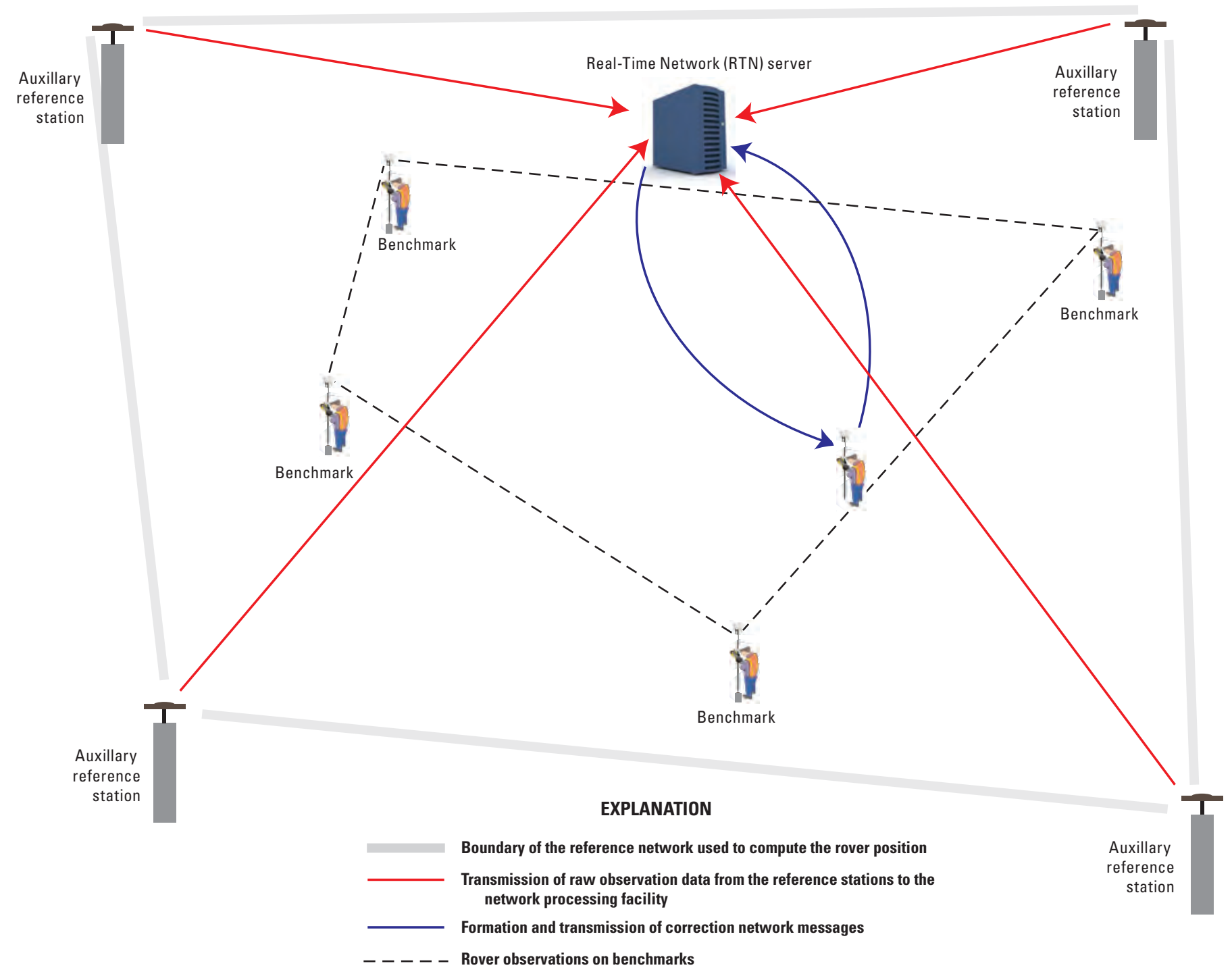

Figure 18. Real-Time Network positioning with a 4-point localization (site calibration) to known benchmarks.

\section{Quality Assurance}

Although a RTN contains quality checks and monitoring of the NSRS as well as atmospheric error modeling, quality positioning requires many additional considerations. As discussed earlier with single-base RTK positioning, the user should evaluate the precision of the position as reflected in the data collector. Empirical data offered by Henning (2009) states typical RTN precisions at the 95-percent confidence level are: horizontal $2-3 \mathrm{~cm}$, vertical (ellipsoid) $3-5 \mathrm{~cm}$, and orthometric heights 5-7 cm (using the current hybrid geoid model). The following quality assurances for RTNs are somewhat synonymous with a single-base RTK approach and are identified in table 7 (derived from Henning, 2009).

\section{Static GNSS}

Static GNSS surveying refers to collecting data by setting up a GNSS receiver and receiver antenna over a single point, and allowing measurements to be collected from positioning satellites for a time period. GNSS data collected during static surveys must be post processed against data collected from nearby control stations to correct atmospheric interference errors and produce survey-grade solutions. Static surveying techniques are used when high accuracy is required, such as establishing new control or reference marks. The most accurate results using GNSS technology are produced by static surveys. Static surveys often require more planning than other types of GNSS surveying. It is important to take note of antenna height above the survey mark, the type of receiver 


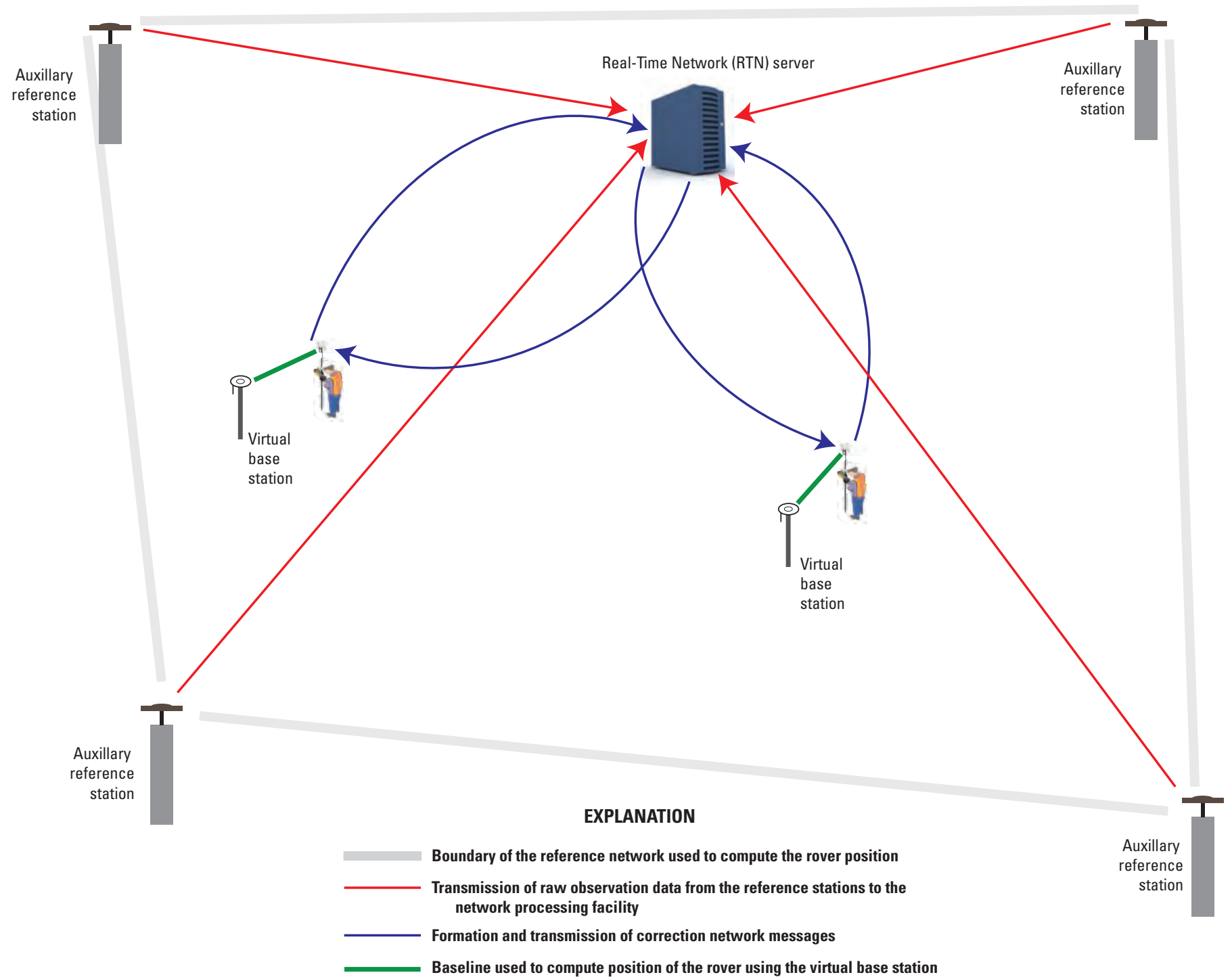

Figure 19. Real-Time Network positioning using the interpolation methodology of a virtual base station.

and receiver antenna being used, the start and stop time of each recording session, the recording interval, the description and location of the mark being surveyed, and the name of the surveyor. Having a standard form for surveyors to fill out during static data collection is a sufficient way to ensure all the necessary information is collected (see appendix 2 for a suggested form). There are two primary ways to process static data: through OPUS, or manually using baseline processing and network adjustment software. Both processing approaches have a direct correlation to the field collection method, time, and accuracy of the computed coordinates.

\section{Single Base: Online Positioning User Service (OPUS)}

OPUS is a simplified quality-assured service used to process static GNSS data, and is well supported by the NGS (www.ngs.noaa.gov/OPUS/about.html/). The NGS has provided
OPUS to process single-base GNSS data since 2002 (National Geodetic Survey, 2010c). The online service allows surveyors to collect static GNSS data at a known or unknown location, submit the data to NGS through an online interface, and receive quality processed positional coordinates through email minutes later. OPUS processes GNSS data using CORS, which are maintained by NGS and a wide variety of cooperating organizations. The user submits a static GNSS data set collected at a 1, $2,3,5,10,15$, or 30 second interval spanning from 15 minutes to 48 hours (midnight can only be recorded once). The user must also define the correct receiver antenna type that was used to collect the data, the receiver antenna height relative to the NGS defined antenna reference point (ARP), and a valid email address. OPUS accepts many file types that are automatically converted into RINEX (typically it is not necessary to convert your data file from its original format). If the specific file type cannot be converted by OPUS, all manufacturer software packages are capable of converting their files into RINEX format. 
Table 6. Example of Real-Time Network (RTN) reference station positioning change in Missouri after adoption into the National Geodetic Survey (NGS) Continually Operating Reference Station (CORS) network.

[MoDOT, Missouri Department of Transportation; m, meter; s, second]

\begin{tabular}{|c|c|c|c|c|c|c|c|c|c|}
\hline \multirow[b]{2}{*}{ Location } & \multicolumn{3}{|c|}{ NGS CORS position } & \multicolumn{3}{|c|}{ Current MoDOT position } & \multicolumn{3}{|c|}{ Change } \\
\hline & Latitude & Longitude & $\begin{array}{l}\text { Height } \\
\text { (m) }\end{array}$ & Latitude & Longitude & $\begin{array}{l}\text { Height } \\
\text { (m) }\end{array}$ & $\begin{array}{l}\text { Latitude } \\
\text { (s) }\end{array}$ & $\begin{array}{c}\text { Longitude } \\
\text { (s) }\end{array}$ & $\begin{array}{c}\text { Height } \\
\text { (m) }\end{array}$ \\
\hline MOAL_Albany & 401520.75427 & 941758.90487 & 254.866 & 401520.75411 & 941758.90481 & 254.885 & 0.0002 & 0.0001 & -0.0190 \\
\hline MOCE_Chilicothe & 394609.03952 & 933220.88533 & 183.997 & 394609.03943 & 933220.88519 & 184.021 & 0.0001 & 0.0001 & -0.0240 \\
\hline MOCD_Camdenton & 380209.39168 & 924623.11515 & 267.021 & 380209.39179 & 924623.11521 & 267.027 & -0.0001 & -0.0001 & -0.0060 \\
\hline MOCH_Charleston & 365505.22475 & 891907.58750 & 71.327 & 365505.22494 & 891907.58724 & 71.341 & -0.0002 & 0.0003 & -0.0140 \\
\hline MOHL_Hannibal & 394143.57203 & 912405.00590 & 169.999 & 394143.57208 & 912405.00573 & 170.016 & -0.0001 & 0.0002 & -0.0170 \\
\hline MOMK_Mokane & 384108.66048 & 915316.15264 & 170.868 & 384108.66044 & 915316.15242 & 170.886 & 0.0000 & 0.0002 & -0.0180 \\
\hline MOMV_Maryville & 402129.38595 & 945048.02244 & 278.928 & 402129.38579 & 945048.02238 & 278.952 & 0.0002 & 0.0001 & -0.0240 \\
\hline MOPA_Patton & 373107.60167 & 900044.07380 & 186.105 & 373107.60168 & 900044.07335 & 186.115 & -0.0000 & 0.0005 & -0.0100 \\
\hline MOWB_Warrensburg & 384852.19288 & 934428.61907 & 202.781 & 384852.19305 & 934428.61923 & 202.810 & -0.0002 & -0.0002 & -0.0290 \\
\hline MOBP_Blue Springs & 390032.80168 & 941726.15559 & 247.647 & 390032.80172 & 941726.15560 & 247.681 & -0.0000 & -0.0000 & -0.0340 \\
\hline MOSV_Savannah & 395715.14775 & 945051.31806 & 320.918 & 395715.14760 & 945051.31783 & 320.949 & 0.0001 & 0.0002 & -0.0310 \\
\hline MOTH_Thayer & 363338.80508 & 913311.75773 & 194.528 & 363338.80510 & 913311.75735 & 194.522 & -0.0000 & 0.0004 & 0.0060 \\
\hline MOWA_Wasola & 364731.02968 & 923449.74030 & 359.351 & 364731.02943 & 943449.74000 & 359.347 & 0.0002 & 0.0003 & 0.0040 \\
\hline MOBU_Buffalo & 373935.05486 & 930610.47544 & 306.576 & 373935.05478 & 930610.47531 & 306.599 & 0.0001 & 0.0001 & -0.0230 \\
\hline MOCL_Clinton & 382319.74363 & 934521.33626 & 208.083 & 382319.74359 & 934521.33628 & 208.120 & 0.0000 & -0.0000 & -0.0370 \\
\hline MODX_Dexter & 364824.82031 & 895842.95945 & 89.055 & 364824.82026 & 895842.95900 & 89.074 & 0.0000 & 0.0004 & -0.0190 \\
\hline MOED_Edina & 401111.65645 & 921030.28845 & 194.635 & 401111.65658 & 921030.28851 & 194.673 & -0.0001 & -0.0001 & -0.0380 \\
\hline MOHA_Harrisonville & 383633.08859 & 941912.88460 & 242.611 & 383633.08864 & 941912.88462 & 242.652 & -0.0000 & -0.0000 & -0.0410 \\
\hline MOMA_Manes & 372234.70262 & 922428.44110 & 373.789 & 372234.70253 & 922428.44081 & 373.799 & 0.0001 & 0.0003 & -0.0100 \\
\hline MOMG_Mountain Grove & 370751.66958 & 921839.95187 & 420.866 & 370751.66934 & 921839.95149 & 420.877 & 0.0002 & 0.0004 & -0.0110 \\
\hline MOMO_Monett & 365500.48625 & 935859.01644 & 381.756 & 365500.48615 & 935859.01626 & 381.769 & 0.0001 & 0.0002 & -0.0130 \\
\hline MONC_New Cambria & 394531.12304 & 924433.97236 & 220.903 & 394531.12313 & 924433.97234 & 220.935 & -0.0001 & 0.0000 & -0.0320 \\
\hline MONE_Nevada & 375156.71994 & 942058.36961 & 222.360 & 375156.71989 & 942058.36968 & 222.388 & 0.0001 & -0.0001 & -0.0280 \\
\hline MOOF_O'Fallon & 384514.06197 & 904144.78111 & 120.001 & 384514.06194 & 904144.78094 & 120.021 & 0.0000 & 0.0002 & -0.0200 \\
\hline MOSE_Seymour & 370920.77954 & 924514.32616 & 472.965 & 370920.77936 & 924514.32597 & 472.975 & 0.0002 & 0.0002 & -0.0100 \\
\hline MOSB_Shelbina & 394154.74930 & 920309.79903 & 204.313 & 394154.74930 & 920309.79898 & 204.343 & 0.0000 & 0.0000 & -0.0300 \\
\hline MOVB_Van Buren & 365736.45577 & 910337.81109 & 136.821 & 365736.45578 & 910337.81061 & 136.835 & -0.0000 & 0.0005 & -0.0140 \\
\hline MOWW_Warsaw & 381512.09657 & 932142.33168 & 192.844 & 381512.09653 & 932142.33165 & 192.873 & 0.0000 & 0.0000 & -0.0290 \\
\hline
\end{tabular}


Table 7. Real-time Global Navigation Satellite System (GNSS) quality assurances for single-base Real-Time Kinematic (RTK) and a Real-Time Network (RTN).

[ARP, antenna reference point; NA, not applicable; PDOP, Position Dilution of Precision; NOAA, National Oceanic Atmospheric Administration; SWPC, SpaceWeather Prediction Center; OPUS, Online Position Users Service; IP, internet protocol; RTCM, Radio Technical Commission for Maritime Services; CMR+, Compact Measurement Record; km, kilometer]

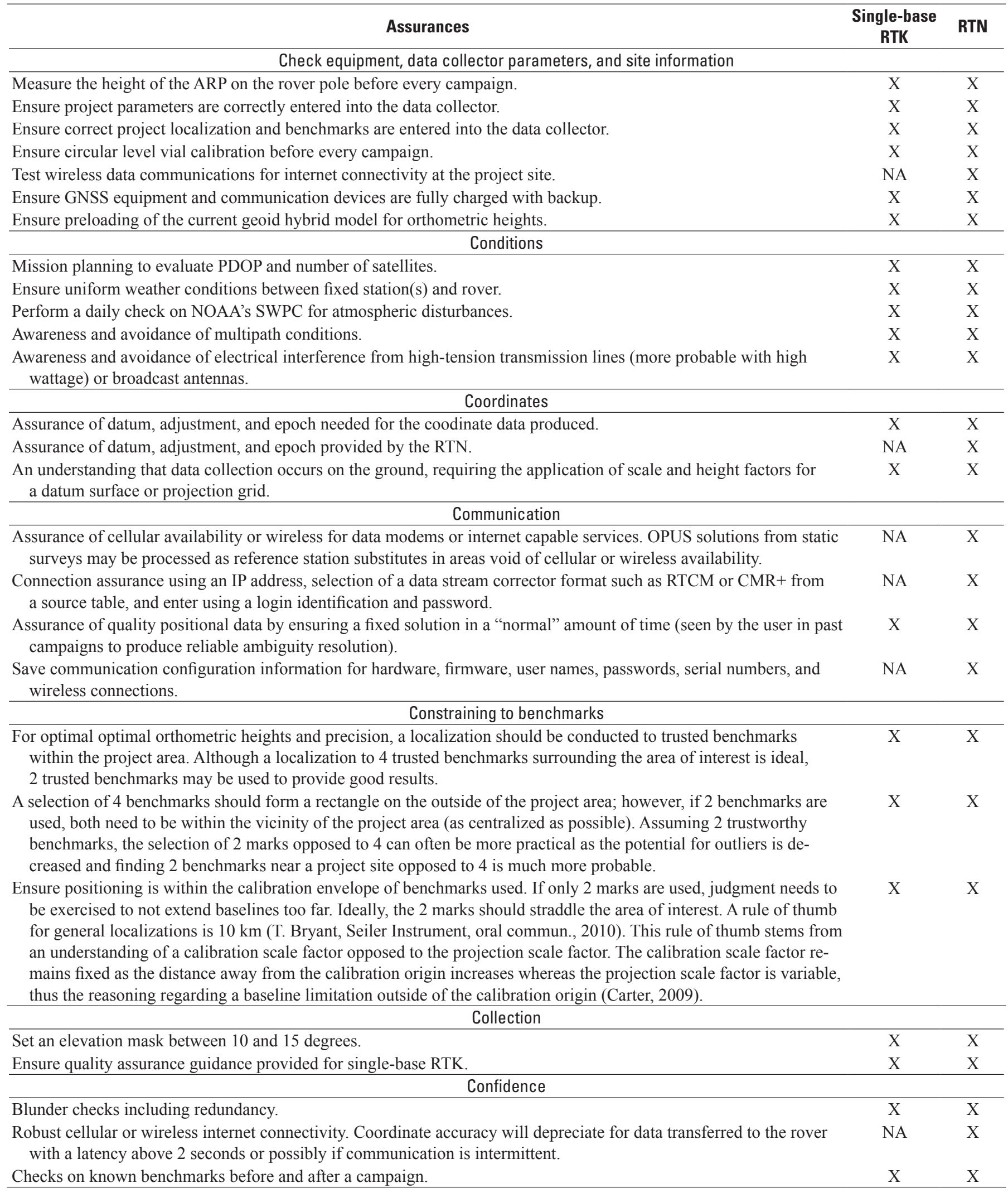


Traditional OPUS-Static (OPUS-S) processes the user's file against three CORS using the Program for the Adjustment of GPS Ephemerides (PAGES) software. PAGES is a NGS program that performs vector reduction to process GNSS baselines. PAGES processes the GNSS file using individual CORS and determines a position. The final coordinates reported are an average of the three CORS independent single-baseline solutions. Peak-to-peak errors are reported with the final solution. Peak-to-peak errors represent the difference between the maximum and the minimum value of the coordinates obtained from the three baseline solutions. In addition, the final report describes the percent of observations recorded in the data file that were used in processing, percentage of ambiguities fixed, and the overall RMS. The percent of observations used indicates how many of the recorded observations were of sufficient quality to include in the analysis. This number will be lower if the sky view at the benchmark was not clear, multipath errors were recorded in a number of the observations, there was movement of the tripod, or there were other error sources that might make the observations poor.

Detailed discussions regarding ambiguities are left to other references. It is important to understand that OPUS-S attempts to fix phase ambiguities to their integer value (fix integers), identifies the percentage of ambiguities that OPUS-S "thinks" it fixed correctly, and reports that as a percentage of all ambiguities (M. Schenewerk, National Geodetic Survey, written commun., 2011). The overall RMS represents the precision of a solution and it is calculated as the square root of the average mean squared error from the final coordinate to each of the single baseline coordinates. Because all processing of the GNSS data is performed through the OPUS website using CORS as control points, the user can benefit from time savings in a number of areas:

- No field trips for reconnaissance and "ground truthing" benchmarks.

- No time spent in the office designing a network survey.

- No additional survey data collected on benchmarks.

- No data processing using proprietary software.

Traditional OPUS-S required a minimum of 2 hours of static data to ensure most effective results. NGS has expanded OPUS capabilities to include sessions as short as 15 minutes by developing OPUS Rapid Static, or OPUS-RS, which will process data sets from 15 minutes to 2 hours (National Geodetic Survey, 2010b).

OPUS-RS solutions are computed differently than described above for OPUS-S. OPUS-RS takes six steps to resolve coordinates for a user's data file, and the primary processing is completed using Rapid Static GPS (RSGPS) software (Schwarz and others, 2009). Before running RSGPS, improved positional coordinates are determined for the location of the GNSS receiver by incorporating the nearest CORS. Once this is accomplished, RSGPS is run to determine integer ambiguities, tropospheric refraction parameters, and double difference ionospheric delays at the chosen CORS (Schwarz and others, 2009). Ionospheric delays are interpolated to the GNSS receiver position before a least squares adjustment is used to solve for the positional coordinates.

The first two steps in OPUS-RS are similar to OPUS-S; initial quality checks are performed on the GNSS data file and orbit files are retrieved. In the third step, OPUS-RS estimates the location of the GNSS receiver and determines the distance to each candidate CORS. A list that is sorted by distance is created, from which OPUS-RS attempts to retrieve the RINEX files for the time span of the GNSS data file. If a RINEX file is retrieved, it is evaluated for inclusion in the overall adjustment. This search continues down the list of candidate CORS until 9 reference stations have been located, the next reference station is greater than $250 \mathrm{~km}$ from the GNSS receiver, or 50 candidates have been examined.

OPUS-RS requires a minimum of three CORS stations within $250 \mathrm{~km}$ of the GNSS receiver to continue the analysis. In addition, the position of the GNSS receiver cannot be more than $50 \mathrm{~km}$ outside the polygon created by the selected CORS. The geometry of CORS and the distance to each CORS in relation to the GNSS receiver has a substantial impact on the quality of the final positional coordinates. OPUS-S and OPUSRS require data collection using a dual-frequency receiver.

\section{Quality Assurance}

Whether using OPUS-S or OPUS-RS, the user must consider solution quality requirements of the project. Typically, OPUS-S can resolve centimeter-level positions as indicated primarily by the peak-to-peak differences. The percent of observations used and ambiguities fixed, coupled with overall RMS, should be evaluated to assess the solution quality. Quality solutions from OPUS-S should have (National Geodetic Survey, 2011a):

- Less than $5 \mathrm{~cm}$ peak-to-peak errors.

- Greater than 90 percent observations used.

- Greater than 50 percent fixed ambiguities.

- RMS less than $3 \mathrm{~cm}$.

None of these quality checks are associated with systematic errors, such as the incorrect receiver antenna type or the wrong receiver antenna height being entered. The solution report created in OPUS-RS looks similar to the OPUS-S solution report; however, the OPUS-RS report (fig. 20) has a few different quality checks that the user needs to evaluate. Instead of a peak to peak error associated with each coordinate dimension, an uncertainty is calculated from the single baseline estimates from 3 to 9 CORS individually, and the final coordinate is computed from a least squares adjustment utilizing all CORS simultaneously. This uncertainty represents the square root of the differences between the single baseline estimates using CORS individually, and the final coordinate using least squares and all CORS. OPUS-RS produces a warning if the 


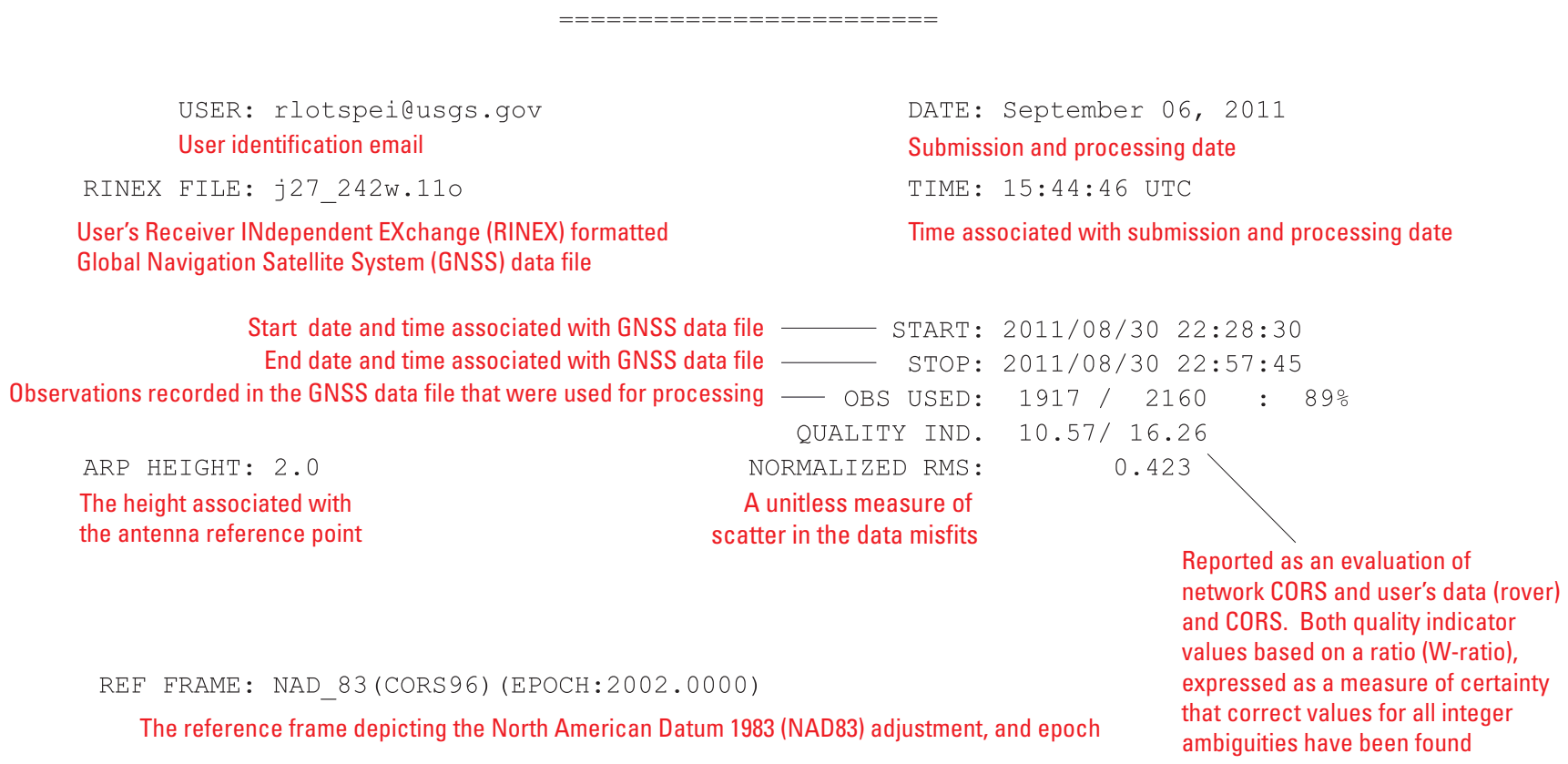

USER: rlotspei@usgs.gov

User identification email

RINEX FILE: j27_242w.110

User's Receiver INdependent EXchange (RINEX) formatted Global Navigation Satellite System (GNSS) data file

DATE: September 06, 2011 Submission and processing date TIME: 15:44:46 UTC Time associated with submission and processing date

REF FRAME: NAD_83 (CORS96) (EPOCH:2002.0000)

The reference frame depicting the North American Datum 1983 (NAD83) adjustment, and epoch

Reported as an evaluation of network CORS and user's data (rover) and CORS. Both quality indicator values based on a ratio (W-ratio), expressed as a measure of certainty that correct values for all integer ambiguities have been found

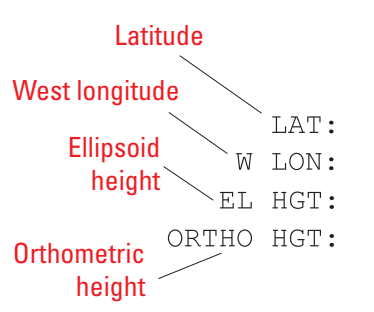

Estimated uncertainty [m, meters] $0.010(\mathrm{~m})$ $0.006(\mathrm{~m})$ $0.037(\mathrm{~m})$

$0.043(\mathrm{~m})$

North Americn Vertical Datum 1988

[NAVD88 (Computed using GEOID09)]

Orthometric conversion using the geoid hybrid model GEOID 09

UTM COORDINATES

STATE PLANE COORDINATES

$\begin{array}{llll}\text { Northing (Y) } & \text { [meters }] & 4094292.670 & 1074470.748 \\ \text { Easting (X) } & {[\text { meters }]} & 372831.242 & 3684360.487\end{array}$

Horizontal positional coordinates expressed as Universal Transverse Mercator (UTM) and State Plane

Continually Operating Reference Stations (CORS)

BASE STATIONS USED

\begin{tabular}{llllrr} 
PID & DESIGNATION & LATITUDE & \multicolumn{1}{c}{ LONGITUDE DISTANCE (m) } \\
DF3914 LOY1 LOYOLA 1 COOP CORS ARP & N370343.812 W0762412.356 & 8734.2 \\
DL1888 LOYZ LOYOLA Z CORS ARP & N365148.919 W0763424.809 & 18737.1 \\
DL3186 LOYX LOYOLA X CORS ARP & N371635.031 W0764143.820 & 39980.5 \\
DL1900 LSO3 LOYOLA LSO3 CORS ARP & N364719.436 W0755734.330 & 47233.7 \\
DL6896 NCEL ELIZABETH CITY CORS ARP & N362028.791 W0761529.274 & 73169.6 \\
DL3889 LOYW LOYOLA LOYW CORS ARP & N373140.995 W0755052.663 & 79201.6 \\
DL2314 LSO2 LOYOLA LSO2 CORS ARP & N371547.900 W0772439.913 & 92537.8 \\
DH7133 NCJA JACKSON NC CORS ARP & N362430.563 W0772615.309 & 110604.8 \\
DI2269 VAWI WALLOPS ISLAND CORS ARP & N375603.499 W0752815.949 & 135105.0
\end{tabular}

Nearest published benchmark as found in the National Geodetic Survey Integrated Database (NGS IDB) NEAREST NGS PUBLISHED CONTROL POINT
FX0258
J 27
N365911.
W0762544.
24.6

Figure 20. Modified output of a position generated from the Online Position User Service-Rapid Static (OPUS-RS). 
spread of the single baseline estimates are greater than $5 \mathrm{~cm}$ when comparing horizontal coordinates, or greater than $10 \mathrm{~cm}$ when comparing vertical coordinates. OPUS-RS also provides the normalized RMS for the final adjustment, which is a unitless measure of the scatter in the data misfits (National Geodetic Survey, 2010b). The final quality-control value that is provided on an OPUS-RS solution is a quality indicator that is based on a ratio (W-ratio) expressed as a measure of certainty that correct values for all integer ambiguities have been found (Schwarz, 2009). The quality indicator (W-ratio) depicted in the solution represents adjustments related to the network and rover. A general rule of thumb dictates that an indicator that is above 3 represents favorable geodetic quality solutions in which correct ambiguities are located, and those below 1 should be used with caution (Martin, 2007). In summary, a quality OPUS-RS solution should have a low uncertainty for each coordinate, a normalized RMS of 1 or less, and a quality indicator greater than 1 . Predicted solution qualities from 15-minute and 1-hour sessions across the country are provided at the following URL (http://geodesy.noaa.gov/OPUSI/Plots/ Gmap/OPUSRS_sigmap.shtml) and might be a useful tool when planning a survey.

To improve positional quality, a surveyor should collect longer observations (Eckl and others, 2001), observe at several different satellite geometries and average OPUS solutions from all observations, and wait to submit the data until rapid or precise orbits are available. Precise orbits are the evaluation of a complete Sunday through Saturday orbit and are available 10 to 14 days after the end of the GPS week. Before release of the precise orbit, satellite positions are described using the rapid orbit, which is available every 17 hours; the ultra-rapid orbit, available every 6 hours; or the broadcast orbits, which are the predicted satellite locations. Precise and rapid orbits are similar in quality such that users are typically not able to detect any differences (M. Schenewerk, National Geodetic Survey, written commun., 2011). For greater positional quality, CORS data can be downloaded by the user and post processed manually using software that performs baseline processing and network adjustment. OPUS solutions can also be checked and verified by including benchmarks. The verification of an OPUS solution can be done by collecting static data on a confident benchmark while other GNSS receivers are collecting static data. Static data collected on the benchmark can be processed through OPUS and compared to the known coordinates to verify that OPUS-derived orthometric heights were accurate when compared to benchmark elevations during the survey.

\section{Additions and Advancements}

The NGS has made additions to the OPUS processing, which include extended and Extensible Markup Language (XML) reports, and analysis of global positioning data outside the United States (with the exception of calculated orthometric heights) (National Geodetic Survey, 2011a). An extended OPUS report is five pages instead of the single page standard OPUS report and contains several pieces of information, including the final position in state plane coordinates in U.S. survey foot and meters, the position of each CORS used in the processing, and the position as determined from each CORS (Schenewerk, 2011). This additional information can be useful when looking at reducing peak-to-peak errors by incorporating or eliminating particular CORS. The XML report bounds each value by "tags" that uniquely identify it $(<\mathrm{TAG}>$ value $</ \mathrm{TAG}>)$ ) and can easily be read electronically. Another upgrade NGS has made to OPUS allows ellipsoid heights to be analyzed outside the conterminous United States (CONUS); however, OPUS currently does not have the functionality to determine orthometric heights outside of CONUS because these geoid models have not been well developed or maintained by NGS.

Beyond standard positional processing, NGS has developed additional tools for OPUS including the database or OPUS-DB. Originally, OPUS solutions were processed and sent by email to the user, but were not stored by NGS for future inquiry or reference. With the creation of OPUS-DB, the user who is processing 4 or more hours of data through OPUS-S now has the choice to store the solution to the NGS database. The database used by OPUS-DB is different than the NGS Integrated Database (IDB) used to query high accuracy reference marks because limited quality standards are set for publishing in OPUS-DB, and strict quality and processing standards must be met to publish a benchmark in NGS IDB. If the user chooses to store the results in OPUS-DB, standard identification information must be registered. When publishing a solution in OPUS-DB, the user can either describe a new mark or select a recovered mark using the NGS PID number. When describing a new benchmark, details such as name, stamping, type, depth, setting, location description, and photos are required. Additional information on benchmark stability, magnetic properties, application, and equipment used are optional. When entering a new benchmark into the OPUS-DB, the surveyor should consider the longevity of the location and the stability. A benchmark susceptible to movement or tampering for a shorter period of time would likely not be useful information to store in the database; however, storing benchmark information in the database is useful not only to the surveyor and others looking for benchmarks in the area, but also to the NGS who can use the information collected at the mark to enhance future geoid models.

A second tool in OPUS that was beta released in the year 2011 is OPUS-Projects (National Geodetic Survey, 2011a), which allows users to process OPUS solutions as a network that can be adjusted using CORS, PAGES, and ADJUST. In short, OPUS-Projects automates "bluebooking" [bluebooking is the processing and evaluation of GNSS survey data for publication in the IDB, and produces files needed to publish high accuracy benchmarks in the NGS IDB (Schenewerk, 2011)]. OPUS-Projects can provide better accuracy than OPUS-S and ensure that local networks are adjusted to minimize local errors (Armstrong, 2010). With an accessible and intuitive online user interface, OPUS-Projects provides benefit to those 
who do not process static networks on a regular basis; however, training by NGS is required for project managers. OPUSProjects allows the user to decide what autonomous observations and coordinates are used as control, and what baselines to process in the network adjustment (network adjustments are described in detail in the next section). OPUS-Projects will have user-specified quality checks and will highlight OPUS solutions that do not meet these checks.

Other future updates to OPUS include expanding capabilities from GPS-only data collection and processing to include all GNSS data (OPUS-GNSS). The inclusion of GNSS data processing will require replacement of the CORS GPS receiver antennas with GNSS receiver antennas as well as updating processing software, ensuring that ephemeris data are available in a timely fashion, and updating antenna model information. OPUS-GNSS is a primary focus for NGS (Rick Foote, NGS, oral commun., 2011). Two final future updates within OPUS are referred to as OPUS-Net (Schenewerk, 2011) and OPUS Leveling Online Computing Service (LOCUS). OPUS-Net will provide a network approach using a weighted least squares adjustment of nearby and distant CORS, and 10 nearest reference stations by the International GNSS Service (IGS) (Weston and Ray, 2011).

The use of OPUS has expanded greatly in the past several years. As a result, the NGS has worked to improve the usefulness of OPUS while ensuring quality in resolved positions. The use of OPUS permits a time savings to users because data collection only occurs at objective points. In addition, users have the ability to store and retrieve their processed observations, as well as search and retrieve benchmarks surveyed by others. With OPUS-Projects, users are able to complete network adjustments based on CORS, benchmarks, and OPUS-processed positions within the OPUS interface. Overall, OPUS is a tool that many USGS GNSS surveyors should become familiar with because of its applicability and quality positioning.

\section{Network Surveying and Processing}

Historically, global positioning used networks of fixed receivers continuously collecting data to augment static data collection at an objective point. With the development of RT corrections, this method is not as popular as it once was; however, regarding approach, post-processed network surveys continue to provide the least amount of uncertainty in a campaign. Networks may be either simple or complex and are often used where:

- Trusted benchmarks are separated by large distances from the project area.

- Geometric relation between CORS and the project area is lacking.

- Combined CORS and benchmarks are used to establish and check new benchmarks.
Networks might be used for many applications, including perpetuating datum to temporary locations, or to establish or interconnect groundwater wells, elevations at gaging stations, control for land subsidence and erosion studies, or project areas that require benchmark establishment for RT surveys.

Manual processing of static GNSS data can be done using benchmarks or CORS. Manual processing of static GNSS data with CORS may be done to improve accuracy for OPUS solutions or to reduce local errors between objective points. Manual processing with benchmarks may be done because few or distant active monuments are available, or just to improve local positioning. The manual processing of GNSS data occurs by way of a network, in which existing benchmarks are occupied by GNSS receivers collecting static data along the network exterior, whereas static data collection (objective points) occurs simultaneously within the network interior. Essentially, all observed benchmarks and objective points are integrated into a network of baselines that are processed together and adjusted to fit with the benchmark or control positions. This type of static surveying is discussed in terms of static and "fast static" surveying. The term "fast static" was historically used in practice to describe how higher grade dual-frequency antennas could be used to collect data for shorter periods of time (minutes compared to hours) to produce quality static survey results. The term is used less often in practice and is incorporated into the term "static" surveying because the methods are the same for shorter durations of time.

Network surveying provides the greatest accuracy by using multiple benchmark control. OPUS-S uses active monuments (CORS), but they are often tens of miles away, which reduces the accuracy of the solution because of long base lines (Skeen, 2005). In addition, OPUS-S processes each baseline individually, whereas network surveys use all benchmarks and objective points tied together by several baselines, which are analyzed together to correct the entire network. Network surveying is often used to establish new benchmarks within study areas where RT surveying is preferred but no high-order benchmarks exist, or objective points are spread through a large area where radio communication and baseline errors would make RT methods difficult.

\section{Network Control}

To ensure high-quality elevations, benchmarks of desirable quality should be included and processed within the network. Elevation at CORS stations and other GNSS-derived positions are based on ellipsoid heights. Models of the Earth's gravity (by way of a hybrid geoid model) are used to relate these ellipsoid heights to orthometric heights, and only a few active monuments have published orthometric heights at the time of this writing. Elevation that is generally considered as "truth" is known at thousands of benchmarks across the country from differential leveling surveys. Benchmarks should always be included when high-quality orthometric heights are desired. 
Developing a network survey that includes benchmarks can be challenging because many benchmarks have not been visited in years. Benchmarks are referred to as passive monuments because they are not active (that is continuously collecting data) like active stations such as CORS. All benchmarks were established with current coordinates at some time in the past, but because of crustal movement and geomorphic change, the published coordinates may no longer be accurate. Regardless, these benchmarks are still the foundation of elevation surveys and should be checked for accuracy when being used. NGS does provide epoch dates on any benchmark that is inside a horizontal crustal movement region, and therefore likely to move through time.

Another important difference to note between CORS and benchmarks is the horizontal datum. A datum can be defined mathematically by eight unique parameters (used to define the center, orientation, and reference ellipsoid) and realized in practice by the coordinates of a select set of points on the Earth. These parameters are referred to as a geodetic reference system, and its realization referred to as a geodetic reference frame (Lapine, 2010). The system is unique and exact whereas the frame is only as accurate as the data used to realize coordinates of the selected points on the Earth. As discussed earlier, NAD 83 is the current North American horizontal datum. The definition of NAD 83 is a set of eight parameters, but the reference frame was originally realized by a network of approximately 250,000 benchmarks and a sparsely-spaced network of TRANSIT (GPS system) satellite positions across North America in 1986 (Lapine, 2010). This first realization was named NAD 83 (1986) in recognition of the epoch date for the national adjustment. NAD 83 went through a series of readjustments using GPS surveying technology along with
CORS, making the realization more accurate (table 8). The datum and its realization are important when developing a network survey because all benchmarks used as primary control in a static survey should be in the same datum and realization.

As discussed earlier during mission planning, it is most effective to set time aside for benchmark recovery before final survey design and the actual campaign date because many benchmarks listed in the NGS database may not be recoverable or may not be conducive to GNSS data collection. Most network adjustment programs and reference material specify the minimum number of control points to include in a network as 3 horizontal controls and 3 to 4 vertical controls. Horizontal and vertical controls can be different benchmarks, or one benchmark with high-quality vertical and horizontal control. Quality horizontal coordinates could be established on vertical benchmarks by first processing the data collected through OPUS before inclusion in a network survey (Zilkoski and others, 1997). If additional benchmarks are included in the network, it is beneficial to allow them to be adjusted (as opposed to holding them fixed or using them as control in the adjustment) so that a comparison can be made between the known elevation of these additional benchmarks and adjusted elevations from the processed network. Essentially these additional benchmarks can serve as a verification of the final quality.

Benchmark accuracy may be confusing because different types of benchmarks are described differently; some use proportional accuracy and others use relative accuracy. Proportional accuracy relates accuracy as a function of distance, and relative accuracy describes either a radius or linear value at a 95-percent confidence interval from a least squares adjustment representing how the survey point fits within the control

Table 8. NAD 83 realizations, marks used in each realization, and comments about each adjustment.

[Modified from W. Henning, 2010 and D. Doyle, National Geodetic Survey, written commun., 2011. NAD 83, North American Datum of 1983; HARN, High Accuracy Reference Network; GPS, Global Positioning System; CORS, Continually Operating Reference Station; FBN, Federal Based Network; CBN, Cooperative Based Network; NSRS, National Spatial Reference System; NGS, National Geodetic Survey; GNSS, Global Navigation Satellite System; IGS, International GNSS Service]

\begin{tabular}{|c|c|c|}
\hline NAD 83 realization & Marks used in the realization & Comments \\
\hline NAD 83 (1986) & Triangulation and trilateration data & Original adjusment; horizontal only. \\
\hline NAD 83 (HARN) & State-by-state GPS-derived data & Pre-CORS, but added ellipsoid heights. \\
\hline NAD 83 (CORS96) epoch 2002 & CORS & $\begin{array}{l}\text { Nationwide adjustment, did not include benchmarks, } \\
\text { reflects active monumentation velocities rather than a } \\
\text { snapshot in time. }\end{array}$ \\
\hline NAD 83 (NSRS2007) epoch 2007 & $\begin{array}{l}\text { GPS campaign data on 70,000 benchmarks } \\
\text { between 1995-2005 and CORS }\end{array}$ & $\begin{array}{l}\text { No classically derived data included. CORS constrained } \\
\text { to their estimated values for January } 1,2007 \text {, } \\
\text { accounting for vertical motion of CORS through time. } \\
\text { No vertical motion model for the benchmarks was } \\
\text { included. }\end{array}$ \\
\hline
\end{tabular}


network. With the 2007 readjustment of NAD 83 (NSRS 2007), NGS has started reporting relative accuracies on benchmarks that previously only represented proportional accuracy. Most accuracy standards are given in orders, but orders differ depending on the survey performed, whether leveling (table 9) or GPS (table 10).

Table 9. Accuracy standard for leveled, triangulated, and traversed benchmarks (National Geodetic Survey, 2001a).

[Horizontal standard, refers to the maximum closure error; vertical standard, relative accuracy between directly connected benchmarks; NA, not applicable; $\mathrm{mm}$, millimeters; $\sqrt{ }$, square root; $\mathrm{K}$, the distance in kilometers between benchmarks]

\begin{tabular}{lcc}
\hline \multicolumn{1}{c}{ Order } & Horizontal standard & Vertical standard \\
\hline First & 1 part in 100,000 & NA \\
First Class I & NA & $0.5 \mathrm{~mm} \sqrt{\mathrm{K}}$ \\
First Class II & NA & $0.7 \mathrm{~mm} \sqrt{\mathrm{K}}$ \\
Second Class I & $1: 50,000$ & $1.0 \mathrm{~mm} \sqrt{\mathrm{K}}$ \\
Second Class II & $1: 20,000$ & $1.3 \mathrm{~mm} \sqrt{\mathrm{K}}$ \\
Third Class I & $1: 10,000$ & $2.0 \mathrm{~mm} \sqrt{\mathrm{K}}$ \\
Third Class II & $1: 5,000$ & $\mathrm{NA}$ \\
\hline
\end{tabular}

Table 10. Accuracy standard for Global Positioning System (GPS)derived benchmarks (National Geodetic Survey, 2001a).

[Horizontal standard, refers to the minimum geometric accuracy standard based on the distance between benchmarks (or baselines) at the 95-percent confidence level; ellipsoid standard, the maximum height difference accuracy calculated from an minimally constrained, correctly weighted, least squares adjustment; cm, centimeter; NA, not applicable]

\begin{tabular}{lcc}
\hline \multicolumn{1}{c}{ Order } & Horizontal standard & Ellipsoid standard \\
\hline AA & $0.3 \mathrm{~cm}+1: 100,000,000$ & NA \\
A & $0.5 \mathrm{~cm}+1: 10,000,000$ & NA \\
B & $0.8 \mathrm{~cm}+1: 1,000,000$ & NA \\
First & $1 \mathrm{~cm}+1: 100,000$ & NA \\
First Class I & NA & 0.5 \\
First Class II & NA & 0.7 \\
Second Class I & $2.0 \mathrm{~cm}+1: 50,000$ & 1 \\
Second Class II & $3.0 \mathrm{~cm}+1: 20,000$ & 1.3 \\
Third & $5.0 \mathrm{~cm}+1: 10,000$ & NA \\
Third Class I & NA & 2 \\
Third Class II & NA & 3 \\
Fourth Class I & NA & 6 \\
\hline
\end{tabular}

The accuracy of the relation of a benchmark described as first order to that of the control from which it was surveyed is 1 part for every 100,000 (table 9). In other words, if the benchmark is 13,000 meters from the control point, the accuracy of that benchmark in relation to the control is 0.13 meters or 13,000/100,000. Likewise, the vertical order describes the vertical relation between benchmarks as determined by the distance between benchmarks in kilometers. Therefore, the accuracy of the vertical relation between benchmarks that are $4.8 \mathrm{~km}$ apart and described as first-order class II, is $1.5 \mathrm{~mm}$ or:

$$
0.7 \mathrm{~mm} \sqrt{4.8}
$$

Accuracy standards regarding GNSS-derived benchmarks are determined by baseline length and the quality of the minimally constrained least squares adjustment (table 10).

\section{Planning}

Once recovered, useable, and trusted benchmarks that surround objective points are located, planning of the data collection effort and network can begin. First, as discussed in mission planning, a map of all objective points and benchmarks (control) should be drafted; then, baselines to be observed can be sketched out (fig. 21) and repeat baselines can be coordinated with appropriate time offsets, generally 24 hours plus several hours so that different atmospheric conditions and a different satellite geometry are observed (Zilkoski and others, 1997, 2008).

When planning a large survey with many benchmarks and objective points, the survey can be broken up into smaller areas, as long as those areas allow stations and baselines to interconnect the overall network. Figure 21 illustrates a large network, which is surveyed in three smaller areas (sessions A-C). Baselines processed within each area are drawn. Depending on the number of receivers and the quality of the survey or occupation time on each objective point and benchmark, this survey could be accomplished in one day. A re-observation could be accomplished the next day as a quality check during different atmospheric conditions and satellite geometry. Marks in the small area (session A) would be re-observed in reverse order so the marks that were surveyed in the evening during the first observation are now surveyed in the morning during the second observation. When planning a network survey, the quality of the final adjustment is strongly affected by the distance from the benchmarks held fixed to those interior objective points, the number and quality of the benchmarks, the length of time each objective point is observed, the number and time offset of re-observations, and the geometry of the benchmarks in relation to the objective points; therefore, all these aspects should be considered and evaluated when designing a network. Essentials of a quality static network survey design for obtaining high-quality ellipsoid and orthometric heights are described by Zilkoski and others $(1997,2008)$. During this planning period, travel time should be factored into the campaign schedule so that there is sufficient time between sessions for each surveyor to move to a new location, locate the benchmark, and properly setup the GNSS receiver and receiver antenna.

Previously, it was recommended that similar GNSS receiver antennas (manufacturer and model) be used during a static surveying campaign to ensure consistency to the antenna phase center and prevent any variations during processing. The antenna phase center is the position 

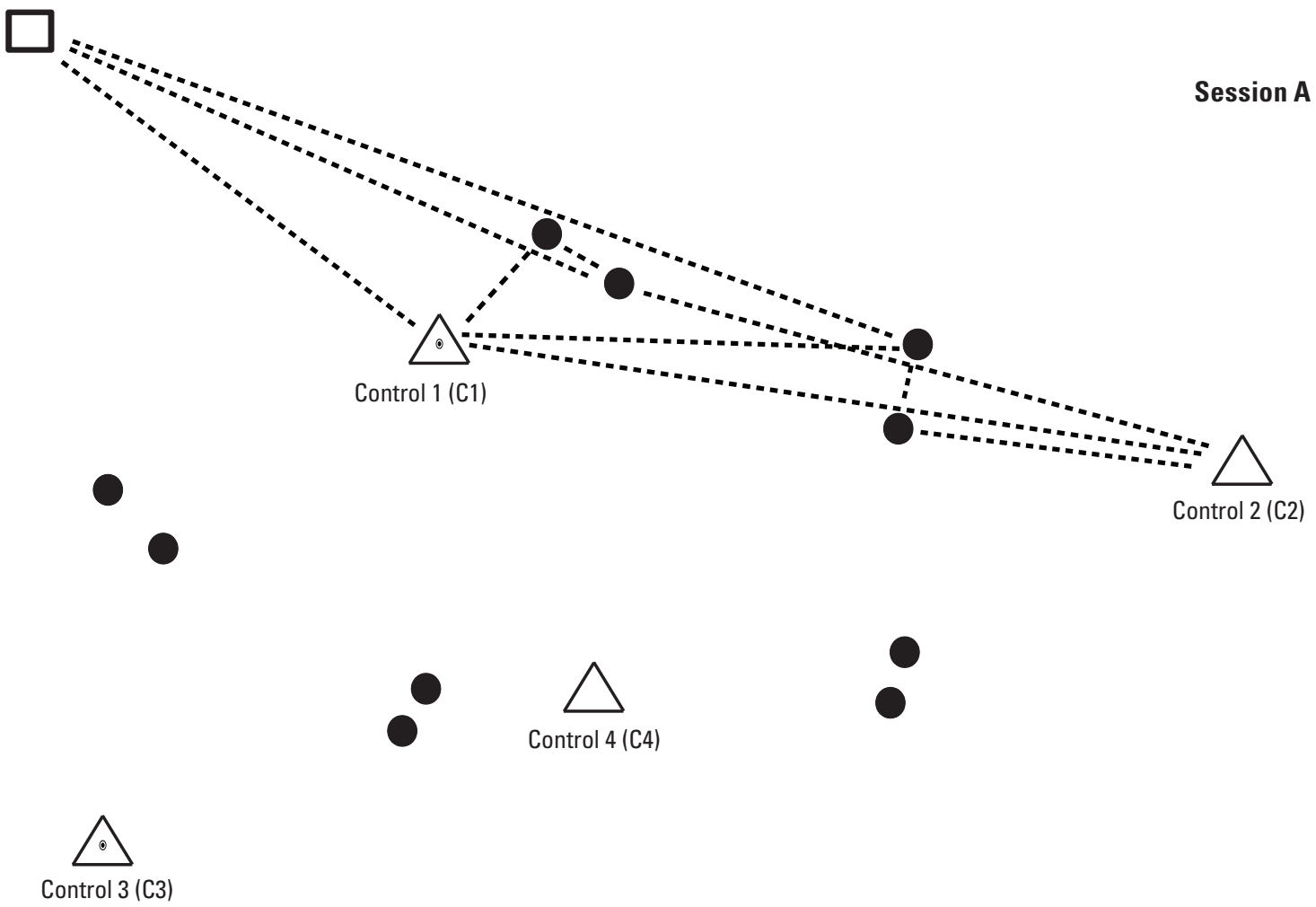

100 KILOMETER

\begin{tabular}{|c|c|}
\hline \multicolumn{2}{|c|}{ Observation schedule } \\
\hline Session & Control stations \\
\hline A & C1, C2 \\
B & C1, C2, C4 \\
C & C1, C4, C3 \\
\hline
\end{tabular}

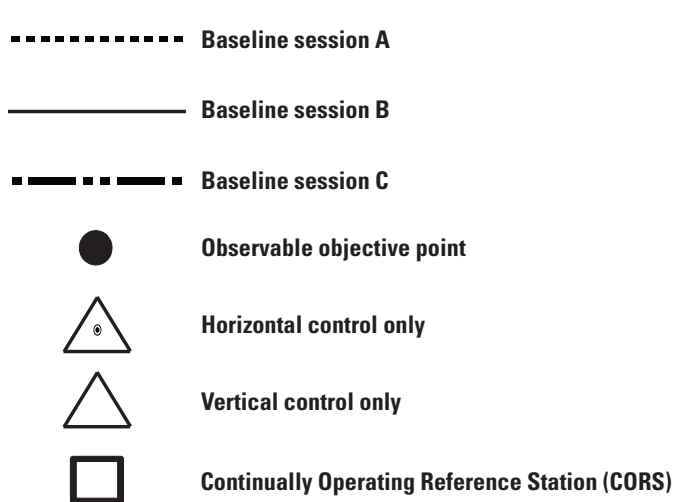

Figure 21. Simple network design with resurveys and baselines. 
$\square$
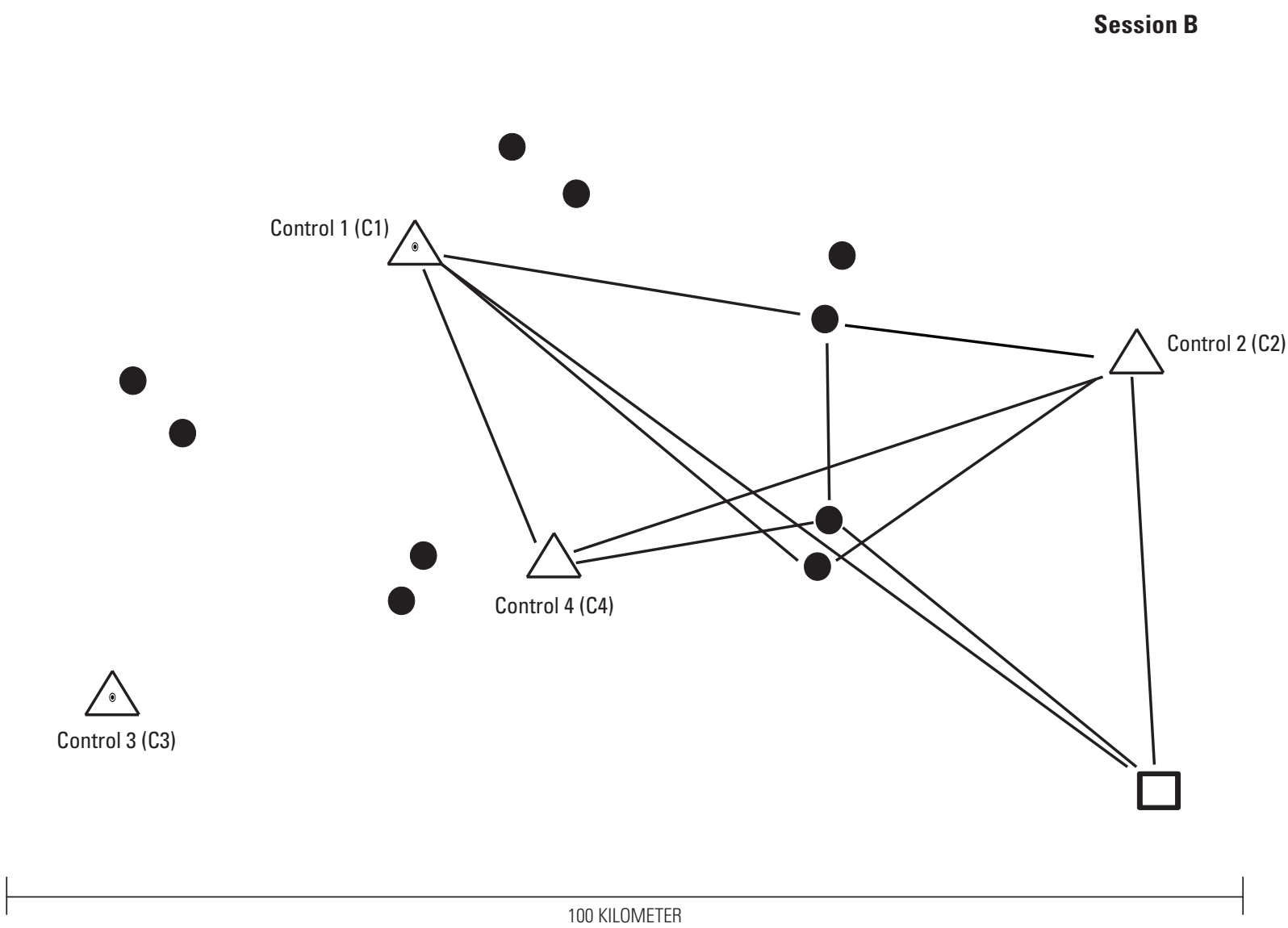

EXPLANATION

\begin{tabular}{|c|c|}
\hline \multicolumn{2}{|c|}{ Observation schedule } \\
\hline Session & Control stations \\
\hline A & C1, C2 \\
B & C1, C2, C4 \\
C & C1, C4, C3 \\
\hline
\end{tabular}

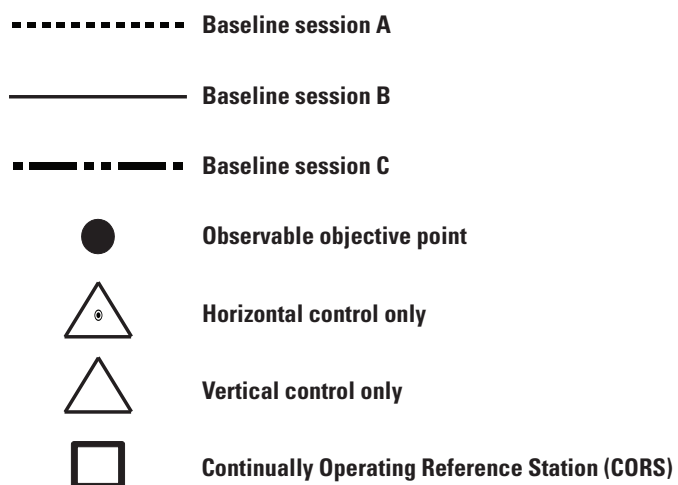

Figure 21. Simple network design with resurveys and baselines.-Continued 


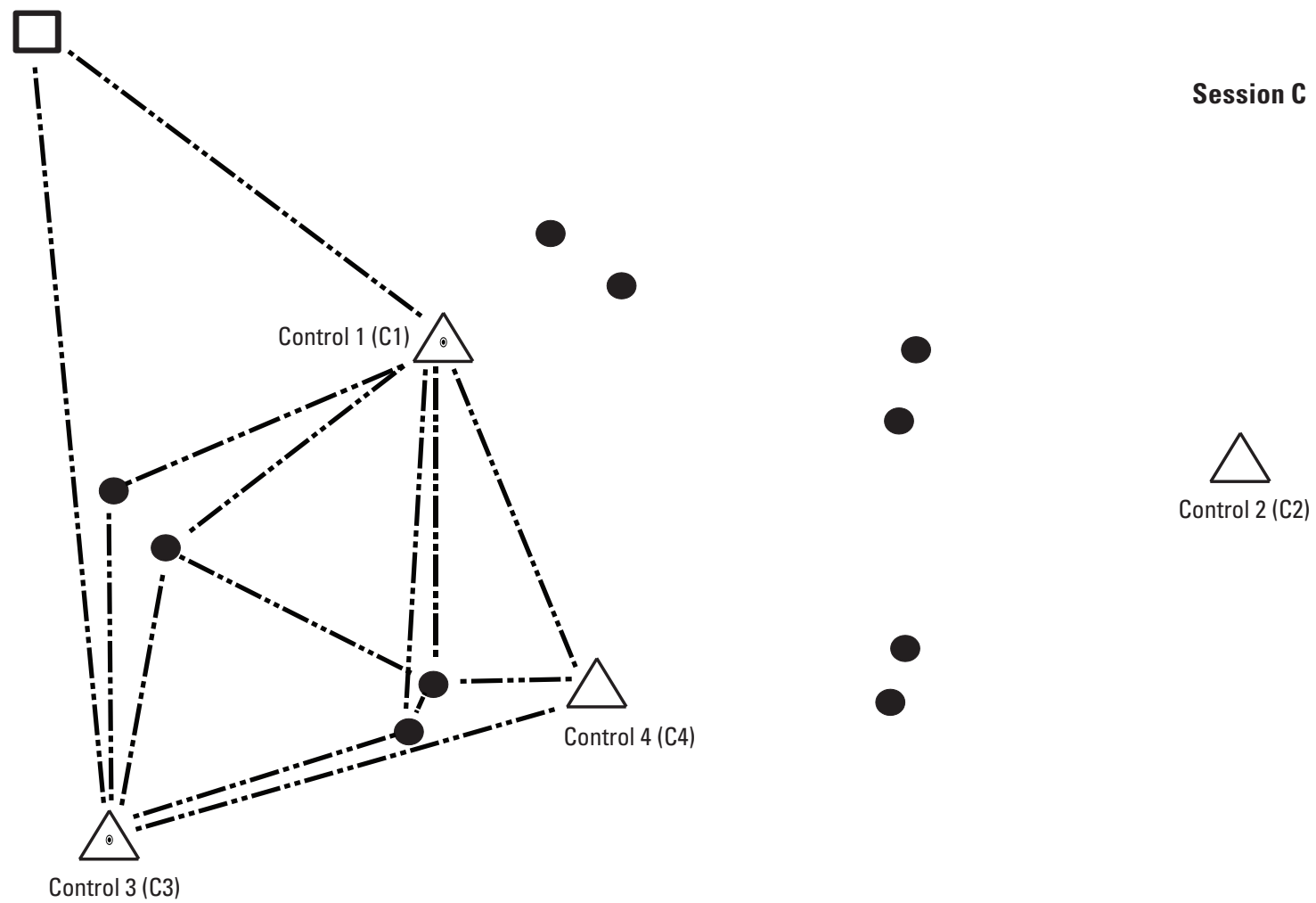

Control 3 (C3)

EXPLANATION

\begin{tabular}{|c|c|}
\hline \multicolumn{2}{|c|}{ Observation schedule } \\
\hline Session & Control stations \\
\hline A & C1, C2 \\
B & C1, C2, C4 \\
C & C1, C4, C3 \\
\hline
\end{tabular}

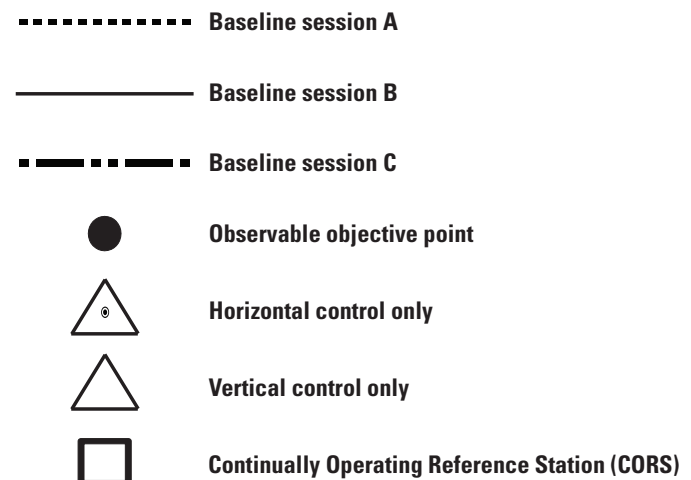

Figure 21. Simple network design with resurveys and baselines.-Continued 
of the precise point being measured when measurements between satellites and the receiver antenna are being made (Mader, 1999). The phase center is neither a physical point nor a stable point. Most of the variation in the phase center depends on the satellite elevation, changing with direction of the satellite signal. This is important to understand, because antenna offsets are needed to correct GNSS measurements to physical monuments. It has been indicated that phase center offsets and variations are important to model and do affect the quality of the final coordinates, especially vertical coordinates (Mader, 1999). Antenna phase center offsets are important when manually processing GNSS data because some GNSS receiver antennas do not have well-defined antenna phase offsets, and not all software has pre-set phase center offset information for all antennas. Therefore, if several receiver antenna types are used in a survey, the phase center offsets must be entered manually into the processing software or selected from a list of predefined receiver antennas. When discussing, applying, and learning about phase center offsets, note that phase centers are currently measured and described as absolute values or vectors from the antenna reference point to the $\mathrm{L}_{1}$ and $\mathrm{L}_{2}$ average phase center (Mader, 2010). Previously, phase centers were measured in relative terms as a comparison to a standard antenna. This measurement method is no longer the method that is used, so a surveyor must be sure that the offsets being applied are absolute (in relation to the antenna reference point) and not relative (in relation to a standard antenna). Phase center variations describe how the average phase center varies depending on satellite angle above the horizon. Many static post-processing programs have phase center information associated with each selectable receiver antenna type to ensure the phase center offset is accounted for. In addition, if the program being used does not have information pre-programmed on the specific receiver antenna, the surveyor can usually find the phase center offset information on the NGS website (Mader, 2010) and work with technical support for NGS or the post-processing program to add the antenna and phase center offset information.

Once a network survey has been designed, including several repeat baselines and multiple observations on objective points at different satellite geometries, the survey chief must organize surveying personnel and provide them with information on the planned campaign. The survey chief should ensure the surveying team has maps of the campaign area, the survey itinerary, and a list of contacts for other surveyors, those in the field and in the office. The team should also go through mission planning considerations, as mentioned earlier in the "Mission Planning and Errors" section, to verify which survey times have sufficient satellite coverage with low PDOP, review CORS data that they plan to use to verify that the station is currently functioning properly, and contact landowners or other local contacts that need to be informed of the work.

\section{Processing and Adjustment}

Manual processing for static surveys, compared to OPUS processing or no post processing, permits the user to control many factors that affect the quality of the results. These factors include ephemeris used, time span of collected data, number and location of satellites used (modified by elimination of poor satellites from an observation or adjustment of an elevation mask), and selection and quality of baselines that are processed. A detailed review of each observation session can determine if additional observations are necessary. Processing network survey data typically follows these general steps:

1. A loop closure analysis.

2. Unconstrained network adjustment of all base lines.

3. Analysis of all baselines and repeat baselines in the unconstrained adjustment.

4. Correction or elimination of baselines with poor results or poor fit with other baselines.

5. Fully constrained adjustment on remaining baselines.

Unconstrained and fully constrained network adjustments are least squares adjustments. Simply put, a least squares adjustment incorporates the exact baseline parameters or the position of each objective point and benchmark (that holds the control positions fixed) in the network, and computationally results in the least amount of error. The usefulness of the method rests in part upon the mathematical demonstration that if the errors in the measurements of any quantity follow a few reasonable laws, the most probable value of the quantity is the one for which the sum of the squares of the residual errors (or corrections) is a minimum. If the observations are of unequal weight, then the most probable value is the one for which the sum of the squares of the weighted residuals is a minimum (National Geodetic Survey, 2001b). The weighting used in a least squares adjustment varies between different adjustment software and can be controlled by the user in some software packages. The most common weighting method in a least squares adjustment is to use the reciprocal of the variance of the quantity to multiply and increase or decrease the effect of that quantity on the results of an adjustment (National Geodetic Survey, 2001b). In other words, baselines with lower solution variance will have more effect in the adjustment than baselines with greater solution variance. Full understanding of the least squares adjustment is not necessary to adjust a GNSS network; however, the user does need to have knowledge of the software and quality checks and tests that are performed so an evaluation of the adjusted coordinates can be made.

The first step in a network adjustment is to bring the survey data into the processing software. When entering data into a processing software package, care must be taken to verify each data set has the correct receiver antenna $\left(\mathrm{L}_{1}\right.$ and $\mathrm{L}_{2}$ offsets), correct receiver antenna height, and the correct data 
collection time span. If CORS data are entered as part of the network, the metadata for each site should be reviewed and checked to make sure the receiver antenna type entered is correct (note that superseded receiver antenna types are published in the metadata and are common at many CORS stations). In addition, an ephemeris file will need to be loaded for each survey day in the network and all benchmarks need to have known coordinates entered regardless of whether or not the benchmark will be held as a control (fixed point). Most software manuals will have a full section devoted to network adjustment that should be read before performing an adjustment. After entering all survey data into the adjustment project, the baselines are selected and processed. The processed baselines are then run through a loop closure test. The loop closure test is a process for determining the amount of error in a set of observations within a network (Trimble Business Center, 2011). The results of the loop closure test are then evaluated to determine whether errors in the loop closure could be reduced by identifying and removing inconsistent baselines (those that are not fitting well in the network) or duplicate baselines that conflicted with one another. Any poor baseline can either be eliminated or the data at each observation can be evaluated to reduce the noise or error. Observation times may be eliminated from the beginning or intermittently throughout if segments of the file represent poor satellite data during the observation.

Once all loops have closed within acceptable standards, a minimally constrained adjustment can be performed. A network adjustment that uses the minimum number of constraints required to define the coordinate system is representative of a minimally constrained adjustment. This step is performed to measure the internal consistency among observations (Trimble Business Center, 2011). Before performing the minimally constrained adjustment, one control benchmark must be coded in the software as a fixed position so the entered coordinates for that benchmark will not change within the dataset during the adjustment. The minimally constrained adjustment may identify any data outliers that do not fit within the overall network. In addition, the adjustment can be evaluated by observing how far benchmarks with known coordinates (but not held fixed in the adjustment) were shifted from the known position.

The final step after the minimally constrained adjustment has been completed and all accuracy tests and checks have been accepted, is to perform a fully constrained adjustment. A fully constrained adjustment involves the adjustment of all points in the network that are part of a larger control network held fixed to their published coordinate values. Essentially a fully constrained adjustment may be used to combine smaller and larger networks as well as older and newer networks (Trimble Business Center, 2011). To perform the fully constrained adjustment, several benchmarks should be coded as fixed or control positions in addition to the benchmark already held fixed in the minimally constrained adjustment. If additional benchmarks have been included in the network that are not coded as fixed or control positions, they may be used after the adjustment to evaluate the final network solution by reviewing how far the positions of each benchmark have been shifted from published positions. In addition to coding the benchmarks as control positions, a geoid model must also be selected. Once all these steps have been performed, a fully constrained adjustment can be run and the final results (including all statistical tests and checks) should be reviewed once again. Statistical tests run by each software are typically well described in the software manual and should be well understood so that the results of the adjustment have meaning. A common way to evaluate the final quality of the fully adjusted network is to use benchmarks that were not used as control as described above, evaluate the 95-percent confidence limits given for each position as provided by the software, and evaluate PPM (parts per million) error in the baselines. PPM error is a calculation of the error based on the length of the baseline; longer baselines inevitably have more error than short baselines. The positions for the fully constrained adjustment are the final positions determined for each objective point. Network processing steps are further demonstrated in appendix 4 .

\section{Post-Processed Kinematic Surveying}

Post-processed kinematic surveying is another method of post processing global positioning data. Post-processed kinematic surveying was originally used when a radio link from the base station to the rover was not available. This surveying method requires the base station to be set up stationary, at a known or unknown location, while the rover unit is used to collect autonomous GNSS observations on objective points and benchmarks. The base data and the rover data are then post processed in the office to increase the accuracy of the rover positions based on the data collected at the base station. This method is not typically used because RT approaches are progressively more available, and accuracy checks and evaluations are able to be done in real time; however, this method is still facilitated in many post processing software packages and handheld data collection devices. This method may be useful when radio or cellular communication is a problem, but the objective points are within a reasonable distance of the base station so PPM errors are not induced. Unfortunately, real-time accuracy checks are limited to the evaluation of satellite data at the rover only; therefore, there would be no indication of the quality of data being collected at the base station or the quality of the final solution until the data was post processed.

\section{GNSS Quality}

The concept of accuracy for any GNSS campaign can be complex. There are several components tied to GNSS surveys that reflect some degree of quality. Aside from most effective surveying practices including occupation time, redundancy, and the use of high-accuracy active and passive stations (benchmarks), there are underlying components, such as the quality of the ellipsoid, the hybrid geoid model, and the instrumentation used. 
The establishment of vertical control using GNSS techniques should utilize trusted benchmarks. The definition of a trusted benchmark should be a mark that has the assurance of quality and stability, and represents a current datum and adjustment. A localization to benchmarks provides a reference against "the truth." These trusted benchmarks on the ground have been used to develop the hybrid geoid model, which in turn is used to convert ellipsoid heights, derived from GNSS positioning, to orthometric heights. It is not recommended that the definition of "the truth" represent orthometric heights derived solely by active stations using the current hybrid geoid model; however, there are geographic areas without sufficient availability of heights derived by leveling, thus requiring GNSS derived heights. Generally, the NGS encourages all active stations be aligned with the NSRS that represents a current and consistent datum and adjustment, for example NAD 83 (2011), epoch 2010.0. Alignment and consistency of active stations permits a quality standard that can be used from one locality to another without discrepancy. The NGS recognizes quality horizontal positioning that is derived by active stations alone, but expresses concerns regarding vertical positioning as the quality of the hybrid geoid model may vary from one project area to the next (D. Doyle, National Geodetic Survey, oral commun., 2011). Benchmarks are used to provide assurance as a quality check, such that the accuracy of a survey may be derived as the residual between the benchmark and the GNSS solution. Without the consideration of a benchmark, and all other assurances being equal, one has to assume an accurate and consistent hybrid geoid model to provide accurate heights. Geoid models, as discussed in the "Geodesy Background" section, are developed from a combination of gravity data, ellipsoid heights, and leveled heights. These models are more problematic in areas susceptible to disturbance, subsidence, tectonic movement, uplift, and seasonal variations, and are therefore updated periodically to reflect these changes. The use of active stations to derive an orthometric height, without a localization to trusted benchmarks, puts the reliability on the geoid model, a model that may have limitations based on the above-mentioned areas. At the time of this writing, the NGS is continuing to utilize all disseminated ellipsoid heights on existing benchmarks to improve the accuracy of the geoid (National Geodetic Survey, 2001c). One method the NGS is utilizing to facilitate these ellipsoid heights derived on existing benchmarks is by using data submitted through OPUS-DB.

For network surveys, trusted benchmarks of quality vertical order are needed to provide a framework in which to constrain and yield objective points. Considering the purpose and scope of this manual, horizontal positioning is of marginal concern. Active stations are utilized during a network survey and a horizontal position may be derived using observations at vertical benchmarks or objective points within the network. This may be an adequate means of obtaining a horizontal component necessary (but not primary) to the purpose and scope of this manual. Once again, the benchmark is held in high regard to ensure a quality network survey.
It is difficult to ascertain a numerical representation of accuracy for GNSS approaches in establishing vertical datum. There are, however, different assurances and approaches that may be categorized to represent a level of survey-grade GNSS quality toward data collection. These have been categorized as Level I, Level II, Level III, and Level IV, and are summarized in table 11. Note that each level of quality assumes most effective field practices, such as:

- Bubble check and calibration of base fixed-height tripod or rover bipod.

- Base fixed-height or dual-clamped tripod stabilized by chains or sandbags.

- Mission planning, including base or rover multipath avoidance for each observation session.

- Height of base Antenna Reference Point (ARP) measured before and after observation session.

Additionally, it is the responsibility of the GNSS user to ensure benchmarks in the project area are sufficiently checked during a campaign, and those checks need to be spatially representative of all data collection as much as possible. In this regard, the benchmark continues to remain the optimal representation of truth.

GNSS-derived benchmarks are common in many parts of the country. As a result, it is important to note a distinction between these derived benchmarks (height modernization marks) and benchmarks derived by leveling (such as first-, second-, and third-order). The quality categories in table 11 identify benchmarks derived by leveling (for example, second- and third-order) as recommendations for the origination of geodetic work; however, there may be circumstances where benchmarks derived from leveling do not exist or, if they do exist, cannot be trusted, and those marks explicitly derived by GNSS are available. It is incumbent upon the surveyor to use the highest quality benchmarks available. There is an evaluation method difference between GNSS-derived benchmarks and leveled benchmarks; the uncertainty of GNSS-derived benchmarks is evaluated in an absolute sense, whereas the leveled benchmarks are evaluated in a relative sense. Regardless, the surveyor should be aware of the published elevation and its decimal expression. At a minimum, benchmarks used for geodetic work to establish datum should be expressed to the centimeter level.

\section{Level I Survey}

The Level I survey is regarded as the highest quality survey that may be engaged by USGS staff [a higher standard published by the NGS "Guidelines for Establishing GPSDerived Orthometric Heights-NOAA Technical Memorandum NOS NGS 59" is available by the NGS for those who almost always wish to achieve local accuracies of $2 \mathrm{~cm}$ to $5 \mathrm{~cm}$ (Zilkoski and others, 2008)]. The Level I survey also can be categorized as a network survey or a single-base static survey (table 11). 
Table 11. Level-quality descriptions for Global Navigation Satellite System (GNSS) positioning in the U.S. Geological Survey.

$[\leq$, less than or equal to; km, kilometer; +, approach excessive; \%, percent; OPUS-S, Online Position Users Service-Static; RMS, Root Mean Square; m, meter; OPUS-RS, Online Position Users Service-Rapid Static; -, approach insufficient; RTK, Real-Time Kinematic; RT, real time; PDOP, Position Dilution of Precision; $\geq$, greater than or equal to]

\begin{tabular}{|c|c|c|c|c|}
\hline $\begin{array}{l}\text { Network surveys } \\
\end{array}$ & Level I & Level II & Level III & Level IV \\
\hline Baseline length; vertical order 2 benchmarks & $\leq 40 \mathrm{~km}$ of each other & $\leq 60 \mathrm{~km}$ of each other & + & + \\
\hline Baseline length; objective points & $\leq 15 \mathrm{~km}$ of benchmarks and each other & $\leq 25 \mathrm{~km}$ of benchmarks and each other & + & + \\
\hline Occupation time; vertical order 2 benchmarks & 4 hour minimum & 4 hour minimum & + & + \\
\hline Occupation time; objective points & 1 hour minimum & 1 hour minimum & + & + \\
\hline Number of controls: vertical order 2 benchmarks & 4 & 4 & + & + \\
\hline Number of checks on benchmarks & Any available & Any available & + & + \\
\hline Baseline redundancy: objective points & $50 \%$ & $50 \%$ & + & + \\
\hline \multicolumn{5}{|l|}{ Single-base OPUS-S surveys* } \\
\hline Occupations & 2 & 1 & 1 & + \\
\hline Duration & 4 hour minimum & 4 hour minimum & 2 to 4 hour & + \\
\hline Observations used & $\geq 80 \%$ & $\geq 80 \%$ & $\geq 60 \%$ & + \\
\hline Ambiguities fixed & $\geq 80 \%$ & $\geq 80 \%$ & $\geq 60 \%$ & + \\
\hline Solution RMS error & $\leq 0.03 \mathrm{~m}$ & $\leq 0.03 \mathrm{~m}$ & $\leq 0.05 \mathrm{~m}$ & + \\
\hline Vertical peak-to-peak & $\leq 0.06 \mathrm{~m}$ & $\leq 0.08 \mathrm{~m}$ & $\leq 0.10 \mathrm{~m}$ & + \\
\hline Assurance check & Average within $0.05 \mathrm{~m}$ of each solution & + & + & + \\
\hline
\end{tabular}

Assurance check

Average within $0.05 \mathrm{~m}$ of each solution

\section{Single-base OPUS-RS surveys*}

\section{Occupations}

Duration

Solution RMS error

Orthometric height estimated uncertainty

\section{Real-time positioning**}

Base station occupations for single-base RTK

RT blunder check

PDOP

Collection interval

Satellites

Baseline distance

Vertical precision (2 sigma)

Objective point assurance checks

Benchmark checks or localization

1
1 to 2 hour
Normalized between 0.1 and 1
$\leq 0.08 \mathrm{~m}$

1

Yes, within $0.03 \mathrm{~m}$

$\leq 3$

1 -second interval for 3 minutes (180 epochs)

$\geq 7$

$\leq 9 \mathrm{~km}$

$0.05 \mathrm{~m}$

15 min to 2 hour

Normalized between 0.1 and 1

$\leq 0.10 \mathrm{~m}$

Average within $0.05 \mathrm{~m}$ of each solution Average within $0.05 \mathrm{~m}$ of each solution +

\section{for $10 \%$ of observations}

for $10 \%$ of observations

Yes, vertical order 2 within $0.05 \mathrm{~m}$

*Derived from National Geodetic Survey (NGS)-OPUS, 2010.

${ }^{* *}$ Derived from Henning, 2010. 
The Level I network survey is a derivation from Zilkoski and others (2008), which requires a quantity of four benchmarks of second-order vertical accuracy or better within a 40-km spacing of each other. Objective points within the network must reside within a $15-\mathrm{km}$ spacing of the previously described benchmarks and each other. Occupation time must be a minimum of 4 hours for all held benchmarks and no less than 1 hour for all interior objective points. For the Level I network, 50 percent of the objective points need to be double occupied, and it is recommended that any additional benchmarks (if available) exceeding the required four that are held fixed be included in the network as a quality check at a minimum occupation time of 1 hour. Observations on benchmarks initially held fixed do not require re-observation. The length of the campaign will likely ensure enough variation in atmospheric conditions and satellite geometry for objective point re-observations suitable for USGS science and data collection; however, although not required, it is suggested that all benchmarks (held fixed) and 50 percent of the objective points be re-observed at a time offset, generally greater than 24 hours. Processing results from both of these sessions may be averaged.

The Level I single-base static survey essentially adopts guidelines provided by NGS OPUS. Although there are other software utilities that provide more autonomy for post processing solutions, such as manual editing and deletion of outliers, OPUS is a well maintained and widely accepted utility from the NGS that provides sufficient functionality for use within the USGS. For single-base static surveys using OPUS, Level I criteria require two occupations: the observation of consecutive days, performed at different times of the day, to ensure variability of atmospheric conditions (for example morning session day one, afternoon session day two). Processing for these single-base surveys is facilitated by OPUS-S, which utilizes NGS PAGES software to process dual-frequency observation files. Observation times should be no less than 4 hours, at least 80 percent of the total observations in the data file should be used, at least 80 percent of ambiguities should be fixed (resolved) in the solution, the vertical peak-to-peak error should not exceed $0.06 \mathrm{~m}$, and the root mean squared (RMS) error of the solution should not exceed $0.03 \mathrm{~m}$. Additionally, the average of both OPUS solutions must be within $0.05 \mathrm{~m}$ of either solution used to average.

GNSS ground-control points are often established to evaluate the accuracy of airborne Light Detection And Ranging (LiDAR) data used. The USGS draft LiDAR version 13 specifications (U.S. Geological Survey, 2010) refer to the National Digital Elevation Program (NDEP) guidelines for digital elevation data, part 1 , as the source for determining elevation accuracy. The NDEP guidelines recommend a minimum of 20 ground-control points for major vegetation types that are dependent upon the area being collected such as open terrain, tall weeds and crops, brush lands and low trees, forested areas fully covered by trees, and urban areas (National Digital Elevation Program, 2004). LiDAR guidelines and base specifications established by the U.S. Geological Survey National Geospatial Program reference NDEP guidelines for digital elevation data. For NDEP purposes, it is identified that the independent source or ground-control point should maintain an accuracy at least 3 times greater than the digital data set being evaluated at the 95-percent confidence level (National Digital Elevation Program, 2004). As an example, for products depicting 1-foot contour intervals, the vertical accuracy of the data set should be no greater than $18.2 \mathrm{~cm}$ according to the National Standard for Spatial Data Accuracy (NSSDA) (National Digital Elevation Program, 2004). Given a target accuracy of $6.1 \mathrm{~cm}(18.2 \mathrm{~cm} / 3$ $=6.1 \mathrm{~cm})$, it is incumbent upon the GNSS user to select an approach that satisfies this requirement. A Level I quality category would likely be optimal in this case and would require baseline redundancy as part of the network approach, a subsequent observation as part of a single-base OPUS-S approach, and trusted monumentation for either approach within this category to further validate and ensure this $6.1-\mathrm{cm}$ uncertainty among established ground control.

\section{Level II Survey}

A Level II survey may consist of a static network survey, a single-base static survey, or a real-time (RT) survey including a single-base or network approach (table 11). The network survey for Level II requires a quantity of four benchmarks of second-order vertical accuracy or better within a $60 \mathrm{~km}$ spacing of each other. Objective points within the network must reside within a $25-\mathrm{km}$ spacing of the previously described benchmarks and each other. Occupation time must be a minimum of 4 hours for all held benchmarks and no less than 1 hour for all interior objective points. For the Level II network, 50 percent of the objective points need to be double occupied and it is recommended that any additional benchmarks (if available) exceeding the required four that are held fixed, be included in the network as a check at a minimum occupation time of 1 hour.

Single-base static surveys, as part of a Level II survey, are derived from National Geodetic Survey OPUS and require observations times to be no less than 4 hours, at least 80 percent of the total observations in the data file should be used, at least 80 percent of ambiguities in the solution should be fixed, the vertical peak-to-peak error should not exceed $0.08 \mathrm{~m}$, and the root mean squared (RMS) error of the solution should not exceed $0.03 \mathrm{~m}$.

A single-base rapid static approach may also be considered Level II quality. As a result of the increasing amount of CORS, OPUS-RS provides acceptable solution qualities sufficient to satisfy the constraints of a Level II survey. All OPUSRS solutions must ensure duration times no less than 1 hour, a normalized RMS value between 0.1 and 1 , and an estimated uncertainty of the orthometric height no greater than $0.08 \mathrm{~m}$.

The quality requirements of a Level II survey also include a RT approach using benchmarks and RT blunder checks described earlier in the "Single-base RTK" section. The Level II RT approach is derived from Henning (2010), which requires rover redundancy, in which observation lengths shall be no less than 180 epochs (1-second data collection interval for 3 minutes). The RT baseline lengths should not exceed $9 \mathrm{~km}$ (with the exception of RTNs that have an interpolation 
methodology involving a virtual base station, which essentially provide a "virtual" base station near each observation), PDOP should not exceed 3, and observable satellites should be greater than or equal to 7 . The difference between redundant observations during a RT blunder check should not exceed $0.03 \mathrm{~m}$, and vertical precision from the base station (or RTN virtual reference station) to the rover (usually identified at the bottom of the screen on the data collector) for each observation should be within $0.05 \mathrm{~m}$ at 2 sigma. A localization of 2 trusted benchmarks of second-order vertical accuracy or better should be performed where 1 benchmark is used to move the hybrid geoid model up or down to align the vertical datum (also known as 2-point site calibration), and the other is used as a quality check (Henning, 2010). To reduce the effects of multipath through averaging, a final observation should be performed on the beginning benchmark.

For trusted benchmarks available within a project area not to exceed $10 \mathrm{~km}$, an evaluation of benchmarks may be performed by entering those benchmarks into the data collector, conducting RT observations over those benchmarks, then performing a localization (site calibration) for all of the benchmarks to evaluate how well they fit. It is the responsibility of the GNSS user to recognize limitations of localizations and exceeding baseline lengths to benchmarks that are used as a quality check away from objective points. As previously discussed within RTN quality assurance, a calibration scale factor remains fixed and unchanging, whereas the projection scale factor maintains a rate of change as the distance increases. For this reason, a $10-\mathrm{km}$ baseline should exist, and for project areas that extend beyond this distance, a subdivision might be necessary to "split" localizations. When splitting project areas for separate locations, it is recommended that a common tie exist from one area to the next.

Only 2 benchmarks are required for a localization (1 to localize and 1 to check), so other marks used in the initial localization may be ignored, particularly marks determined to be outliers. From this information, the user can select which benchmark to use for localization and which benchmark to use when performing a check (fig. 22).

If the user does not wish to perform a localization, quality checks on benchmarks should be performed throughout the survey to spatially represent the project area as much as possible. Whether a user is performing a localization or just checking benchmarks, a quality check on a benchmark needs to be performed at the beginning and end of a Level II campaign. Campaigns involving the establishment of 10 or more objective points must ensure reoccupation of 10 percent of those objective points before performing a quality check at the end of the campaign. Quality checks on benchmarks and reoccupied objective points should not vary more than $0.05 \mathrm{~m}$.

As an example, a single-base RTK approach is undertaken within a project area that does not exceed $10-\mathrm{km}$ baselines. The user occupies the base receiver at an autonomous location and enters four trusted benchmarks (second-order vertical accuracy or better) recovered in the project area, into the data collector. The user then operates the rover to conduct redundant observations (as part of a RT blunder check) at a 1-second interval for 3 minutes over each benchmark as part of a localization. The user then evaluates benchmark residuals and selects 1 mark to localize, and at least 1 mark to check (while turning off or deleting outliers). It is important to note that a minimum of

Vertical residuals produced by a least squares fit of known benchmarks to Global Navigation Satellite System (GNSS) observations at those known benchmarks

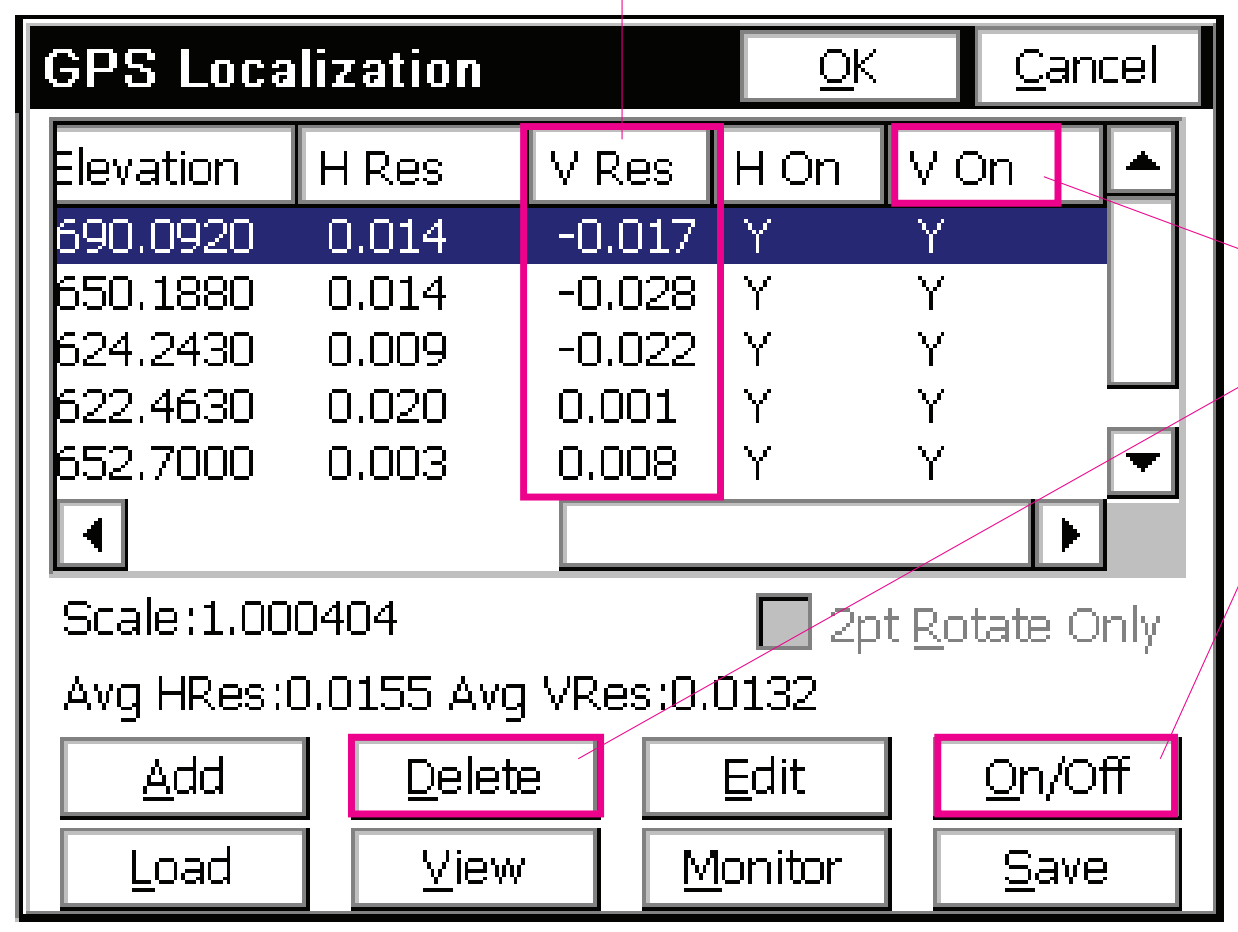

Figure 22. Example results as displayed in the data collector of a localization with Global Navigation Satellite System (GNSS). 
four benchmarks should be entered into the data collector to ensure computation and display of vertical residuals within the site calibration utility. If there are less than four benchmarks available in a particular project area, residuals can be assessed manually outside of the site calibration utility. While administering RT blunder checks, the user ensures that values representing vertical precision from the base station to the rover (usually identified at the bottom of the screen on the data collector) for each observation are within $0.05 \mathrm{~m}$ at 2 sigma. These redundant observations are performed over one benchmark selected as a quality check or localization in the beginning of the campaign, such that the elevation of the first observation is within $0.03 \mathrm{~m}$ of the second observation and the average is within $0.05 \mathrm{~m}$ of the stated benchmark elevation. [Note that when using an autonomous base location, the observed elevation will be equivalent to the known elevation for the beginning benchmark check. The ending quality check will ensure continuity among both benchmarks so that any error may be distributed equally among beginning and ending benchmarks. The distribution of error among beginning and ending benchmarks, including all bounded objective points, is difficult to assign using an autonomous RT approach. An attempt to proportion error to each observation and benchmark based on observation quality parameters would be subjective (outside of the ensured quality assurance needed for a Level II or Level III RT approach); therefore, it is most effective to distribute error equally among all benchmarks and bounded objective point observations]. The user then observes all objective points in the project area while adhering to RT blunder checks within $0.03 \mathrm{~m}$ as described above. At the end of the campaign, the user reoccupies 10 percent of the objective points (for campaigns that establish 10 or more objective points) and conducts a quality check on another benchmark in the same manner as the quality check at the beginning of the campaign. Finally, the user returns to the benchmark initially observed for the final observation and conducts a quality "closing" check in the same manner as the first two benchmark observations. This final "closing" check should reside within $0.05 \mathrm{~m}$ of the stated benchmark elevation.

As another example, a RTN approach is undertaken within a $30-\mathrm{km}$ wide project area. The user evaluates the 30-km area by locating usable benchmarks of second-order vertical accuracy or better, and appropriately subdivides the $30-\mathrm{km}$ area to ensure localization to 1 benchmark and a quality check to another benchmark in each subdivision. As previously discussed, as a general rule, the subdivisions should not exceed $10 \mathrm{~km}$ in length. The user recovers and enters six trusted benchmarks (second order or better recovered in the project area) into the data collector. The user then operates the rover to conduct redundant observations (as part of a RT blunder check) at a 1-second interval for 3 minutes over a trusted benchmark as part of a localization in the first subdivision. If more than 2 trusted benchmarks are recovered, the user then evaluates benchmark residuals and selects 1 mark to localize, and at least 1 mark to check (while turning off or deleting outliers) in the first subdivision. While administering RT blunder checks, the user ensures values representing vertical precision from the base station to the rover (usually identified at the bottom of the screen on the data collector) for each observation is within 0.05 $\mathrm{m}$ at 2 sigma. These redundant observations are performed over one benchmark selected as a quality check or localization in the beginning of the campaign, such that the elevation of the first observation is within $0.03 \mathrm{~m}$ of the second observation and the average of both are within $0.05 \mathrm{~m}$ of the benchmark elevation. The user then observes all objective points in the subdivision while adhering to RT blunder checks within $0.03 \mathrm{~m}$ as described above. At the end of the campaign, the user reoccupies 10 percent of the objective points (for campaigns that establish 10 or more objective points) and conducts a check on another benchmark in the same manner as the check in the beginning of the campaign. Finally, the user returns to the benchmark initially observed for the final observation and conducts a quality "closing" check in the same manner as the first two benchmark observations. Reoccupation of objective points and benchmark checks must be within $0.05 \mathrm{~m}$ from the initial objective point and benchmark elevation. The user repeats the same procedures for the second and third subdivisions of the $30-\mathrm{km}$ project area. Summarized observations provided in table 12 for this example validate the assurance of Level II criteria.

\section{Level III Survey}

The Level III survey includes single-base static and rapid-static surveys, and a modified RT approach (table 11). The quality of a network survey exceeds the requirements of a Level III survey. For a Level III survey, the single-base static approach is depreciated from a Level II survey, such that the OPUS-S solution still requires observation times between 2 to 4 hours, but only 60 percent of the total observations in the data file must be used, 60 percent of ambiguities in the solution should be fixed, the vertical peak-to-peak error should not exceed $0.1 \mathrm{~m}$, and the root mean squared (RMS) error of the solution should not exceed $0.05 \mathrm{~m}$.

A single-base rapid static approach may satisfy the constraints of a Level III quality. All OPUS-RS solutions may have observation times between 15 minutes to 2 hours, normalized RMS values between 0.1 and 1 , and yield an estimated uncertainty of the orthometric height no greater than $0.1 \mathrm{~m}$.

The RT approach for a Level III survey maintains the same requirements as the Level II survey with some exceptions, as outlined in table 11 .

\section{Level IV Survey}

Post-processed GNSS solutions, such as network surveys or single-base static surveys, represent qualities that exceed a Level IV survey. A Level IV survey represents real-time approaches that do not meet criteria for the Levels I-III surveys mentioned above; however, a Level IV survey is generally reserved for RT surveys that require fixed solutions without localizations, redundancies, checks, or guidelines regarding baseline length, satellite availability, data collection 
Table 12. An example of summarized observations and quality criteria upheld for a Level II real-time (RT) survey using a Real-Time Network (RTN).

[m, meter; PDOP, Position Dilution of Precision; UTM, Universal Transverse Mercator coordinate system; Zone 15, UTM Grid designated every six degrees of longitude; BMXXXX, benchmark identifier; NA, not applicable; \%, percent]

\begin{tabular}{|c|c|c|c|c|c|c|c|c|c|c|c|c|}
\hline Mark & $\begin{array}{l}\text { Rover } \\
\text { receiver } \\
\text { height } \\
\text { (m) }\end{array}$ & $\begin{array}{l}\text { Vertical } \\
\text { precision } 2 \\
\text { sigma } \\
\text { (m) }\end{array}$ & $\begin{array}{c}\text { Average } \\
\text { number } \\
\text { of } \\
\text { satellites }\end{array}$ & PDOP & $\begin{array}{l}\text { Northing; } \\
\text { UTM, } \\
\text { Zone 15, } \\
\text { m }\end{array}$ & $\begin{array}{c}\text { Easting; } \\
\text { UTM, } \\
\text { Zone 15, } \\
\text { m }\end{array}$ & $\begin{array}{c}\text { GNSS } \\
\text { observation } \\
\text { elevation } \\
\text { (m) }\end{array}$ & $\begin{array}{c}\text { RT } \\
\text { blunder } \\
\text { check } \\
\text { (m) }\end{array}$ & $\begin{array}{c}\text { Final } \\
\text { elevation } \\
\text { (m) }\end{array}$ & $\begin{array}{l}\text { Vertical } \\
\text { order II } \\
\text { or better } \\
\text { benchmark } \\
\text { elevation } \\
\text { (m) }\end{array}$ & $\begin{array}{l}\text { Difference between final } \\
\text { elevation and bench- } \\
\text { mark elevation and } \\
10 \text { percent reoccupied } \\
\text { objective point } \\
\text { elevations } \\
\text { (m) }\end{array}$ & $\begin{array}{l}\text { Localization or } \\
\text { benchmark or } \\
\text { double } \\
\text { occupy check } \\
\text { (m) }\end{array}$ \\
\hline Benchmark-BM1187 & 1.7 & 0.01 & 14 & 1.1 & 4086421.351 & 301335.594 & 97.889 & -0.011 & 97.895 & 97.925 & $97.895-97.925=$ & -0.030 \\
\hline Benchmark-BM1187-check & 1.4 & 0.02 & 15 & & 4086421.353 & 301335.597 & 97.900 & & & & & \\
\hline Objective point 1 & 1.4 & 0.016 & 13 & 1.2 & 4083702.346 & 298559.472 & 95.840 & -0.001 & 95.841 & NA & NA & NA \\
\hline Objective point 1 -check & 1.7 & 0.01 & 14 & & 4083702.344 & 298559.470 & 95.841 & & & & & \\
\hline Objective point 2 & 1.7 & 0.024 & 16 & 1.5 & 4080400.402 & 303415.513 & 90.256 & 0.003 & 90.255 & NA & NA & NA \\
\hline Objective point2-check & 1.4 & 0.023 & 16 & & 4080400.405 & 303415.511 & 90.253 & & & & & \\
\hline Objective point3 & 1.4 & 0.024 & 17 & 1.2 & 4080400.402 & 303415.513 & 97.596 & -0.012 & 97.602 & NA & NA & NA \\
\hline Objective point3-check & 1.7 & 0.021 & 15 & & 4080400.406 & 303415.514 & 97.608 & & & & & \\
\hline Objective point4 & 1.7 & 0.012 & 14 & 1.0 & 4080400.403 & 303415.507 & 91.425 & -0.021 & 91.436 & NA & NA & NA \\
\hline Objective point4-check & 1.4 & 0.016 & 13 & & 4080400.407 & 303415.501 & 91.446 & & & & & \\
\hline Objective point5 & 1.4 & 0.01 & 15 & 1.0 & 4083587.763 & 305280.611 & 91.446 & -0.017 & 91.455 & NA & NA & NA \\
\hline Objective point5-check & 1.7 & 0.016 & 15 & & 4083587.767 & 305280.614 & 91.463 & & & & & \\
\hline Objective point6 & 1.7 & 0.018 & 16 & 1.2 & 4083587.764 & 305280.617 & 98.370 & -0.010 & 98.375 & NA & NA & NA \\
\hline Objective point6-check & 1.4 & 0.023 & 12 & & 4083587.761 & 305280.612 & 98.380 & & & & & \\
\hline Objective point7 & 1.4 & 0.011 & 18 & 1.0 & 4087641.677 & 302926.158 & 99.243 & -0.019 & 99.253 & NA & NA & NA \\
\hline Objective point7-check & 1.7 & 0.021 & 17 & & 4087641.682 & 302926.159 & 99.262 & & & & & \\
\hline Objective point 8 & 1.7 & 0.01 & 16 & 1.6 & 4087641.691 & 302926.152 & 91.877 & -0.007 & 91.881 & NA & NA & NA \\
\hline Objective point8-check & 1.4 & 0.018 & 15 & & 4087641.693 & 302926.157 & 91.884 & & & & & \\
\hline Objective point 9 & 1.4 & 0.018 & 16 & 1.8 & 4087641.694 & 302926.163 & 93.350 & 0.006 & 93.347 & NA & NA & NA \\
\hline Objective point9-check & 1.7 & 0.012 & 14 & & 4087641.634 & 302926.166 & 93.344 & & & & & \\
\hline
\end{tabular}


Table 12. An example of summarized observations and quality criteria upheld for a Level II real-time (RT) survey using a Real-Time Network (RTN).-Continued

[m, meter; PDOP, Position Dilution of Precision; UTM, Universal Transverse Mercator coordinate system; Zone 15, UTM Grid designated every six degrees of longitude; BMXXXX, benchmark identifier; NA, not applicable; $\%$, percent]

\begin{tabular}{|c|c|c|c|c|c|c|c|c|c|c|c|c|}
\hline Mark & $\begin{array}{l}\text { Rover } \\
\text { receiver } \\
\text { height } \\
\text { (m) }\end{array}$ & $\begin{array}{l}\text { Vertical } \\
\text { precision } 2 \\
\text { sigma } \\
\text { (m) }\end{array}$ & $\begin{array}{c}\text { Average } \\
\text { number } \\
\text { of } \\
\text { satellites }\end{array}$ & PDOP & $\begin{array}{c}\text { Northing; } \\
\text { UTM, } \\
\text { Zone 15, } \\
\text { m }\end{array}$ & $\begin{array}{c}\text { Easting; } \\
\text { UTM, } \\
\text { Zone 15, } \\
\text { m }\end{array}$ & $\begin{array}{l}\text { GNSS } \\
\text { observation } \\
\text { elevation } \\
\text { (m) }\end{array}$ & $\begin{array}{c}\text { RT } \\
\text { blunder } \\
\text { check } \\
\text { (m) }\end{array}$ & $\begin{array}{c}\text { Final } \\
\text { elevation } \\
\text { (m) }\end{array}$ & $\begin{array}{l}\text { Vertical } \\
\text { order II } \\
\text { or better } \\
\text { benchmark } \\
\text { elevation } \\
\text { (m) }\end{array}$ & $\begin{array}{l}\text { Difference between final } \\
\text { elevation and bench- } \\
\text { mark elevation and } \\
10 \text { percent reoccupied } \\
\text { objective point } \\
\text { elevations } \\
\text { (m) }\end{array}$ & $\begin{array}{l}\text { Localization or } \\
\text { benchmark or } \\
\text { double } \\
\text { occupy check } \\
\text { (m) }\end{array}$ \\
\hline Objective point10 & 1.7 & 0.012 & 13 & 1.8 & 4087641.700 & 302926.158 & 94.489 & 0.007 & 94.486 & NA & $\mathrm{NA}$ & NA \\
\hline Objective point10-check & 1.4 & 0.016 & 13 & & 4087641.707 & 302926.160 & 94.482 & & & & & \\
\hline Objective point11 & 1.4 & 0.02 & 18 & 1.4 & 4085329.687 & 300166.748 & 92.333 & -0.007 & 92.337 & NA & NA & NA \\
\hline Objective point11-check & 1.7 & 0.015 & 17 & & 4085329.692 & 300166.751 & 92.340 & & & & & \\
\hline Objective point12 & 1.7 & 0.016 & 18 & 1.2 & 4085329.689 & 300166.757 & 94.509 & 0.010 & 94.504 & NA & NA & NA \\
\hline Objective point12-check & 1.4 & 0.015 & 14 & & 4085329.684 & 300166.761 & 94.499 & & & & & \\
\hline Objective point13 & 1.4 & 0.02 & 14 & 1.6 & 4072504.710 & 294629.477 & 98.769 & 0.030 & 98.754 & NA & NA & NA \\
\hline Objective point13-check & 1.7 & 0.021 & 16 & & 4072504.710 & 294629.469 & 98.739 & & & & & \\
\hline Objective point14 & 1.7 & 0.025 & 17 & 1.9 & 4069130.573 & 289731.719 & 94.755 & -0.006 & 94.758 & NA & NA & NA \\
\hline Objective point14-check & 1.4 & 0.028 & 15 & & 4069130.574 & 289731.719 & 94.761 & & & & & \\
\hline Objective point 15 & 1.4 & 0.019 & 16 & 2.0 & 4072390.700 & 299443.333 & 92.387 & -0.003 & 92.389 & NA & NA & NA \\
\hline Objective point15-check & 1.7 & 0.013 & 15 & & 4072390.688 & 299443.329 & 92.390 & & & & & \\
\hline $\begin{array}{l}\text { Objective point } 1-10 \% \\
\text { Reoccupy }\end{array}$ & 1.7 & 0.008 & 17 & 1.2 & 4083702.342 & 298559.474 & 95.857 & 0.022 & 95.846 & & $95.841-95.846=$ & -0.005 \\
\hline $\begin{array}{l}\text { Objective point } 1-10 \% \\
\text { Reoccupy check }\end{array}$ & 1.4 & 0.009 & 18 & & 4083702.340 & 298559.472 & 95.835 & & & & & \\
\hline $\begin{array}{l}\text { Objective point6-10\% } \\
\text { Reoccupy }\end{array}$ & 1.4 & 0.006 & 18 & 1.4 & 4083587.763 & 305280.621 & 98.345 & -0.016 & 98.353 & & $98.375-98.353=$ & 0.022 \\
\hline $\begin{array}{l}\text { Objective point6-10\% } \\
\text { Reoccupy check }\end{array}$ & 1.7 & 0.007 & 17 & & 4083587.760 & 305280.616 & 98.361 & & & & & \\
\hline Benchmark-BM1186 & 1.7 & 0.013 & 16 & 1.4 & 4065570.643 & 288987.605 & 97.570 & 0.002 & 97.569 & 97.537 & $97.569-97.537=$ & 0.032 \\
\hline Benchmark-BM1186-check & 1.4 & 0.015 & 15 & & 4065570.640 & 288987.606 & 97.568 & & & & & \\
\hline Benchmark-BM1187 & 1.4 & 0.018 & 16 & 1.1 & 4086421.352 & 301335.593 & 97.920 & 0.017 & 97.912 & 97.925 & $97.912-97.925=$ & -0.013 \\
\hline Benchmark-BM1187-check & 1.7 & 0.020 & 18 & & 4086421.354 & 301335.594 & 97.903 & & & & & \\
\hline
\end{tabular}


interval, and observation time. At a minimum, one should try to ensure a PDOP value less than or equal to 6 . A summary of all level-quality surveys is tabulated in table 11 .

\section{Level Quality Alternatives with Real-Time Positioning}

The NGS has reduced the effort toward establishing traditional passive benchmarks. With the movement toward active stations for geodetic control, benchmarks by other entities are not being established at the rate they once were. In addition, a substantial percentage of existing benchmarks are disturbed by anthropogenic effects or geophysical effects (deformation, subsidence, crustal motion). As a result, it may be difficult to find a benchmark, particularly one of acceptable vertical quality. The quality described in the preceding section is established such that at a minimum, a Level III survey can be performed for data collection or project needs. In the absence of benchmarks with second-order vertical accuracy or better for a Level II survey, the user can substitute Level I OPUSderived control, which can be localized and checked as part of a RT campaign; however, OPUS-derived control for a RT campaign will depreciate the quality of a Level II survey to a Level III survey. As discussed earlier, a localization to trusted benchmarks established on the ground provides the most optimal representation of the ground surface, as opposed to the current hybrid geoid model and its associated uncertainties. Additionally, if only third-order benchmarks exist, a Level III survey is the highest quality that can be attained.

High-water mark surveys using GNSS may employ or combine several different quality surveys. For instance, project control may be set using a RT approach for a Level II or Level III survey. This control may be considered the foundation established for use of a total station used to survey high-water marks or additional topography canopied by trees; however, open topography that is part of the high-water mark survey may be surveyed with a Level IV quality approach, without previously described assurances.

\section{Uncertainty Analysis}

Surveys involving trusted benchmarks allow a simple comparison between GNSS observations and monumented elevations as a method of evaluating uncertainty. GNSS surveys involving active stations are more complex because many different parameters are evaluated as a function of "truth." OPUS-S solutions provide a peak-to-peak assessment, which (as described earlier in the "Single Base: Online Positioning User Service (OPUS)" section) is the difference between the maximum and minimum value of a coordinate obtained from the three baseline solutions from active stations. This peak-to-peak value is often considered an acceptable method of assessing uncertainty. For OPUS-RS solutions, uncertainty may be assessed by quality indicators, standard deviation of the processed coordinate, and a normalized RMS. Generally, a normalized RMS value outside of the range 0.1 to 1 is a sufficient indicator of noise or uncertainty in the processed coordinate. OPUS-S and OPUS-RS observations that are processed over a trusted benchmark provide a sufficient comparison between the processed OPUS solution and the known benchmark elevation. A comparison of processed OPUS solutions to trusted benchmarks ensures continuity among historic data collection derived from benchmarks, and provides another sense of solution accuracy; therefore, observations over benchmarks are recommended during single-base static campaigns utilizing OPUS.

Network surveys are based on constrained benchmarks, which are evaluated in terms of residuals in the processing report of the overall solution. In these surveys, the benchmark is paramount and weight should be applied accordingly based on reliability. Additional benchmarks occupied within the network (beyond the requirements described in Level I and Level II surveys) are good practice to ensure quality checks.

The acceptable uncertainty for the survey data used as the basis for the science should be evaluated before a particular level quality of survey is selected. A GNSS survey is only one component of error introduced into the final product. The end user of the data must evaluate different components of uncertainty, and the potential for these components to be additive and compound throughout the process to the final product. A GNSS user needs to ensure that the approach being used does not violate the degree of uncertainty one is trying to achieve or maintain in the process. An example would be the survey of high-water marks used as the basis for peak flow computations. The foundation of an indirect determination of peak discharge is based on the quality of high-water marks and the general setting of natural or artificial control features that distinguish the effectiveness of the approach. Considering the distinction of coastal storm surge and upland river high-water marks provided (table 13; L. Bohmann, U.S. Geological Survey, written commun., 2011), the use of a RT approach to directly survey high-water marks should be undertaken with caution because good or excellent marks may represent uncertainties ( 0.02 to $0.1 \mathrm{ft}$; table 13) below those inherent in a RT approach. For this example, the user should recognize error that stems from the complexity of the general setting and computational procedure, but be aware of the additive or worst case amount of uncertainty that can be derived overall in the process.

Table 13. Uncertainty of high-water marks for coastal storm surge and upland rivers.

[HWM, high-water mark; ft, feet; >, greater than]

\begin{tabular}{lcc}
\hline $\begin{array}{c}\text { HWM } \\
\text { classification }\end{array}$ & $\begin{array}{c}\text { Coastal storm surge } \\
\text { HWM uncertainty } \\
\text { (ft) }\end{array}$ & $\begin{array}{c}\text { Upland rivers } \\
\text { HWM uncertainty } \\
\text { (ft) }\end{array}$ \\
\hline Excellent & 0.05 & 0.02 \\
Good & 0.1 & 0.05 \\
Fair & 0.2 & 0.1 \\
Poor & 0.4 & 0.2 \\
Very poor & $>0.40$ & $>0.20$ \\
\hline
\end{tabular}


A similar USGS Water-Mission Area example may be applied when relating altitude among a groundwater well field with a RT approach. The user needs to have an awareness of the uncertainty of the potentiometric surface as well as an anticipated expression of the accuracy of altitude in the Ground-Water Site-Inventory System (GWSI) database (U.S. Geological Survey, 2004). Well data from small alluvialbased well fields without relief, and data used to assess groundwater and surface water interactions might require more precise methods of establishing altitude from one well to another. For these circumstances, the user needs to make sure that the uncertainty of the approach does not compromise the altitude accuracy achieved to evaluate the potentiometric surface.

Criteria established for the Level I, II, and III classifications are set forth to ensure a scale of quality in the approach. On occasion, some of the criteria set forth in each of the classifications may not be achieved for the anticipated level of quality. It is incumbent upon the user to exercise judgment in these cases by evaluating all criteria throughout the entire campaign. For example, during a Level II RT survey, 0.03-m assurance as part of the blunder check for three objective points were not met. If there were only 3 objective points in the survey, the blunder check assurance might be an issue and a potential downgrade to a Level III; however, if there were 30 objective points in the survey, the issue may be trivial. Continuing with this example, other assurances should be evaluated such as a $0.05-\mathrm{m}$ benchmark check, satellite availability, PDOP, and vertical precision (2 sigma). The GNSS user should have a sufficient understanding of these criteria and the perspective of their application to form a judgment regarding the final classification of survey held.

As previously discussed, the expression of numerical accuracy representing a GNSS survey is a culmination of many components. For cooperative agencies or public interests that desire a numerical accuracy of the GNSS survey, NSSDA, published by the Federal Geographic Data Committee (1998), is an accepted mathematical standard. The NSSDA method computes the vertical root mean square error (RMSE) of survey data (objective points) using a high-accuracy independent dataset (the "trusted" benchmarks). Assuming the errors are distributed normally, vertical accuracy at the 95-percent confidence level is computed from the RMSE (Wilson and Richards, 2006). The NSSDA vertical accuracy (Federal Geographic Data Committee, 1998) is computed using the following equation:

$$
R M S E_{z}=\sqrt{\frac{\sum_{i=1}^{n}\left(Z_{\text {data }_{i}}-Z_{\text {check }_{i}}\right)^{2}}{n}}
$$

where

$$
\begin{array}{cl}
R M S E_{z} & \text { is the vertical root mean square error, } \\
z_{\text {data }_{i}} & \text { is the vertical coordinate of the } i \text { th check point } \\
\text { in the dataset, }
\end{array}
$$

$i \quad$ is an integer from 1 to $n$, and

$n \quad$ is the number points being checked.

$$
A_{z}=1.960^{*} R M S E_{z}
$$

where

$$
\begin{aligned}
& A_{z} \quad \text { is the fundamental vertical accuracy } \\
& \text { calculated at the 95-percent confidence } \\
& \text { level. }
\end{aligned}
$$

For example, a Level II survey utilizing six trusted benchmarks will use equation 3 to sum the squared residuals resulting from observed and known benchmark vertical coordinates, divide by the number (six) of these trusted benchmarks used, and apply the square root to yield an accuracy at the 68-percent confidence level (or 1 sigma). To further express the accuracy at the 95-percent confidence level (or 2 sigma), the resulting accuracy is multiplied by 1.960 as identified in equation 4 .

\section{Metadata}

A concern with any type of GNSS survey is the storage of attributes behind the data collected during a campaign. It is important, and therefore recommended, that a sufficient record be documented to maintain the geodetic trail back to the datum (Henning, 2010). Benchmark recovery or establishment is often the foundation of a GNSS campaign and there are many attributes that need to be recorded (appendix 1).

For benchmark recovery, appendix 1 documents the following information necessary to record and archive:

- Name, date, state, county, latitude, longitude, datum, and elevation.

- USGS topographic 1:24,000 quadrangle map on which the benchmark resides.

- Benchmark station designation: a designation that typically does not match exactly with the stamping of most marks, and that may represent one or a combination of characteristics, such as the project, location, or date.

- Station Permanent Identifier (PID): a station identifier that typically matches what is stamped on the benchmark and consists of 2 upper case letters followed by 4 numbers (For NGS benchmarks only).

- Benchmark accuracy: each benchmark will have an accuracy designation. Vertical order benchmarks are usually noted by order 1, 2, or 3, and are further discussed in a document by the Federal Geodetic Control Committee (FGCC) titled "Geometric and Geodetic Accuracy Standards and Specifications for Using GPS Relative Positioning Techniques" (Hull, 1989). The benchmark may have some other localized accuracy identified through a specific project. 
- Recovery condition: the recovered condition; good condition, not located or disturbed.

- Monumented or non-monumented information: material of the benchmark, shape, description, and relief. An etching or photograph of the benchmark is recommended for documentation.

- Location information; GNSS observation suitability, land location in which benchmark resides, and any information pertaining to access or property owner.

- "To reach" narrative: description of a "leg-by-leg" distance and direction from major roadway intersection. This description should be detailed enough that the mark can be located without any other aids.

- Benchmark description and measurements: site sketch that provides some detail as to location supported by three measurements to permanent nearby objects. This box is used to address additional detail necessary to recover the benchmark.

- Reference measurements from station: distance and direction from three objects illustrated in adjacent sketch.

- A visibility-obstruction diagram used to describe any potential interference above a 10 degree plane.

For benchmark establishment, the form in appendix 1 may be used to document the following information necessary to record and archive:

- Name, date, state, county, latitude, longitude, datum, and elevation (to be derived).

- USGS topographic 1:24,000 quadrangle map on which the established mark resides.

- Established benchmark station designation: a designation may represent one or a combination of characteristics such as the project, location, or date.

- Established quality: Level I, II, III, or IV.

- Monumented or non-monumented information: constructed and installed material of benchmark, shape, relief, and annotated description.

- Location information: as described for recovered benchmarks.

- "To reach" narrative: as described for recovered benchmarks.

- Benchmark description and measurements: as described for recovered benchmarks.

- Reference measurements from station: as described for recovered benchmarks.
- Visibility-obstruction diagram as described for recovered benchmarks.

Along with benchmark recovery and establishment, a form is needed to document GNSS observations. Appendix 2 illustrates all necessary information to be documented for static data collection. This form may be used with the base station for single-base RTK solutions that are post processed; however, this form is not intended to document data collected by the rover during RT methods, so these data should be kept in a field notebook. Appendix 2 provides the user a script to ensure a well-documented static observation with definitions for the following entries listed below:

- Name, date, station designation, and station permanent identifier (PID) as previously defined for appendix 1.

- Interval of time in days and fractions of a day: corresponds to a Julian date recorded in the receiver and used when processing.

- Observation session times: time provided in Coordinated Universal Time (UTC) recorded in the receiver and used for processing.

- Collection interval and elevation mask: the collection interval is the interval in which the data are collected. As previously discussed for OPUS processing, intervals of $1,2,3,5,10,15$, and 30 are accepted. The elevation mask value is typically $10-15$ degrees.

- Datum, adjustment, and epoch: most commonly NAD 83 (CORS96), epoch 2002.0; however, a more common adjustment NAD 83 (NSRS 2007), epoch 2007.0 is available along with a new multi-year CORS adjustment NAD 83 (2011), epoch 2010 released the first day of January 2012. Other historic adjustments include NAD 83 (1986), NAD 83 (HARN), and NAD 83 (FBN-CBN).

- Other stations observed during session: this includes all active and passive (benchmark) stations while conducting an observation. This information is necessary for network surveys.

- Receiver and receiver antenna brand and model, and radio interference: this block identifies the receiver used and the available serial number. The serial number is commonly used to distinguish observation sessions when post processing. The antenna brand and model is also necessary to ensure the appropriate antenna calibration model for processing. Modern receivers generally are a combination thereof, including a receiver and built-in antenna. The presence of a radio interference source should be documented for avoidance and tracing problematic solutions. For dual-frequency receivers, the signal strength or power of the $\mathrm{L}_{2}$ signal is much lower than the $\mathrm{L}_{1}$ signal. It is not uncommon for the $\mathrm{L}_{2}$ signal to suffer interference. 
High wattage transmission lines and broadcast antennas are common suspects.

- The Antenna Reference Point (ARP) height: this block illustrates the correct way to determine an ARP and requires a measurement before and after the observation.

- Weather observations: this block requires any distinguishing weather features or anomalies that may impact an observation session. Generally, temperature, wind, precipitation, or storms are indicated. Any type of weather front should be documented here.

- Coordinate system: the system used during the survey, most commonly Universal Transverse Mercator (UTM) and State Plane.

- Notes: this block is used to describe any other detail related to the observation. This may include planning information or quality assurance surrounding the observation session. It may include information pertaining to processing or the intention thereof.

Appendixes 1 and 2 provide background information regarding the campaign, but do not ensure all of the metadata necessary to provide a historical description. As part of mission planning, a field book should be kept that adequately describes the "footprints" of the campaign. The following are additional considerations that should be documented, based on a GNSS approach:

Static Network Surveys

- Mission planning documentation.

- Sketch of the network, including benchmarks constrained, dispersion of objective points, spacing of the network, and baseline lengths.

- Field conditions: number of satellites observed, PDOP, local weather, RMS of the solution(s), benchmark residuals as a check, objective point residuals (50 percent double occupied).

- Multipath conditions: potential issues documented in a visibility diagram. Interference conditions such as power lines.

- Processing notes (usually defined in a project within a software program) that include the software and benchmarks used.

- Archival of the overall project file, raw data files, and scanned or electronic forms (appendixes 1 and 2).

Single-Base Static Surveys-OPUS-S and OPUS-RS

- Mission planning documentation

- Field conditions: baseline lengths, number of satellites observed, PDOP, local weather, RMS of the solution(s) (normalized RMS for OPUS-RS solutions), vertical peak-to-peak, observation redundancy residuals (if applicable).

- Multipath conditions: potential issues documented in a visibility diagram, Including interference conditions such as power lines.

- Archival of RINEX files, OPUS-S/RS solution reports, and scanned forms (appendixes 1 and 2).

Single-base RTK and RTN surveys (derived from Henning, 2009 and 2010)

- Mission planning documentation

- NSRS Alignment (RTN surveys exclusively); how was the network adjusted to CORS sites and what is the range of the positional difference between reference station coordinates and those coordinates after adoption as a NGS CORS site? An example is provided in table 6 .

- Localization: was there project localization to benchmarks? If so, which benchmarks were held and what were the source, quality, and reliability of these as constrained points? What were the best fit residuals on these benchmarks? If the project area was large (greater than $10 \mathrm{~km}$ ), was the area subdivided to ensure a quality localization?

- Benchmark checks: which benchmarks were checked? Did the benchmarks reveal an adequate spatial representation of the established objective points? What were the residuals?

- Field conditions: what was the number of satellites observed, PDOP, local weather, RMS of the solution(s), and observation redundancy residuals (10 percent objective points if applicable).

- Multipath conditions: potential issues documented in a visibility diagram, including interference conditions such as power lines.

- Communication: document resulting intermittent communications or interference, such as nearby high wattage transmission lines, broadcast antennas, or battery failure.

- Archival of the overall project file, raw data files, and scanned forms (appendixes 1 and 2).

\section{Database Storage}

There are several outlets available to USGS surveyors for publishing benchmarks that are established using GNSS surveying methods. These outlets include the standard method of benchmark publishing in the IDB through bluebooking (National Geodetic Survey 1994a, 1994b, and 2003), 
publishing in OPUS-DB (as described earlier under advancements in single-base OPUS), or publishing in a USGS database. For instance, the USGS database pertaining to the water mission area, GWSI, has been updated to include the addition of "SGPS" representing survey-grade GPS in the list of latitude and longitude methods, and Levels I-IV as options under latitude and longitude accuracy codes. Level I-IV is selectable once the surveying method is set to "SGPS." At the time of this writing, many USGS databases lack functionality for proper storage of appropriate GNSS metadata; therefore, users will likely continue to use in-office notes to document datum realization, mark description, location description, and other relevant information to relocate and utilize the benchmark.

\section{GNSS Campaign}

A survey campaign involving GNSS includes considerable planning and decisions that need to be made to ensure the most optimal approach while also ensuring quality and timeliness. A comprehensive summary is documented below for review and assurance of the content thus far.

\section{Objectives}

To begin planning a GNSS campaign, the objective of the survey should be addressed first, which includes:

- Size of the survey area.

- Number of objective points.

- Desired quality of the final elevations determined for each objective point.

If the survey area is large, a static or RTN survey will likely be most feasible. If the number of objective points is small, a static survey or high-quality RT survey could be performed. If there are a large number of objective points, the surveying method will be decided by what quality is required and how much field and processing time is feasible. The desired quality of the final elevations will strongly affect the type of survey that is feasible for the study. If elevations are used for topographic mapping only, then spending additional time locating high-order benchmarks with redundant observations on every point is not practical; however, if a difference in elevation of $0.08 \mathrm{~m}$ is significant to the survey, then additional time, including quality checks, should be included in the survey.

Evaluation of the quality requirements for a campaign is an important step before selecting the surveying approach. The initial questions that should be answered concern the quality that the elevations should represent. Are the elevations going to be recorded to the nearest centimeter or tens of centimeters? What difference in elevation is relevant to the study? Does a small elevation difference change the science or does a larger elevation difference change the science? After understanding what quality of elevation needs to be recorded and what elevation difference is relevant to the scientific questions being addressed, consideration of other sources of error, aside from the GNSS survey, will need to be evaluated as part of the final product (elevation).

\section{Benchmarks}

The second step in planning a campaign is to locate all available benchmarks. Information regarding quantity, order, and proximity of vertical benchmarks will contribute to a decision process regarding campaign quality. Benchmark information can be located by:

- Checking the NGS IDB

- Checking OPUS-DB

- Checking databases of other Federal, State, or local agencies

Caution should be exercised when using non-NGS benchmarks because datum (realizations) are likely different. Benchmarks used in any campaign need to be evaluated for prior GNSS data collection, stability, order, and station recovery notes. These attributes assure recovery and use in the field. The location of the benchmarks should also be considered when selecting those marks to recover and use in the campaign. Benchmarks should surround the project area if possible. Evaluation of benchmark geometry can be accomplished by plotting a topographic map or using other software or web utilities to provide a visual representation. The topographic map (or online mapping utilities such as Google Earth, 2011) may be used to illustrate areas with canopy, provide relief for optimal base station occupation and baseline development (radio communication) for objective points, and identify roadways for logistics. After benchmarks and objective points have been evaluated, and a survey approach determined, a decision tree (fig. 23) may be utilized to select the appropriate level quality of survey to fulfill the objective of the campaign.

\section{Decision Tree}

The decision tree should be used to assist in providing the quality of survey that might be possible with the current benchmarks available. GNSS users should incorporate the requirements of the survey, the decision tree, maps of the area, time for survey, personnel available, and equipment available to determine the final survey schedule.

\section{Reconnaissance}

Once the survey method, candidate benchmark recovery, and establishment locations have been determined, a reconnaissance trip to the project area is necessary. All maps and benchmark information should be taken on the reconnaissance trip to aid in benchmark recovery (including benchmark 


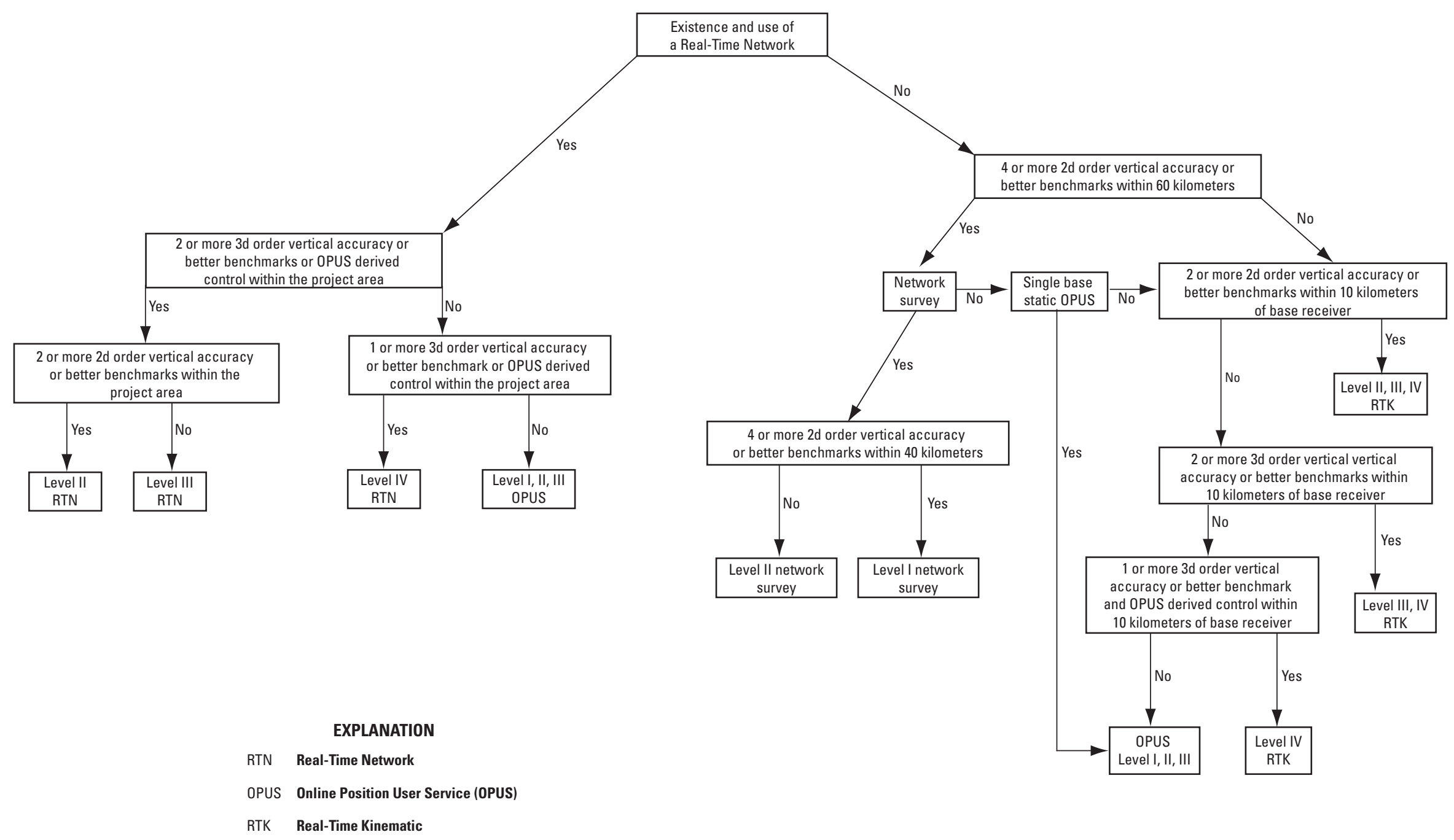

Figure 23. Decision tree for a level quality Global Navigation Satellite System (GNSS) survey approach. 
recovery and establishment forms in appendix 1). Access and logistics should be evaluated for benchmark recovery and establishment locations during the reconnaissance trip. This includes contacts with landowners regarding the type work being done and dates for the field effort. If several benchmarks are not recovered or are recovered in poor condition, the previous steps of finding benchmarks, reviewing the maps of benchmark locations, and working through the decision tree to determine the quality of survey should be performed.

\section{Field Preparedness}

With the survey method and benchmarks determined, the field schedule can be developed with knowledge of the number of GNSS receivers to be used and the staff available. The field schedule should document the overall length of time the effort will take and the dates accordingly. Additionally, the schedule should identify which benchmarks and objective points are to be surveyed each day, quality checks to be implemented each day, and the uncertainty those checks should meet. A detailed field schedule that includes individuals responsible for surveying each benchmark and objective point might be necessary if the presence of a large crew is expected. In addition, time should be allotted for benchmark construction if necessary.

Once survey dates have been established, mission planning for the dates and the location should be completed as described in the "Mission Planning and Error Sources" section. Additional preparations include the development of a contact list of the field crew and survey chief for campaign changes or problems that arise. An equipment checklist should be established and reviewed before deploying to the field. All equipment should be charged and calibration of equipment (such as bubble levels) should be completed. It is often necessary to maintain a survey truck in which conventional and fixed-height tripods can be stored and hauled safely. The truck should include all benchmark setting equipment (if needed) and backup equipment, such as cables, tripods, batteries, and battery chargers.

Field preparation also involves an evaluation and upload of the current geoid model, survey line files, background maps such as aerial photography, and control coordinates into the data collector. If projects are set up with these elements before the field survey, it is less likely that incorrect coordinates or other mistakes will be made. In addition, if there are file or point naming conventions that should be used during the survey, these should be determined and provided to each crew member for reference in the field.

If a campaign requires special equipment, such as rental of additional GNSS equipment, a radio repeater, or a RTK bridge, this equipment should be tested and the application subsequently planned. Possible setup locations of the radio repeater or RTK Bridge can be mapped for crew members to utilize during the survey.

\section{Survey}

During the campaign, the following practices should be followed:

- Take the time to make sufficient field notes.

- Follow the requirements for the level quality of surveying selected.

- Fill out all worksheets in the field (avoid waiting until you get back to the office).

- Document benchmarks, surroundings, and any important aspect of the campaign with pictures.

- Work systematically through troubleshooting because no GNSS campaign goes smoothly, even with planning; however, working logically through the problem and understanding the different sources of errors and flags the data collector is disseminating will provide the most effective assurance during the campaign.

- Download data daily (preferably in the evening) so no data are lost if a piece of equipment is damaged.

\section{Data Download, Analysis, Storage, and Documentation}

Upon completion of a survey campaign and return to the office, all equipment should be cleaned, organized, and properly stowed. Data collected on the receivers and the data collectors should be downloaded from the devices or field computer to the network (or at least backed up on a separate computer or hard drive). All data files should be reviewed and cross-checked with field notebooks and other recorded metadata to ensure that all expected files have been downloaded. If the data are in a form that can be evaluated (that is, setup files that show the base station location information entered, coordinate systems, checks recorded at all benchmarks, rover pole heights, antenna types, RINEX file time spans, and other details), they should be reviewed in each file when possible.

After the GNSS campaign, the results should be evaluated to determine if the desired survey quality has been met. Survey data, as well as all metadata, should be stored and documented according to guidelines provided in the "Metadata" section so that the information can be easily accessed in the future. It may be necessary to provide a summary report that describes the "footprints" of the campaign in the field, metadata, and tables that represent redundancy residuals for objective points and benchmarks. These data can be used collectively to provide a numerical representation of accuracy to what is held as "truth" (for example benchmarks). 


\section{Future Direction of GNSS}

It is difficult to assess the future of global positioning at the time of this writing. Many accepted approaches to GNSS campaigns may be changed beyond the next decade. The modernization of GPS in the United States began because once GPS reached a fully operation status, new demands were placed upon the system with the advance of technology. To date, many improvements have been made and many are still forthcoming. In addition to the advance of new ground stations as part of GPS modernization, additional satellite signals, such as $\mathrm{L}_{2} \mathrm{C}$, have been made available on the block IIR-M satellite series (U.S. Naval Observatory, 2010). This additional civilian signal permits faster signal acquisition, enhanced reliability, and a greater operating range (U.S. Department of Commerce, 2010). As reported by the U.S. Naval Observatory (USNO) at the time of this writing, another 11 satellites of the block II-F satellite series are to be launched in the future. This is the series carrying the $\mathrm{L}_{5}$ signal, a civilian-use signal developed primarily for aviation, and anticipated to have improved strength greater than the existing $\mathrm{L}_{1}$ and $\mathrm{L}_{2}$ signals. The first and second block II-F satellites were launched in May 2010 and July 2011, with the third scheduled for 2012. Many manufacturers have receivers on the market compatible with the $\mathrm{L}_{5}$ signal, which will generally permit better signal tracking, ambiguity resolution, and interference prevention. The increased signal strength of the new $\mathrm{L}_{5}$ signal will provide inherent multipath resistance for receivers that are designed with receiver-based multipath mitigation algorithms for increased SNR (Weill, 2003). In addition to the anticipated launch of 11 additional block II satellites, a block III series of satellites is subsequently anticipated with many improvements; specifically, increased signal power and signal integrity at the Earth's surface (Lazar, 2007).

The block III series is also anticipated to share a common civilian signal $\mathrm{L}_{1} \mathrm{C}$ with the Galileo system (Crews, 2008).

As well as the block I and II series of satellites, the block III series will progressively demonstrate a reduction in error associated with signals in space, such as satellite clocks, ephemeris data, and delays related to the ionosphere and troposphere (Crews, 2008).

At the time of this writing, the GLONASS constellation maintains 22 operational satellites and another 4 satellites are in maintenance (Federal Space Agency, 2011). Future plans for the Russian-based constellation are to achieve and maintain its Fully Operational Capability (FOC) of 24 healthy transmitting satellites. The current constellation transmits $\mathrm{L}_{1}$ and $\mathrm{L}_{2}$ signals. The GLONASS constellation has also been undergoing modernization with GLONASS-M and GLONASS-K satellites designed for stronger signal characteristics and a longer design life (Andrews Space \& Technology, 2001). As part of the modernization program, it is anticipated that the GLONASS-K satellite series will be transmitting $\mathrm{L}_{1}, \mathrm{~L}_{2}, \mathrm{~L}_{3}$, and $\mathrm{L}_{5}$ signals (Revnivykh, 2010).

Also at the time of this writing, the Galileo constellation maintains experimental satellites GIOVE-A and GIOVE-B. The constellation will reach a full constellation of 27 satellites with 3 satellites in reserve (Federal Space Agency, 2011). The Galileo constellation anticipates broadcasting 10 different signals including common signal broadcasts by other navigation systems $\mathrm{L}_{1}, \mathrm{~L}_{2}$, and $\mathrm{L}_{5}$ (Federal Space Agency, 2011).

Another constellation anticipated to be a part of an active GNSS is the Chinese Compass constellation. The Compass is a new system anticipated to reach an FOC of 35 satellites (Gibbons Media \& Research, 2010). At the time of this writing, the Chinese Compass constellation has received six successful launches (Xinhau News Agency, 2010) and various signals have been tracked to date.

As GNSS constellations are under development or modernization programs are initiated, manufacturers work with government agencies to ensure tracking by survey-grade receivers. It is presumptuous to forecast specifics that may arise from three or more frequencies received from several GNSS constellations at their FOC. According to Chen and others (2004), although the resolution of ambiguities and positioning performance is enhanced by the additional frequencies available, the RTN will still dominate in reducing atmospheric effects for RT positioning. Other studies are examining the potential for precise point positioning (PPP), which is a technique that utilizes measurements at the user's receiver, as opposed to a reference or base station. With the expansion of satellite constellations, increased observations may have a substantial effect on many of the greatest weaknesses of the GNSS technology: convergence time, accuracy, and integrity (Gisnath and Gao, 2009). With the expansion of the current GNSS and further algorithm development, PPP technology could emerge as a commensurate approach to RTK (Gisnath and Gao, 2009).

Another future aspect of GNSS and its application to vertical datum is the progression of the Gravity for the Redefinition of the American Vertical Datum (GRAV-D) project by the NGS. The specific goal of the project is to model and monitor the Earth's geoid (Smith, 2007). The effort entails a gravity survey of the entire United States and its holdings, which will produce a gravity-based vertical datum within $2 \mathrm{~cm}$ for much of the country (Smith, 2007). Gravity measurements are the foundation for development and modeling of the geoid. As discussed in the "Introduction" of this manual, the geoid permits the conversion of GNSS-produced ellipsoid heights to orthometric heights. It is these orthometric heights that are commonly referred to as "height above sea level." Completion of the anticipated effort is projected for the year 2021 (National Geodetic Survey, 2011b). 


\section{Selected References}

Andrews Space \& Technology, 2001, GLONASS-Summary: Andrews Space \& Technology, accessed February 21, 2011, at http://www.spaceandtech.com/spacedata/constellations/ glonass_consum.shtml.

Armstrong, M.L., 2010, OPUS Programs Suite Update presentation at the OGUG meeting, June 18, 2010, accessed March 17, 2011, at http://www.oregon.gov/ODOT/HWY/ GEOMETRONICS/ngs_advisor.shtml.

Ashtech Precision Products, 2001, Ashtech solutions user's guide: Santa Clara, Calif., Magellan Corporation, 170 p.

Brinker, R.C., and Minnick, R., 1995, The surveying handbook: Kluwer Academic Publishers, 924 p., accessed June 30, 2011, at http://books.google.com.

Burkard, R.K., 1985, Geodesy for the layman (5th ed): National Oceanic and Atmospheric Administration, 93p.

California Department of Transportation, 2006, Global Positioning System (GPS) survey specifications: California Department of Transportation, Surveys Manual, $42 \mathrm{p}$.

Carter, A., 2009, Understanding the Trimble Survey Controller Site Calibration Scale Factor, Trimble: EMEA Training Manager, February 23-25, 2009.

Chen, X., Vollath, U., Landau, H., and Sauer, K., 2004, Will Galileo/Modernized GPS Obsolete Network RTK?: Trimble Terrasat GmbH, Trimble White Paper, Trimble Navigation Limited, accessed February 21, 2011, at http://www. inavsoft.com/pdf/GALILEO-GPSL5-network-RTK.pdf.

Crews, M., 2008, Long-Term Future of GPS: San Diego, Calif., Institute of Navigation (ION), accessed February 21, 2011, at $h t t p: / / w w w . p n t . g o v / p u b l i c / 2008 / 2008-01$ NTM/crews.pdf.

Cunningham, W.L., and Schalk, C.W., comps., 2011, Groundwater technical procedures of the U.S. Geological Survey: U.S. Geological Survey Techniques and Methods, book 1, chap. A1, $151 \mathrm{p}$.

Eckl, M.C., Snay, R.A., Soler, T., Cline, M.W., and Mader, G.L., 2001, Accuracy of GPS-derived relativepositions as a function of interstation distance and observingsession duration: Journal of Geodesy, v. 75, p. 633-640.

Federal Geographic Data Committee, 1998, Geospatial positioning accuracy standards, part 3-National standard for spatial data accuracy: Federal Geographic Data Committee, U.S. Geological Survey, 28 p. (Also available at $h t t p: / / w w w$. fgdc.gov/standards/projects/FGDC-standards-projects/ accuracy/part3/chapter 3 .)
Federal Space Agency, 2011, GLONASS constellation status: Information-Analytical Centre, accessed February 21, 2011, at $h t t p: / / w w w . g l o n a s s-i a n c . r s a . r u / e n /$.

Gibbons Media \& Research, 2010, China begins broadcasts on latest compass GNSS GEO Satellite: Inside GNSS, Gibbons Media \& Research, accessed February 21, 2011, at http:// www.insidegnss.com/node/2134.

Gisnath, S., and Gao, Y., 2009, "Innovation: Precise Point Positioning," Algorithms \& Methods: GPS World, 2011 Questex Media Group, accessed February 21, 2011, at http://www.gpsworld.com/gnss-system/algorithms-methods/ innovation-precise-point-positioning-7040?page_id $=1$.

GlobalSecurity.org, 2010, accessed February 9, 2010, at http:// www.globalsecurity.org/military/library/budget/fy1999/ dot-e/airforce/99gps.html.

Google, 2011, Google Earth-2011 Europa Technologies: U.S. Department of State Geographer, accessed August 9, 2011, at http://www.google.com/earth/index.html.

Henning, W.E., 2007, Real time GPS Networks (RTN) and their implications with Geographic Information Systems (GIS), Strategic Integration of Surveying Services, FIG Working Week 2007, Hong Kong SAR, China, 13-17 May 2007.

Henning,W.E., 2009, User Guidelines for Real Time GNSS Networks: National Geodetic Survey, 68 p.

Henning,W.E., 2010, User Guidelines for Single Base Real Time GNSS Positioning: National Geodetic Survey, 151 p.

Hull, W.V, 1989, Geometric geodetic accuracy standards and specifications for using GPS relative positioning techniques: Federal Geodetic Control Committee, ver. 5.0.

Jones, B.A., and Kelly, K.M., 2007, Real time GPS Networks (RTN) and their implications with Geographic Information Systems (GIS), Strategic Integration of Surveying Services, FIG Working Week 2007, Hong Kong SAR, China, 13-17 May 2007.

Kennedy, E.J., 1990, Levels at streamflow gaging stations: U.S. Geological Survey Techniques of Water-Resources Investigations, book 3, chap. A19, 31 p., (Also available online at http://pubs.usgs.gov/twri/twri3-A19/pdf/twri_3A19_a.pdf.)

Kenney, T.A., 2010, Levels at gaging stations: U.S. Geological Survey Techniques and Methods, book 3, chap. A19, $60 \mathrm{p}$.

Kowoma.de, 2009, GPS-System-Transmitted signals, accessed September 28, 2010, at http://www.kowoma.de/en/ gps/index.htm. 
Lapine, L.A., 2010, Another coordinate system this time without survey marks: The American Surveyor, v. 7, no. 8, 4 p., accessed March 1, 2011, at http://www.amerisurv.com/PDF/ TheAmericanSurveyor Lapine-AnotherCoordinateSystem Vol7No8.pdf.

Lazar, S., 2007, Modernization and the move to GPS III: The Aerospace Corporation, accessed February 21, 2011, at http://www.hjgz.net/hhyq/zyq/ wenxian\%5CModernization\%20and\%20the\%20Move\%20 to\%20GPS\%20III.swf.

Mader, G.L., 1999, GPS antenna calibration at the National Geodetic Survey: Silver Springs, Md., National Geodetic Survey, accessed March 23, 2011, at http://www.ngs.noaa. gov/ANTCAL/images/summary.html.

Mader, G.L., 2010, GPS antenna calibration: National Geodetic Survey, accessed March 23, 2011, at http://www.ngs. noaa.gov/ANTCAL/.

Martin, D., 2007, Geodetic connections-OPUS rapid static: The American Surveyor, v. 4, no. 3, p. 44-48.

National Geodetic Survey, 1994a, Input formats and specifications of the National Geodetic Survey data base, Volume II, Vertical control data: Silver Springs, Md., National Geodetic Survey, 6 p.

National Geodetic Survey, 1994b, Input formats and specifications of the National Geodetic Survey Data Base: Volume III, Gravity Control Data; National Geodetic Survey, Silver Springs, Md., 56 p.

National Geodetic Survey, 2001a, Positioning accuracy standards presentation at the North Carolina Society of Surveyors, Proceedings: New Bern, N.C., accessed March 23, 2011, at http://www.ngs.noaa.gov/PC_PROD/WorkShops/ PPT/Standards-NewBern.ppt.

National Geodetic Survey, 2001b, Geodetic glossary: Silver Springs, Md., National Geodetic Survey, accessed March 23, 2011, at http://www.ngs.noaa.gov/CORS-Proxy/ Glossary/xml/NGS_Glossary.xml. [Last updated July 3, 2009.]

National Geodetic Survey, 2001c, What is the Geoid?: Silver Springs, Md., National Geodetic Survey, accessed June 12, 2012, at http://www.ngs.noaa.gov/GEOID/geoid_def.html. [Last updated January 30, 2001.]

National Geodetic Survey, 2003, Input formats and specifications of the National Geodetic Survey Data Base, Volume I, Horizontal Control Data: Silver Springs, Md., National Geodetic Survey, 6 p.

National Geodetic Survey, 2010, FAQ: Frequently asked questions about the National Geodetic Survey, accessed April 20, 2010, at http://www.ngs.noaa.gov/faq. shtml\#WhyVD29VD88.
National Geodetic Survey, 2010a, OPUS-RS: National Geodetic Survey, accessed March 17, 2011, at http://www.ngs. noaa.gov/OPUSI/Plots/Gmap/OPUSRS_sigmap.shtml.

National Geodetic Survey, 2010b, Datasheet Retrieval Page, accessed August 19, 2010, at http://www.ngs.noaa.gov/cgibin/datasheet.prl.

National Geodetic Survey, 2010c, CORS: Continuously Operating Reference Station (CORS): National Geodetic Survey, accessed September 21, 2012, at http://www.ngs.noaa.gov/ CORS/.

National Geodetic Survey, 2010d, Online Position Users Service (OPUS): National Geodetic Survey, accessed September 28, 2010, at http://www.ngs.noaa.gov/OPUS/about.html.

National Geodetic Survey, 2011a, OPUS: Online Positioning User Service (OPUS): National Geodetic Survey, accessed February 8, 2011, at http://geodesy.noaa.gov/OPUS/.

National Geodetic Survey, 2011b, Gravity for the Redefinition of the American Vertical Datum (GRAV-D): National Geodetic Survey, accessed February 21, 2011, at http://www. ngs.noaa.gov/GRAV-DAV-D/.

National Geodetic Survey, 2012, NGS Geodetic Advisors: National Geodetic Survey, accessed June 12, 2012, at http:// www.ngs.noaa.gov/ADVISORS/AdvisorsIndex.shtml.

National Geodetic Survey, 2012b, OPUS-RS Map: National Geodetic Survey, accessed June 12, 2012, at http://www.ngs. noaa.gov/OPUSI/Plots/Gmap/OPUSRS_sigmap.shtml.

National Geodetic Survey, 2012c, National Adjustment of 2011 (NA2011); NAD 83(2011) epoch 2010.00 coordinates on passive control, accessed August 14, 2012, at http://www. ngs.noaa.gov/web/surveys/NA2011/.

National Digital Elevation Program, 2004, Guidelines for Digital Elevation Data: U.S. Department of the Interior, U.S. Geological Survey, ver. 1.0.

National Security Council, 1996, Office of Science and Technology Policy-U.S. Global Positioning System Policy: National Security Council accessed February 9, 2010, at http://clinton4.nara.gov/textonly/WH/EOP/OSTP/html/gpsfactsheet.html.

National Weather Service, 2005, Geophysical alert message description: National Oceanic and Atmospheric Administration, accessed August 30, 2010, at http://www.swpc.noaa. gov/Data/info/WWVdoc.html\#description.

National Weather Service, 2009, Solar cycle progression: National Oceanic and Atmospheric Administration, accessed August 31, 2010, at http://www.swpc.noaa.gov/ SolarCycle. 
NavtechGPS, 2012, Galileo Equipment, accessed August 31, 2011, at https://www.navtechgps.com/Shop/ equipment/galileo_equipment/.

Office of Science and Technology Policy (OSTP), National Security Council, 1996, Press Release-U.S. Global Positioning System Policy, accessed February 9, 2010, at http://clinton4.nara.gov/textonly/WH/EOP/OSTP/html/gpsfactsheet.html.

OPUS-Projects Team, 2011, OPUS-Projects: National Geodetic Survey, accessed March 17, 2011, athttp://www.ngs. noaa.gov/OPUSI/OpusProjects.html.

Pacific Crest Corporation, 2009, Application note-Resolving radio range issues: Pacific Crest Corporation, accessed March 17, 2011, at http://paccrst.com/library/AppNote_ ResolvingRadioRangeIssuues90409.pdf.

Parker, D.A.S., 1994, A differential global positioning system for flight inspection of radio navigation aids: Parker Hannifin Corporation, Gulf Electronics, [Ref.: TP0029], accessed March 17, 2011, at http://webone.novatel.ca/assets/ Documents/Papers/File29.pdf.

Revnivykh, S., 2010, GLONASS status and progress: Civil Global Positioning Service Interface Committee (CGSIC), accessed September 21, 2010, at http://www.navcen.uscg. gov/pdf/cgsicMeetings $/ 50 / \% 5 B 3 \% 5 D C G S I C \_G L O N A S S$ Revnivykh_20_09_2010.pdf.

Rizos, C., 200, Network RTK Research and ImplementationA Geodetic Perspective: Sydney, Australia, School of Surveying and Spatial Information Systems, University of New South Wales, 7 p.

Roman, D.R., Wang, Y.M., Saleh, J., Xiaopeng, L., 2009, A gravimetric geoid model for the United States-The development and evaluation of USGG2009: NOAA/National Geodetic Survey, accessed February 21, 2010, at http:// www.wfps.org/files/AMUW09/Handouts/Friday\%20-\%20 Wang\%20(Roman)(75).pdf.

Schenewerk, Mark, 2011, New Developments for OPUS presentation, in Annual Rocky Mountain Surveyors Summit, 1st, Arvada, Colo., 2011, accessed March 23, 2011, at http:// www.ngs.noaa.gov/web/science_edu/presentations_archive/.

Schwarz, C.R., Snay, R.A., and Tomás, S., 2009, Accuracy assessment of the National Geodetic Survey's OPUS-RS utility: GPS Solutions, v. 13, no. 2, p. 119-132.

Skeen, J., 2005, TXDOT GPS User's Manual: Texas Department of Transportation, accessed February 9, 2010, at https://www.e-education.psu.edu/files/geog488/downloads/ gps.pdf.
Smith, D., 2007, The GRAV-D Project, Gravity for the Redefinition of the American Vertical Datum: NOAA/National Geodetic Survey, accessed February 21, 2011, at http:// www.ngs.noaa.gov/GRAV-D/pubs/GRAV-D_v2007_12_19. $p d f$.

Snay, R., 2009, OPUS presentation, in Great Lakes Regional Height Modernization Forum, Lansing, Mich., 2009, accessed February 9, 2011, at http://www.ngs.noaa.gov/ CORS/presentations.shtml.

Soler, T., and Marshall, J., 2003, A note on frame transformations with applications to geodetic datums: Silver Spring, Md., National Geodetic Survey, N/NGS22, \#8825. 21 p.

Stone, W., 2011, National Geodetic Survey Programs and Geodetic Tools, in conference of the California Land Surveyors Association and the Nevada Association of Land Surveyors, Las Vegas, Nev., 2011, accessed February 9, 2011, at http:// www.ngs.noaa.gov/web/science_edu/presentations_archivel clsa-nals_2011_stone-ngs.pptx.

Trimble, 1992-2002, Convert to RINEX, 1992-2002, Dat2Rin DLL, ver. 3.50, Copyright 1992-2002: Trimble Navigation.

Trimble, 2002, Trimble Geomatics Office User Guide: Trimble, v. 1.6, 46741-20 ENG, chap.8.

Trimble, 2011, GPS Tutorial: Trimble Navigation Limited, accessed February 7, 2011, at http://www.trimble.com/ gps_tutorial/.

Trimble Business Center, 2011, ver. 2.50: Trimble Navigation Limited.

U.S. Department of Commerce, 2010, GPS modernization: NOAA and Office of Space Commerce, accessed April 20, 2010, at http://www.space.commerce.gov/gps/ modernization.shtml.

U.S. Department of Homeland Security, United States Coast Guard, 2010, Navigation Center: accessed September 7, 2010, at http://www.navcen.uscg.gov/index. php? pageName $=$ nanuAbbreviations.

U.S. Geological Survey, 1966, Topographic instructions of the United States Geological Survey_Leveling: U.S. Geological Survey Topographical Instructions, book 2, chaps. 2E1$2 \mathrm{E} 5,63 \mathrm{p}$.

U.S. Geological Survey, 2004, User's manual for the National Water Information System of the U.S. Geological SurveyGround-water Site-Inventory System: U.S. Geological Survey Open-File Report 2004-1238, ver. 4.3. 
U.S. Geological Survey, 2010, U.S. Geological Survey National Geospatial Program Lidar Guidelines and Base Specification, ver. 13, accessed August 14, 2012, at http:// lidar.cr.usgs.gov/USGSNGP\%20Lidar\%20Guidelines\%20 and\%20Base\%20Specification\%20v13(ILMF).pdf.

U.S. Geological Survey, 2012, Start with Science, accessed June 12, 2012, at http://www.usgs.gov/start_with_science/.

U.S. Geological Survey, 2012, Ask USGS, accessed June 12, 2012, at http://ask.usgs.gov/.

U.S. Naval Observatory, 2010, Time services department: Department of the Navy, accessed February 10, 2011, at ftp://tycho.usno.navy.mil/pub/gps/gpsb2.txt.

Weill, L.R., 2003, Multipath mitigation-How good can it get with new signals?: GPS World, Questex Media Group LLC., accessed September 3, 2010, at http://www.gpsworld. com/gps/multipath-mitigation-812?page_id $=1$.

Weston, N., and Ray, J., 2011, GPS positioning performance from algorithm advances in the network version of OPUS: Geophysical Research Abstracts, v. 13, EGU20113901, 2011, EGU General Assembly 2011, accessed August 4, 2011, at http://meetingorganizer.copernicus.org/ EGU2011/EGU2011-3901.pdf.
Weston, N.D., Mader, G.L., and Soler, Tomás, 2007, OPUSProjects-A Web-based application to administer and process multi-day GPS campaign data: National Geodetic Survey, accessed March 19, 2011, at http://www.ngs.noaa. gov/CORS/Articles/NeilFIG2007.pdf.

Wilson, G.L., and Richards, J.M., 2006, Procedural documentation and accuracy assessment of bathymetric maps and area/capacity tables for small reservoirs: U.S. Geological Survey Scientific Investigations Report 2006-5208, 24 p.

Xinhau News Agency, 2010, BeiDou navigation system covers Asia-Pacific region till 2012: accessed February 21, 2011, at http://news.xinhuanet.com/mil/2010-03/03/ content_13087844.htm.

Zilkoski, D.B., D’Onofrio, J.D., and Frakes, S.J., 1997, NOAA Technical Memorandum NOS NGS-58 - Guidelines for establishing GPS-derived elliposid heights (Standards: $2 \mathrm{~cm}$ and $5 \mathrm{~cm}$ ), ver. 4.3: Silver Springs, Md., National Geodetic Survey, $11 \mathrm{p}$.

Zilkoski, D.B., Carlson, E.E., and Smith, C.L., 2008, NOAA Technical Memorandum NOS NGS-59-Guidelines for establishing GPS-derived orthometric heights: Silver Springs, Md., National Geodetic Survey, 15 p. 



\section{Glossary}

A

accuracy The degree to which measurements derived from static or real-time (RT) positioning represent "truth." Trusted monuments, as compared with Global Navigation Satellite System (GNSS) positioning, are often used to evaluate accuracy during a campaign.

active stations (active control or monumentation) A Global Navigation Satellite System (GNSS) receiver and receiver antenna in a fixed location that is continually operating and collecting data. Continually Operating Reference Stations (CORS) are active control stations.

adjustment Processing of a value or data set to provide a more precise or accurate result based on the inclusion of control data using the process of least squares. Adjustments are used in network surveys involving static data collection.

algorithm A procedure or set of programmed instructions for resolving a mathematical problem to achieve an end result.

almanac A data file that contains the approximate positions for all satellites in orbit and the health of each satellite. This data file is transmitted every 12.5 minutes.

ambiguity resolution Carrier phase measurements are made in relation to a wavelength of the $\mathrm{L}_{1}$ or $\mathrm{L}_{2}$ carrier waves. When a receiver first locks on the wavelength of these carrier waves, the wavelength is a partial, and the number of whole wavelengths that precede that partial wavelength are unknown. To correctly calculate the distance the receiver is from the satellite, these preceding wavelengths must be known. The process used to determine the value for the ambiguities is known as "ambiguity resolution."

antenna (radio GNSS) Radio antenna used to facilitate broadcasted differential correction information from a Global Navigation Satellite System (GNSS) base receiver to a rover receiver. antenna (receiver GNSS) Small antenna elements contained within Global Navigation Satellite System (GNSS) receivers that receive and sometimes amplify an L-Band signal.

Antenna Reference Point (ARP) A point on the exterior of the receiver antenna, usually the bottom of the receiver antenna mount, to which the National Geodetic Survey references the antenna phase center position.

\section{B}

baseline A computed three-dimensional vector for a pair of stations for which simultaneous Global Navigation Satellite System (GNSS) data have been collected. Baselines are developed between a single-base RealTime Kinematic (RTK) base station and rover, or a Real-Time Network (RTN) reference station and rover. Baselines are also developed between static data-collecting receivers within a network survey.

blunder A gross error that prevents the desired position uncertainty from being achieved. As opposed to systematic or random errors, blunders are less discrete and are commonly the result of incorrect receiver antenna height or receiver antenna type entries, or collecting data during real-time (RT) positioning before the solution becomes initialized.

\section{C}

carrier phase signal The carrier frequency signal for timing measurements. The carrier frequency is high, and therefore wave cycles are smaller and can be more precisely defined than pseudo random code cycles; however, because the carrier frequency cycles are uniform, it is difficult to distinguish one cycle from another when assessing the number of cycles needed for measurement timing. Essentially, the pseudo random code is used to get a close estimate of travel time and the carrier signal is then used to refine the measurement. 
code phase signal The code frequency signal for timing measurements. The code frequency is lower than carrier frequency, and wave cycles are larger and more difficult to align than carrier cycles. Although receiver designers have developed ways to ensure signals are nearly identical in phase, cycle widths translate to substantial error. Therefore, pseudo random codes are used initially to get close estimates of travel time, which are further refined using the carrier signal.

Compact Measurement Record (CMR+) A data format used to frame packets of information and message types for $\mathrm{L}_{1}$ and $\mathrm{L}_{2}$ carrier phase and pseudo-range data, as well as reference (base) station location and description messages.

constellation A specific set of satellites used in calculating a position or all satellites visible to a Global Navigation Satellite System (GNSS) receiver at one time.

control point A benchmark representing assigned coordinates by terrestrial or satellite surveying techniques.

control segment A monitoring station used in conjunction with other stations to observe satellites and allow the calculation of precise orbits and ephemeris data.

D

data collector Also known as a data logger or data recorder. A ruggedized handheld portable data-entry computer used to store realtime (RT) positioning data. Data collectors facilitate localizations, change in units and datum, satellite observations, elevation masks, and coordinate geometry applications.

datum In geodetic terms, the datum is defined by its reference surface, an origin, an orientation, gravity, and a scale. The North American Datum 1983 (NAD 83) is defined by the Geodetic Reference System of 1980 (GRS 80) ellipsoid at an origin near the center of the mass of the Earth with axes oriented through the pose, equator, and at right angles, with a scale unit based on the international meter. The realization of this datum is through a reference, such as monumentation on the ground or Global Navigation Satellite System (GNSS) satellites with the ground control segment.
Dilution of Precision (DOP) An indicator of satellite geometry quality for a unique constellation. Poor satellite geometry leads to poor Dilution of Precision (DOP), triangulation, and location estimation. A low Dilution of Precision (DOP) value represents a better positional precision because of wide angular separation between the satellites used to calculate a terrestrial position.

dual-frequency Instrumentation that can make measurements on both L-Band frequencies, for example, $\mathrm{L}_{1}$ and $\mathrm{L}_{2}$, either pseudorange or carrier phase measurements.

\section{E}

elevation mask The minimum acceptable angle above the horizon that will minimize the greatest amount of noise because of atmospheric delay and refraction or potential multipath conditions.

ellipsoid height The height above or below a mathematically-defined surface or ellipsoid [for example, Geographic Reference System 1980 (GRS 80) or World Geodetic System 1984 (WGS 84)] that provides a representation of the Earth, flattened slightly at the poles, and bulging somewhat at the equator. The height coordinate determined by a Global Navigation Satellite System (GNSS) observation is related to the surface of the ellipsoid, typically WGS 84.

ephemeris A data file providing a particular satellite's position and velocity at any instant in time. Broadcast ephemeris represents a prediction of satellite position and velocity suitable for real-time (RT) positioning whereas precise ephemeris are post-processed values regarding satellite position and velocity suitable for static positioning.

epoch An instant in time. For real-time (RT) positioning, measurements are made at a given interval or epoch rate. An epoch may also represent a reference frame noted by a particular year. For example, North American Datum 1983 (NAD 83) Continually Operating Reference Station 1996 (CORS96), epoch 2002.0, or more recently from the multi-year CORS data [NAD 83 (2011) epoch 2010.00]. 
error ellipse An error estimate that results during an adjustment in a network survey. The error ellipse represents the geometric accuracy and alignment of a point, and is normally plotted at the 95-percent confidence level.

\section{$\mathbf{F}$}

\section{G}

geodetic survey Surveys performed for the establishment of control networks, which are the basis for accurate positioning and navigation. These surveys account for refraction, curvature of the earth, atmospheric conditions, and gravity, as opposed to "plane" surveys that generally ignore these considerations.

geoid The equipotential surface of the earth that most closely approximates global mean seal level. Refined as a hybrid model developed by gravimetric geoids (defined from gravity and terrain data) and separation distances between GNSS-derived ellipsoid heights and leveled benchmarks. This model is used to convert North American Datum 1983 (NAD 83) ellipsoid heights to North American Vertical Datum 1988 (NAVD 88) orthometric heights.

geoid height The separation distance between the reference ellipsoid Geographic Reference System 1980 (GRS 80) and the hybrid geoid model, for example GEOID 09. The combination of the North American Datum 1983 (NAD 83) ellipsoid height from Global Navigation Satellite System (GNSS) observations and this value enables a North American Vertical Datum 1988 (NAVD 88) orthometric height to be produced. The geoid height is positive away from the Earth center and negative towards it.

\section{Global Navigation Satellite System} (GNSS) A system of satellites providing autonomous geo-spatial positioning with global coverage.

\section{H}

\section{I}

International Terrestrial Reference Frame (ITRF) Realizations of the ITRS for a particular epoch in time, consisting of a set of three-dimensional coordinates and velocities for hundreds of geodetic stations around the world. Examples of reference frames as fol- lows: ITRF94, ITRF96, ITRF97, ITRF2000, ITRF2005, and ITRF2008.

International Terrestrial Reference System (ITRS) The most precise, geocentric, and globally-defined coordinate system or datum of the Earth. This system is managed by the International Earth Rotation and Reference System Service (IERS) located in Frankfurt, Germany.

ionosphere A band of the atmosphere extending from 30 to 600 miles above the earth that is ionized by solar radiation. This band of the atmosphere affects the behavior of radio waves.

\section{J}

K

\section{L}

L-Band The group of radio frequencies extending from $390 \mathrm{MHz}$ to $1560 \mathrm{MHz}$. The carrier frequencies $\mathrm{L}_{1}$ and $\mathrm{L}_{2}$ are in the L-Band.

$\mathbf{L}_{1}$ frequency The $1575.42 \mathrm{MHz}$ carrier frequency that contains the coarse/acquisition (C/A)-Code available to the general public, the encrypted P-Code for military access only, and the Navigation Message, which enables the determination of the time of transmission and satellite position at this time.

$\mathbf{L}_{2}$ frequency The $1227.60 \mathrm{MHz}$ carrier frequency that contains the encrypted P-Code for military access, and the Navigation Message, which enables the determination of the time of transmission and satellite position at this time. A combination of $\mathrm{L}_{1}$ and $\mathrm{L}_{2}$ permits the correction of Ionospheric delay. Dual-frequency GNSS receivers intended for surveying applications can produce $\mathrm{L}_{2}$ measurements using proprietary signal processing techniques.

$L_{1} \mathbf{C}$ frequency A future civilian-use signal to be broadcast on the same $\mathrm{L}_{1}$ frequency $(1575.42 \mathrm{MHz})$ that will be available with a future block III satellite launch. This signal is included as part of a modernization to the existing $\mathrm{L}_{1}$ signal to enable interoperability between GPS and international satellite navigation systems. Additionally, the signal features a waveform designed to improve mobile reception in cities and other challenging environments. 
$L_{2} \mathbf{C}$ frequency A modernized civilian-use signal broadcast on the same $\mathrm{L}_{2}$ frequency $(1227.60 \mathrm{MHz})$ transmitted by all block IIR-M satellites and later designs. When combined with the traditional $\mathrm{L}_{1}$ signal, $\mathrm{L}_{2} \mathrm{C}$ enables ionospheric correction, faster signal acquisition, enhanced reliability, and greater operating range. $\mathrm{L}_{2} \mathrm{C}$ operates at a higher power level than the traditional $\mathrm{L}_{1}$ signal, which promotes better tracking with surrounding obstruction.

$L_{5}$ frequency A civilian-use signal broadcast from the block II-F satellite launch. This 1575.42 MHz carrier frequency signal is better known as a "safety-of-life" signal with higher power and greater bandwidth developed for the aviation industry.

latency The age or time lapse in corrections used in real-time (RT) positioning. The longer the time lapse between the corrections, the less accurate they become at the rover.

least squares adjustment An adjustment in which the sum of the squares of the differences between given and changed positions are minimized. An adjustment that will tie all points together into a "best fit" network with the least amount of positional change.

localization (site calibration) A vertical shift applied to match a single elevation or planar surface. The user should assure trusted benchmarks are used to apply the vertical shift.

M

minimally constrained adjustment (inner adjustment) An adjustment in which the number of independent constraints are minimal, typically with just one position held fixed. This is done to evaluate the quality of baselines and detect other blunders in the network through residuals and adjusted observables without the distortion from several constraining positions.

monumented benchmarks Monumented benchmarks consist of a tablet with identifying information surrounding a stamped center point. These marks are located as a standard metal tablet, disk, cap, or steel rods used to describe the elevation. These tablets are commonly set in concrete, stone posts, firm rock outcroppings, masonry structures, and buildings. FENO markers are also considered monumented benchmarks. multipath Interference caused by reflected Global Navigation Satellite System (GNSS) signals arriving at the receiver, typically as a result of nearby structures or other reflective surfaces. The delayed signal causes a longer distance to the satellite. Multipath may be mitigated by proper antenna design, placement, and special filtering algorithms within GNSS receivers in static observations, but not for shorter times involved in real-time (RT) positioning. Reflective surfaces such as large buildings or structures, signboards, chain-link fence, and even mountains and water bodies may be suspect causes of potential multipath.

\section{N}

NAD 83 The North American Datum of 1983. The official national horizontal datum for the United States depicted as a threedimensional datum with coordinates of points expressed in latitude, longitude, and ellipsoid height. The NAD 83 origin is near the center of mass of the Earth.

\section{National Spatial Reference System}

(NSRS) The National Spatial Reference System is a consistent national coordinate system that specifies latitude, longitude, height, scale, gravity, and orientation throughout the Nation, as well as how these values change with time. The NSRS consists of National Continuously Operating Reference Stations (CORS), a network of permanent benchmarks, and a set of accurate models describing geophysical processes affecting spatial measurements.

NAVD 88 The North American Vertical Datum of 1988. Established in 1991 and referenced to the International Great Lakes Datum of 1985, local mean sea level height at Rimouski, Quebec, Canada.

navigation message Message broadcast every 12.5 minutes modulated on the $\mathrm{L}_{1}$ and $\mathrm{L}_{2}$ Global Navigation Satellite System (GNSS) signal containing the broadcast ephemeris, satellite clock bias correction parameters, constellation almanac information, and satellite health.

network survey Process by which individual static data-collecting receivers are occupied over benchmarks (may be augmented by active control stations) and arranged in a network that encompasses a project area where objective points will be established. 
noise An interfering signal that tends to mask the desired signal at the receiver. Causes stem from space and atmospheric phenomena, anthropogenic phenomena, or circuitry within the receiver.

non-monumented benchmarks Nonmonumented benchmarks may be considered semi-permanent monumentation that consists of chiseled squares; crosses or circles on concrete or masonry structures; bolt heads in steel, concrete, or masonry structures; and metal pins or magnetic (mag) nails in concrete or asphalt. Non-monumented benchmarks are simply a mark with no identifying information.

\section{0}

objective point The established point in a Global Navigation Satellite System (GNSS) survey, and considered to be a foresight in terrestrial surveying.

Online Position User Service (OPUS) A software service by the National Geodetic Survey providing access to the NSRS to derive coordinates from the CORS network.

orthometric height The height of a point on the Earth's surface, measured as a distance along a curved plumb line and normal to gravity from the reference surface to that point. Heights above or below that datum can be obtained through Global Navigation Satellite System (GNSS) methods by using the current hybrid geoid model and North American Datum 1983 (NAD 83) ellipsoid heights.

\section{P}

passive stations ( benchmarks) Referred to as a traditional ground station, such as a benchmark. Passive stations are those that can be occupied by survey equipment.

phase center The apparent center of signal reception at an antenna in a Global Navigation Satellite System (GNSS) receiver. The electrical phase center of an antenna is not constant, but is dependent upon the observation angle and azimuth to the satellite. The $\mathrm{L}_{1}$ and $\mathrm{L}_{2}$ phase centers are at different locations.

position The three-dimensional coordinate of a point, usually given in the form of latitude, longitude, and ellipsoid height. An estimate of error is often given with a position. post-processed GNSS Implies that the base and rover receivers had no communication link between them. Each receiver records satellite observations independently to allow processing of observables at a later time. Processing is facilitated by software.

precision The degree of repeatability that measurements of the same quantity display. A description of the quality of the data with respect to random errors. Precision is traditionally measured using standard deviation and may be thought of as the spread of the positional error.

pseudo-range A distance measurement based on the alignment of a satellite's time-tagged transmitted code and the local receiver's generated reference code (for that particular satellite) that has not been corrected for clock bias. A pseudo-range measurement is a distance measurement biased by a time error.

\section{$\mathbf{0}$}

R

Radio Technical Commission for Maritime Services (RTCM) Within a receiver, this standard message type is able to decode and apply differential corrections to its raw data to generate an error corrected coordinate.

range The distance between two points, such as a satellite and Global Navigation Satellite System (GNSS) receiver, or a base station and rover receiver in regard to radio communication.

Real-Time Kinematic, single-base (RTK) A traditional relative positioning procedure whereby observables and corrections for each $\mathrm{L}_{1}$ and $\mathrm{L}_{2}$ signal to each common satellite are transmitting in real time from a base station to the user's rover receiver. The rover receiver processes the data in real time. Centimeterlevel accuracy is achieved without any post processing.

realization A physical, usable manifestation of a particular datum. Realizations or alignments are usually performed on benchmarks with published coordinates, as located in the National Geodetic Survey Integrated Database (NGS IDB), or by locally set monuments; however, active monumentation can also serve as the basis for a realization. 
Real-Time Network A statewide network of continuously operating reference stations that are municipally, state, or privately owned. A centralized server is used to facilitate qualityassurance checks, network modeling, estimation of systematic errors, and calculation of corrected data that is submitted back to the end user at the rover position. The network operates by use of cellular communication, which excludes the requirement for a traditional base station to be used in the field.

receiver A device to track L-band satellite signals, the distance of the satellites, and employ the mathematical principal of trilateration to yield location.

reference station (base station) A ground station at a known or autonomous location used to derive differential corrections. The reference station receiver tracks all satellites in view, corrects pseudo-range errors, and then transmits the corrections with the carrierphase observables to the rover.

repeater radio An additional GNSS radio that is configured as a repeater to be used to achieve lengthy baselines during RT positioning.

Root Mean Square (RMS) Mathematically, it is the square root of the average of the sum of the squared residuals from the computed value. Regarding the solution, it is a measure of predictive power depicted as a spread of the results. For RT positioning, RMS error is usually expressed as $\mathrm{x}, \mathrm{y}$, and $\mathrm{z}$ (up), at the 68 percent (or 1 sigma) confidence. These values should be doubled to express at the 95-percent (or 2 sigma) confidence level.

rover A mobile GNSS receiver that receives corrections from a stationary base or reference station. Typically used in real-time (RT) positioning to establish objective points.

\section{S}

Signal to Noise Ratio (SNR) The ratio of incoming signal strength to the amount of interfering noise as measured in decibels on a logarithmic scale. GNSS measurements have sufficient reliability if the SNR is 30 or greater.

survey-grade GPS/GNSS High precision (centimeter level) positioning using dualfrequency receivers containing both L-Band signals $\mathrm{L}_{1}$ and $\mathrm{L}_{2}$.

\section{T}

trilateration A method of determining a relative terrestrial position using the geometry of three-dimensional spheres from satellite locations and the terrestrial location. This mathematical principal makes the calculation knowing the location of at least three satellites above the terrestrial location and the distance between the terrestrial location and the satellites.

troposphere The troposphere is an expanse of the atmosphere, extending approximately 30 miles from the Earth's surface, that is filled with water vapor and produces our local weather. The wet component of the troposphere is the most difficult to model regarding Global Navigation Satellite System (GNSS) signal delays. Modeling within the troposphere estimates delays as a function of satellite elevation angle, receiver height, and weather components, such as temperature, pressure, and humidity.

\section{U}

UTC (Coordinated Universal Time) The atomic time standard basis of our everyday time keeping. Using highly precise atomic clocks, time is kept in many locations around the world, including the U.S. Naval Observatory. UTC is readily obtained from GNSS satellites.

\section{V}

W

World Geodetic System 1984 (WGS 84) A global geodetic datum defined and maintained by the Department of Defense. Control segments and broadcast ephemeris are expressed in this datum; as a result, Global Navigation Satellite System (GNSS) positioning results are in this datum. WGS 84 positions differ from NAD 83 by between 1 and 2 meters.

\section{$\mathbf{X}$}

XML Known as EXtensible Markup Language, and used to structure, store, and transport information. Software must facilitate sending, receiving, or displaying information.

Y 
Appendixes 1-4 


\section{Appendix 1. Benchmark Recovery and Establishment Form}

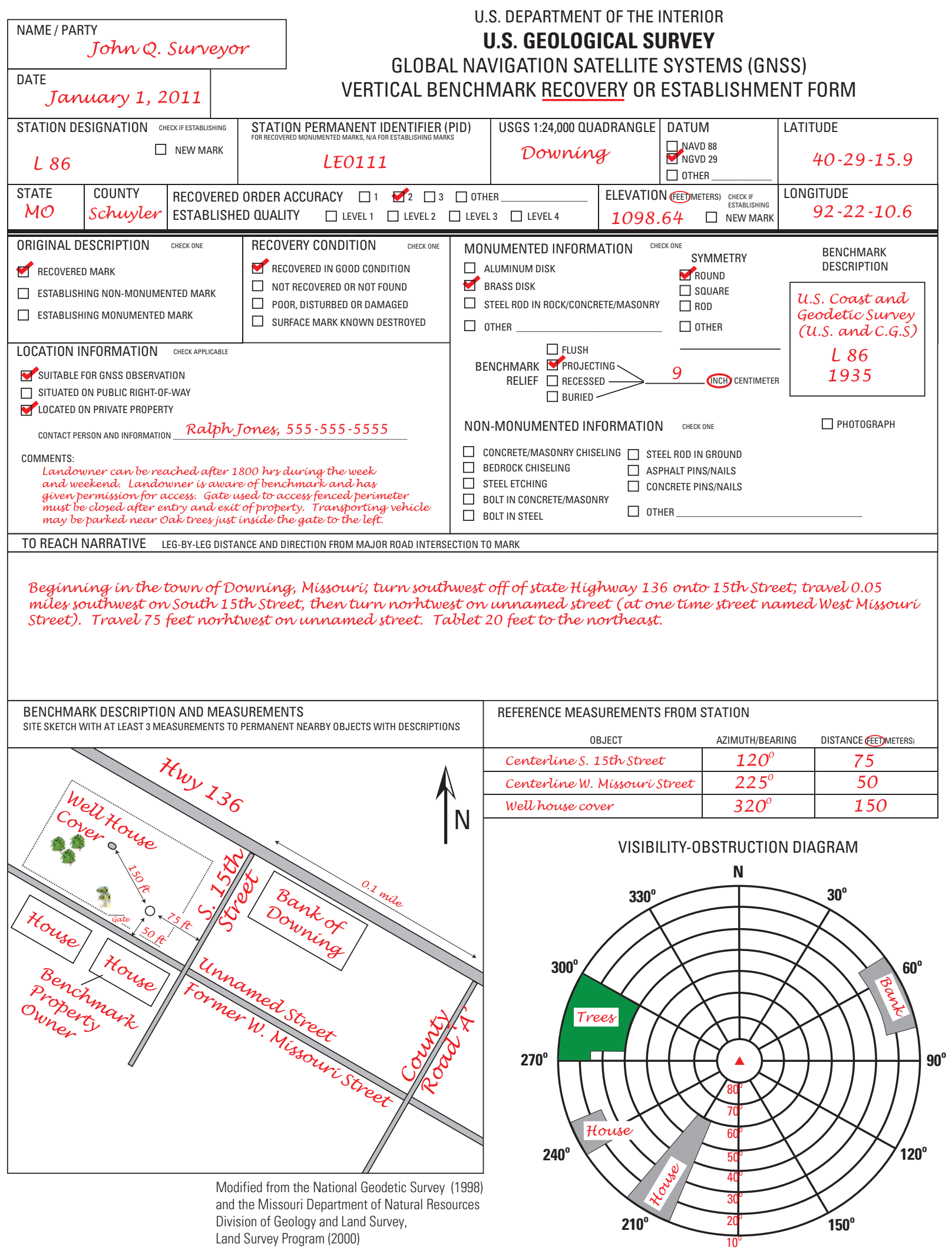




\begin{tabular}{|c|c|c|c|c|c|c|c|c|c|}
\hline \multicolumn{4}{|c|}{$\begin{array}{l}\text { NAME / PARTY } \\
\text { John Q. Surveyor }\end{array}$} & \multirow{2}{*}{\multicolumn{6}{|c|}{$\begin{array}{c}\text { U.S. DEPARTMENT OF THE INTERIOR } \\
\text { U.S. GEOLOGICAL SURVEY } \\
\text { GLOBAL NAVIGATION SATELLITE SYSTEMS (GNSS) } \\
\text { VERTICAL BENCHMARK RECOVERY OR ESTABLISHMENT FORM }\end{array}$}} \\
\hline \multicolumn{4}{|c|}{ DATE January 1, 2011} & & & & & & \\
\hline \multicolumn{3}{|c|}{$\begin{array}{c}\text { STATION DESIGNATION CHECK F ESTABLSHING } \\
\text { OC1 }\end{array}$} & \multicolumn{3}{|c|}{$\begin{array}{l}\text { STATION PERMANENT IDENTIFIER (PID) } \\
\text { FOR RECOVERED MONUMENTEO MARKS, NA F FO ESTABUSHHING MARKS } \\
\qquad N / A\end{array}$} & \multicolumn{2}{|c|}{$\begin{array}{l}\text { USGS 1:24,000 OUADRANGLE } \\
\text { Downing }\end{array}$} & $\begin{array}{l}\text { DATUM } \\
\text { Q NAVD } 88 \\
\text { NGVD } 29 \\
\square \text { OTHER } \\
\end{array}$ & \multirow{2}{*}{\begin{tabular}{|l} 
LATITUDE \\
$\quad 40-29-16.7$ \\
$\begin{array}{r}\text { LONGITUDE } \\
92-22-09.5\end{array}$
\end{tabular}} \\
\hline $\begin{array}{r}\text { STATE } \\
\text { MO }\end{array}$ & $\begin{array}{l}\text { COUNTY } \\
\text { Schuyler }\end{array}$ & \multicolumn{2}{|c|}{$\begin{array}{l}\text { RECOVERED ORDER AC } \\
\text { ESTABLISHED QUALITY }\end{array}$} & $\begin{array}{l}\text { RACY } \square 1 \quad \square 2 \quad \square 3 \\
\square \text { LEVEL } 1 \quad \square \text { LEVEL 2 }\end{array}$ & & & \multicolumn{2}{|c|}{ 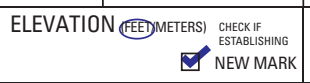 } & \\
\hline \multicolumn{3}{|c|}{$\begin{array}{l}\text { ORIGINAL DESCRIPTION CHEGK ONE } \\
\square \text { RECOVERED MARK } \\
\square \text { ESTABLISHING NON-MONUMENTED MARK } \\
\square \text { ESTABLISHING MONUMENTED MARK }\end{array}$} & \multicolumn{2}{|c|}{$\begin{array}{l}\text { RECOVERY CONDITION CHECK ONE } \\
\square \text { RECOVERED IN GOOD CONDITION } \\
\square \text { NOT RECOVERED OR NOT FOUND } \\
\square \text { POOR, DISTURBED OR DAMAGED } \\
\square \text { SURFACE MARK KNOWN DESTROYED }\end{array}$} & \multirow{2}{*}{\multicolumn{4}{|c|}{ 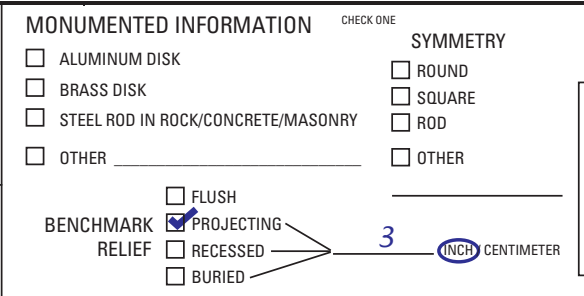 }} & Centerpunched \\
\hline \multirow{3}{*}{\multicolumn{5}{|c|}{ 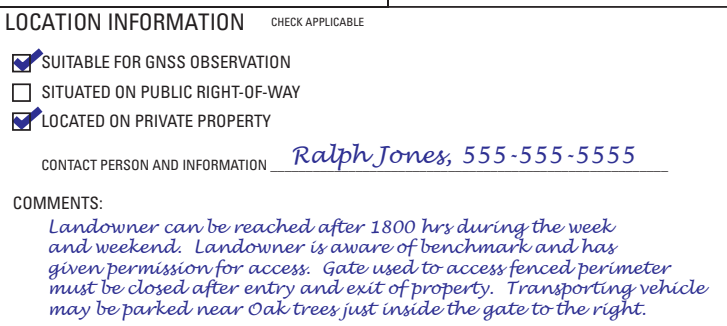 }} & & & & & \\
\hline & & & & & \multirow{2}{*}{\multicolumn{4}{|c|}{ 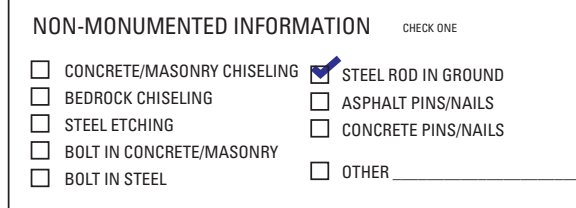 }} & $\square$ PHOTOGRAPH \\
\hline & & & & & & & & & \\
\hline
\end{tabular}

TO REACH NARRATIVE LEG-BY-LEG DISTANCE AND DIRECTION FROM MAJOR ROAD INTERSECTION TO MARK

Beginning in the town of Downing, Missouri, turn southwest off of state Highway 136 onto 15 th Street, travel 0.05 miles southwest on South 15 th Street, then turn norhtwest on unnamed street (at one time street named West Missouri Street). Travel 75 feet norhtwest on unnamed street. Rebar 170 feet to the northeast.

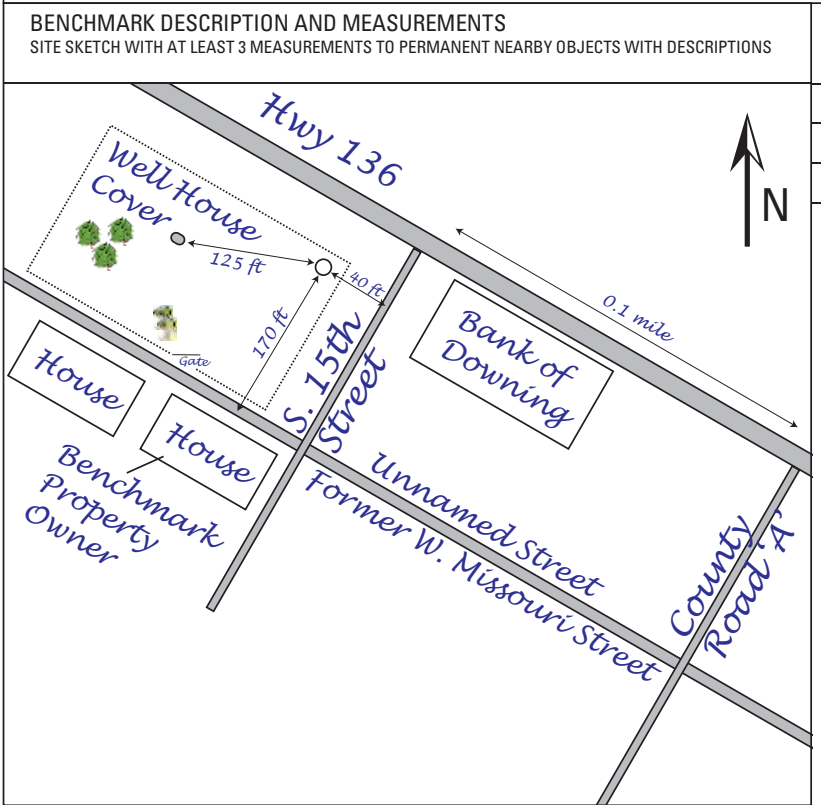

Modified from the National Geodetic Survey (1998 and the Missouri Department of Natural Resources Division of Geology and Land Survey, Land Survey Program (2000)
REFERENCE MEASUREMENTS FROM STATION

\begin{tabular}{|l|c|c|}
\multicolumn{1}{c|}{ OBJECT } & AZIMUTH/BEARING & DISTANCE EEETMETERS \\
\hline Centerline S. 15th Street & $120^{\circ}$ & 40 \\
\hline Centerline W. Missouristreet & $225^{\circ}$ & 170 \\
\hline Well house cover & $285^{\circ}$ & 125 \\
\hline
\end{tabular}

VISIBILITY-OBSTRUCTION DIAGRAM

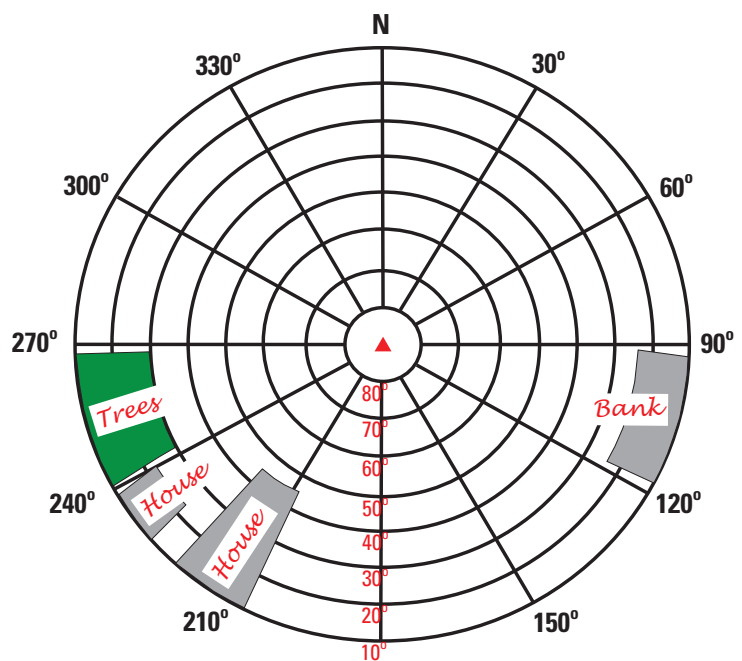




\title{
Appendix 2. Static Observation Form
}

\author{
U.S. DEPARTMENT OF THE INTERIOR \\ U.S. GEOLOGICAL SURVEY \\ GLOBAL NAVIGATION SATELLITE SYSTEMS (GNSS) \\ STATIC (BASE STATION) OBSERVATION FORM
}

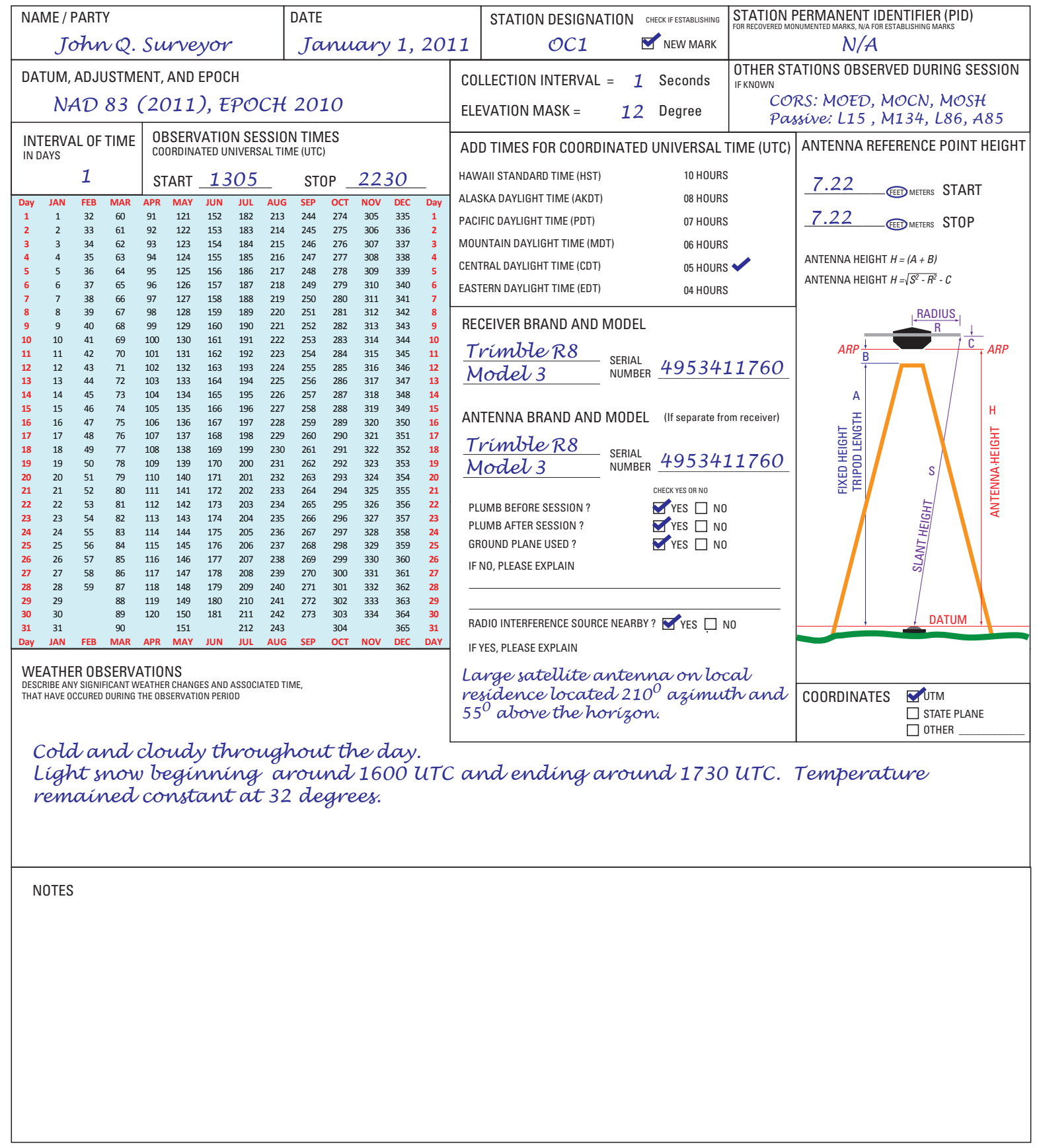

Modified from the National Geodetic Survey (1998) and the Missouri Department of Natural Resources Division of Geology and Land Survey,

Land Survey Program (2000) 


\section{Appendix 3. Real-Time GNSS Example Approaches}

\section{High-Water Mark Surveys-Flood Studies}

High-water mark surveys encountered most often using GNSS are those performed for flood-related studies to determine inundation extents. These studies combine surveyed high-water marks in the field along with various topographic details, such as artificial and natural control features, river channels, roadways, floodplains, and any other detail necessary to hydraulically model flood flows. As an example, a flood study is performed along a 16-km reach of the Salt River near Brashear, Missouri (fig. 3-1).

There are two trusted and recovered second-order benchmarks at Hurdland and Brashear (fig. 3-1). There is one third-order benchmark at Gibbs (fig. 3-1). Mission planning regarding space weather, PDOP, and satellite availability dictate general avoidance times of 1100-1130 and 1530-1730 during the week of the scheduled campaign. A RTN is available in this example and the objective of the flood study leads the survey chief to engage in a Level II campaign. The campaign documents metadata, as described earlier in the "Metadata" section of this manual, for a RTN in addition to utilizing recovery and establishment forms (appendix 1). As previously described, the following assurances are maintained within the campaign:

- Benchmark evaluation, reconnaissance, field preparation, and data storage.

- Bubble check and calibration of base fixed-height tripod and rover bipod.

- Base fixed-height or dual-clamped tripod stabilized by chains or sandbags.

- Rover multipath avoidance for each observation session.

- Vertical precision less than $0.05 \mathrm{~m}$ (2 sigma) during observations.

- RT blunder checks within $0.03 \mathrm{~m}$.

- 180 epochs of data per observation (1-second collection interval for 3 minutes).

- PDOP assurances of three or less.

- Satellite availability of seven or more.

- Second-order benchmarks used to localize or check within $0.05 \mathrm{~m}$.

The survey chief ensures that the RTN is aligned to the NSRS and discovers it is NAD 83 (CORS96) 2002.0.
Procedurally for a Level II approach, a localization may begin at the second-order benchmark at Brashear. The user then observes the second-order mark at Hurdland and thirdorder mark at Gibbs. Vertical residuals produced from a least-squares fit of these known benchmarks to observations performed at each benchmark produce the following results:

- Brashear $0.006 \mathrm{~m}$

- Hurdland $0.010 \mathrm{~m}$

- Gibbs $0.005 \mathrm{~m}$

In this case, Brashear and Gibbs have sufficient agreement, but are second- and third-order marks. A Level II campaign requires second-order benchmarks to be localized or checked, and Hurdland is only $0.004-0.005 \mathrm{~m}$ high from Brashear and Gibbs, so the user is reluctant to identify Hurdland as an outlier and omit. The user decides to localize to Brashear and Gibbs, and commences observations on all objective points. The use of a differential level or total station instrument will be used to perpetuate elevation from these objective points to the high-water mark. Using a Level II approach, all observations are performed using RT blunder checks. Each redundant shot is ensured to be within $0.03 \mathrm{~m}$, and those that are not are held suspect and re-established at a different location to achieve that uncertainty. The user keeps sufficient record of all field procedure (as described previously under "GNSS Campaign"), including PDOP, satellite availability, rover rod heights, and all redundancy differences. After all objective points are established; the user re-occupies two objective points (approximately 10 percent of 19) and is within $0.05 \mathrm{~m}$ of each, then occupies Hurdland and checks within $0.05 \mathrm{~m}$. Finally, the user re-occupies Brashear as a "closing" check and ensures the observation is within $0.05 \mathrm{~m}$ of the stated benchmark elevation.

In this example, if the user had checked on Hurdland and exceeded $0.05 \mathrm{~m}$, it is at the discretion of the survey chief to evaluate the entire campaign for an appropriate classification. If Hurdland checked at $0.08 \mathrm{~m}$ (greater than $0.05 \mathrm{~m}$ ), yet all objective point redundancy was within $0.03 \mathrm{~m}$, and all other criteria related to satellite availability, PDOP, observation length, and multipath avoidance were maintained, then this campaign would be inclined to sustain a Level II classification. Conversely, if many observations were performed with unfavorable PDOP, many redundant residuals were not within $0.03 \mathrm{~m}$, there was no sufficient documentation to demonstrate potential multipath conditions, and there were occurrences of communication disruption because of power issues (including delays acquiring a fixed solution), the classification would likely be downgraded to a Level III campaign. 


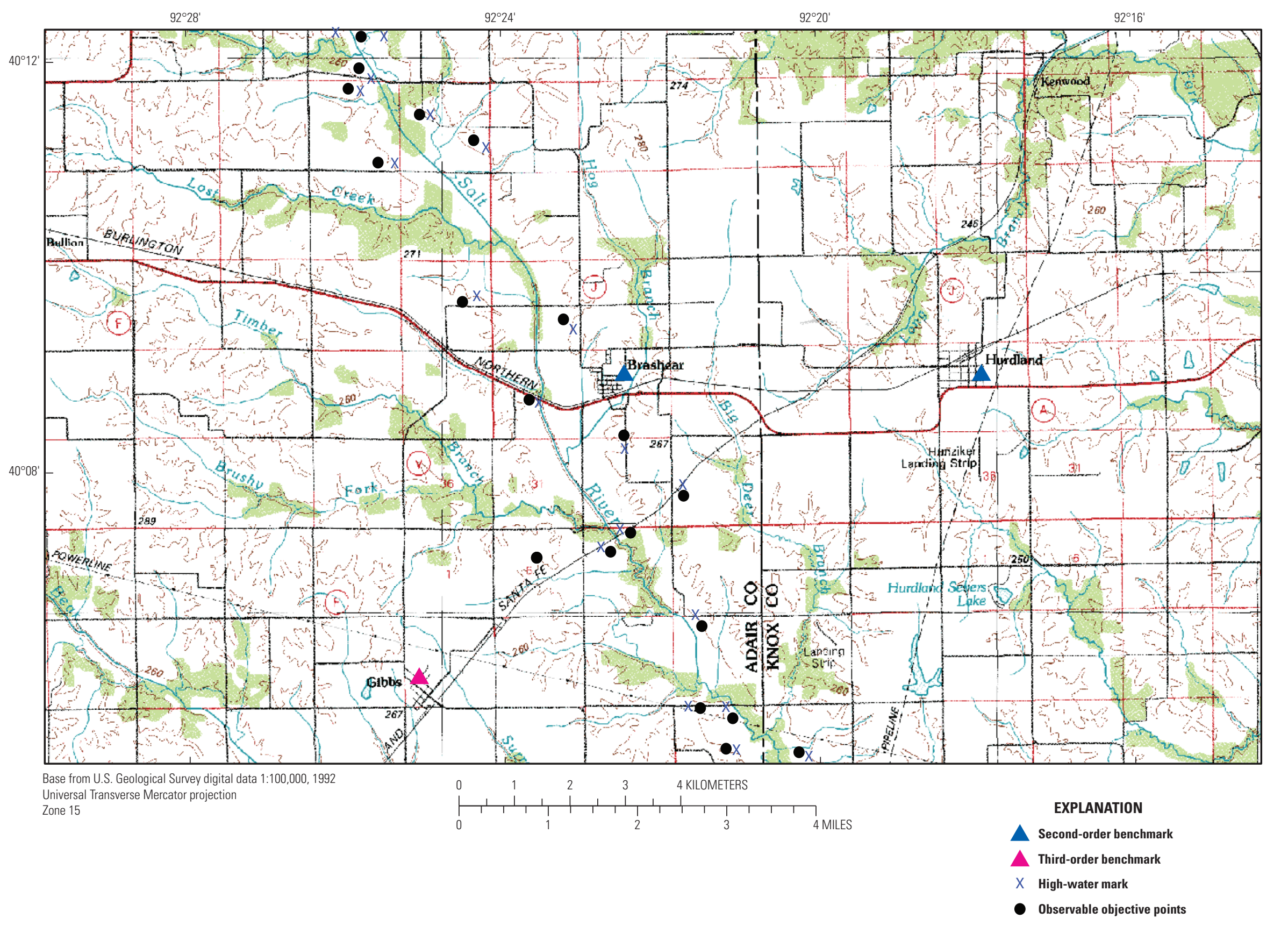

Figure 3-1. Background for a high-water mark survey along the Salt River near Brashear, Missouri. 


\section{Establishment of Datum at Streamgages}

Using a method to establish vertical datum at a USGS streamgage often requires an evaluation of the gaging effort and importance to the cooperative water community. The quality of approach used in establishing datum for a gage with forecasting, commerce, and regulation interests may be different than an approach used to establish datum at a gage with only recreational interests. Another decision made in this regard may include the amount of relief in the watershed, and resulting gradient and surge inherent in the gaged water surface.

Referring to figure 3-2, 14 streamgages need to be tied to datum in a 2,100 square mile $\left(\mathrm{mi}^{2}\right)$ area. These gages are operated and maintained in cooperation with the U.S. Army Corps of Engineers (USACE), and are used to support Mississippi River navigation and forecasting. Based on cooperative interest, navigation, and forecasting driving the overall objective, a Level II approach will satisfy the required quality. A RTN is available in this example and will be used to perpetuate datum near or at each streamgage. In this example, 12 second-order benchmarks and 1 third-order benchmark were recovered; however, only 7 second-order benchmarks and 1 third-order benchmark were observable and accepted for this GNSS campaign. Mission planning regarding space weather, PDOP, and satellite availability dictate general avoidance times of 1000-1030 and 1730-1830 during the week of this scheduled campaign. The campaign documents metadata, as described earlier, for a RTN in addition to utilizing recovery and establishment forms (appendix 1). As described in the previous example, the following assurances are maintained within this campaign:

- Benchmark evaluation, reconnaissance, field preparation, and data storage.

- Bubble check and calibration of base fixed-height tripod and rover bipod.

- Base fixed-height or dual-clamped tripod stabilized by chains or sandbags.

- Rover multipath avoidance for each observation session.

- RT blunder check within $0.03 \mathrm{~m}$.

- Vertical precision less than $0.05 \mathrm{~m}$ (at 2 sigma) during observations.

- 180 epochs of data per observation (1-second collection interval for 3 minutes).

- PDOP assurances of three or less.

- Satellite availability of seven or more.

- Second-order benchmarks used to localize or check within $0.05 \mathrm{~m}$.
The survey chief ensures that the RTN is aligned to NSRS and discovers it is NAD 83 (NSRS2007) 2007.0. The GNSS user begins conducting observations at all second- and third-order benchmarks in the field (fig. 3-2). The GNSS user notes intermittent cellular communication for marks 7 , 8 , and 9 in the field and employs a RTK bridge at a cellular receptive location that is elevated and centralized to marks 7, 8, and 9 (fig. 3-2). A check (in lieu of a localization or site calibration) of known elevations opposed to observed elevations provided in table 3-1 indicates use of benchmarks 2-7, 10 , and 13 because of the spatiality and confidence of these benchmarks as well as consistency among residuals.

Using a Level II approach, all objective points are established using a RT blunder check. As with the previous example, each redundant shot is ensured to be within $0.03 \mathrm{~m}$, and those that are not are held suspect and re-established at a different location to achieve that uncertainty. The user keeps a sufficient record of all field procedures as described previously in the "GNSS Campaign" section. After all objective points are established, the user re-occupies two objective points (approximately 10 percent of 14) along with benchmarks 2-7, 10 , and 13 , and is within $0.05 \mathrm{~m}$ of the initial observation of the objective point and $0.05 \mathrm{~m}$ from the benchmark elevation.

Similar to the previous example, if the user had exceeded $0.05 \mathrm{~m}$ when checking on benchmarks at the end of the campaign, it is at the discretion of the survey chief to evaluate the entire campaign for an appropriate classification. A record of satellite availability, PDOP, observation length, multipath avoidance, communication issues, and suspect delays acquiring a fixed solution are evaluated to sustain or downgrade the level quality. In this example, USACE and the National Weather Service (NWS) have inquiries regarding a numerical representation of vertical datum accuracy. The survey chief provides to both interests an executive summary released through an appropriate USGS information product (http:// www.usgs.gov/usgs-manual/1100/1100-3.html) which includes the following:

- Level II quality requirements for a RT approach. If this campaign was downgraded to a Level III effort, discussion is needed to justify.

- Metadata.

- A table that includes:

- Residuals from comparing known to observed benchmarks for perpetuating datum (table 3-1).

- Residuals from objective point establishment using an RT blunder check.

- End of campaign; residuals from comparing known to observed benchmarks for benchmarks 2-7, 10, and 13 .

- Residuals from 10 percent of re-occupied objective points. 

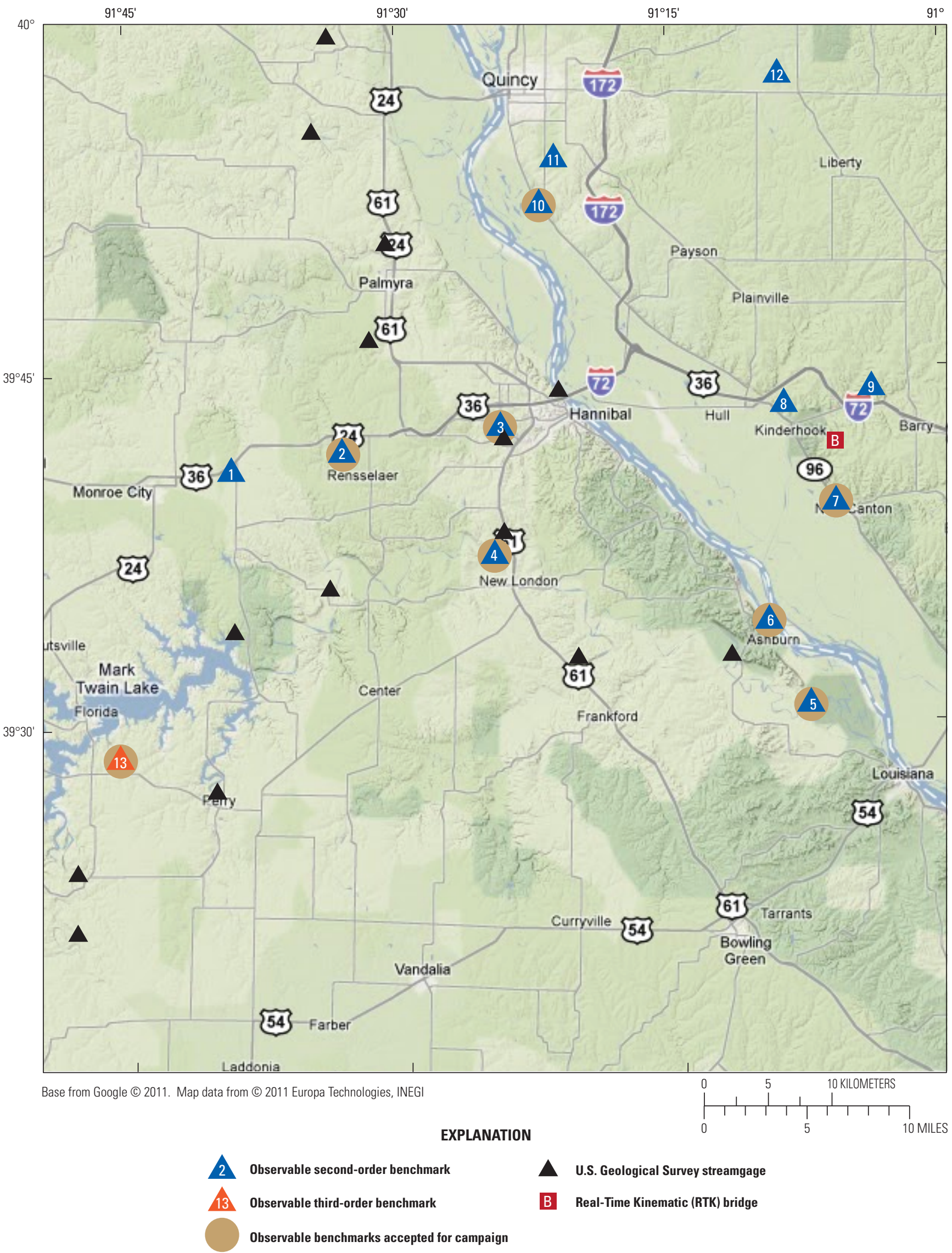

U U.S. Geological Survey streamgage

B Real-Time Kinematic (RTK) bridge

Figure 3-2. Background for streamgage datum establishment. 
- An overall uncertainty for the campaign and discussion regarding the benchmarks held as "truth."

Table 3-1. Assessment of benchmarks for perpetuating datum to U.S. Geological Survey streamgages.

$[\mathrm{m}$, meter $]$

\begin{tabular}{cccccc}
\hline Benchmark & Order & $\begin{array}{c}\text { Known } \\
\text { elevation } \\
(\mathbf{m})\end{array}$ & $\begin{array}{c}\text { Observed } \\
\text { elevation } \\
(\mathbf{m})\end{array}$ & $\begin{array}{c}\text { Residual } \\
(\mathbf{m})\end{array}$ & Accept \\
\hline 1 & 2 & 224.017 & 223.935 & 0.082 & No \\
2 & 2 & 229.807 & 229.745 & 0.042 & Yes \\
3 & 2 & 232.987 & 232.937 & 0.040 & Yes \\
4 & 2 & 182.871 & 182.923 & -0.042 & Yes \\
5 & 2 & 152.392 & 152.433 & -0.031 & Yes \\
6 & 2 & 155.462 & 155.505 & -0.033 & Yes \\
7 & 2 & 143.639 & 143.586 & 0.043 & Yes \\
8 & 2 & 181.234 & 181.169 & 0.065 & No \\
9 & 2 & 182.369 & 182.219 & 0.150 & No \\
10 & 2 & 190.491 & 190.496 & -0.005 & Yes \\
11 & 2 & 189.271 & 189.128 & 0.143 & No \\
12 & 2 & 234.124 & 234.211 & -0.087 & No \\
13 & 3 & 219.445 & 219.403 & 0.032 & Yes \\
\hline & & & & &
\end{tabular}

\section{Establishment of Datum at Groundwater Well Fields}

Before establishing a datum for groundwater wells, the project staff should refer to Cunningham and Schalk (2012) for the procedures to establish permanent measurement points and other reference marks for wells and documenting the location of wells. For the surveyor and project staff, it is important to discuss what the accuracy requirements are for the project, and communicate the expected accuracy, relative to the cost of the GNSS survey (time per station). For some groundwater projects, the hydraulic gradient of a groundwater well field generally dictates level quality concerns regarding establishment of datum. The well field may be monitoring a potentiometric surface with substantial relief, or a well field may reside in an alluvial plain with minimal relief. For either circumstance, it is important to recognize the limitations of GNSS surveys when relating altitude among groundwater wells and to communicate with the project staff about these limitations. For smaller alluvial well fields that do not exhibit relief, it is generally acceptable to establish a benchmark that is central to the well field and perpetuate this altitude to each groundwater well by differential leveling. For larger well fields that monitor a larger gradient change, it may be acceptable to select a GNSS approach to provide an independent geodetic tie to each groundwater well.

For example, a groundwater monitoring well field resides within a $50-\mathrm{mi}^{2}$ area (fig. 3-3).
Altitude of the potentiometric surface needs to be expressed to the nearest $0.5 \mathrm{ft}(0.152 \mathrm{~m})$. A RTN does not exist in this example and the survey chief has concluded from the use of the data that a Level III approach using singlebase RTK will ensure the required quality needs will be met. Mission planning identifies a reduction in available satellites and poor PDOP around 1000 and between 1600-1800 for scheduled days of GNSS observation. No satellite outages or compromising space weather was predicted for the scheduled effort. For the Level III approach, the following assurances are maintained within the campaign:

- Benchmark evaluation, reconnaissance, field preparation, and data storage.

- Bubble check and calibration of base fixed-height tripod and rover bipod.

- Base fixed-height or dual-clamped tripod stabilized by chains or sandbags.

- Rover multipath avoidance for each observation session.

- Recovery, establishment, and observation forms used to document existing or established benchmarks (appendixes 1 and 2).

- Height of base Antenna Reference Point (ARP) measured before and after observation session.

- RT blunder check within $0.03 \mathrm{~m}$.

- Vertical precision less than $0.07 \mathrm{~m}$ (at 2 sigma) during observations.

- 180 epochs of data collected per observation (1 second collection interval for 3 minutes).

- Baseline lengths may not exceed $9 \mathrm{~km}$.

- PDOP may be less than or equal to four.

- Satellites observed may be greater than or equal to six.

- Localizations or checks may occur for vertical order 3 or better benchmarks or a Level II OPUS-S substitute may be made for any benchmark (within $0.05 \mathrm{~m}$ ).

Initial reconnaissance combined with terrain mapping identifies three second-order vertical control benchmarks that are observable (fig. 3-3). Similar to the Level II approach, a Level III approach requires two benchmarks in which to localize and check for each base station occupation. In this case, single-base RTK is "used as level." All base station locations are autonomous, which provides a reduction in baseline length and added flexibility in localizing and checking benchmarks, and establishing objective points. In this campaign, extended range poles equipped with whip antennas are used at the base and rover receivers for radio communication assurance. 


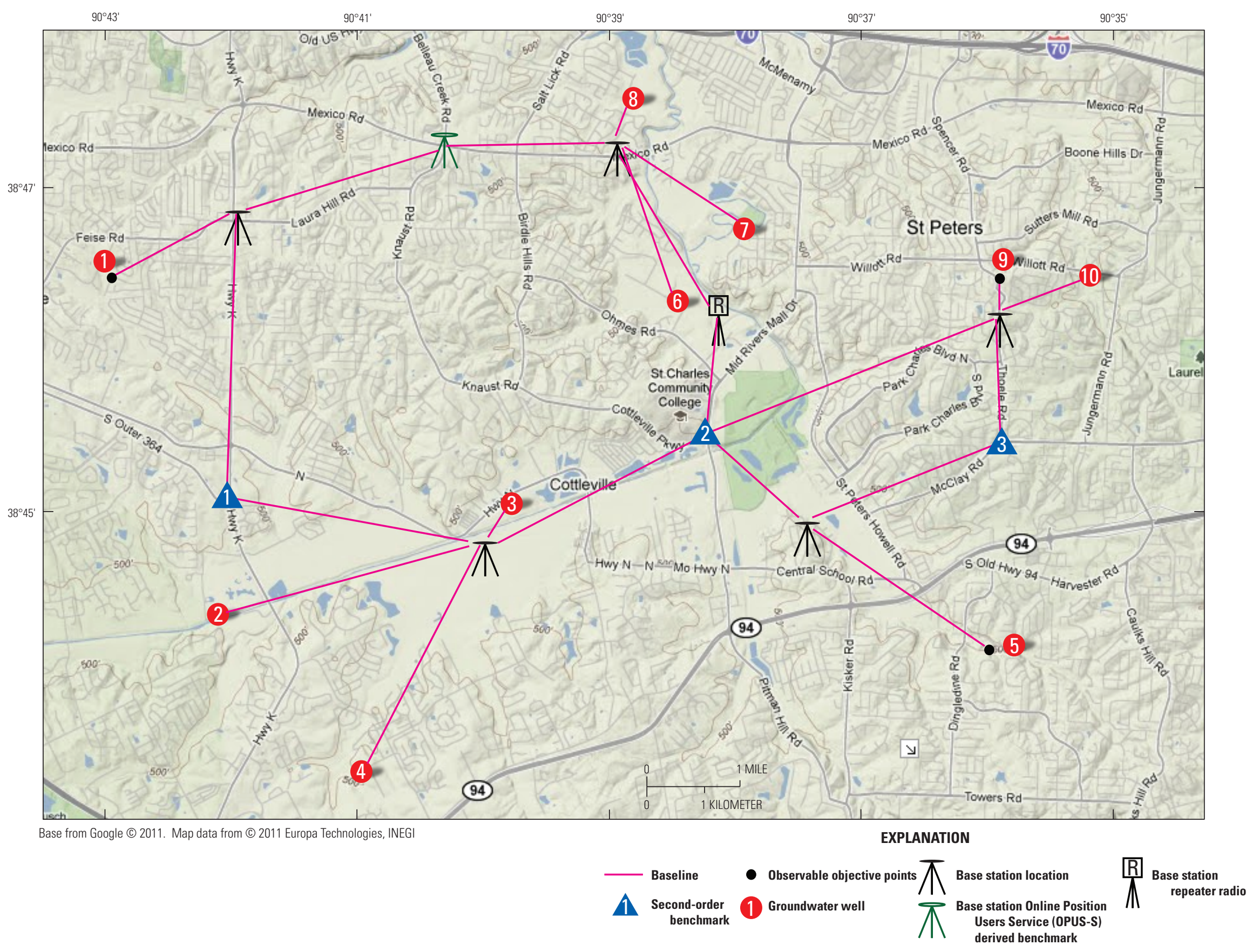

Figure 3-3. Background for a groundwater well field survey. 
Reconnaissance and terrain mapping identified length and direction of potential baselines based on areas of relief indicating predictable radio communication.

With the exception of well locations 1,5 , and 9 , a reference mark at all other well locations were suitable for direct observations (fig. 3-3). For groundwater well locations 1, 5, and 9, encroaching tree canopy and transmission lines compromised a quality observation. As a result, observable objective points were established near the groundwater well so that differential leveling could be used to perpetuate altitude. Six base station occupations were required to perform RT observations (fig. 3-3). An additional repeater radio was required when conducting observations for wells 6,7 , and 8 . This repeater radio was used to facilitate a localization to benchmark 2. Because there was no second benchmark to check within the vicinity of wells 6,7 , and 8 , a Level II quality static OPUS-S solution was derived to provide this check (fig. 3-3). All redundant observations are part of a RT blunder check within the abbreviated chronology of campaign observations listed below:

1. Level II single-base static (OPUS-S) occupation and data collection begins between wells 1 and 8 . Concurrently, RT campaign commences.

2. Base station occupation between benchmark 2 and well 2, which includes localization to benchmark 2; the establishment of objective points using a RT blunder check at wells 2,3 , and 4 ; a check on benchmark 1; and a closing check back on benchmark 2 .
3. Base station occupation between benchmark 2 and well 5 , which includes a localization to benchmark 2; the establishment of an objective point using a RT blunder check at well 5; a check on benchmark 3; and a closing check back on benchmark 2 .

4. Base station occupation between benchmark 3 and well 9, which includes a localization to benchmark 3; the establishment of objective points using a RT blunder check at well 9 and 10; a check on benchmark 2; and a closing check back on benchmark 3 .

5. Process OPUS-S collection that occurred between wells 1 and 8 .

6. Base station occupation between well 1 and an OPUS-S derived benchmark, which includes a localization to benchmark 1; the establishment of an objective point using a RT blunder check at well 1; a check on the OPUS-S derived benchmark, and a closing check back on benchmark 1 .

7. Base station occupation between well 7 and an OPUS-S derived benchmark, which includes a localization by way of repeater radio to benchmark 2; the establishment of objective points using a RT blunder check at wells 6, 7, and 8; a check on the OPUS-S derived benchmark; and a closing check back on benchmark 2 . 


\section{Appendix 4. Static GNSS Example Approaches}

\section{Single-Base Static (OPUS)—Datum Establishment for River Survey}

Bathymetric and topographic surveys are planned along eight U.S. Army Corps of Engineers historic sediment transects for Lewis and Clark Lake, which is located on the Missouri River between Nebraska and South Dakota. A Level IV survey is all that is required for the topographic part of the survey, but a Level III survey is needed to establish objective points and water surface elevations necessary to process bathymetry data. A RTN is not available; therefore, all RT surveys will be performed with single-base RTK.

NGS benchmarks with second-order vertical accuracy, or better, elevation were recovered from the NGS IDB. These are grouped into two sets of marks: those observed in the past using GPS, and those with no prior observations. As figure 4-1 illustrates, many second-order vertical benchmarks are recovered, but most of them were several miles from the reservoir and only three had been previously observed using GPS. Also, only one GPS-observed mark (recovered in 1995) is located on the southwest side of the reservoir. The two other GPS-observed benchmarks near the northeast side of the reservoir had been recovered since 1993. The second-order vertical benchmarks that were close to the edge of the water (and some that were within the reservoir) had not been recovered since the 1940's, before the completion of the dam. Considering the fact that the benchmarks likely to be recovered and observed using GPS were a distance from the reservoir, new benchmarks were established closer to the reservoir for convenience. NGS benchmarks that are located and observed near newly established benchmarks will be used as quality checks in the campaign.

The location of objective points for each new set of benchmarks (two controlling benchmarks required for a Level III RT survey) is selected based on proximity to boat ramps and roads, topography, vegetation, permission, and location in relation to the survey transects (fig. 4-1). Once the location of the "to-be-established" benchmarks is determined, a decision regarding the type and construction of benchmark is made. As described in the manual, there are several options for benchmark construction. Planned locations did not represent a rock-dominated substrate; therefore the use of FENO markers is appropriate. The newly established benchmarks are labeled as follows: benchmarks on the northwest side of the lake are named 1053 and 1054, benchmarks on the southwest side of the lake are named 1052 and 1059, and benchmarks in the middle of the reservoir on the north side are named 1070 and 1071 (fig. 4-1).

After determining where and how benchmarks will be constructed, appropriate stamping for those that are monumented, how elevation will be established at each benchmark, and when the surveying will occur must be decided. A Level II single-base static (OPUS) approach is used to determine elevation at each benchmark. Benchmarks created from this approach satisfy the requirement required to perpetuate elevation in a Level III RTK survey. Before the campaign, mission planning is performed to evaluate optimal days for observations. Also, nearby CORS data are evaluated through the NGS CORS website to ensure all stations are functioning before the survey. Figure 4-2 illustrates a time series and data availability example for nearby CORS station "SDSF." Data indicate SDSF is stable and has reflected a full data set in the recent past (fig. 4-2), which implies this station should be considered a quality CORS to use as part of the data collection effort. All landowners and land managers are contacted for permission to establish benchmarks and conduct surveys. The following assurances are maintained within the campaign:

- Benchmark evaluation, reconnaissance, field preparation, and data storage.

- Bubble check and calibration of base fixed-height tripod and rover bipod.

- Base fixed-height or dual-clamped tripod stabilized by chains or sandbags.

- Recovery, establishment, and observation forms used to document existing or established benchmarks (appendixes 1 and 2).

The field crew constructed 6 new benchmarks during the same day they tried to locate 3 NGS benchmarks that had previously been surveyed by GPS. One of the NGS benchmarks located on the southwest side of the lake, NGS PID NM1382, Designation Santee Reset, is recovered in good condition and utilized as a quality check during the static surveys by collecting data on the benchmark at the same time GPS observations are performed on newly established benchmarks. Concurrent observations provide a quality check of the Level II single-base static (OPUS) derived position against the published position.

Including the NGS benchmark Santee Reset, the field crew has seven benchmarks to collect static data on. The equipment available includes only 5 fixed-height tripods, so only 5 GPS receivers will be collecting static data at any one time; therefore, the field crew begins by setting up on the benchmarks located on the southwest side of the lake because they are the closest to the point of deployment. The crew observes benchmarks 1052 and 1059 from approximately 0600 to 1200 on day 1 . After setting up receivers at 1052 and 1059 , the crew leaves the receivers unattended because of the remote area and deploys two more receivers on benchmarks 1053 and 1054 and initiates observations from 0700 to 1300 


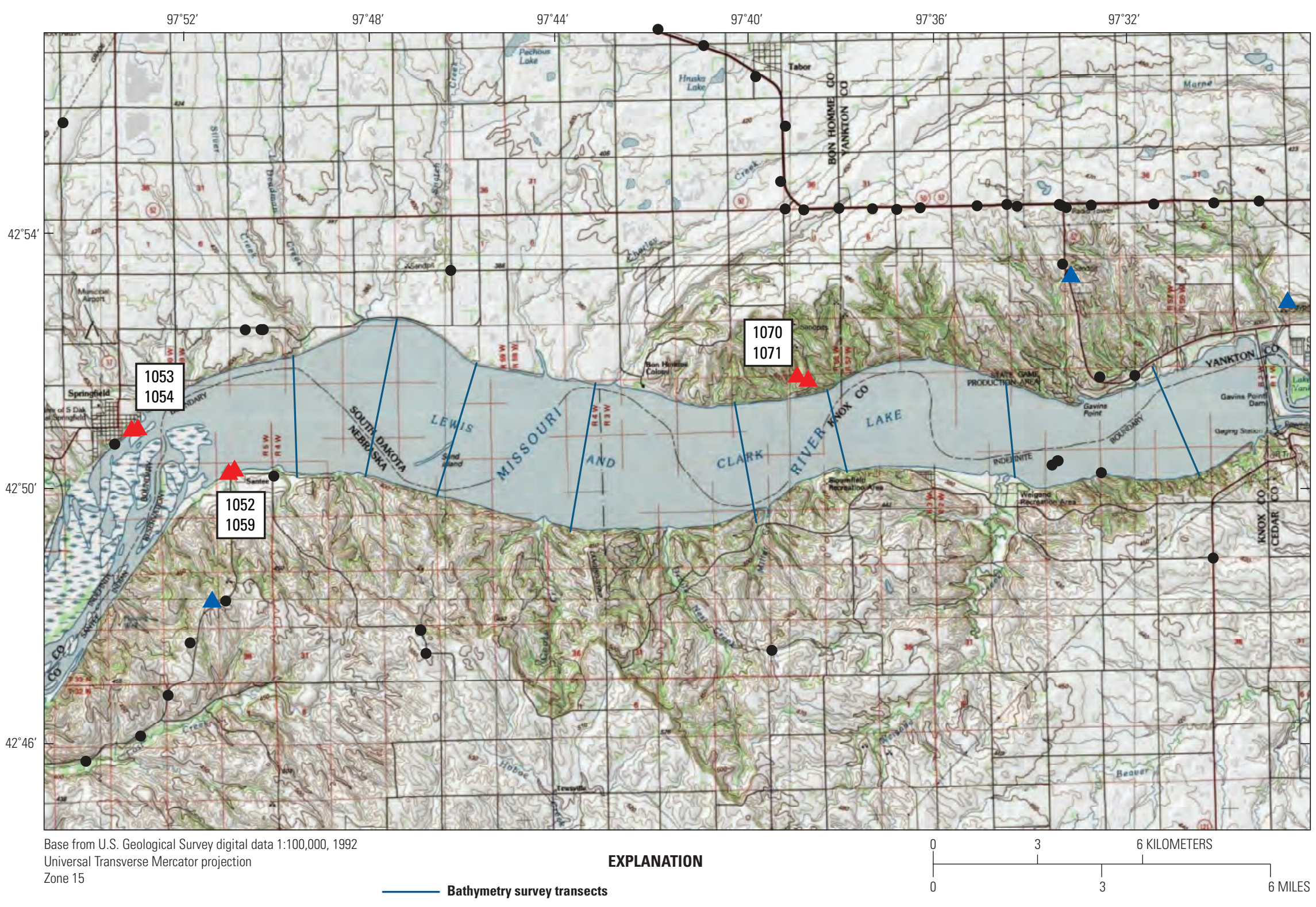

N National Geodetic Survey (NGS) Global Positioning System (GPS) observed benchmarks

1052 Objective points with identifier established using single-base static GPS

- Benchmarks identified in the NGS database (vertical order II)

Figure 4-1. Recovered and established benchmarks for the topographic and bathymetric survey at Lewis and Clark Lake. 


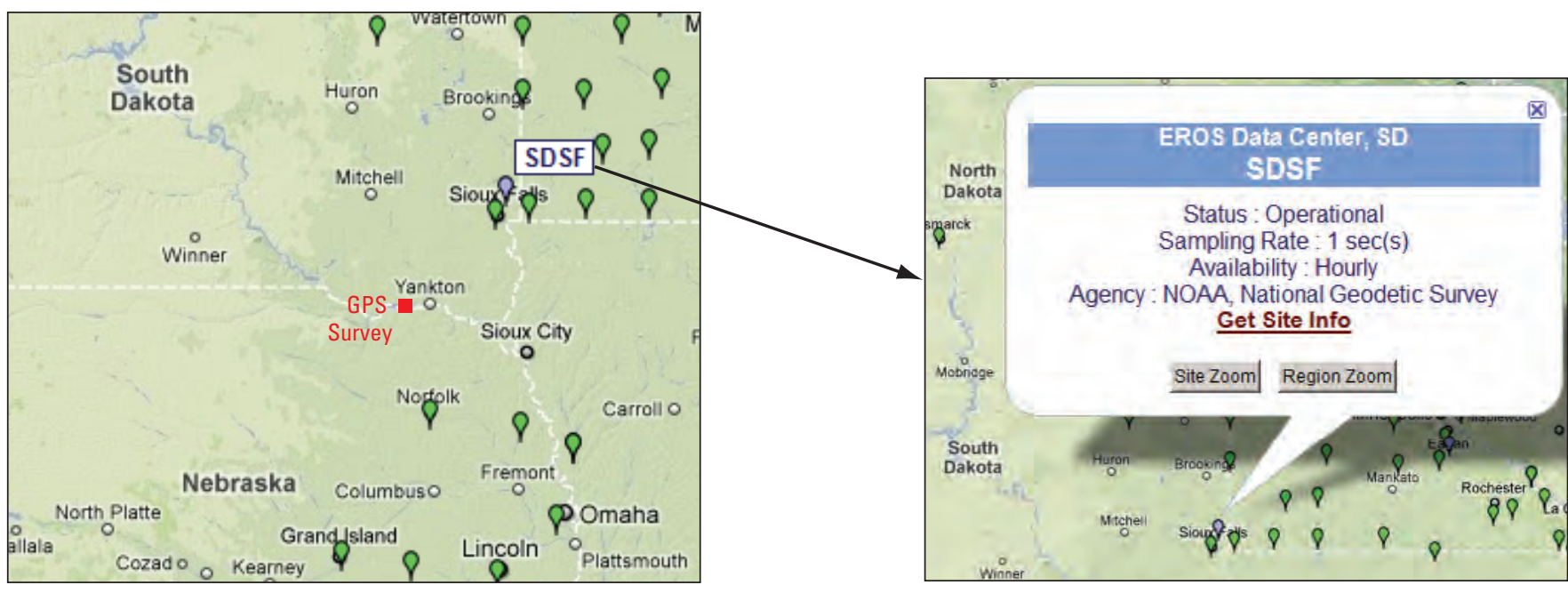

http://www.ngs.noaa.gov/CORS/GoogleMap/CORS.shtml

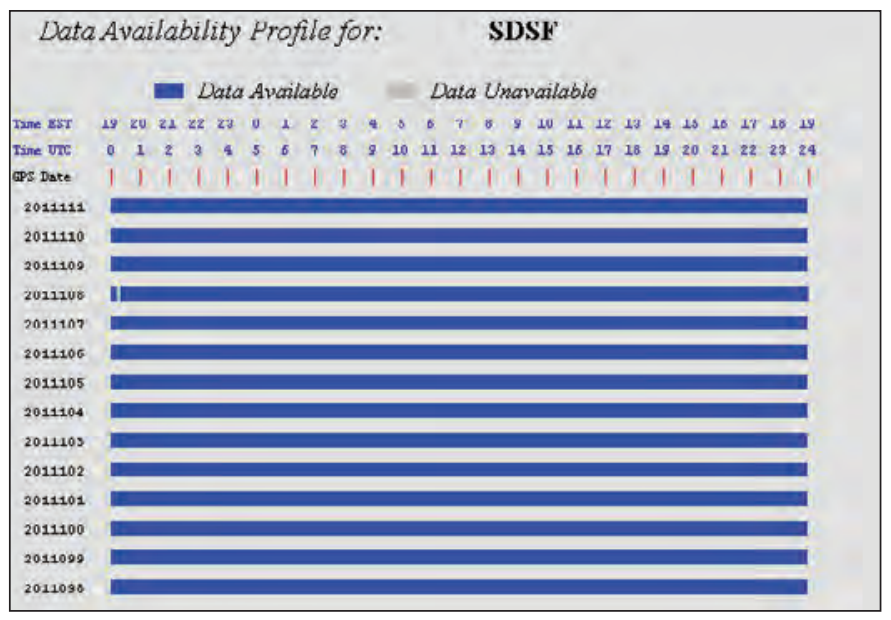

Data available from the station for the last 14 days.

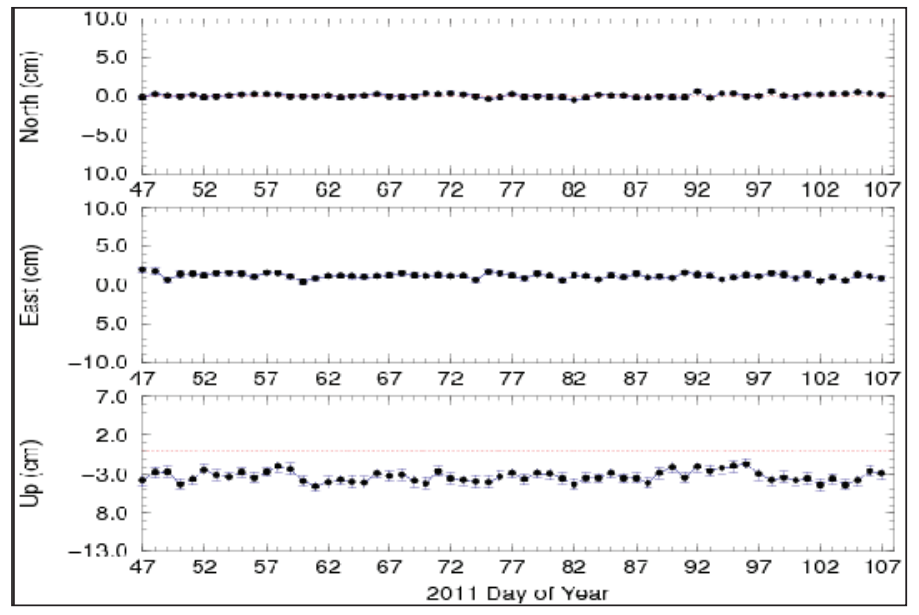

Movement in the northing, easting, and up position for the last 60 days.
http://www.ngs.noaa.gov/CORS/GoogleMap/CORS.shtm/

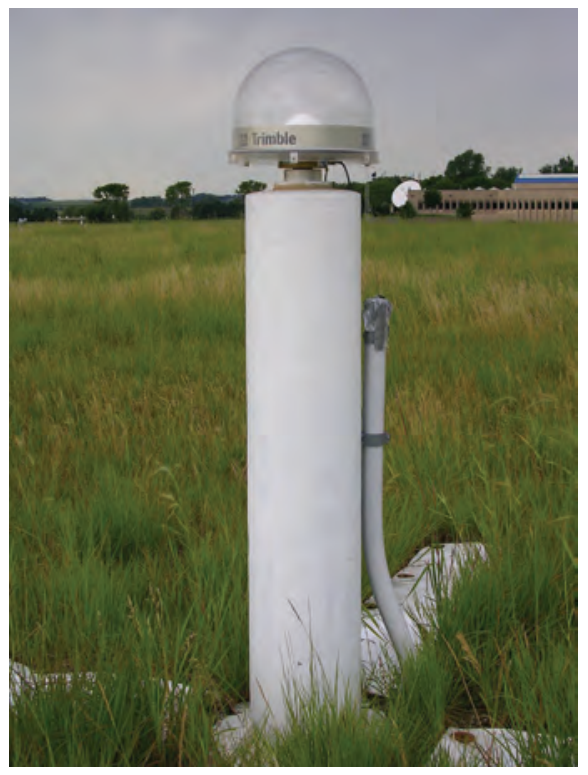

Continuously Operating Reference Station (CORS) SDSF.

Figure 4-2. Time series and data availability plots downloaded from the National Geodetic Survey (NGS) Continuously Operating Reference Station (CORS) website. 
on the same day. After these two receivers are initialized and collecting data, the crew deploys the final tripod and receiver on NGS benchmark Santee Reset, which collects data from 0830 to 1500 . The remaining benchmarks 1070 and 1071, recently constructed for observations, are observed the following day from 0830 to 1430 . These marks are located near the north shore toward the central part of the Lewis and Clark Lake. While observations are being performed on benchmarks 1070 and 1071, the field crew will process 1052, 1053, 1054, and 1059 through OPUS, so that the bathymetry and Level III single-base RTK part of the field work can begin. Processing of these observations will use the ultra-rapid orbit, but a check and potential reprocessing against the rapid or precise orbits will be performed later if the criteria do not meet the requirements for a Level II quality.

Processing is completed by converting observation files to a RINEX format. Formatted observation files, the surveyor's email address, receiver antenna type, and vertical height from the established or recovered benchmark to the ARP are entered into OPUS. For OPUS processing to meet a Level II single-base static survey, a minimum observation time of 4 hours must be ensured with the following processing results:
- Total observations used: 80 percent or more.

- Ambiguities fixed: 80 percent or better.

- Overall RMS: $<0.03$ m.

- Vertical peak-to-peak error: $<0.08 \mathrm{~m}$.

With the exception of time requirements for benchmarks 1052 and 1053, results processed for all newly established benchmarks met these requirements. An example of benchmark 1052 is indicated below and quality indicators are highlighted in red. Quality indicators for the remaining benchmarks are summarized in table 4-1.

Table 4-1. Benchmark quality indicators from Online Position Users Service (OPUS) results for datum establishment used for a river survey.

[hrs, hours; RMS, Root Mean Squared error; m, meter]

\begin{tabular}{cccccrcc}
\hline $\begin{array}{c}\text { Bench- } \\
\text { mark }\end{array}$ & $\begin{array}{c}\text { Time } \\
\text { (hrs) }\end{array}$ & $\begin{array}{c}\text { Observations } \\
\text { used }\end{array}$ & Percent & $\begin{array}{c}\text { Fixed } \\
\text { ambiguities }\end{array}$ & Percent & $\begin{array}{c}\text { Overall } \\
\text { RMS } \\
\text { (m) }\end{array}$ & $\begin{array}{c}\text { Orthometric } \\
\text { height } \\
\text { (m) }\end{array}$ \\
\hline 1052 & 5.2 & $14672 / 15444$ & 95 & $47 / 48$ & 98 & 0.017 & 0.028 \\
1053 & 5.3 & $13970 / 14253$ & 98 & $41 / 41$ & 100 & 0.011 & 0.026 \\
1054 & 5.6 & $14419 / 14565$ & 99 & $45 / 45$ & 100 & 0.011 & 0.026 \\
1059 & 5.7 & $14613 / 15546$ & 94 & $43 / 44$ & 97 & 0.021 & 0.044 \\
1070 & 6.0 & $16234 / 16911$ & 96 & $36 / 44$ & 82 & 0.019 & 0.063 \\
1071 & 6.3 & $16386 / 16893$ & 97 & $46 / 46$ & 100 & 0.016 & 0.073 \\
\hline
\end{tabular}

Benchmark 1052:

FILE: 10520582.070000085308

NGS OPUS SOLUTION REPORT

$================$

All computed coordinate accuracies are listed as peak-to-peak values. For additional information: http://www.ngs.noaa.gov/OPUS/about.html\#accuracy

USER: bkwoodwa@usgs.gov

RINEX FILE: 10520580.070

SOFTWARE: page5 0612.06 master28.pl

EPHEMERIS: igu14162.eph [ultra-rapid]

NAV FILE: brdc0860.07n

ANT NAME: ASH701975.01AGP NONE

ARP HEIGHT: 2.0
DATE: March 28, 2007

TIME: $17: 42: 51$ UTC
REF FRAME : NAD_83 (CORS96) (EPOCH:2002.0000)

$\begin{array}{lrl}X: & -639505.121(\mathrm{~m}) & 0.019(\mathrm{~m}) \\ \mathrm{Y}: & -4640260.384(\mathrm{~m}) & 0.018(\mathrm{~m}) \\ \mathrm{Z}: & 4314932.810(\mathrm{~m}) & 0.023(\mathrm{~m})\end{array}$

ITRF00 (EPOCH:2007.1580)

$\begin{array}{rr}-639505.839(\mathrm{~m}) & 0.019(\mathrm{~m}) \\ -4640259.072(\mathrm{~m}) & 0.018(\mathrm{~m}) \\ 4314932.739(\mathrm{~m}) & 0.023(\mathrm{~m})\end{array}$




$\begin{array}{rrrr}\text { LAT: } & 42 & 50 & 33.24540 \\ \text { E LON: } & 262 & 9 & 11.22636 \\ \text { W LON: } & 97 & 50 & 48.77364 \\ \text { EL HGT: } & & 350.419(\mathrm{~m}) \\ \text { ORTHO HGT: } & & 376.327(\mathrm{~m})\end{array}$

$0.028(\mathrm{~m})$

$0.020(\mathrm{~m})$

$0.020(\mathrm{~m})$

$0.013(\mathrm{~m})$

$0.028(\mathrm{~m})$

Peak-to-peak indicator of solution uncertainty

$\begin{array}{lcc} & \text { UTM COORDINATES } & \text { STATE PLANE COORDINATES } \\ & \text { UTM (ZOne 14) } & \text { SPC }(2600 \mathrm{NE}) \\ \text { Northing }(\mathrm{Y}) \text { [meters] } & 4743977.316 & 336323.988 \\ \text { Easting }(\mathrm{X}) \quad \text { [meters] } & 594228.876 & 675984.698 \\ \text { Convergence [degrees] } & 0.78416221 & 1.42686494 \\ \text { Point Scale } & 0.99970923 & 0.99993160 \\ \text { Combined Factor } & 0.99965430 & 0.99987665\end{array}$

US NATIONAL GRID DESIGNATOR: 14 TNN9422943977 (NAD 83)

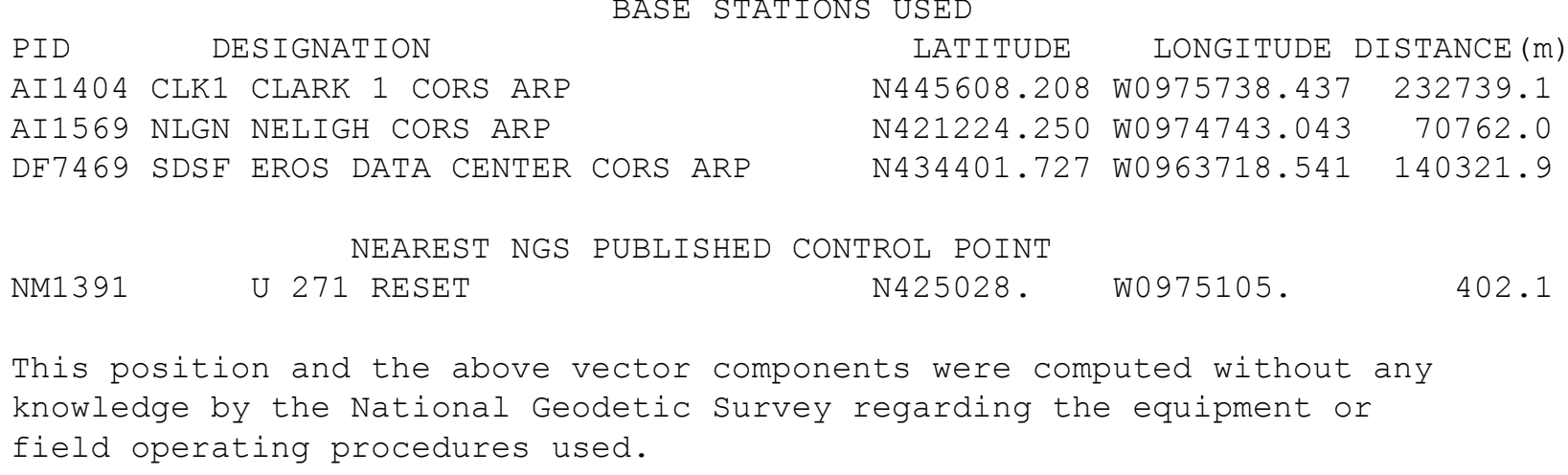

If results did not meet the requirements, the following steps could be taken to potentially improve the solution:

- Manual selection of different CORS stations for reprocessing in OPUS.

- A later submission to OPUS for more precise ephemeris and orbit files.

- Re-occupation for additional observation sessions and re-submission to OPUS.

- Manual editing of the observation file for re-processing $\mathrm{L}_{1}$ frequency (only) data through software.

One way to collect additional data on a benchmark that did not meet the required quality, without delaying Level III single-base RTK fieldwork, is to preliminarily use coordinates obtained from the OPUS results that did not meet the Level II single-base static requirements. These coordinates would be used to establish the base station over the deficient benchmark during the first day of RTK surveying; however, the base receiver would collect static data while objective points are being established by rover. The new static data could be processed through OPUS to potentially obtain better results, so that coordinates from the RTK survey, based on the original solution (that did not meet the requirements), could simply be adjusted once a better solution was achieved. In addition, if the original OPUS solution meets Level II requirements (and assurance of vertical peak-topeak less than or equal to $0.06 \mathrm{~m}$ ), subsequent static data could be collected during the RTK survey and processed to improve the results from a Level II to a Level I single-base static survey (by averaging the two high-quality solutions and readjusting the coordinates collected). This same process of collecting static data during a RTK survey is also useful for checking the stability of benchmarks that are used several years after establishment.

The NGS benchmark Santee Reset is used to provide an assessment or check regarding the quality of single-base static (OPUS) solutions. Static data is collected on the NGS benchmark Santee Reset and processed through OPUS to provide comparison. The OPUS results of the benchmark Santee Reset are provided below. 
Benchmark Santee Reset:

FILE: SANT0861.070 000105396

NGS OPUS SOLUTION REPORT

USER: bkwoodwa@usgs.gov RINEX FILE: sant0860.070

SOFTWARE: page5 0612.06 master12.pl EPHEMERIS: igr14202.eph [rapid] NAV FILE: brdc0860.07n ANT NAME: ASH701975.01AGP NONE ARP HEIGHT: 1.440

REF FRAME: NAD_83(CORS96) (EPOCH:2002.0000)

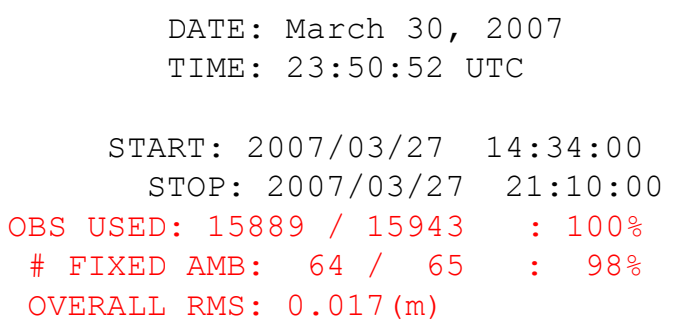

ITRFOO (EPOCH:2007.2349)

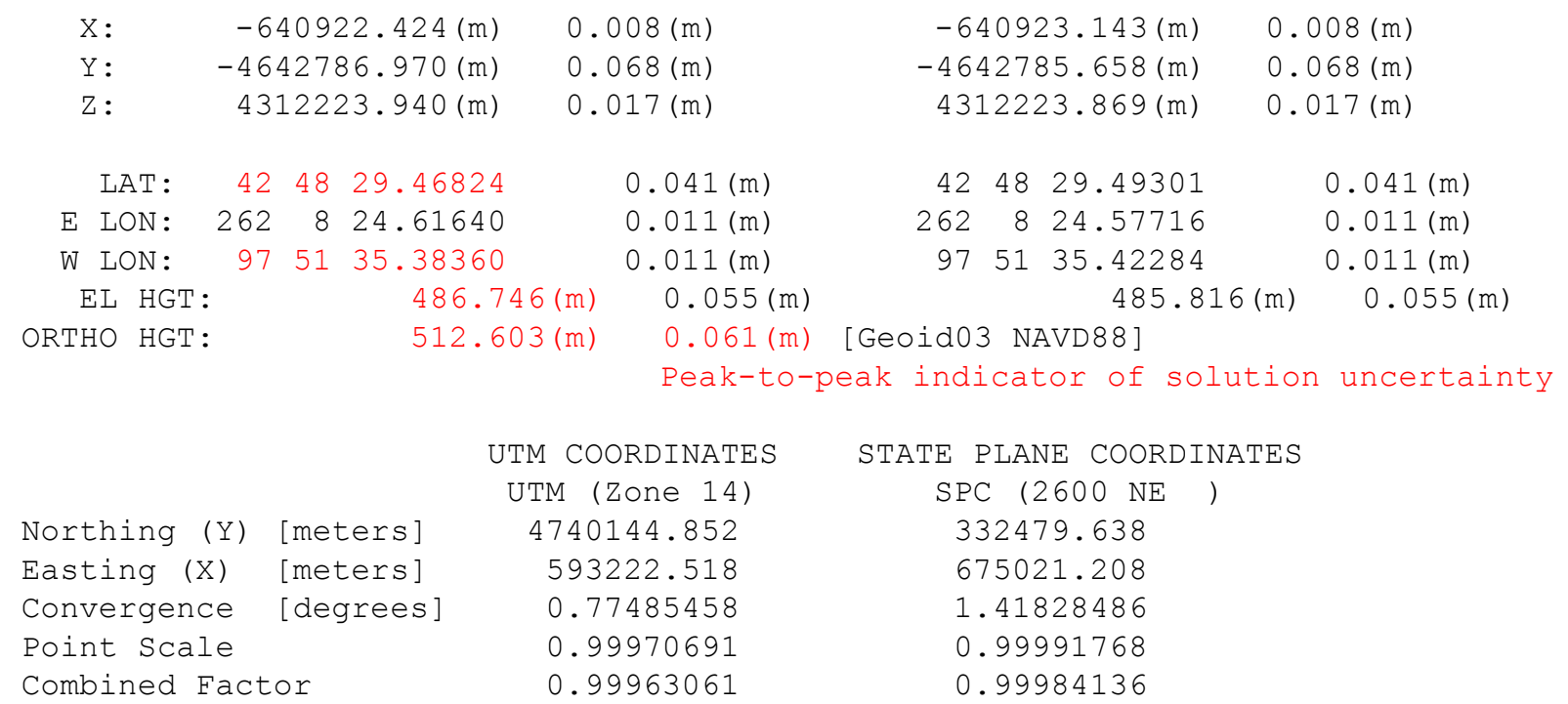

US NATIONAL GRID DESIGNATOR: 14TNN9322340145 (NAD 83)

BASE STATIONS USED

$\begin{array}{lccrrr}\text { PID } & \text { DESIGNATION } & \text { LATITUDE } & \text { LONGITUDE } & \text { DISTANCE (m) } \\ \text { AI1404 } & \text { CLK1 CLARK 1 CORS ARP } & \text { N445608.208 } & \text { W0975738.437 } & 236520.0 \\ \text { AI1569 NLGN NELIGH CORS ARP } & \text { N421224.250 W0974743.043 } & 67026.0 \\ \text { DF7469 SDSF EROS DATA CENTER CORS ARP } & \text { N434401.727 W0963718.541 } & 143796.2 \\ \text { NM1382 } & \text { NEAREST NGS PUBLISHED CONTROL POINT } & & \\ \text { SANTEE RESET } & \text { N424829.468 W0975135.383 } & 0.0\end{array}$

This position and the above vector components were computed without any knowledge by the National Geodetic Survey regarding the equipment or field operating procedures used.

8002 The Opus solution for your submitted RINEX file appears to be 8002 quite close to an NGS published control point. This suggests that 8002 you may have set your GPS receiver up over an NGS control point. 8002 Furthermore, our files indicate that this control point has not 8002 been recovered in the last five years.

8002 If you did indeed recover an NGS control point, we would 8002 appreciate receiving this information through our web based 8002 Mark Recovery Form at

8002 http://www.ngs.noaa.gov/products_services.shtml\#MarkRecoveryForm 
The ending note to the OPUS solution stating that this OPUS solution appeared to be quite close to a NGS published control point was a good indication that the position checked favorably against the published coordinates. In addition, this note provided the website to report the recovery of a benchmark. When the coordinates determined through this OPUS solution were compared to the published coordinates (indicated below in the benchmark datasheet), the OPUS solution differed from the published coordinates as -0.00022 degrees latitude, +0.00001 degrees longitude, +0.007 meters in ellipsoid height, and +0.012 meters in orthometric height (same geoids used in this comparison). Overall, these results indicated that OPUS-derived solutions on this day, and in this area, are giving accurate results.

\section{Static Network Surveying-Datum Establishment for River Survey}

The following is an example of a network survey that was done to establish benchmarks at a study site on the Missouri River in Nebraska to conduct topographic single-base RTK surveys. This network depicts an actual USGS data collection effort that did not meet all requirements of a Level II survey; however, the requirements not met are discussed and suggestions for meeting those requirements are made.

The goal of this network was to establish coordinates at two newly constructed benchmarks (1013 and 1014) at a study site where single-base RTK surveys were to be performed (fig. 4-3).

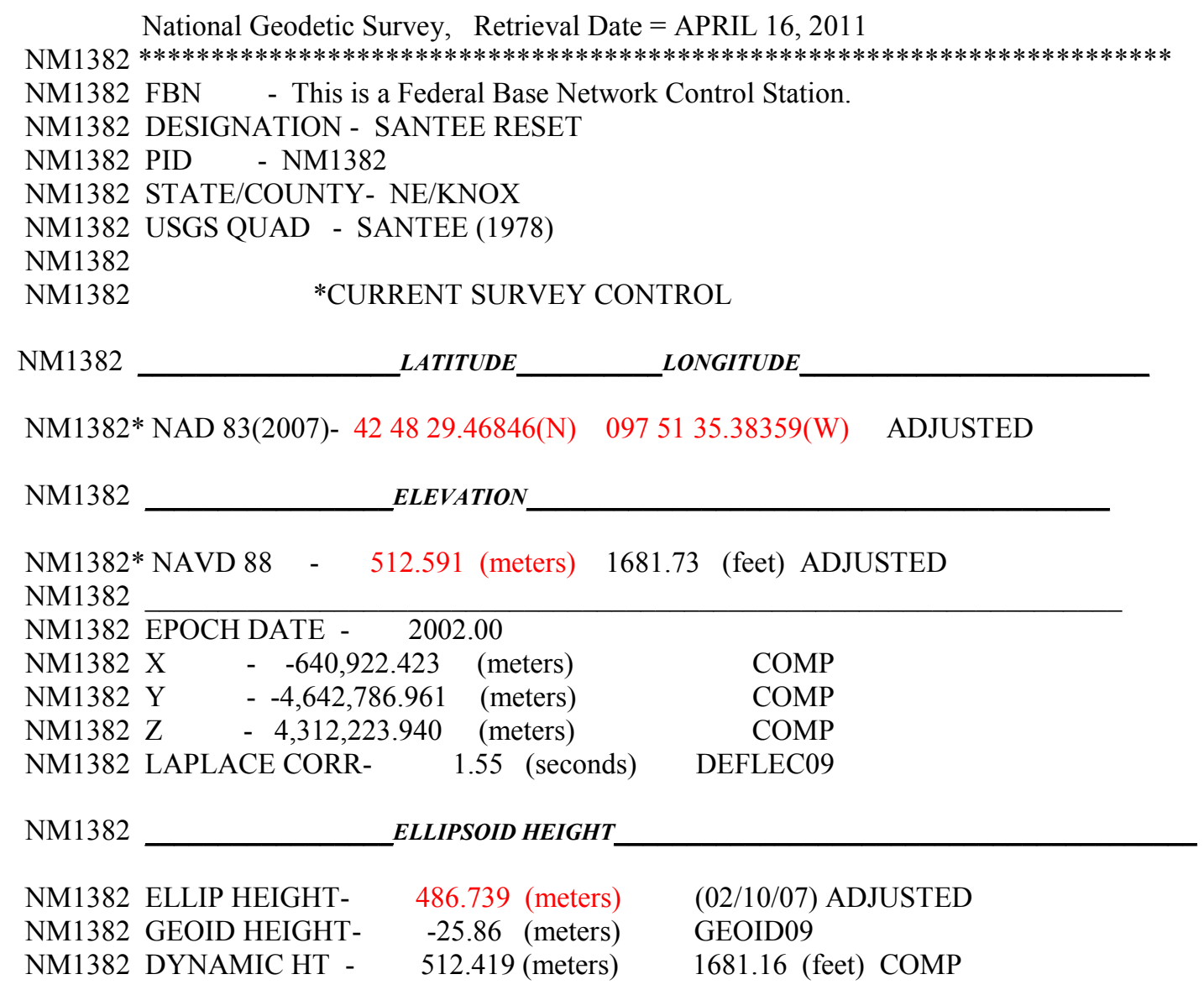

After all benchmarks had been established and coordinates were determined through OPUS processing, Level III RTK surveying began, utilizing two benchmarks in each survey (either as a localization point and a check, or as a base station location and a check). This example indicates the importance of conducting single-base static OPUS methods on benchmarks so that continuity is assured among the local benchmark network and any historic data collection derived from that network. In terms of a localization, this quality check can be performed to verify that the method used is meeting the needs of the survey.
New benchmarks were required to be established as a result of the proximity and quality of existing benchmarks. Benchmark construction and location were derived from topographic and geologic assessments, landowner permission, and field reconnaissance. Once established, 2 new benchmarks were surveyed utilizing 3 NGS benchmarks (PID AE 9294, referred to in the survey as OMA1; PID MK 0365, referred to as TEK; and PID MJ 0984, referred to as MJ84). The location of the project area was along the Missouri River at the Nebraska-Iowa boarder, slightly north of Omaha, Nebraska (fig. 4-3). To fulfill a Level II quality survey, at least one more 


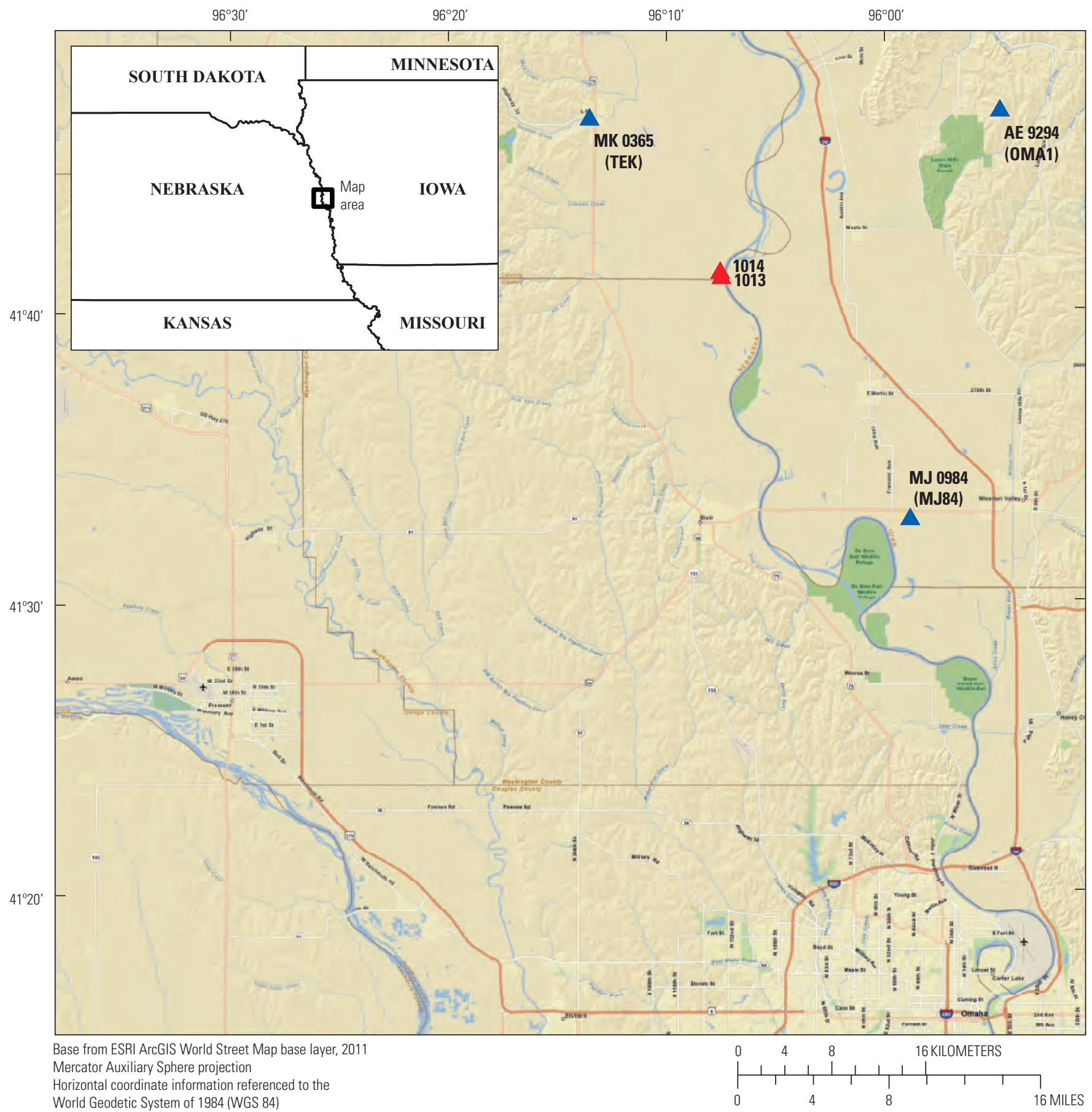

EXPLANATION

National Geodetic Survey (NGS) benchmarks (vertical order II)

1014 Objective points with identifier established using network

Figure 4-3. Objective points established at locations 1013 and 1014 using a static network survey that includes controlling National Geodetic Survey (NGS) benchmarks. 
NGS benchmark should have been included in this network for elevation control, and in most effective practice, a fifth benchmark should have been included to perform a quality check. The network design of this survey was simple: observations occurred at each NGS control benchmark while simultaneously conducting observations on the newly established benchmarks. The time span of each observation can be seen in the top left block in the processing software (fig. 4-4). The time of data collection at benchmark TEK did not meet the 4-hour observation requirement; however, the other two benchmarks exceeded or nearly met the requirements. The observation time at both newly established benchmarks exceeded the 1-hour minimum requirement; however, no objective point or baseline was double occupied to meet the re-observation requirements. The spacing of this network was adequate for a Level II network survey, which requires objective point and control benchmark spacing to be located within $25 \mathrm{~km}$ of each other. In addition, Level II spacing was satisfied because the control benchmarks were within $60 \mathrm{~km}$ of each other (fig. 4-3).

Mission planning was undertaken as observation days were evaluated for satellite availability, and PDOP and nearby CORS data were evaluated for stability and data availability. The field campaign involved the following assurances:

- Benchmark evaluation, reconnaissance, field preparation, and data storage.

- Bubble check and calibration of base fixed-height tripod and rover bipod.

- Base fixed-height or dual-clamped tripod stabilized by chains or sandbags.

- Recovery, establishment, and observation forms used to document existing or established benchmarks (appendixes 1 and 2).

During data collection, GNSS receivers were set up on fixed-height tripods. Detailed notes were taken by the field crew, including information required in appendixes 1 and 2 . To re-observe objective points (the newly established benchmarks), the receivers collecting data could have been stopped for a short time, the tripods repositioned over the benchmarks, and powered up again to continue collecting data as a new observation; although the time required to collect 2 short static sessions at each objective point would be similar to collecting 1 longer static session, 2 short sessions would aid in detecting errors when processing the network.

Once the field data collection was completed, processing began by checking each data set. Data were converted to RINEX format for easy review in a text editor software (NotePad, WordPad, or TextPad) or just loaded into a processing software. Before entering data files into a processing software, a new project was created. Settings such as the coordinate system, measurement units, and other project details were entered. In addition, most processing software will allow a desired accuracy that will be used by the software to determine if statistical tests pass or fail. The desired accuracy for this network survey was set to: Horizontal $0.02 \mathrm{~m}+1 \mathrm{ppm}$ and Vertical $0.04 \mathrm{~m}+2 \mathrm{ppm}$. When data were brought into the processing software, the receiver antenna type was defined and the receiver antenna height entered correctly (it is most effective to enter vertical height, but if a non-fixed height tripod is used, some software packages allow the user to define the antenna height as a slant height, as illustrated in appendix 2, and noted as a menu item under "Height Type" in fig. 4-4).

The next step in processing the static network was to process all baselines. Ten baselines were processed in this Missouri River network. The second step offered in most processing software is blunder detection, which is used to detect whether or not a data set does not fit well with the other data, possibly because the wrong name, receiver antenna height, or receiver antenna type has been entered. No blunders were detected within the Missouri River network (fig. 4-5). If blunders were detected, an investigation into the cause and correction of the problem site should have been done according to guidance given for each processing software. Next, processed baselines were reviewed for quality, including re-observed baselines, and baselines of poor quality were either evaluated to determine and correct the cause of the poor results or were not used in the network adjustment. The quality of 10 baselines processed in the Missouri River network were described in terms of the change in horizontal, vertical, and up position (delta $\mathrm{x}, \mathrm{y}, \mathrm{z}$ ) of the developed baseline between 2 points, the error reflecting the change in $\mathrm{x}, \mathrm{y}$, and $\mathrm{z}$ positions at 2 sigma (95-percent confidence), and determination of the baseline length (fig. 4-6). At this point in a network adjustment, no observations were held to a fixed position, and as a result, all observations ( $\mathrm{x}, \mathrm{y}$, and $\mathrm{z}$ positions) could be adjusted slightly.

In the Missouri River network example, the poorest quality baseline was MJ84 to TEK based on the 95-percent error estimate (fig. 4-6). This baseline was de-selected, and the network was evaluated to ensure connectivity among all objective points and benchmarks. Also, re-observed baselines were reviewed at this time. If re-observed baselines indicated similar results, leaving both in the network would not have been a problem; however, if they did not have similar results, the baseline reflecting the poorest quality (greatest 95 -percent error estimate) should have been removed. From 10 initial baselines evaluated in this network, 6 baselines were selected to be used for minimally and fully constrained adjustments. Note that some network processing guidance describes completing network adjustments using only independent baselines (or in simple terms only processing 2 sides of each triangle in the network because the third side is dependent on the results of the first 2). Through practice it has been indicated that processing a network using all baselines (opposed to independent baselines) has a negligible effect on the final results, so the time required to select the independent baseline may not be needed; however, the surveyor should use judgment regarding baseline selection and guidance given for the processing software used. 


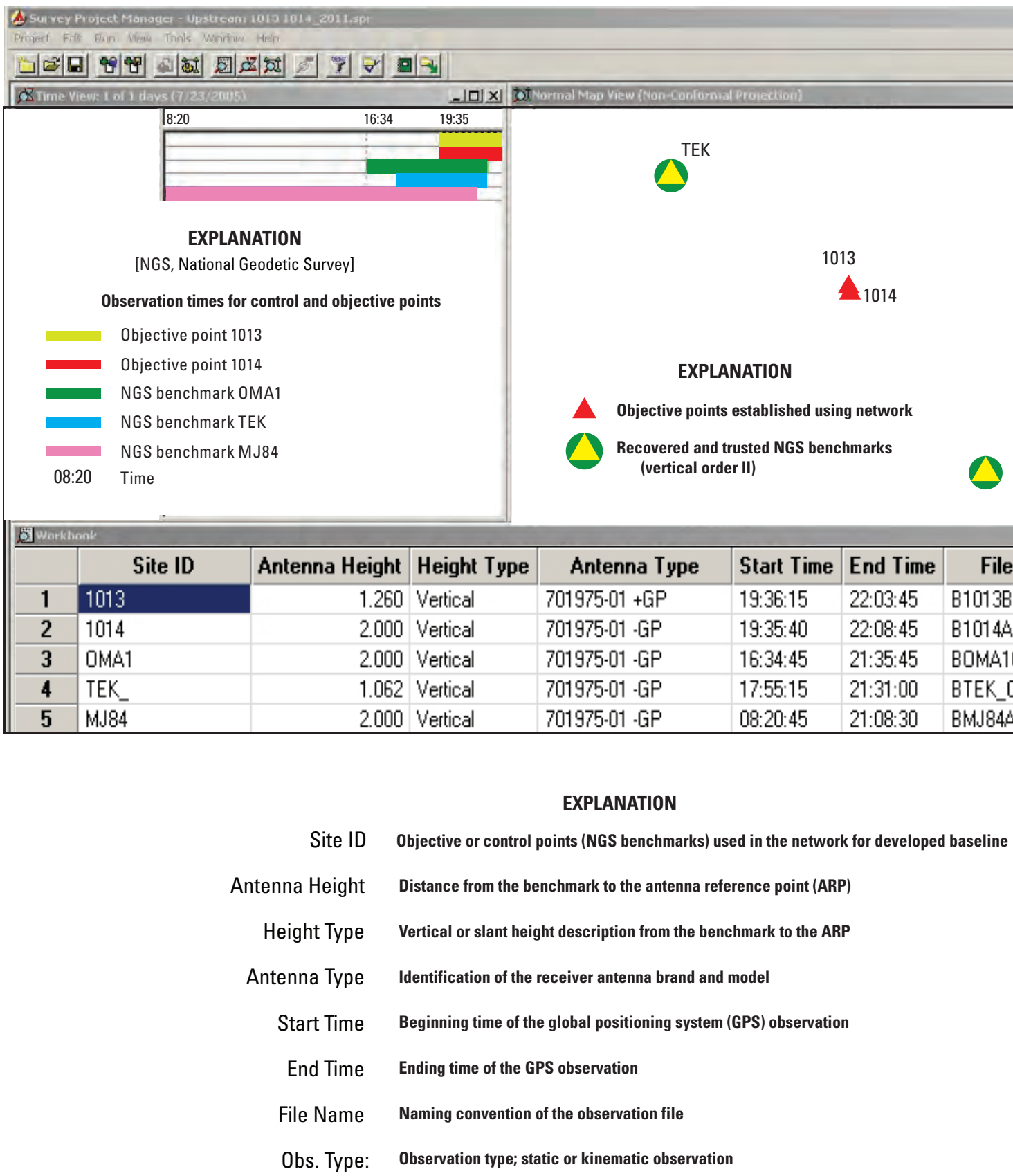

Figure 4-4. Adjustment software depicting Global Navigation Satellite System receiver data file information and location; including antenna type and height, and observation times. 


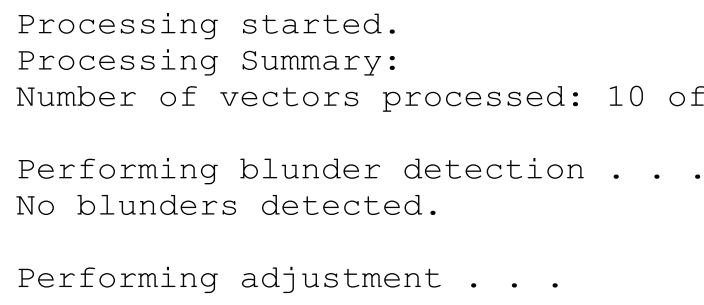

FILLNET - Ashtech Network Adjustment Package Copyright () 2002 Thales Navigation. All rights reserved. Software program used for the adjustment Sunday, July 24, 2005 06:10:51 PM
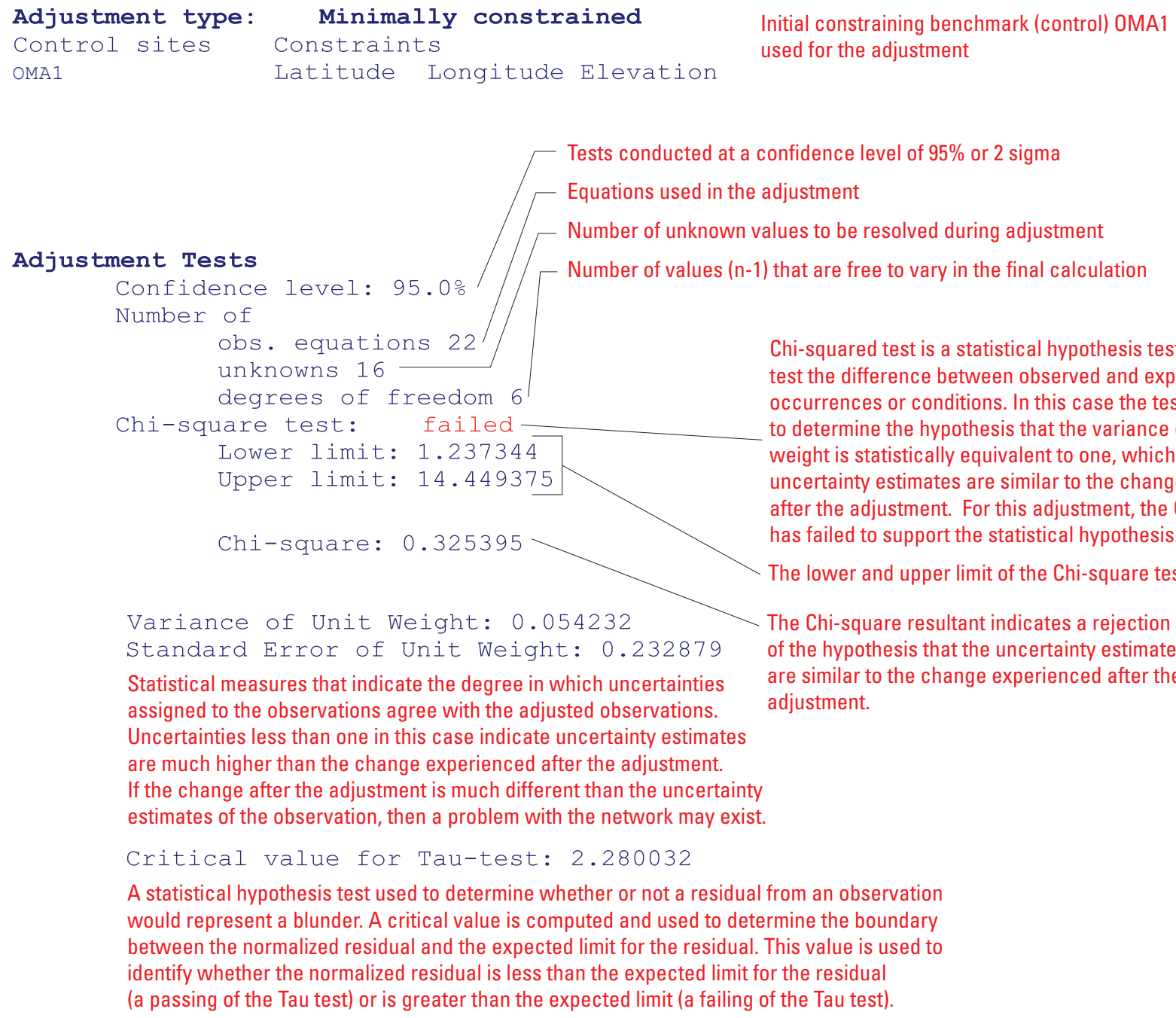

Figure 4-5. Network adjustment output representing initial processing steps and a minimally constrained adjustment of the Missouri River network. 


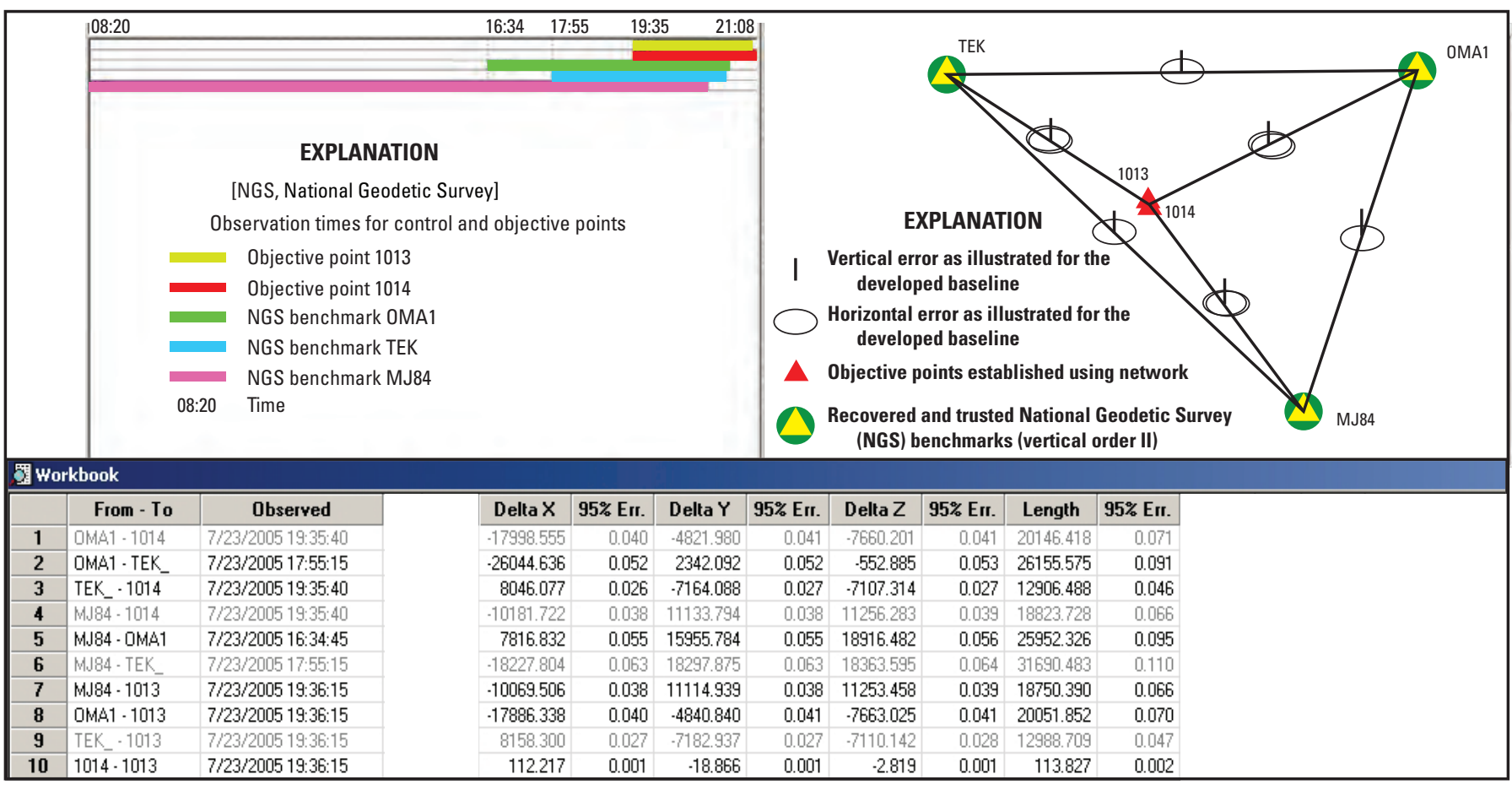

EXPLANATION

\begin{tabular}{|c|c|}
\hline From - To & Objective or control points (NGS benchmarks) used in the network for developed baseline \\
\hline OMA1 - 1014 & Grey color denotes deselected baseline \\
\hline Observed & Date and time of observation \\
\hline Delta $x$ & Change in the horizontal position, in meters, for the developed baseline \\
\hline Delta y & Change in the vertical position, in meters, for the developed baseline \\
\hline Delta z & Change in the height or "up" position, in meters, for the developed baseline \\
\hline $95 \%$ Err. & $\begin{array}{l}95 \text { percent error; error, in meters, (at } 2 \text { sigma or } 95 \text { percent confidence) representing } \\
\text { the developed baseline as a "best fit" for the network }\end{array}$ \\
\hline Length & Baseline length, in meters \\
\hline
\end{tabular}

Figure 4-6. Adjustment software package depicting processed and deselected baselines in the Missouri River network.

After all baselines were evaluated (and reprocessed if changes were made), the coordinates for all known benchmarks in the network should be entered regardless of whether or not the benchmark will be used as control when processing or as a quality check. The NGS datasheets for three benchmarks included in the Missouri River network were downloaded from the NGS website and coordinates were entered into the software program. For the minimally constrained adjustment, only one benchmark was held as a fixed control [note selection OMA1 held as a fixed position as indicated by "Hor/Ver" in the "Fixed" column (fig. 4-7)].

The results from the minimally constrained adjustment indicated that the first step this software took in adjusting a network was to check network connectivity (fig. 4-5). The connectivity test passed, identifying 5 benchmarks and 6 baselines to be included in the adjustment. If the test did not pass, the user would have to re-evaluate which baselines were selected, and potentially re-adjust to ensure all benchmarks were connected among baselines chosen. The results from the minimally constrained adjustment indicated that all baselines were adjusted with small standard residuals (fig. 4-8). The standard residual is a small correction that is applied to the observation to obtain the best fit of all observations.

Processing results from network adjustments involves statistical tests that provide an indication of the quality of the network solution.

A successful least squares adjustment is one where observations are changed as little as possible, and the amount of change to any one observation is within expected levels or about the same magnitude as the uncertainty in the observations (Ashtech Precision Products, 2001). The relation between uncertainties assigned with observations and the magnitude of change in the adjustment is monitored by the variance of unit weight and the standard error of unit weight. The standard error of unit weight is the square root of the variance of unit weight. Both of these statistical measures indicate the degree in which uncertainties assigned to the observations agree with the adjusted observations.

If the uncertainty estimate assigned with the observation is similar to the change of each observation during the 


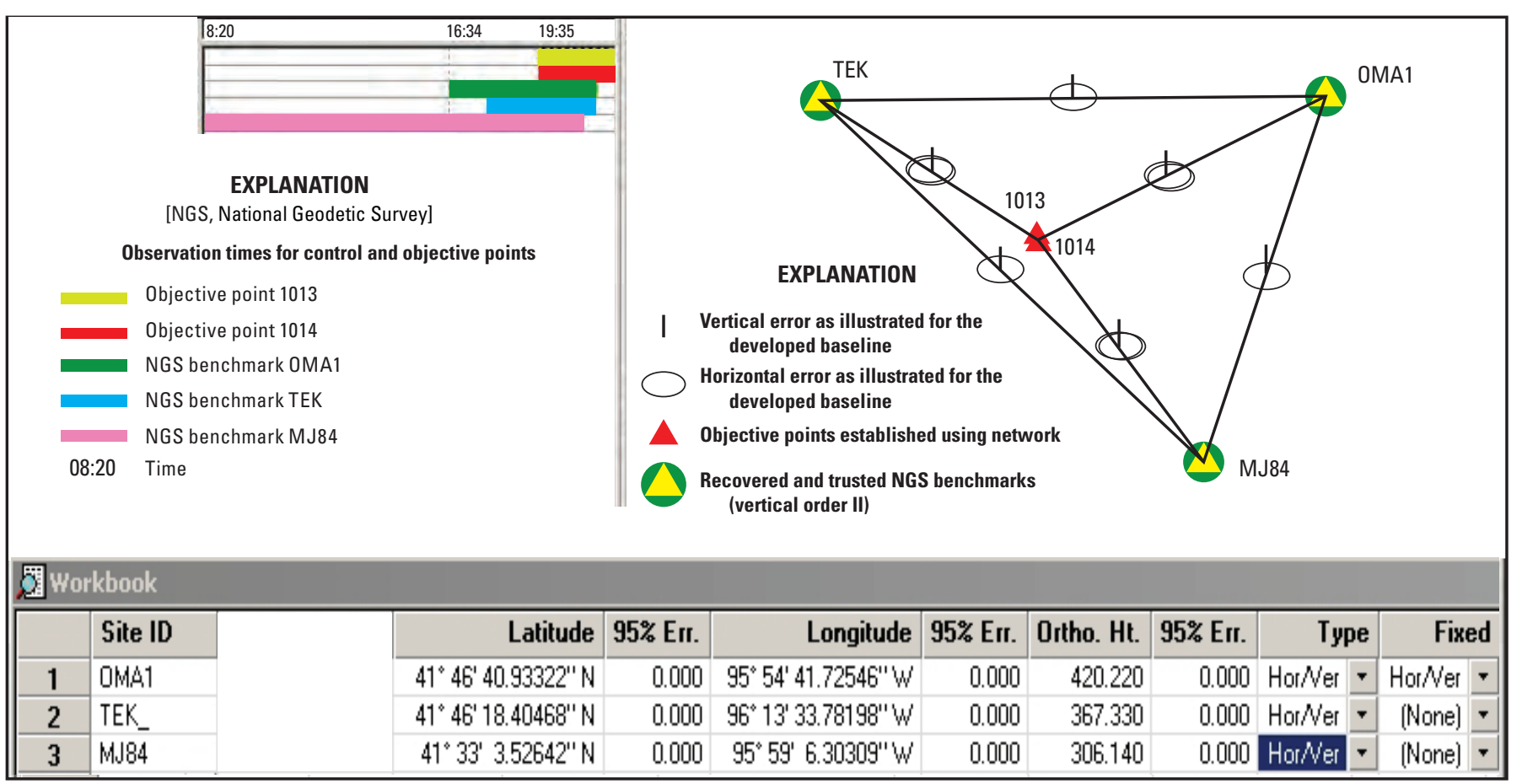

\section{EXPLANATION}

\begin{tabular}{|c|c|}
\hline Site ID & Control points (NGS benchmarks) used in the network \\
\hline Latitude & Horizontal position of the control point (NGS benchmark) \\
\hline Longitude & Vertical position of the control point (NGS benchmark) \\
\hline $95 \%$ Err. & $\begin{array}{l}95 \text { percent error; error, in meters, (at } 2 \text { sigma or } 95 \text { percent confidence) } \\
\text { representing the developed baseline as a "best fit" for the network }\end{array}$ \\
\hline Ortho. Ht. & Orthometric height; elevation of the control point (NGS benchmark) \\
\hline Type & Represented position of the benchmark: horizontal, vertical or both \\
\hline Fixed & Identification of constrained control point (NGS benchmark) \\
\hline
\end{tabular}

Figure 4-7. Adjustment software depicting coordinates entered for National Geodetic Survey (NGS) benchmarks and one benchmark (OMA 1) held as fixed (horizontally and vertically) for the minimally constrained adjustment. 


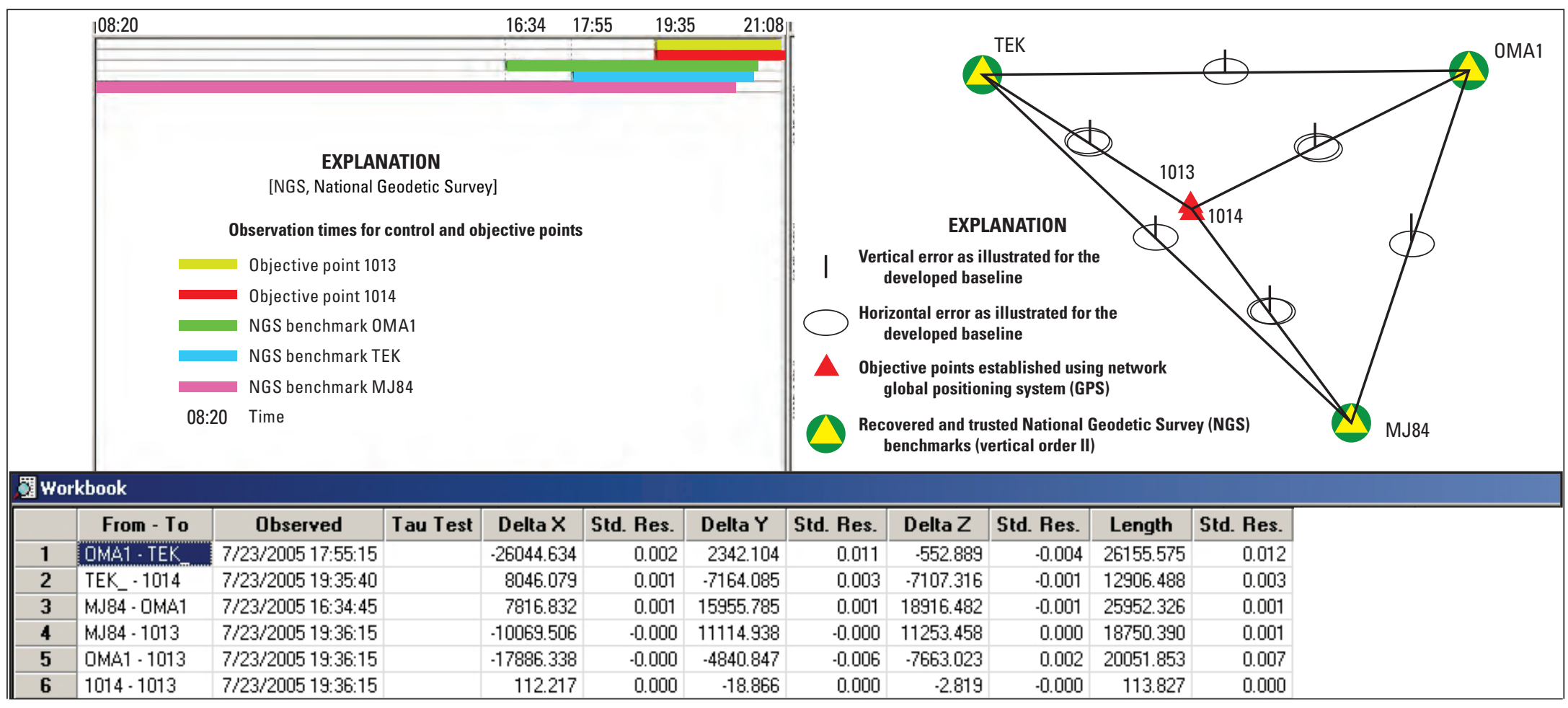

\section{EXPLANATION}

From -To Objective or control points (NGS benchmarks) used in the network for developed baseline

Observed Date and time of observation

Tau Test Determines if a standard residual is unexpectantly large. "Fail" appears if the test did not pass. Blank represents the test passing

Delta $X$ Change in the horizontal position, in meters, for the developed baseline

Delta $Y \quad$ Change in the vertical position, in meters, for the developed baseline

Delta Z Change in the height or "up" position, in meters, for the developed baseline

Std. Res. Standard residual; differences between the delta $x, y$, and $z$ components and length of the observed baseline and adjusted baseline

Length Baseline length, in meters

Figure 4-8. Adjustment software package depicting results of the minimally constrained network adjustment. 
adjustment, then the variance of unit weight will be approximately one. This essentially indicates how well the data fit the adjusted network. If the uncertainty estimates are much higher than the change experienced after the adjustment, then the values will be less than 1 , and if the uncertainty estimates are too low as compared to the change after the adjustment, then the values will be greater than 1 . If the change after the adjustment is quite different than the uncertainty estimates of the observation, then a problem with the network may exist, or uncertainty estimates may not be realistic. Because there are many factors affecting the uncertainty in each observation, uncertainty estimates are not easy to determine. Uncertainty estimates can be derived from past experience of resultant accuracies in network adjustments as well as the base error of the equipment manufacture plus an allowable part-per-million (Brinker and Minnick, 1995). Many statistics completed in a least squares adjustment utilize uncertainty estimates, and most adjustment programs use the variance and standard error of unit weight to adjust all other statistics; however, most processing software, including Ashtech Solutions, automatically compensates for unrealistic error estimates, so that problems that potentially arise from over- or under-estimated uncertainty are often non-existent. The Chi-squared test is a statistical hypothesis test used to test the difference between observed and expected occurrences or outcomes. The test is used by Ashtech Solutions software to determine the hypothesis that the variance of unit weight is statistically equivalent to one, which indicates uncertainty estimates are similar to the change experienced after the adjustment.

The variance of unit weight and the standard error for the minimally constrained adjustment of the Missouri River network were both substantially less than one, as indicated by the failed Chi-squared test (fig. 4-5); however, because the processing software automatically compensates for observation uncertainties that are too high or too low, the passing or failure of the Chi-squared test has no true bearing on the quality of the adjustment (Ashtech Precision Products, 2001).

The least squares adjustment applies small corrections to each observation until the network "fits" together. The solution that fits most optimally is the one that produces the smallest corrections to all observations. These small corrections are termed standard residuals. Small standard residuals usually account for random error in each observation, whereas large standard residuals typically represent a blunder in the dataset. The complicating factor in assessing standard residuals is that residuals (and random error) increase with increasing baseline length. Review of the residuals from the Missouri River minimally constrained network adjustment indicated that all residuals are small (fig. 4-8). The magnitude of standard residuals alone does not provide a full qualitative assessment of the adjusted observations. If a network adjustment produces a large standard residual, there are many potential causes that should be evaluated to determine if the large residual is acceptable. If a large standard residual results from observation uncertainties because of poor satellite coverage and short observation times, then large residuals may not be acceptable and additional observations are needed; however, if a large standard residual is independent of the adjustment, then an assessment of other contributors, such as long baselines (that contribute to the residual), may be deemed inconsequential and stand as correct, and the calculated position held as true.

Most software programs provide a statistical test, such as the Tau test, to determine if a standard residual is unexpectedly large. The observations in the Missouri River network passed the Tau test (fig. 4-8). If an observation had large standard residuals and did not pass the Tau test, the observation should be examined for errors and corrected (receiver antenna height and type, removal of individual satellites, and the time span of the observation to be adjusted); however, after verification that the receiver antenna type and height were entered correctly, and assurance that errors resulting from all other steps of the adjustment were acceptable, then the position may be acceptable. The Tau test provides an indicator for blunders, and it should be understood that a blunder in one observation usually affects residuals in other observations. That is why it is important to examine all observations closely- because an indicated Tau test failure among one pair ("From-To", fig. 4-8) of observations does not necessarily preclude blunders from occurring in other observations.

Once all blunders or low quality vectors have been removed from the minimally constrained adjustment, the fit of control points can be examined. Two NGS benchmarks not held fixed (TEK and MJ84) in the minimally constrained adjustment were shifted slightly from their observed positions to fit the minimally constrained network. Comparing these adjusted coordinates to the published coordinates of the benchmarks can help determine if an observation at one of the benchmarks is of poor quality or if it simply does not fit well with the other benchmarks and should not be used as control in the network. In the Missouri River network, NGS benchmark TEK had the largest misclosure error (difference between the observed/shifted coordinates to published coordinates) but did pass the quality assurance (QA) test according to the specifications given for this project (fig. 4-9).

Finally, the fully constrained network adjustment can be performed once all quality checks meet the project requirements and all blunders or poor observations have been removed. Before running the adjustment, the other NGS benchmarks need to be set as fixed control in the network [in this case there are only 2 other benchmarks (TEK and MJ84), but in a typical Level II survey, there should be at least 3 more elevation control points (for a total of 4) to set as fixed]. After the fully constrained network has been adjusted, the same quality-control statistics must be evaluated to determine if the final adjustment meets the project requirements. The control tie will only show the control points that were not held fixed in the network, but will provide a sense of the final adjustment quality by comparing shifted positions to published positions. In addition, the uncertainty of each adjusted coordinate is described by the difference in delta $\mathrm{x}, \mathrm{y}$, and $\mathrm{z}$ at the 95-percent confidence level (fig. 4-10). In the Missouri River network, the two newly established benchmarks had an 


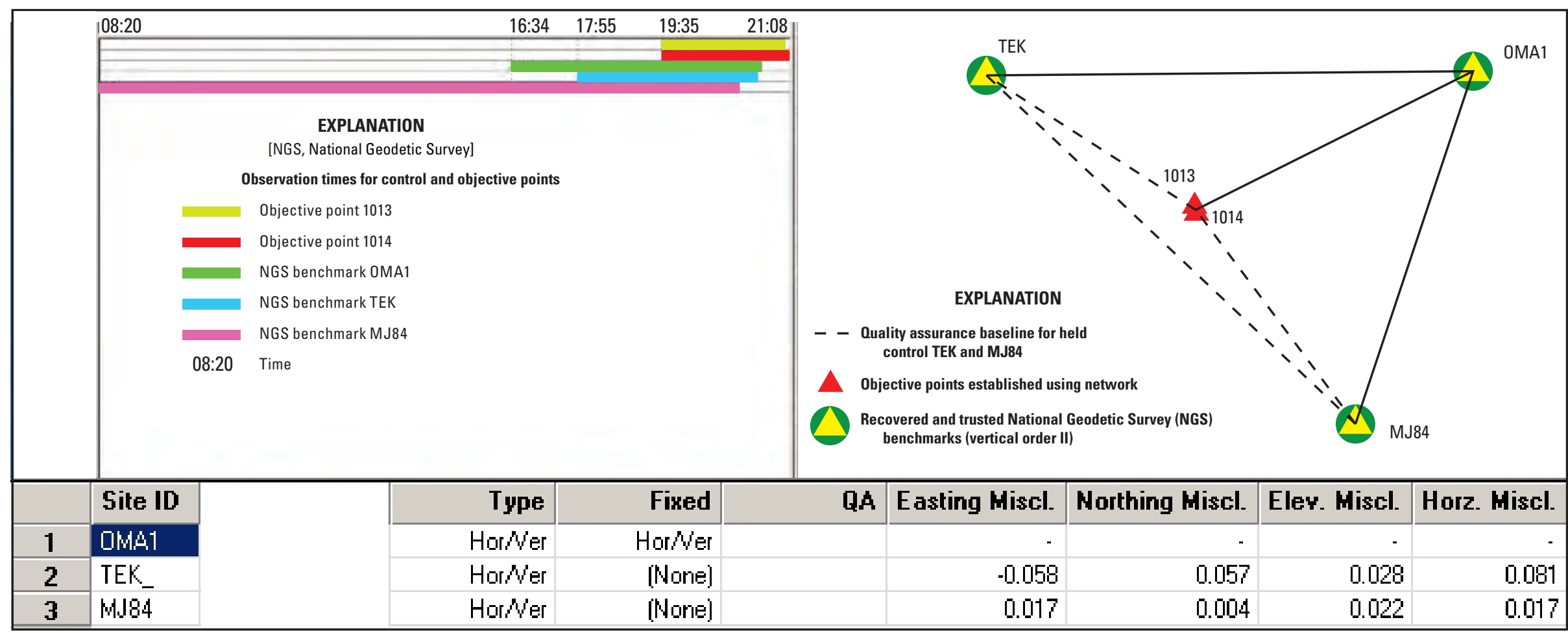

\section{EXPLANATION}

Site ID Control points (NGS benchmarks) used in the network for developed baseline

QA Quality assurance test based on project specifications. "Fail" appears if the test did not pass. Blank represents the test passing

Easting Miscl. Easting misclosure; numerical difference, in meters, between observed and shifted easting coordinate values

Northing Miscl. Northing misclosure; numerical difference, in meters, between observed and shifted northing coordinate values

Elev. miscl. Elevation misclosure; numerical difference, in meters, between observed and shifted elevation coordinate values

Horz. Miscl. Horizontal misclosure; numerical difference, in meters, between observed and shifted horizontal distance values

Figure 4-9. Adjustment software depicting control tie information and quality-assurance test from the minimally constrained Missouri River network adjustment. 


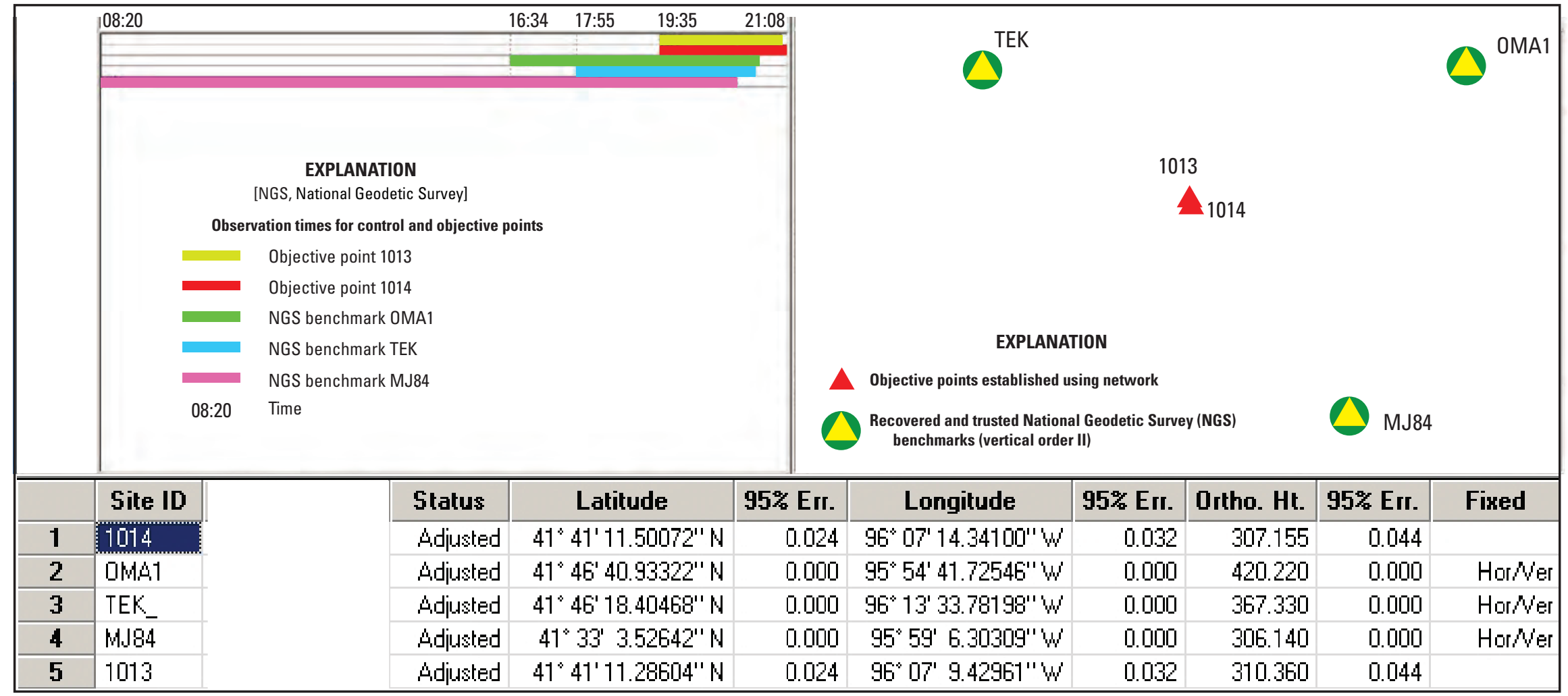

\section{EXPLANATION}

\begin{tabular}{|c|c|}
\hline Site ID & Control points (NGS benchmarks) used in the network \\
\hline STATUS & Status of the network adjustment \\
\hline Latitude & Horizontal position of the control point (NGS benchmark) \\
\hline ongitude & Vertical position of the control point (NGS benchmark) \\
\hline $95 \%$ Err. & $\begin{array}{l}95 \text { percent error, error, in meters, (at } 2 \text { sigma or } 95 \text { percent confidence) } \\
\text { representing the developed baseline as a "best fit" for the network }\end{array}$ \\
\hline Jrtho. & Orthometric height; elevation of the control point (NGS benchmark) \\
\hline Fixed & Identification of constrained control point (NGS benchmark) \\
\hline
\end{tabular}

Figure 4-10. Adjustment software depicting coordinates for the newly established benchmarks resulting from the Missouri River final network adjustment. 
elevation uncertainty of $4.4 \mathrm{~cm}$ at the 95 -percent confidence level; however, without any redundant observations (repeat vectors) and without any NGS benchmarks to be used at quality checks, the real quality of this network is challenging to assess.

Other blunder detection and network quality tools not discussed in this example include loop closure analysis and repeat vector analysis. These are common utilities available within most software. During a loop closure analysis, each possible loop will be processed within the network to determine a closure error. This information can be reviewed to help locate problem vectors and observations. Also, most software calculates repeat vector differences, which can be reviewed to determine if the repeat vectors show consistency, or whether the quality of each vector needs to be examined or potentially removed from the network.

The most important step in processing a static survey network is to understand the software being used and the quality-control statistics. In summary, the quality of the network adjustment can be fully understood through different quality checks run by the software and through observations of benchmarks that are not used as control in the network.

\section{Static Network Surveying—Using OPUS- Projects for Datum Establishment for Terrestrial Imaging and Streamgages}

As referred to earlier in the "Single-Base: Online Positioning User Service (OPUS)" section, OPUS-Projects is a web-based utility that improves efficiency and reduces errors in network processing. OPUS-Projects begins with the creation of a project, then proceeds to data set processing using OPUS-S, uploads the data to the project, calculates network session solutions and ultimately a network adjustment. Once the data have been adjusted and reviewed, the data and results of the project have the option of being published.

Static network processing in OPUS-Projects differs from typical static network processing because of a few distinct steps. OPUS-Projects begins with high-quality OPUS-S solutions. From the data used in these OPUS-S solutions, OPUS-Projects creates sessions from each group of data that spans similar time. Each session is processed individually. In session processing, the user selects which marks and baselines to process and which ones to constrain horizontally, vertically, or three dimensionally (3D). The constraints set in the session solutions do not affect the final network adjustment. Results of the session solutions can be evaluated to detect poor quality data by reviewing the statistical results of the solution and by comparing the results from each session. Several different solutions can be created for each session, including different marks, baselines, and constraints so that the most effective solution can be determined. The session-processing step acts as blunder detection such that once acceptable solutions have been located for all sessions, the session solutions can be merged with new constraints chosen to adjust all marks together into one large network. The interlinking of these sessions through network adjustments increases accuracy (Schenenwerk, 2011).

As an example of using OPUS-Projects, consider a terrestrial-based laser scan that was performed in southwestern Missouri. The scan included a local railroad bridge requiring a couple of control points to geo-rectify the scan with horizontal and vertical assurance. In addition, datum needed to be established at two streamgages in the area.

Mission planning was undertaken as observation days were evaluated for satellite availability, and PDOP and nearby CORS data were evaluated for stability and data availability. The field campaign involved the following assurances:

- Benchmark evaluation, reconnaissance, field preparation, and data storage.

- Bubble check and calibration of base fixed-height tripod.

- Base fixed-height tripod or dual-clamped tripod stabilized by chains or sandbags.

- Recovery, establishment, and observation forms used to document existing or established benchmarks (appendixes 1 and 2).

Level II criteria were applied to a small static network survey approach for the surrounding area. Initially, benchmarks $1760,2216,2385$, and 4430 were selected as exterior points to be used within the network. These benchmarks were within $60 \mathrm{~km}$ of each other and the interior objective points were within $25 \mathrm{~km}$ of the surrounding benchmarks and neighboring objective points. After creating the project using OPUS-Projects, observation metadata were uploaded into the project using an editable mock-up of the datasheets for each mark, which is shown in figures 4-11 and 4-12. The datasheet mimics similar information recovered from the NGS IDB and includes observation files as well as GNSS receiver model and antenna information, firmware, and antenna height. After the data had been uploaded, OPUS-Projects populated a list of available CORS stations to integrate into the network and segregate sessions, in this example day 1 (2012-026-A) and day 2 (2012-027-A) (fig. 4-13). The developed network in figure 4-13 incorporated a CORS station that exceeded baseline lengths required for the Level II survey; however, removing exterior CORS and reducing baseline lengths to the originally prescribed Level II criteria allowed only one CORS station to be utilized among the exterior benchmarks and interior objective points. For this example, however, exterior CORS (exceeding the baseline length of the Level II criteria) were used to demonstrate the effectiveness of utilizing additional CORS stations.

Session processing was performed for both days (day 1 and day 2). The session processing dialog (fig. 4-14) ensured all CORS stations were constrained as horizontal and vertical (3D). Recall that all marks (benchmarks and objective points) were processed through OPUS-S initially and values from those solutions were used as initial coordinates for the projects marks. 


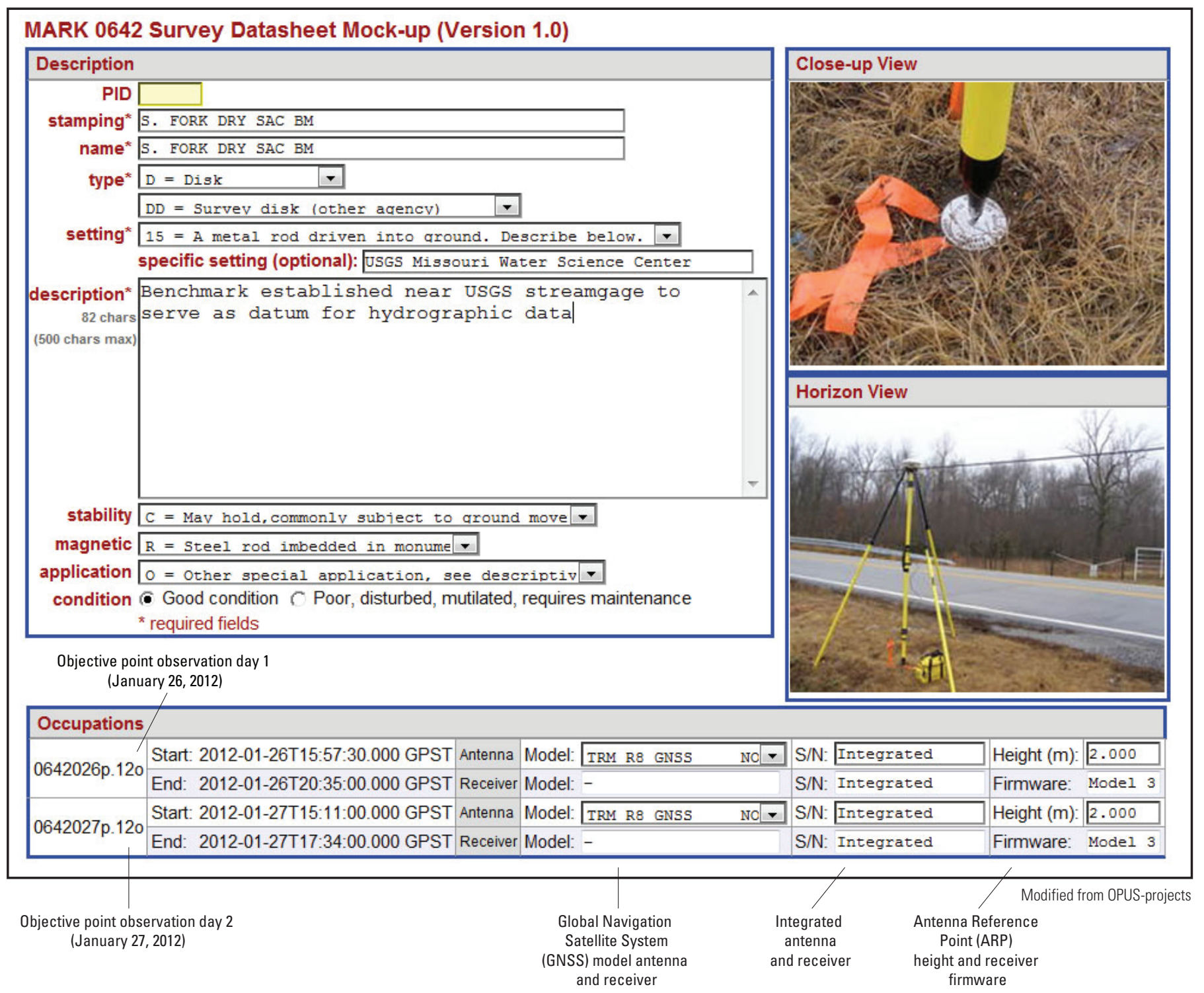

Figure 4-11. An editable mock-up survey datasheet within the Online Position User Service (OPUS) Projects web-based utility identifying an objective point established at a local streamgage. 


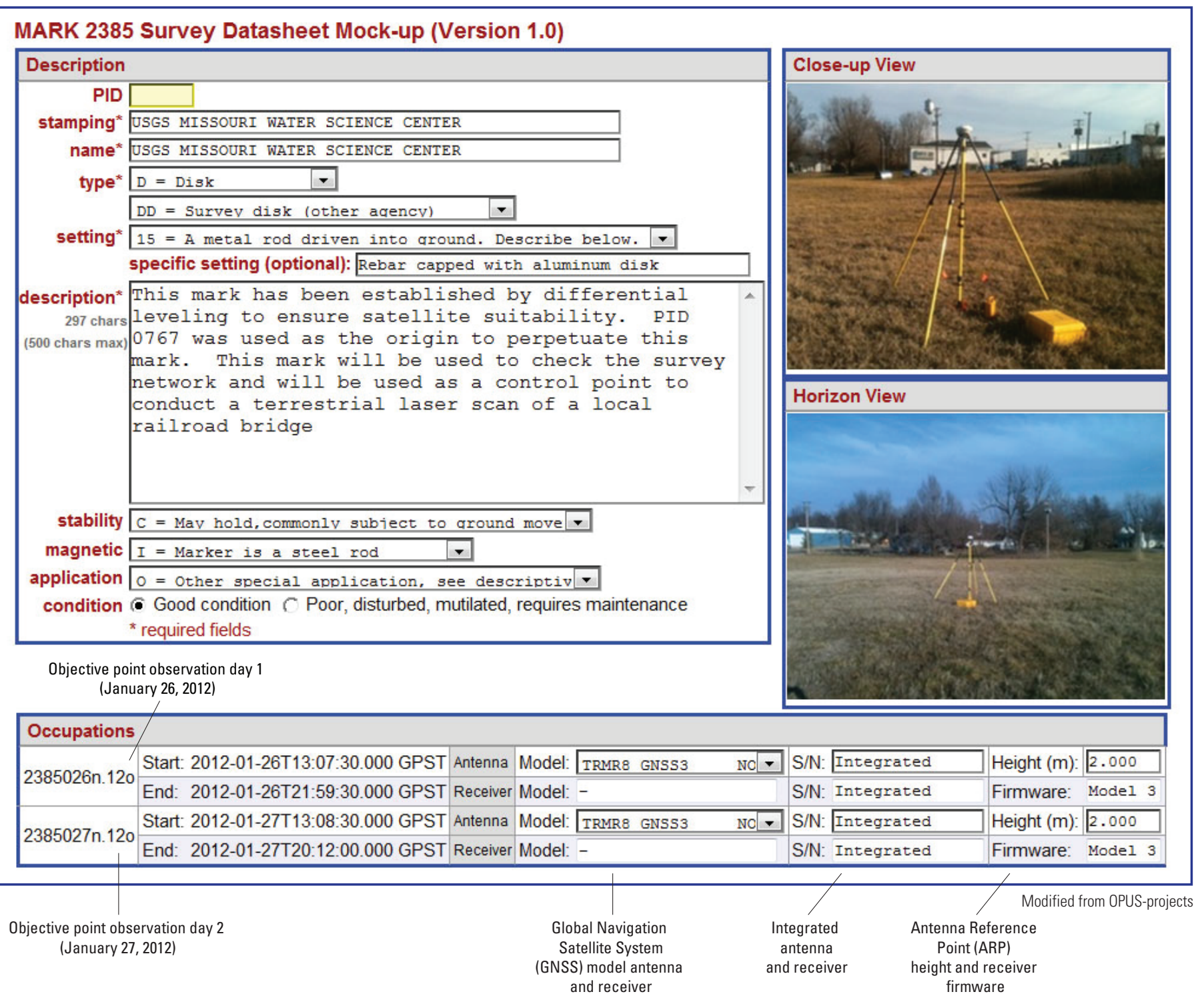

Figure 4-12. An editable mock-up survey datasheet within the Online Position User Service (OPUS) Projects web-based utility identifying an objective point established as a ground control point for a terrestrial laser scan. 


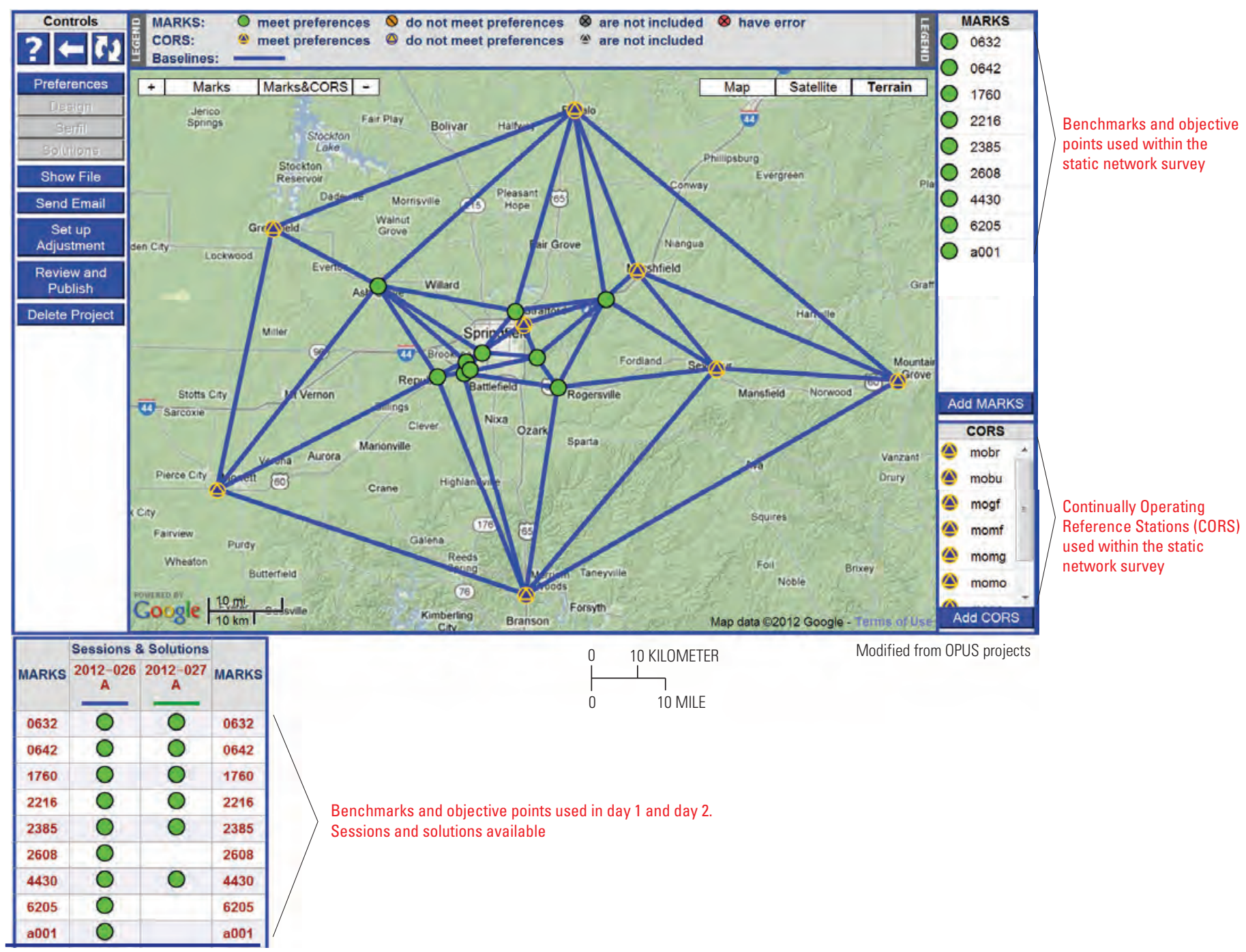

Figure 4-13. Static survey network developed within Online Position Users Service (OPUS) projects web-based utility. 


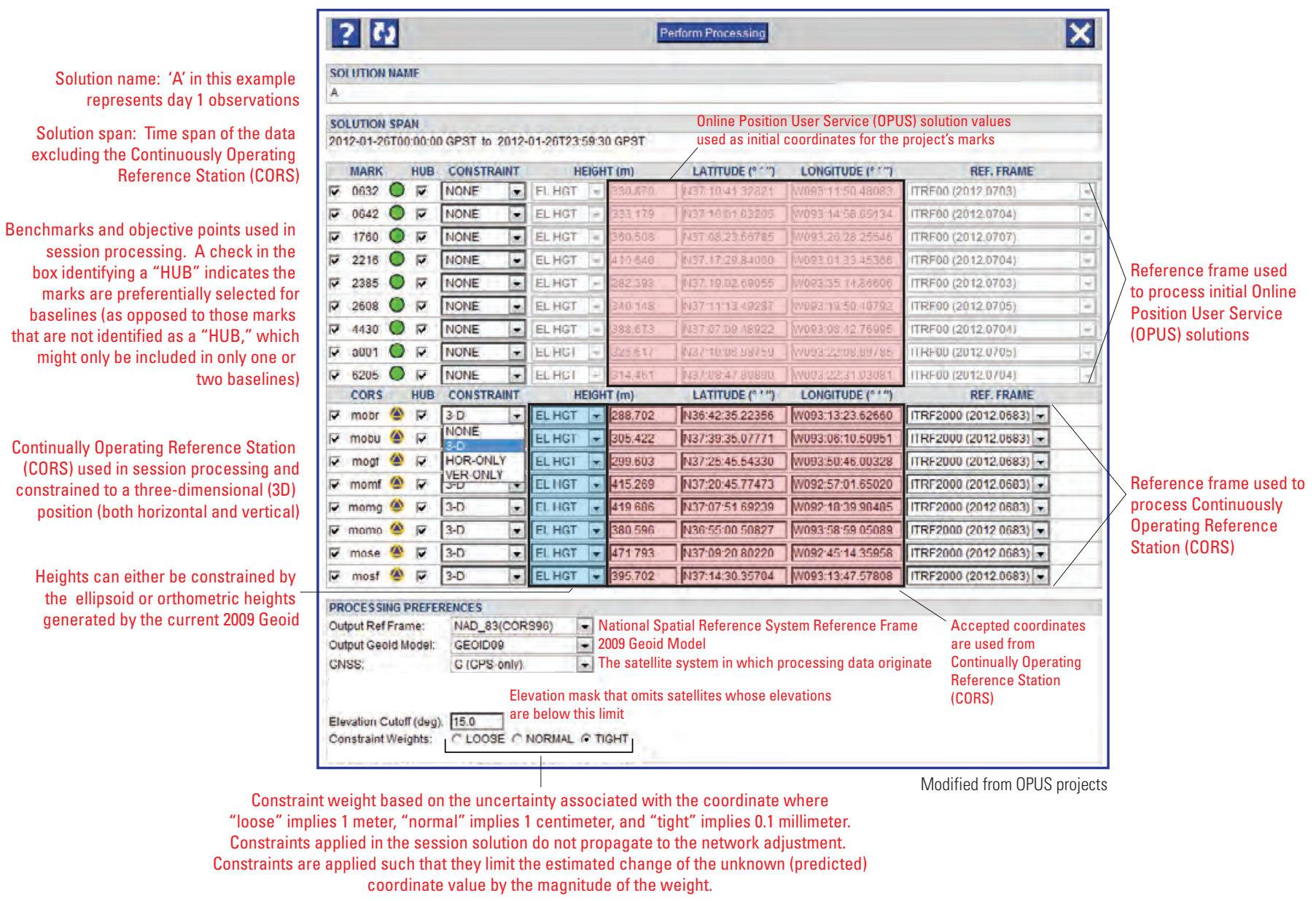

Figure 4-14. Online Position User Service (OPUS) projects session processing of a network of Global Navigation Satellite System (GNSS) observations.

Hubs were designated among all marks and CORS stations such that these would be preferentially selected for baseline development. All processing preferences, such as the output reference frame and geoid model, the elevation mask or cut off angle, and the weighted constraint criteria were selected to perform processing of a solution (fig. 4-14).

Once session solutions had been performed and optimized to produce the most effective session result, both sessions (day 1 and day 2) were combined into an adjustment. In this example, all benchmarks were constrained to the vertical only, ensuring the orthometric derived height (Geoid09) had been selected and the known elevations for each benchmark entered (fig. 4-15). Also, CORS were again (similar to session processing) constrained horizontally and vertically (3D) and to the orthometric height (Geoid09). Processing preferences are similar to session procession regarding the output reference frame, geoid model (2009), and constraint weights (fig. 4-15).

Session processing and adjustment results are available by email from OPUS. For this example, residuals of benchmarks $1760,2216,2385$, and 4430 that were used as constraints, were tabulated as well as a check on benchmark 'a001' (table 4-2). Residual values resulting from comparing known and final adjusted values were within $0.03 \mathrm{~m}$ in this example. Also, the occupation time among objective points was a minimum of 2.5 hours (as opposed to the 1-hour minimum requirement within the Level II criteria). Although the baseline lengths of the Level II criteria were exceeded when choosing to include additional exterior CORS, it would be feasible to represent the survey as Level II quality (despite baseline exceedence) based on additional occupation time for objective points, and the residuals reflected by the final adjustment results of both session solutions (table 4-2).

Greater detail regarding session processing, adjustments, and evaluation of results can be obtained from training specific to OPUS-Projects. As referred to in the "Single-Base: Online Positioning User Service (OPUS)" section, OPUS-Projects is currently (2012) in beta release and training is offered to ensure successful use of OPUS-Projects, as well as being authorized for project creation and management. 
Benchmarks and objective points used in the adjustment

Benchmarks constrained vertical only with entered known values from National Geodetic Survey datasheet

Continually Operating Reference Station (CORS) used in the adjustment and constrained to the orthometric height produced by the most current geoid (GEOID09)

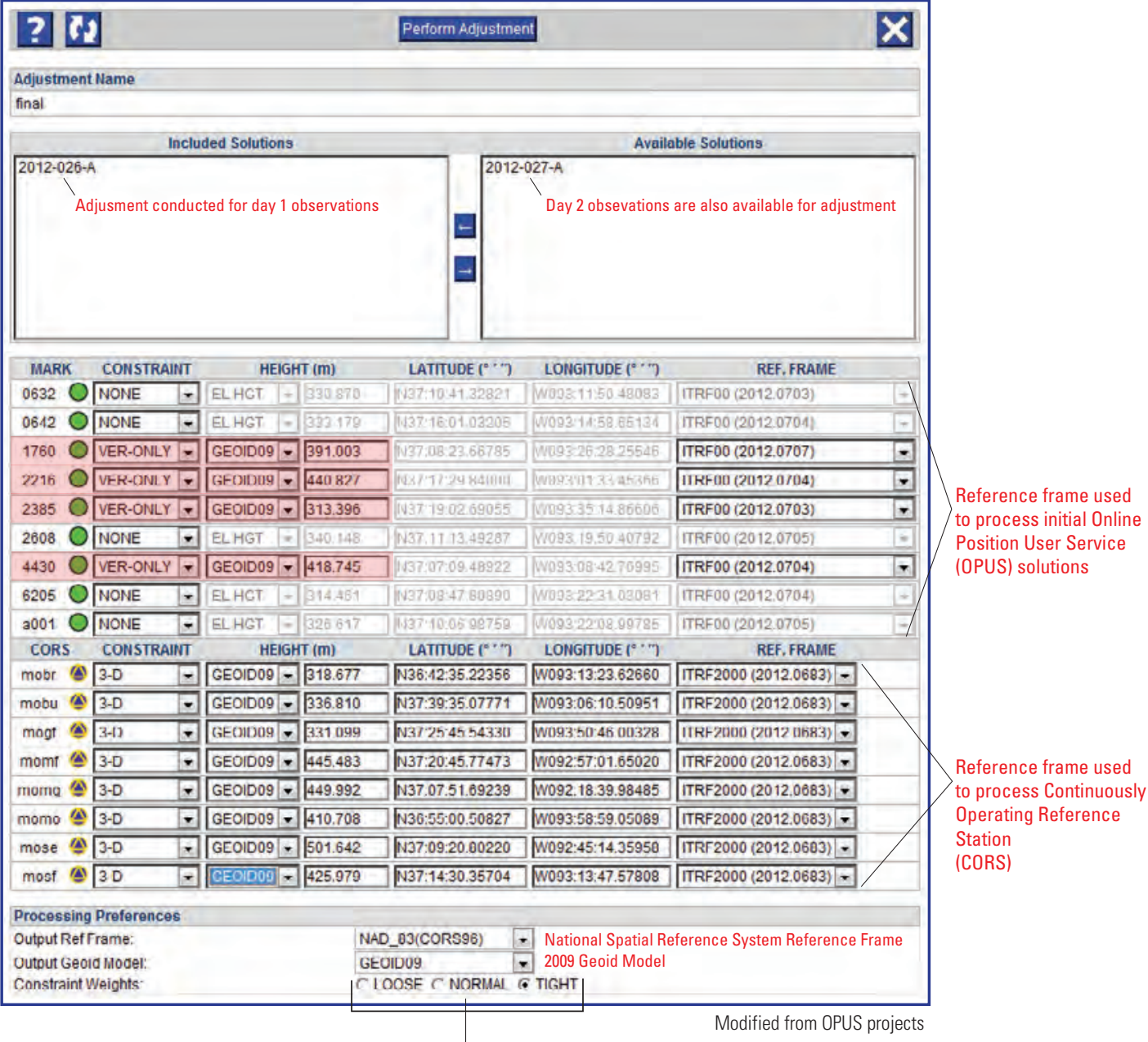

Constraint weight based on the uncertainty associated with the coordinate where

"loose" implies 1 meter, "normal" implies 1 centimeter, and "tight" implies 0.1 millimeter. Constraints applied in the session solution do not propagate to the network adjustment Constraints are applied such that they limit the estimated change of the unknown (predicted) coordinate value by the magnitude of the weight

Figure 4-15. Adjustment performed within the Online Position User Service (OPUS) Projects web-based utility.

Table 4-2. Static network survey uncertainty analysis using Online Position User Service (OPUS) projects results.

[m, meter; NAVD 88, North American Vertical Datum of 1988; --, no observation or no benchmark; NA, not applicable; red, vertically constrained marks during adjustment; a001, local city utility benchmark that was not constrained, but used as a check]

\begin{tabular}{rccccc}
\hline Mark & \multicolumn{2}{c}{ Processing session } & Adjustment & Benchmarks & Residual \\
\cline { 2 - 5 } & $\begin{array}{c}\text { Day 1 } \\
\text { elevation, } \\
\mathbf{m}\end{array}$ & $\begin{array}{c}\text { Day 2 } \\
\text { elevation, } \\
\mathbf{m}\end{array}$ & $\begin{array}{c}\text { Elevation, } \\
\mathbf{m}\end{array}$ & $\begin{array}{c}\text { 2d order NAVD 88 } \\
\text { elevation, } \\
\text { m }\end{array}$ & $\begin{array}{c}\text { ievation, } \\
\mathbf{m}\end{array}$ \\
\hline 0632 & 360.991 & 360.99 & 361.000 & -- & NA \\
0642 & 363.530 & 363.52 & 363.530 & -- & NA \\
1760 & 390.875 & 390.91 & 390.889 & 390.910 & -0.021 \\
2216 & 440.781 & 440.79 & 440.784 & 440.802 & -0.018 \\
2385 & 313.346 & 313.37 & 313.367 & 313.396 & -0.029 \\
2608 & 370.475 & -- & 370.494 & -- & NA \\
4430 & 418.712 & 418.73 & 418.726 & 418.745 & -0.019 \\
6205 & 344.791 & -- & 344.816 & -- & NA \\
a001 & -- & 373.26 & 373.264 & 373.266 & -0.002 \\
\hline
\end{tabular}



Publishing support provided by: Rolla Publishing Service Center

For more information concerning this publication, contact: Chief, USGS Office of Surface Water

12201 Sunrise Valley Drive

Reston, VA 20192

(703) 648-5301

Or visit the Office of Surface Water Web site at: http://water.usgs.gov/osw/ 
Back cover. Datum establishment for high-water mark surveys post Hurricane Irene at the Neuse River in New Bern, North Carolina. Photograph by Brian Pointer, 2011, USGS. 


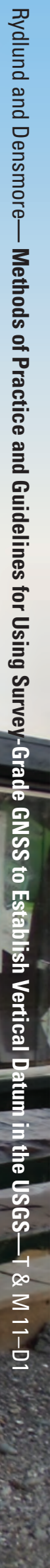

